Cochrane Database of Systematic Reviews

\title{
Non-pharmacological interventions for treating chronic prostatitis/ chronic pelvic pain syndrome (Review)
}

Franco JVA, Turk T, Jung JH, Xiao YT, lakhno S, Garrote V, Vietto V

Franco JVA, Turk T, Jung JH, Xiao YT, lakhno S, Garrote V, Vietto V.

Non-pharmacological interventions for treating chronic prostatitis/chronic pelvic pain syndrome.

Cochrane Database of Systematic Reviews 2018, Issue 5. Art. No.: CD012551.

DOI: 10.1002/14651858.CD012551.pub3.

www.cochranelibrary.com 
TABLE OF CONTENTS

HEADER

ABSTRACT

PLAIN LANGUAGE SUMMARY

SUMMARY OF FINDINGS

BACKGROUND

OBJECTIVES

METHODS

RESULTS

Figure 1.

Figure 2.

Figure 3.

Figure 4.

DISCUSSION

AUTHORS' CONCLUSIONS

ACKNOWLEDGEMENTS

REFERENCES

CHARACTERISTICS OF STUDIES

DATA AND ANALYSES

Analysis 1.1. Comparison 1 Acupuncture versus sham procedure, Outcome 1 Prostatitis symptoms (NIH-CPSI total).

Analysis 1.2. Comparison 1 Acupuncture versus sham procedure, Outcome 2 Prostatitis symptoms: pain subscore. .................

Analysis 1.3. Comparison 1 Acupuncture versus sham procedure, Outcome 3 Prostatitis symptoms: micturition subscore. .....

Analysis 1.4. Comparison 1 Acupuncture versus sham procedure, Outcome 4 Prostatitis symptoms: quality of life subscore. ....

Analysis 1.5. Comparison 1 Acupuncture versus sham procedure, Outcome 5 Prostatitis symptoms.

Analysis 1.6. Comparison 1 Acupuncture versus sham procedure, Outcome 6 Prostatitis symptoms (NIH-CPSI total) - medium term.

Analysis 1.7. Comparison 1 Acupuncture versus sham procedure, Outcome 7 Prostatitis symptoms: pain subscore - medium term.

Analysis 1.8. Comparison 1 Acupuncture versus sham procedure, Outcome 8 Prostatitis symptoms: micturition subscore medium term.

Analysis 1.9. Comparison 1 Acupuncture versus sham procedure, Outcome 9 Prostatitis symptoms: quality of life subscore medium term.

Analysis 1.10. Comparison 1 Acupuncture versus sham procedure, Outcome 10 Adverse events.

Analysis 1.11. Comparison 1 Acupuncture versus sham procedure, Outcome 11 Sexual dysfunction.

Analysis 1.12. Comparison 1 Acupuncture versus sham procedure, Outcome 12 Urinary symptoms.

Analysis 2.1. Comparison 2 Acupuncture treatments versus medical treatment, Outcome 1 Prostatitis symptoms (NIH-CPSI total).

Analysis 2.2. Comparison 2 Acupuncture treatments versus medical treatment, Outcome 2 Prostatitis symptoms: pain subscore.

Analysis 2.3. Comparison 2 Acupuncture treatments versus medical treatment, Outcome 3 Prostatitis symptoms: micturition subscore.

Analysis 2.4. Comparison 2 Acupuncture treatments versus medical treatment, Outcome 4 Prostatitis symptoms: quality of life subscore.

Analysis 2.5. Comparison 2 Acupuncture treatments versus medical treatment, Outcome 5 Prostatitis symptoms.

Analysis 2.6. Comparison 2 Acupuncture treatments versus medical treatment, Outcome 6 Adverse events.

Analysis 2.7. Comparison 2 Acupuncture treatments versus medical treatment, Outcome 7 Urinary symptoms.

Analysis 3.1. Comparison 3 Acupuncture with or without moxibustion, Outcome 1 Prostatitis symptoms (NIH-CPSI total). ......

Analysis 4.1. Comparison 4 Circumcision versus waiting list, Outcome 1 Prostatitis symptoms.

Analysis 4.2. Comparison 4 Circumcision versus waiting list, Outcome 2 Prostatitis symptoms: pain subscore. .......................

Analysis 4.3. Comparison 4 Circumcision versus waiting list, Outcome 3 Prostatitis symptoms: micturition subscore. ..............

Analysis 4.4. Comparison 4 Circumcision versus waiting list, Outcome 4 Prostatitis symptoms: quality of life subscore. ..........

Analysis 4.5. Comparison 4 Circumcision versus waiting list, Outcome 5 Adverse events.

Analysis 5.1. Comparison 5 Electromagnetic chair versus sham procedure, Outcome 1 Prostatitis symptoms (NIH-CPSI total). 
Analysis 5.2. Comparison 5 Electromagnetic chair versus sham procedure, Outcome 2 Prostatitis symptoms: pain subscore. ....

Analysis 5.3. Comparison 5 Electromagnetic chair versus sham procedure, Outcome 3 Prostatitis symptoms: micturition subscore.

Analysis 5.4. Comparison 5 Electromagnetic chair versus sham procedure, Outcome 4 Prostatitis symptoms: quality of life subscore.

Analysis 5.5. Comparison 5 Electromagnetic chair versus sham procedure, Outcome 5 Adverse events.

Analysis 5.6. Comparison 5 Electromagnetic chair versus sham procedure, Outcome 6 Urinary symptoms.

Analysis 5.7. Comparison 5 Electromagnetic chair versus sham procedure, Outcome 7 Prostatitis symptoms (NIH-CPSI total) medium term.

Analysis 5.8. Comparison 5 Electromagnetic chair versus sham procedure, Outcome 8 Prostatitis symptoms: pain subscore medium term.

Analysis 6.1. Comparison 6 Lifestyle modifications versus control, Outcome 1 Prostatitis symptoms.

Analysis 7.1. Comparison 7 Physical activity versus control, Outcome 1 Prostatitis symptoms.

Analysis 7.2. Comparison 7 Physical activity versus control, Outcome 2 Prostatitis symptoms: pain subscore. ......................... Analysis 7.3. Comparison 7 Physical activity versus control, Outcome 3 Prostatitis symptoms: micturition subscore. ............... Analysis 7.4. Comparison 7 Physical activity versus control, Outcome 4 Prostatitis symptoms: quality of life subscore. ............ Analysis 7.5. Comparison 7 Physical activity versus control, Outcome 5 Anxiety.

Analysis 7.6. Comparison 7 Physical activity versus control, Outcome 6 Depression.

Analysis 8.1. Comparison 8 Prostatic massage versus control, Outcome 1 Prostatitis symptoms.

Analysis 8.2. Comparison 8 Prostatic massage versus control, Outcome 2 Prostatitis symptoms: pain subscore. ...................... Analysis 8.3. Comparison 8 Prostatic massage versus control, Outcome 3 Prostatitis symptoms: micturition subscore. ........... Analysis 8.4. Comparison 8 Prostatic massage versus control, Outcome 4 Prostatitis symptoms: quality of life subscore. ......... Analysis 9.1. Comparison 9 Extracorporeal shockwave therapy (ESWT) versus control, Outcome 1 Prostatitis symptoms (NIHCPSI total).

Analysis 9.2. Comparison 9 Extracorporeal shockwave therapy (ESWT) versus control, Outcome 2 Prostatitis symptoms: pain subscore.

Analysis 9.3. Comparison 9 Extracorporeal shockwave therapy (ESWT) versus control, Outcome 3 Prostatitis symptoms: micturition subscore.

Analysis 9.4. Comparison 9 Extracorporeal shockwave therapy (ESWT) versus control, Outcome 4 Prostatitis symptoms: quality of life subscore.

Analysis 9.5. Comparison 9 Extracorporeal shockwave therapy (ESWT) versus control, Outcome 5 Prostatitis symptoms. ....... Analysis 9.6. Comparison 9 Extracorporeal shockwave therapy (ESWT) versus control, Outcome 6 Prostatitis symptoms (total score) - long term.

Analysis 9.7. Comparison 9 Extracorporeal shockwave therapy (ESWT) versus control, Outcome 7 Prostatitis symptoms: pain subscore - long term.

Analysis 9.8. Comparison 9 Extracorporeal shockwave therapy (ESWT) versus control, Outcome 8 Prostatitis symptoms: micturition subscore - long term.

Analysis 9.9. Comparison 9 Extracorporeal shockwave therapy (ESWT) versus control, Outcome 9 Prostatitis symptoms: quality of life subscore - long term.

Analysis 9.10. Comparison 9 Extracorporeal shockwave therapy (ESWT) versus control, Outcome 10 Adverse events. .............. Analysis 9.11. Comparison 9 Extracorporeal shockwave therapy (ESWT) versus control, Outcome 11 Sexual dysfunction. ....... Analysis 9.12. Comparison 9 Extracorporeal shockwave therapy (ESWT) versus control, Outcome 12 Urinary symptoms. ........ Analysis 10.1. Comparison 10 Transrectal thermotherapy (TRT) versus medical treatment, Outcome 1 Prostatitis symptoms (NIH-CPSI total).

Analysis 10.2. Comparison 10 Transrectal thermotherapy (TRT) versus medical treatment, Outcome 2 Prostatitis symptoms: pain subscore.

Analysis 10.3. Comparison 10 Transrectal thermotherapy (TRT) versus medical treatment, Outcome 3 Prostatitis symptoms: micturition subscore.

Analysis 10.4. Comparison 10 Transrectal thermotherapy (TRT) versus medical treatment, Outcome 4 Prostatitis symptoms: quality of life subscore.

Analysis 11.1. Comparison 11 Biofeedback with or without electrical stimulation versus usual care, Outcome 1 Prostatitis symptoms (NIH-CPSI total).

Analysis 11.2. Comparison 11 Biofeedback with or without electrical stimulation versus usual care, Outcome 2 Prostatitis symptoms: pain subscore. 
Analysis 11.3. Comparison 11 Biofeedback with or without electrical stimulation versus usual care, Outcome 3 Prostatitis symptoms: micturition subscore.

Analysis 11.4. Comparison 11 Biofeedback with or without electrical stimulation versus usual care, Outcome 4 Prostatitis symptoms: quality of life subscore.

Analysis 12.1. Comparison 12 External radiofrequency hyperthermia with or without terazosin, Outcome 1 Prostatitis symptoms.

Analysis 12.2. Comparison 12 External radiofrequency hyperthermia with or without terazosin, Outcome 2 Adverse events. ... Analysis 13.1. Comparison 13 Laser therapy versus medical treatment, Outcome 1 Prostatitis symptoms.

Analysis 13.2. Comparison 13 Laser therapy versus medical treatment, Outcome 2 Adverse events.

Analysis 14.1. Comparison 14 Tibial nerve stimulation versus no intervention, Outcome 1 Prostatitis symptoms (NIH-CPSI total).

Analysis 14.2. Comparison 14 Tibial nerve stimulation versus no intervention, Outcome 2 Prostatitis symptoms: pain subscore.

Analysis 14.3. Comparison 14 Tibial nerve stimulation versus no intervention, Outcome 3 Prostatitis symptoms: micturition subscore.

Analysis 14.4. Comparison 14 Tibial nerve stimulation versus no intervention, Outcome 4 Prostatitis symptoms: quality of life subscore.

Analysis 15.1. Comparison 15 Myofascial therapy versus control intervention, Outcome 1 Prostatitis symptoms (NIH-CPSI total).

Analysis 15.2. Comparison 15 Myofascial therapy versus control intervention, Outcome 2 Prostatitis symptoms: pain subscore.

Analysis 15.3. Comparison 15 Myofascial therapy versus control intervention, Outcome 3 Prostatitis symptoms: micturition subscore.

Analysis 15.4. Comparison 15 Myofascial therapy versus control intervention, Outcome 4 Prostatitis symptoms: quality of life subscore.

Analysis 15.5. Comparison 15 Myofascial therapy versus control intervention, Outcome 5 Sexual dysfunction.

Analysis 15.6. Comparison 15 Myofascial therapy versus control intervention, Outcome 6 Quality of life - physical.

Analysis 15.7. Comparison 15 Myofascial therapy versus control intervention, Outcome 7 Quality of life - mental.

Analysis 16.1. Comparison 16 Osteopathy versus sham procedure, Outcome 1 Prostatitis symptoms (NIH-CPSI total).

Analysis 16.2. Comparison 16 Osteopathy versus sham procedure, Outcome 2 Prostatitis symptoms: quality of life subscore. ..

Analysis 16.3. Comparison 16 Osteopathy versus sham procedure, Outcome 3 Urinary symptoms.

Analysis 17.1. Comparison 17 Sono-electromagnetic therapy versus placebo, Outcome 1 Prostatitis symptoms (NIH-CPSI total).

Analysis 17.2. Comparison 17 Sono-electromagnetic therapy versus placebo, Outcome 2 Prostatitis symptoms. .................... Analysis 17.3. Comparison 17 Sono-electromagnetic therapy versus placebo, Outcome 3 Prostatitis symptoms: pain subscore. Analysis 17.4. Comparison 17 Sono-electromagnetic therapy versus placebo, Outcome 4 Prostatitis symptoms: micturition subscore.

Analysis 17.5. Comparison 17 Sono-electromagnetic therapy versus placebo, Outcome 5 Prostatitis symptoms: quality of life subscore.

Analysis 18.1. Comparison 18 Transelectrical nerve stimulation (TENS) versus control, Outcome 1 Prostatitis symptoms: pain subscore.

Analysis 19.1. Comparison 19 Transurethral microwave thermotherapy, Outcome 1 Prostatitis symptoms (NIH-CPSI total). .... Analysis 19.2. Comparison 19 Transurethral microwave thermotherapy, Outcome 2 Prostatitis symptoms: pain subscore. ....... Analysis 19.3. Comparison 19 Transurethral microwave thermotherapy, Outcome 3 Prostatitis symptoms: micturition subscore.

Analysis 19.4. Comparison 19 Transurethral microwave thermotherapy, Outcome 4 Prostatitis symptoms: quality of life subscore.

Analysis 19.5. Comparison 19 Transurethral microwave thermotherapy, Outcome 5 Urinary symptoms.

Analysis 20.1. Comparison 20 Transurethral needle ablation (TUNA) versus sham procedure, Outcome 1 Prostatitis symptoms (NIH-CPSI total).

Analysis 20.2. Comparison 20 Transurethral needle ablation (TUNA) versus sham procedure, Outcome 2 Urinary symptoms. .. Analysis 21.1. Comparison 21 Ultrasound (non-intrusive), Outcome 1 Prostatitis symptoms (NIH-CPSI total).

Analysis 21.2. Comparison 21 Ultrasound (non-intrusive), Outcome 2 Prostatitis symptoms: pain subscore. 
Analysis 22.1. Comparison 22 Acupuncture treatments versus medical treatment - sensitivity analysis, Outcome 1 Prostatitis symptoms (NIH-CPSI total).

ADDITIONAL TABLES

APPENDICES

WHAT'S NEW

CONTRIBUTION

NS OF AUTHORS

DECLARATIONS OF INTEREST 
[Intervention Review]

\section{Non-pharmacological interventions for treating chronic prostatitis/ chronic pelvic pain syndrome}

Juan VA Franco1,2, Tarek Turk³, Jae Hung Jung ${ }^{4,5}$, Yu-Tian Xiao 6 , Stanislav lakhno7, Virginia Garrote8, Valeria Vietto ${ }^{1,2}$

1 Argentine Cochrane Centre, Instituto Universitario Hospital Italiano, Buenos Aires, Argentina. 2Family and Community Medicine Service, Hospital Italiano de Buenos Aires, Buenos Aires, Argentina. ${ }^{3}$ Faculty of Medicine, Damascus University, Damascus, Syrian Arab Republic. ${ }^{4}$ Department of Urology, Yonsei University Wonju College of Medicine, Wonju, Korea, South. ${ }^{5}$ Institute of Evidence Based Medicine, Yonsei University Wonju College of Medicine, Wonju, Korea, South. ${ }^{6}$ Department of Urology, Changhai Hospital, Second Military Medical

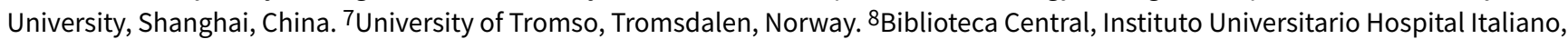
Buenos Aires, Argentina

Contact address: Juan VA Franco, Argentine Cochrane Centre, Instituto Universitario Hospital Italiano, Potosí 4234, Buenos Aires, Buenos Aires, C1199ACL, Argentina.juan.franco@hospitalitaliano.org.ar, jvaf85@gmail.com.

Editorial group: Cochrane Urology Group.

Publication status and date: Edited (no change to conclusions), published in Issue 5, 2018.

Citation: Franco JVA, Turk T, Jung JH, Xiao YT, lakhno S, Garrote V, Vietto V. Non-pharmacological interventions for treating chronic prostatitis/chronic pelvic pain syndrome. Cochrane Database of Systematic Reviews 2018, Issue 5. Art. No.: CD012551. DOI: 10.1002/14651858.CD012551.pub3.

Copyright @ 2018 The Cochrane Collaboration. Published by John Wiley \& Sons, Ltd.

\section{A B S T R A C T}

\section{Background}

Chronic prostatitis/chronic pelvic pain syndrome (CP/CPPS) is a common disorder in which the two main clinical features are pelvic pain and lower urinary tract symptoms. There are currently many approaches for its management, using both pharmacological and non-pharmacological interventions. The National Institute of Health - Chronic Prostatitis Symptom Index (NIH-CPSI) score is a validated measure commonly used to measure CP/CPPS symptoms.

\section{Objectives}

To assess the effects of non-pharmacological therapies for chronic prostatitis/chronic pelvic pain syndrome (CP/CPPS).

\section{Search methods}

We performed a comprehensive search using multiple databases, trial registries, grey literature and conference proceedings with no restrictions on the language of publication or publication status. The date of the latest search of all databases was August 2017.

\section{Selection criteria}

We included randomised controlled trials. Inclusion criteria were men with a diagnosis of CP/CPPS. We included all available nonpharmacological interventions.

\section{Data collection and analysis}

Two review authors independently classified studies and abstracted data from the included studies, performed statistical analyses and rated quality of evidence (QoE) according to the GRADE methods.

\section{Main results}

We included 38 unique studies with 3290 men with CP/CPPS across 23 comparisons. 
1. Acupuncture: (three studies, 204 participants) based on short-term follow-up, acupuncture probably leads to clinically meaningful reduction in prostatitis symptoms compared with sham procedure (mean difference (MD) in total NIH-CPSI score -5.79, $95 \%$ confidence interval $(\mathrm{Cl})-7.32$ to -4.26 , high QoE). Acupuncture may result in little to no difference in adverse events (low QoE). Acupuncture may not reduce sexual dysfunction when compared with sham procedure (MD in the International Index of Erectile Function (IIEF) Scale -0.50, 95\% $\mathrm{Cl}-3.46$ to 2.46, low $\mathrm{QoE}$ ). Acupuncture may also lead to a clinically meaningful reduction in prostatitis symptoms compared with standard medical therapy (MD $-6.05,95 \% \mathrm{Cl}-7.87$ to -4.24 , two studies, 78 participants, low QoE). We found no information regarding quality of life, depression or anxiety.

2. Lifestyle modifications: (one study, 100 participants) based on short-term follow-up, lifestyle modifications may be associated with a reduction in prostatitis symptoms compared with control (risk ratio (RR) for improvement in $\mathrm{NIH}-\mathrm{CPSI}$ scores $3.90,95 \% \mathrm{Cl} 2.20$ to 6.92 , very low QoE). We found no information regarding adverse events, sexual dysfunction, quality of life, depression or anxiety.

3. Physical activity: (one study, 85 participants) based on short-term follow-up, a physical activity programme may cause a small reduction in prostatitis symptoms compared with control (NIH-CPSI score MD $-2.50,95 \% \mathrm{Cl}-4.69$ to -0.31 , low QoE). This programme may not reduce anxiety or depression (low QoE). We found no information regarding adverse events, sexual dysfunction or quality of life.

4. Prostatic massage: (two studies, 115 participants) based on short-term follow-up, we are uncertain whether the prostatic massage reduces or increases prostatitis symptoms compared with control (very low QoE). We found no information regarding adverse events, sexual dysfunction, quality of life, depression or anxiety.

5. Extracorporeal shockwave therapy: (three studies, 157 participants) based on short-term follow-up, extracorporeal shockwave therapy reduces prostatitis symptoms compared with control (NIH-CPSI score MD $-6.18,95 \% \mathrm{Cl}-7.46$ to -4.89 , high QoE). These results may not be sustained at medium-term follow-up (low QoE). This treatment may not be associated with a greater incidence of adverse events (low $\mathrm{QOE}$ ). This treatment probably improves sexual dysfunction (MD in the IIEF Scale MD $3.34,95 \% \mathrm{Cl} 2.68$ to 4.00 , one study, 60 participants, moderate QoE). We found no information regarding quality of life, depression or anxiety.

6. Transrectal thermotherapy compared to medical therapy: (two studies, 237 participants) based on short-term follow-up, transrectal thermotherapy alone or in combination with medical therapy may decrease prostatitis symptoms slightly when compared with medical therapy alone (NIH-CPSI score MD $-2.50,95 \% \mathrm{Cl}-3.82$ to -1.18 , low QoE). One included study reported that participants may experience transient adverse events. We found no information regarding sexual dysfunction, quality of life, depression or anxiety.

7. Other interventions: there is uncertainty about the effects of most of the other interventions included in this review. We found no information regarding psychological support or prostatic surgery.

\section{Authors' conclusions}

Based on the findings of moderate quality evidence, this review found that some non-pharmacological interventions such as acupuncture and extracorporeal shockwave therapy are likely to result in a decrease in prostatitis symptoms and may not be associated with a greater incidence of adverse event. The QoE for most other comparisons was predominantly low. Future clinical trials should include a full report of their methods including adequate masking, consistent assessment of all patient-important outcomes including potential treatmentrelated adverse events and appropriate sample sizes.

\section{PLAIN LANGUAGE SUMMARY}

\section{Intervention for treating chronic prostatitis and chronic pelvic pain in men}

\section{Review question}

What are the effects of non-medicine therapies in men with longstanding pain and discomfort around their prostate and pelvis, so-called chronic prostatitis/chronic pelvic pain syndrome (CP/CPPS)?

\section{Background}

CP/CPPS is a common disorder in which men feel pelvic pain or have bothersome symptoms (or both of these) when urinating. Its cause is unknown and there are many different treatments for this condition.

\section{Study characteristics}

The evidence was current to August 2017. We found 38 studies that were conducted between 1993 and 2016 with 3187 participants that made 23 comparisons between different treatments in men with CP/CPPS. The evaluated interventions usually implied the use of devices, medical advice or some form of physical therapy. In many cases, these therapies were given to men in an outpatient setting. Most studies did not specify their funding sources; three studies reported funding from device makers.

\section{Key results}


Acupuncture: we found that acupuncture (an alternative medicine where thin needles are inserted into the skin at specific points) probably causes a significant decrease in symptoms of prostatitis and may not associated with side effects when compared with pretend acupuncture, however, it may not reduce sexual problems. It probably decreases symptoms when compared with standard medical therapy. We found no information on its effect on quality of life, depression or anxiety.

Lifestyle modifications: we are uncertain whether the recommendation of lifestyle modifications reduces symptoms when compared to the continuation of the same lifestyle. We had no information regarding side effects, sexual problems, quality of life, depression or anxiety.

Physical activity: we found that a physical activity programme may reduce symptoms (small effect) when compared with a non-specific activity used as a control, however it may not reduce anxiety or depression. We have no information regarding side effects, sexual problems or quality of life.

Prostatic massage: we are uncertain whether the prostatic massage reduces or increases symptoms when compared with no massage. We found no information regarding side effects, sexual problems, quality of life, depression or anxiety.

Extracorporeal shockwave therapy: we found that extracorporeal shockwave therapy (where shock waves are passed through the skin to the prostate) causes a significant decrease in symptoms compared to a simulated procedure. These results may not be lasting after more continued treatment. This treatment may not be associated with side effects. We have no information regarding quality of life, depression or anxiety.

Transrectal thermotherapy compared to medical therapy: we found that transrectal thermotherapy (which applies heat to the prostate and pelvic muscle area) alone or in combination with medical therapy may cause a small decrease in symptoms compared to medical therapy alone. One of the included studies reported that participants may experience transient side effects. We have no information regarding sexual problems, quality of life, depression or anxiety.

There is uncertainty about the effects of other interventions.

\section{Quality of the evidence}

The quality of the evidence was low in most cases, meaning that there is much uncertainty surrounding the results. The included studies were not well designed, had a small sample size and had a short follow-up time (usually 12 weeks). 


\begin{tabular}{|c|c|c|c|c|c|c|}
\hline \multirow{13}{*}{ 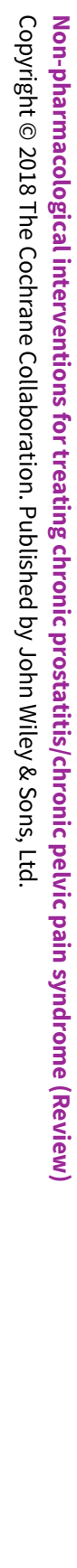 } & \multicolumn{6}{|c|}{$\begin{array}{l}\text { S U M M A R Y O F F I N D I N G S } \\
\text { Summary of findings for the main comparison. Acupunc } \\
\text { syndrome }\end{array}$} \\
\hline & \multicolumn{6}{|c|}{ Acupuncture compared to sham procedure for chronic prostatitis/chronic pelvic pain syndrome } \\
\hline & \multicolumn{6}{|c|}{$\begin{array}{l}\text { Patient or population: participants with chronic prostatitis/chronic pelvic pain syndrome } \\
\text { Setting: outpatient, Korea, Malaysia, US and Turkey } \\
\text { Intervention: acupuncture } \\
\text { Comparison: sham procedure placing needles in different points from those used in acupuncture }\end{array}$} \\
\hline & \multirow[t]{2}{*}{ Outcomes } & \multirow{2}{*}{$\begin{array}{l}\text { № of participants } \\
\text { (studies) }\end{array}$} & \multirow{2}{*}{$\begin{array}{l}\text { Quality of the evi- } \\
\text { dence } \\
\text { (GRADE) }\end{array}$} & \multirow{2}{*}{$\begin{array}{l}\text { Relative effect } \\
(95 \% \mathrm{CI})\end{array}$} & \multicolumn{2}{|c|}{ Anticipated absolute effects ${ }^{\star}(95 \% \mathrm{Cl})$} \\
\hline & & & & & $\begin{array}{l}\text { Risk with sham pro- } \\
\text { cedure }\end{array}$ & $\begin{array}{l}\text { Risk difference with } \\
\text { Acupuncture }\end{array}$ \\
\hline & $\begin{array}{l}\text { Prostatitis Symptoms } \\
\text { assessed with: NIH-CPSI score } \\
\text { Scale from: } 0 \text { to } 43 \\
\text { follow up: } 6-8 \text { weeks } \\
\text { Benefit is indicated by lower scores }\end{array}$ & $\begin{array}{l}204 \\
\text { (3 RCTs) }\end{array}$ & $\begin{array}{l}\oplus \oplus \oplus \odot \\
\text { Moderate } 1\end{array}$ & - & $\begin{array}{l}\text { The mean prostati- } \\
\text { tis Symptoms ranged } \\
\text { from } 17.08 \text { to } 22\end{array}$ & $\begin{array}{l}\text { MD } 5.79 \text { lower } \\
\text { (7.32 lower to } 4.26 \text { lower) }\end{array}$ \\
\hline & \multirow{2}{*}{$\begin{array}{l}\text { Prostatitis Symptoms } \\
\text { assessed with: NIH-CPSI score (response) } \\
\text { follow up: } 6 \text { weeks }\end{array}$} & \multirow{2}{*}{$\begin{array}{l}113 \\
\text { (2 RCTs) }\end{array}$} & \multirow{2}{*}{$\begin{array}{l}\oplus \ominus \ominus \ominus \\
\text { Very low } 123\end{array}$} & \multirow{2}{*}{$\begin{array}{l}\text { RR } 2.49 \\
(0.77 \text { to } 8.02)\end{array}$} & Study population & \\
\hline & & & & & 404 per 1.000 & $\begin{array}{l}\mathbf{5 9 6} \text { more per } \mathbf{1 0 0 0} \\
\text { (93 fewer to } 596 \text { more) }\end{array}$ \\
\hline & \multirow{2}{*}{$\begin{array}{l}\text { Adverse events } \\
\text { follow up: 6-8 weeks }\end{array}$} & \multirow{2}{*}{$\begin{array}{l}204 \\
\text { (3 RCTs) }\end{array}$} & \multirow{2}{*}{$\begin{array}{l}\oplus \oplus \ominus \ominus \\
\text { Low } 13\end{array}$} & \multirow{2}{*}{$\begin{array}{l}\text { RR } 1.33 \\
(0.51 \text { to } 3.46)\end{array}$} & \multicolumn{2}{|l|}{ Study population } \\
\hline & & & & & 58 per 1.000 & $\begin{array}{l}19 \text { more per } 1000 \\
\text { (29 fewer to } 143 \text { more) }\end{array}$ \\
\hline & $\begin{array}{l}\text { Sexual dysfunction } \\
\text { assessed with: International Index of Erectile } \\
\text { Function } \\
\text { Scale from: } 5 \text { to } 25 \\
\text { follow up: } 6 \text { weeks }\end{array}$ & $\begin{array}{l}89 \\
(1 \mathrm{RCT})\end{array}$ & $\begin{array}{l}\oplus \oplus \Theta \Theta \\
\text { Low } 14\end{array}$ & - & $\begin{array}{l}\text { The mean sexual } \\
\text { dysfunction was } 23\end{array}$ & $\begin{array}{l}\text { MD } 0.5 \text { lower } \\
\text { ( } 3.46 \text { lower to } 2.46 \text { higher) }\end{array}$ \\
\hline & \multicolumn{6}{|l|}{ Benefit is indicated by higher scores } \\
\hline & Quality of life - not reported & - & - & - & - & - \\
\hline
\end{tabular}


${ }^{\star}$ The risk in the intervention group (and its $95 \%$ confidence interval) is based on the assumed risk in the comparison group and the relative effect of the intervention (and its $95 \% \mathrm{Cl}$ ).

Cl: Confidence interval; RR: Risk ratio; OR: Odds ratio;

\section{GRADE Working Group grades of evidence}

High quality: We are very confident that the true effect lies close to that of the estimate of the effect

Moderate quality: We are moderately confident in the effect estimate: The true effect is likely to be close to the estimate of the effect, but there is a possibility that it is substantially different

Low quality: Our confidence in the effect estimate is limited: The true effect may be substantially different from the estimate of the effect

Very low quality: We have very little confidence in the effect estimate: The true effect is likely to be substantially different from the estimate of effect

1 Downgraded 1 level due to unclear risk of bias: insufficient information about allocation concealment

2 Downgraded 1 level due to inconsistency: statistical heterogeneity $(12=76 \%)$

3 Downgraded 1 level due to imprecision issues: wide confidence interval due to small sample size and few events.

4 Downgraded 1 level for imprecision issues: wide confidence interval includes both appreciable benefit and harm.

\section{Summary of findings 2. Acupuncture compared to medical treatment for treating chronic prostatitis/chronic pelvic pain syndrome}

\section{Acupuncture compared to medical treatment for chronic prostatitis/chronic pelvic pain syndrome}

Patient or population: treating chronic prostatitis/chronic pelvic pain syndrome

Setting: outpatient, Korea, China and Turkey

Intervention: acupuncture

Comparison: medical treatment

\begin{tabular}{|c|c|c|c|c|c|}
\hline \multirow[t]{2}{*}{ Outcomes } & \multirow{2}{*}{$\begin{array}{l}\text { № of participants } \\
\text { (studies) }\end{array}$} & \multirow{2}{*}{$\begin{array}{l}\text { Quality of the evi- } \\
\text { dence } \\
\text { (GRADE) }\end{array}$} & \multirow{2}{*}{$\begin{array}{l}\text { Relative effect } \\
(95 \% \mathrm{Cl})\end{array}$} & \multicolumn{2}{|c|}{ Anticipated absolute effects ${ }^{\star}(95 \% \mathrm{Cl})$} \\
\hline & & & & $\begin{array}{l}\text { Risk with medical } \\
\text { treatment }\end{array}$ & $\begin{array}{l}\text { Risk difference with } \\
\text { acupuncture }\end{array}$ \\
\hline $\begin{array}{l}\text { Prostatitis symptoms (NIH-CPSI total) } \\
\text { assessed with: NIH-CPSI score } \\
\text { Scale from: } 0 \text { to } 43 \\
\text { follow-up: } 12 \text { weeks }\end{array}$ & $\begin{array}{l}78 \\
(2 \mathrm{RCTs})\end{array}$ & $\begin{array}{l}\oplus \oplus \oplus \odot \\
\text { Moderate }\end{array}$ & - & $\begin{array}{l}\text { The mean prostatitis } \\
\text { symptoms (NIH-CPSI } \\
\text { total) ranged from } 12 \\
\text { to } 16\end{array}$ & $\begin{array}{l}\text { MD } 6.05 \text { lower } \\
\text { ( } 7.87 \text { lower to } 4.24 \\
\text { lower) }\end{array}$ \\
\hline \multicolumn{6}{|l|}{ Benefit is indicated by lower scores } \\
\hline \multirow{2}{*}{$\begin{array}{l}\text { Prostatitis symptoms: response defined as a 6- } \\
\text { point decrease in NIH-CPSI score } \\
\text { follow-up: } 6 \text { weeks } 3\end{array}$} & \multirow{2}{*}{$\begin{array}{l}24 \\
(1 \mathrm{RCT})\end{array}$} & \multirow{2}{*}{$\begin{array}{l}\oplus \oplus \oplus \ominus \\
\text { Low }^{1,3}\end{array}$} & \multirow{2}{*}{$\begin{array}{l}\text { RR } 3.57 \\
(1.45 \text { to } 8.80)\end{array}$} & \multicolumn{2}{|l|}{ Study population } \\
\hline & & & & 250 per 1000 & 643 more per 1000 \\
\hline
\end{tabular}




\begin{tabular}{|c|c|c|c|c|}
\hline & & & & $\begin{array}{l}\text { (112 more to } 1950 \\
\text { more) }\end{array}$ \\
\hline $\begin{array}{l}\text { Adverse events } \\
\text { follow-up: } 12 \text { weeks }\end{array}$ & $\begin{array}{l}78 \\
\text { (2 RCTs) }\end{array}$ & $\begin{array}{l}\oplus \oplus \oplus \ominus \\
\text { Low }^{1,3}\end{array}$ & - & There were no adverse events in either group. \\
\hline Sexual dysfunction - not reported & - & - & - & - \\
\hline Quality of life - not reported & - & - & - & - \\
\hline Depression and anxiety - not reported & - & - & - & - \\
\hline
\end{tabular}

${ }^{*}$ The risk in the intervention group (and its 95\% confidence interval) is based on the assumed risk in the comparison group and the relative effect of the intervention (and its $95 \% \mathrm{Cl}$ ).

CI: confidence interval; MD: mean difference; NIH-CPSI: National Institutes of Health - Chronic Prostatitis Symptom Index; RCT: randomised controlled trial; RR: risk ratio.

\section{GRADE Working Group grades of evidence}

High quality: we are very confident that the true effect lies close to that of the estimate of the effect.

Moderate quality: we are moderately confident in the effect estimate: the true effect is likely to be close to the estimate of the effect, but there is a possibility that it is substantially different.

Low quality: our confidence in the effect estimate is limited: the true effect may be substantially different from the estimate of the effect.

Very low quality: we have very little confidence in the effect estimate: the true effect is likely to be substantially different from the estimate of effect.

1Downgraded 1 level due to risk of bias: included studies were not blinded, which affects both detection and performance bias.

${ }^{2}$ The initial analysis had greater statistical inconsistency $(12=70 \%)$ and included one study that included people with chronic prostatitis/chronic pelvic pain syndrome using criteria that differed from that recommended by the Research Consensus (Chen 2009). In a sensitivity analysis, we excluded the results from this study and found greater statistical consistency $\left(I^{2}=0 \%\right)$, therefore, we chose to report these results in the 'Summary of findings' table. For this reason, we did not downgrade due to inconsistency.

${ }^{3}$ Downgraded 1 level due to imprecision issues: few events and wide confidence interval.

\section{Summary of findings 3. Circumcision plus usual care compared to waiting list plus usual care for chronic prostatitis/chronic pelvic pain syndrome}

Circumcision plus usual care compared to waiting list plus usual care for chronic prostatitis/chronic pelvic pain syndrome

Patient or population: participants with chronic prostatitis/chronic pelvic pain syndrome

Setting: hospital (surgery), China

Intervention: circumcision at 4 weeks (outcome was assessed after circumcision)

Comparison: waiting list for circumcision at 3 months (outcome was assessed before circumcision)

Outcomes

(studies)
Quality of the evi-

dence

(GRADE)
Relative effect

$(95 \% \mathrm{Cl})$
Anticipated absolute effects ${ }^{\star}(95 \% \mathrm{CI})$ 


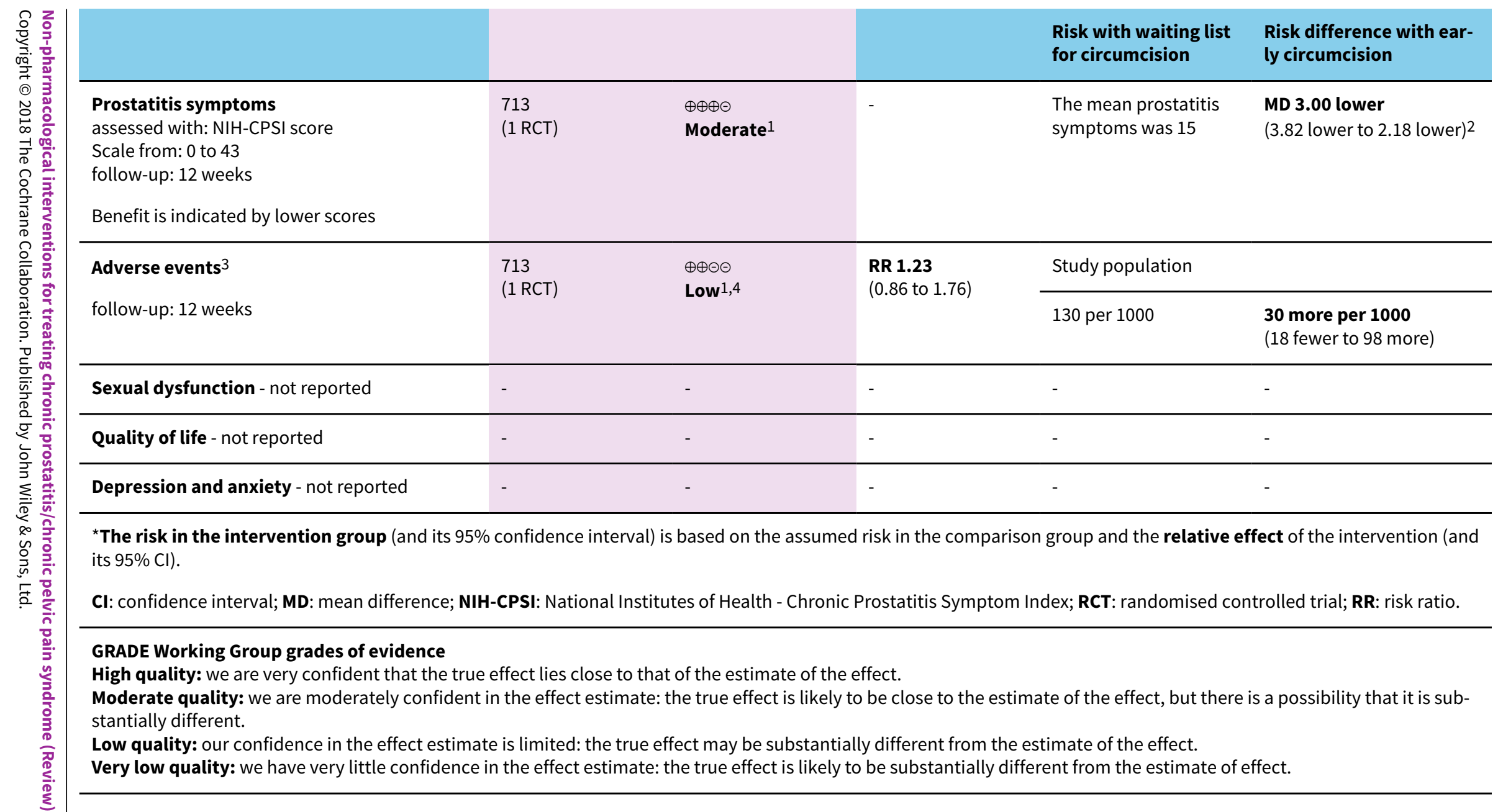

1Downgraded 1 level due to high risk of bias: study not blinded (high risk of performance and detection bias).

2Confidence intervals were constructed using transformations described in the Cochrane Handbook for Systematic Reviews of Interventions Section 7.7.3.5.

${ }^{3}$ All adverse events were minor.

4Downgraded 1 level due to imprecision issues: few events in each group and wide confidence interval.

\section{Summary of findings 4. Electromagnetic chair compared to control intervention for chronic prostatitis/chronic pelvic pain syndrome}

Electromagnetic chair compared to control intervention for chronic prostatitis/chronic pelvic pain syndrome 


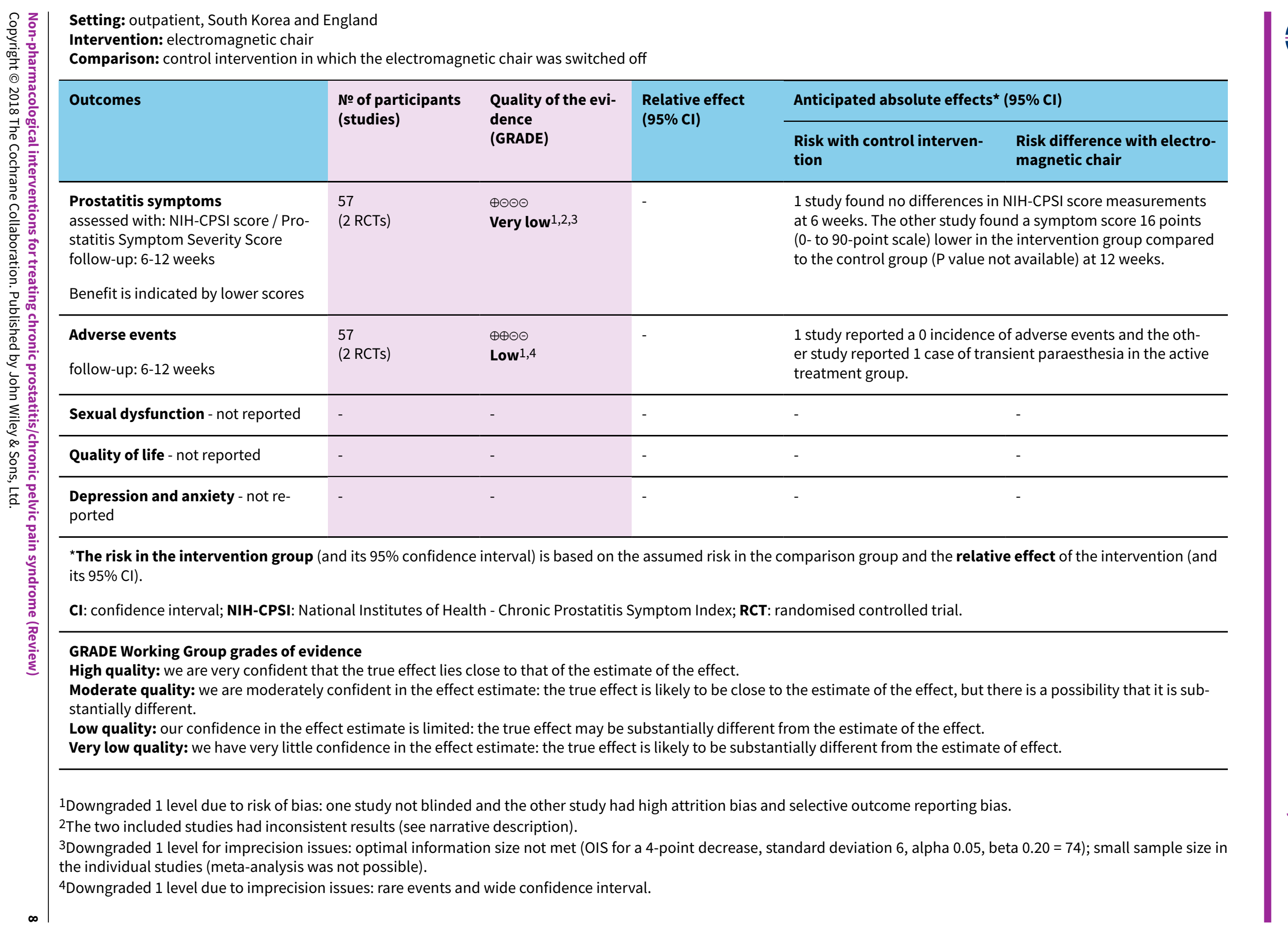




\section{Lifestyle modifications compared to control for chronic prostatitis/chronic pelvic pain syndrome}

Patient or population: participants with chronic prostatitis/chronic pelvic pain syndrome

Setting: outpatient, Italy

Intervention: lifestyle modifications based on the assessment of risk factors for chronic prostatitis/chronic pelvic pain syndrome

Comparison: control intervention in which participants were instructed to continue with the same lifestyle

\begin{tabular}{|c|c|c|c|c|c|}
\hline \multirow[t]{2}{*}{ Outcomes } & \multirow{2}{*}{$\begin{array}{l}\text { № of participants } \\
\text { (studies) } \\
\text { Follow-up }\end{array}$} & \multirow{2}{*}{$\begin{array}{l}\text { Quality of the evi- } \\
\text { dence } \\
\text { (GRADE) }\end{array}$} & \multirow{2}{*}{$\begin{array}{l}\text { Relative effect } \\
(95 \% \mathrm{Cl})\end{array}$} & \multicolumn{2}{|c|}{ Anticipated absolute effects ${ }^{\star}(95 \% \mathrm{Cl})$} \\
\hline & & & & Risk with control & $\begin{array}{l}\text { Risk difference with } \\
\text { lifestyle modifications }\end{array}$ \\
\hline \multirow{2}{*}{$\begin{array}{l}\text { Prostatitis symptoms: response defined as 6-point } \\
\text { decrease in NIH-CPSI score } \\
\text { follow-up: } 3 \text { months }\end{array}$} & \multirow{2}{*}{$\begin{array}{l}100 \\
(1 \mathrm{RCT})\end{array}$} & \multirow{2}{*}{$\begin{array}{l}\oplus \ominus \odot \ominus \\
\text { Very low } 1,2\end{array}$} & \multirow{2}{*}{$\begin{array}{l}\text { RR } 3.90 \\
\text { (2.20 to } 6.92)\end{array}$} & \multicolumn{2}{|l|}{ Study population } \\
\hline & & & & 200 per 1000 & $\begin{array}{l}\mathbf{5 8 0} \text { more per } 1000 \\
\text { ( } 240 \text { more to } 1184 \text { more) }\end{array}$ \\
\hline Adverse events - not reported & - & - & - & - & - \\
\hline Sexual dysfunction - not reported & - & - & - & - & - \\
\hline Quality of life - not reported & - & - & - & - & - \\
\hline Depression and anxiety - not reported & - & - & - & - & - \\
\hline
\end{tabular}

*The risk in the intervention group (and its 95\% confidence interval) is based on the assumed risk in the comparison group and the relative effect of the intervention (and its $95 \% \mathrm{Cl}$ ).

Cl: confidence interval; NIH-CPSI: National Institutes of Health - Chronic Prostatitis Symptom Index; RCT: randomised controlled trial; RR: risk ratio.

\section{GRADE Working Group grades of evidence}

High quality: we are very confident that the true effect lies close to that of the estimate of the effect.

Moderate quality: we are moderately confident in the effect estimate: the true effect is likely to be close to the estimate of the effect, but there is a possibility that it is substantially different.

Low quality: our confidence in the effect estimate is limited: the true effect may be substantially different from the estimate of the effect.

Very low quality: we have very little confidence in the effect estimate: the true effect is likely to be substantially different from the estimate of effect.

1Downgraded 2 levels due to high risk of selection bias (unconcealed allocation), detection and performance bias (study not blinded), missing outcome data and suspected selective outcome reporting (data presented graphically).

2Downgraded 1 level due to imprecision issues: few events and wide confidence interval. 
Physical activity compared to control intervention procedure for chronic prostatitis/chronic pelvic pain syndrome

Patient or population: participants with chronic prostatitis/chronic pelvic pain syndrome

Setting: outpatient, Italy

Intervention: physical activity programme

Comparison: control intervention comprising a flexibility and motion low-grade exercise programme

\begin{tabular}{|c|c|c|c|c|c|}
\hline \multirow[t]{2}{*}{ Outcomes } & \multirow{2}{*}{$\begin{array}{l}\text { № of participants } \\
\text { (studies) }\end{array}$} & \multirow{2}{*}{$\begin{array}{l}\text { Quality of the evi- } \\
\text { dence } \\
\text { (GRADE) }\end{array}$} & \multirow{2}{*}{$\begin{array}{l}\text { Relative effect } \\
(95 \% \mathrm{Cl})\end{array}$} & \multicolumn{2}{|c|}{ Anticipated absolute effects* $(95 \% \mathrm{Cl})$} \\
\hline & & & & $\begin{array}{l}\text { Risk with sham pro- } \\
\text { cedure }\end{array}$ & $\begin{array}{l}\text { Risk difference with } \\
\text { physical activity }\end{array}$ \\
\hline $\begin{array}{l}\text { Prostatitis symptoms } \\
\text { assessed with: NIH-CPSI score } \\
\text { Scale from: } 0 \text { to } 43 \\
\text { follow-up: } 6 \text { weeks }\end{array}$ & $\begin{array}{l}85 \\
(1 \mathrm{RCT})\end{array}$ & $\begin{array}{l}\oplus \oplus \odot \odot \\
\text { Low }^{1}\end{array}$ & - & $\begin{array}{l}\text { The mean prostatitis } \\
\text { symptom score was } \\
20\end{array}$ & $\begin{array}{l}\text { MD } 2.50 \text { lower } \\
\text { (4.69 lower to } 0.31 \text { low- } \\
\text { er) }\end{array}$ \\
\hline \multicolumn{6}{|l|}{ Benefit is indicated by lower scores } \\
\hline Adverse events - not reported & - & - & - & - & - \\
\hline Sexual dysfunction - not reported & - & - & - & - & - \\
\hline Quality of life - not reported & - & - & - & - & - \\
\hline $\begin{array}{l}\text { Anxiety } \\
\text { assessed with: SAI-Y score } \\
\text { Scale from: } 20 \text { to } 80 \\
\text { follow-up: } 6 \text { weeks }\end{array}$ & $\begin{array}{l}85 \\
(1 \mathrm{RCT})\end{array}$ & $\begin{array}{l}\oplus \ominus \ominus \ominus \\
\text { Very low } 1,2\end{array}$ & - & $\begin{array}{l}\text { The mean anxiety } \\
\text { score was } 42.1\end{array}$ & $\begin{array}{l}\text { MD } 2.8 \text { lower } \\
\text { ( } 6.78 \text { lower to } 1.18 \text { high- } \\
\text { er) }\end{array}$ \\
\hline \multicolumn{6}{|l|}{ Benefit is indicated by lower scores } \\
\hline $\begin{array}{l}\text { Depression } \\
\text { assessed with: Beck Depression Inventory } \\
\text { Scale from: } 0 \text { to } 63 \\
\text { follow-up: } 6 \text { weeks }\end{array}$ & $\begin{array}{l}85 \\
(1 \mathrm{RCT})\end{array}$ & $\begin{array}{l}\oplus \ominus \ominus \ominus \\
\text { Very low } 1,2\end{array}$ & - & $\begin{array}{l}\text { The mean depres- } \\
\text { sion score was } 9.3\end{array}$ & $\begin{array}{l}\text { MD } \mathbf{0 . 5} \text { higher } \\
\text { (1.33 lower to } 2.33 \text { high- } \\
\text { er) }\end{array}$ \\
\hline Benefit is indicated by lower scores & & & & & \\
\hline
\end{tabular}

${ }^{*}$ The risk in the intervention group (and its 95\% confidence interval) is based on the assumed risk in the comparison group and the relative effect of the intervention (and its $95 \% \mathrm{Cl}$ ). 


\begin{tabular}{|c|c|c|c|c|c|}
\hline \multicolumn{6}{|c|}{$\begin{array}{l}\text { CI: confidence interval; MD: mean difference; NIH-CPSI: National Institutes of Health - Chronic Prostatitis Symptom Index; RCT: randomised controlled trial; SAI-Y: State } \\
\text { Anxiety Inventory-Y. }\end{array}$} \\
\hline \multicolumn{6}{|c|}{$\begin{array}{l}\text { GRADE Working Group grades of evidence } \\
\text { High quality: we are very confident that the true effect lies close to that of the estimate of the effect. } \\
\text { Moderate quality: we are moderately confident in the effect estimate: the true effect is likely to be close to the estimate of the effect, but there is a possibility that it is sub- } \\
\text { stantially different. } \\
\text { Low quality: our confidence in the effect estimate is limited: the true effect may be substantially different from the estimate of the effect. } \\
\text { Very low quality: we have very little confidence in the effect estimate: the true effect is likely to be substantially different from the estimate of effect. }\end{array}$} \\
\hline \multicolumn{6}{|c|}{$\begin{array}{l}\text { 1Downgraded } 2 \text { levels: high risk of performance bias and detection bias (study not blinded); high risk of attrition bias at follow-up. } \\
\text { 2Downgraded } 1 \text { level due to imprecision issues: wide confidence intervals include both considerable benefits and harms. }\end{array}$} \\
\hline \multicolumn{6}{|c|}{ Prostatic massage compared to control for treating chronic prostatitis/chronic pelvic pain syndrome } \\
\hline \multicolumn{6}{|c|}{$\begin{array}{l}\text { Patient or population: participants with chronic prostatitis/chronic pelvic pain syndrome } \\
\text { Setting: outpatient, Egypt } \\
\text { Intervention: prostatic massage } \\
\text { Comparison: no intervention }\end{array}$} \\
\hline \multirow[t]{2}{*}{ Outcomes } & \multirow{2}{*}{$\begin{array}{l}\text { № of participants } \\
\text { (studies) }\end{array}$} & \multirow{2}{*}{$\begin{array}{l}\text { Quality of the evi- } \\
\text { dence } \\
\text { (GRADE) }\end{array}$} & \multirow{2}{*}{$\begin{array}{l}\text { Relative effect } \\
(95 \% \mathrm{Cl})\end{array}$} & \multicolumn{2}{|c|}{ Anticipated absolute effects* $(95 \% \mathrm{Cl})$} \\
\hline & & & & Risk with control & $\begin{array}{l}\text { Risk difference with } \\
\text { prostatic massage }\end{array}$ \\
\hline $\begin{array}{l}\text { Prostatitis symptoms } \\
\text { assessed with: NIH-CPSI score } \\
\text { Scale from: } 0 \text { to } 43 \\
\text { follow-up: } 4 \text { weeks } \\
\text { Benefit is indicated by lower scores }\end{array}$ & $\begin{array}{l}44 \\
(1 \mathrm{RCT})\end{array}$ & $\begin{array}{l}\oplus \ominus \odot \odot \\
\text { Very low } 1,2\end{array}$ & - & $\begin{array}{l}\text { The mean prostati- } \\
\text { tis symptom score } \\
\text { was } 12.4\end{array}$ & $\begin{array}{l}\text { MD } 1.10 \text { lower } \\
\text { (5.63 lower to } 3.43 \\
\text { higher) }\end{array}$ \\
\hline Adverse events - not reported & - & - & - & - & - \\
\hline Sexual dysfunction - not reported & - & - & - & - & - \\
\hline Quality of life - not reported & - & - & - & - & - \\
\hline Depression and anxiety - not reported & - & - & - & - & - \\
\hline
\end{tabular}




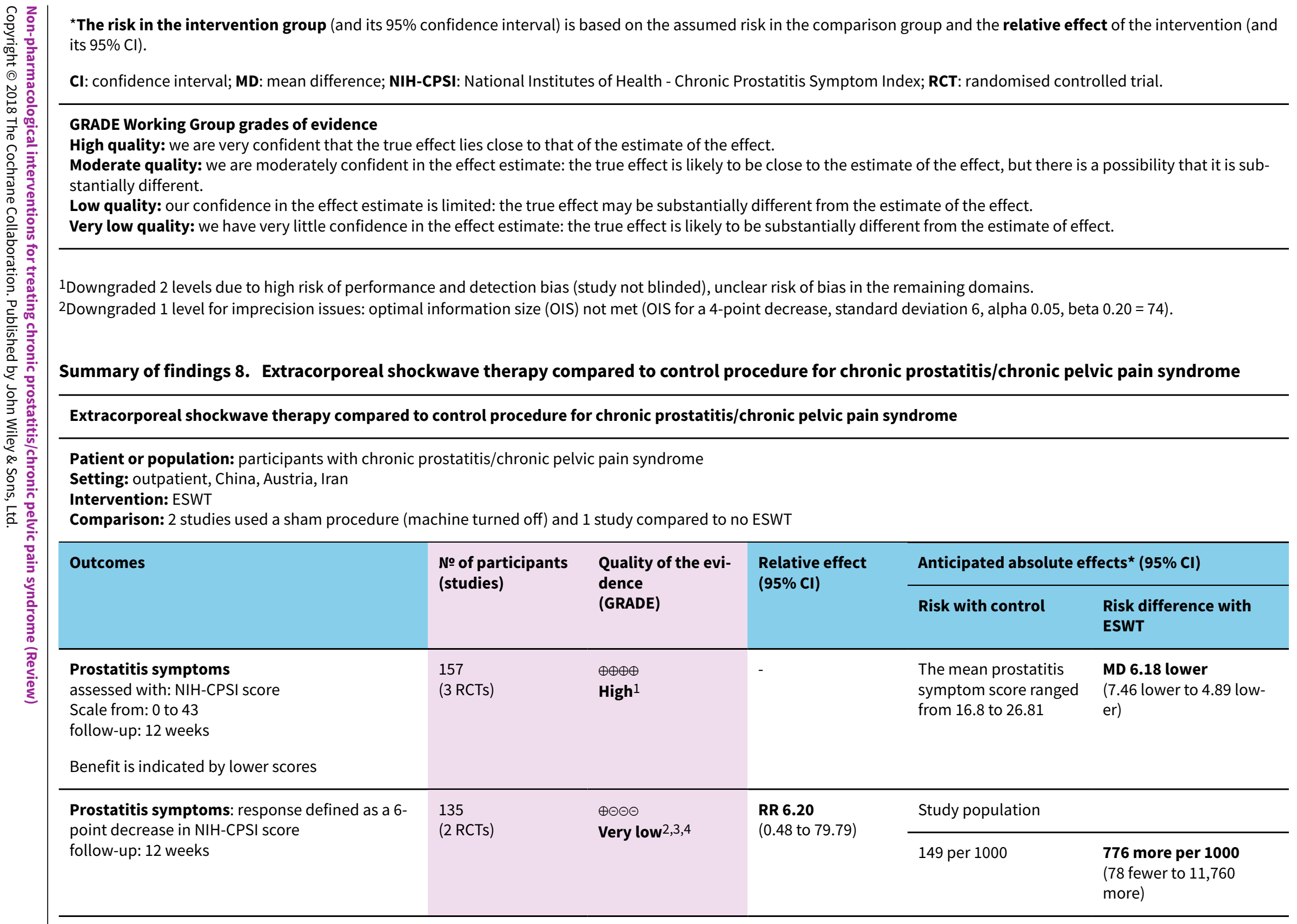




\begin{tabular}{|c|c|c|c|c|c|c|c|}
\hline 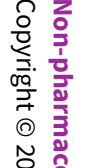 & $\begin{array}{l}\text { Prostatitis symptoms } \\
\text { assessed with: NIH-CPSI score } \\
\text { Scale from: } 0 \text { to } 43 \\
\text { follow-up: } 24 \text { weeks }\end{array}$ & $\begin{array}{l}97 \\
(2 \text { RCTs) }\end{array}$ & $\begin{array}{l}\oplus \oplus \ominus \ominus \\
\text { Low }^{2,5}\end{array}$ & - & $\begin{array}{l}\text { The mean prostatitis } \\
\text { symptom score ranged } \\
\text { from } 16.1 \text { to } 27\end{array}$ & $\begin{array}{l}\text { MD } 2.23 \text { lower } \\
\text { (5.98 lower to } 1.53 \text { high- } \\
\text { er) }\end{array}$ & $\Gamma$ \\
\hline$\frac{20}{2}$ & Adverse events & 195 & $\oplus \oplus \ominus \ominus$ & RR 1.22 & Study population & & \\
\hline 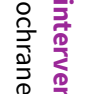 & follow-up: 24 weeks & & & & 93 per 1000 & $\begin{array}{l}\mathbf{2 0} \text { more per } 1000 \\
\text { (38 fewer to } 140 \text { more) }\end{array}$ & 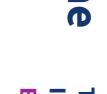 \\
\hline 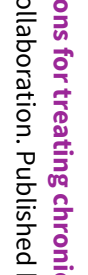 & $\begin{array}{l}\text { Sexual dysfunction } \\
\text { assessed with: International Index of Erectile } \\
\text { Function } \\
\text { Scale from: } 5 \text { to } 25 \\
\text { follow-up: } 12 \text { weeks } \\
\text { Benefit is indicated by higher scores }\end{array}$ & $\begin{array}{l}60 \\
(1 \mathrm{RCT})\end{array}$ & $\begin{array}{l}\oplus \oplus \oplus \ominus \\
\text { Moderate } 6\end{array}$ & - & $\begin{array}{l}\text { The mean sexual dys- } \\
\text { function was } 16.83\end{array}$ & $\begin{array}{l}\text { MD } 3.34 \text { higher } \\
\text { (2.68 higher to } 4 \text { higher) }\end{array}$ & 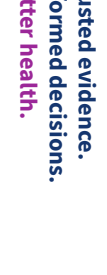 \\
\hline 5 & Quality of life - not reported & - & - & - & - & - & \\
\hline$\frac{1}{n}$ & Depression and anxiety - not reported & - & - & - & - & - & \\
\hline 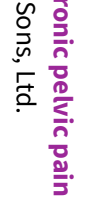 & $\begin{array}{l}{ }^{\star} \text { The risk in the intervention group (and its } 9 \\
\text { its } 95 \% \mathrm{Cl} \text { ). } \\
\text { Cl: confidence interval; ESWT: extracorporeal } \\
\text { randomised controlled trial; RR: risk ratio. }\end{array}$ & snfidence & $\begin{array}{l}\text { lased on the a } \\
\text { ean difference }\end{array}$ & sk in the comp & $\begin{array}{l}\text { roup and the relative ef } \\
\text { f Health - Chronic Prosta }\end{array}$ & $\begin{array}{l}\text { ct of the intervention (and } \\
\text { tis Symptom Index; RCT: }\end{array}$ & \\
\hline 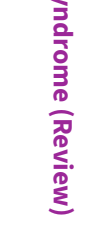 & $\begin{array}{l}\text { GRADE Working Group grades of evidence } \\
\text { High quality: we are very confident that the tr } \\
\text { Moderate quality: we are moderately confide } \\
\text { stantially different. } \\
\text { Low quality: our confidence in the effect estir } \\
\text { Very low quality: we have very little confiden }\end{array}$ & $\begin{array}{l}\text { fect lies cl } \\
\text { the effect } \\
\text { s limited: } \\
\text { the effect }\end{array}$ & $\begin{array}{l}\text { f the estimate } \\
\text { true effect is } \\
\text { ct may be sub } \\
\text { true effect is }\end{array}$ & $\begin{array}{l}\text { ect. } \\
\text { de close to the } \\
\text { different from } \\
\text { De substantially }\end{array}$ & $\begin{array}{l}\text { of the effect, but there } \\
\text { nate of the effect. } \\
\text { t from the estimate of e }\end{array}$ & a possibility that it is sub- & 尺 \\
\hline & $\begin{array}{l}1 \text { Whereas one of the studies was not blinded, wh } \\
\text { with other studies with low risk of bias. } \\
\text { 2Downgraded } 1 \text { level due to risk of bias: one of } \\
\text { 3Downgraded } 1 \text { level due to inconsistency }\left(1^{2}=\right. \\
{ }^{4} \text { Downgraded } 1 \text { level due to imprecision issues: } \\
\text { 5Downgraded } 1 \text { level due to inconsistency }\left(1^{2}=\right. \\
6 \text { Downgraded } 1 \text { level due to imprecision issues: }\end{array}$ & $\begin{array}{l}\text { ould have } \\
\text { dies that } \\
\text { vents and } \\
\text { confidenc }\end{array}$ & $\begin{array}{l}\text { risk of perfor } \\
\text { ents for this ol } \\
\text { ence interval. }\end{array}$ & $\begin{array}{l}\text { d detection bia } \\
\text { as not blinded. }\end{array}$ & not downgrade for ris & bias due to the consistency & 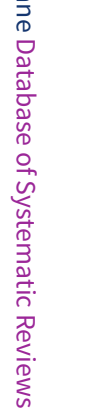 \\
\hline
\end{tabular}




\begin{tabular}{|c|c|c|c|c|c|}
\hline \multicolumn{6}{|c|}{ Transrectal thermotherapy compared to medical treatment for chronic prostatitis/chronic pelvic pain syndrome } \\
\hline \multicolumn{6}{|c|}{$\begin{array}{l}\text { Patient or population: participants with chronic prostatitis/chronic pelvic pain syndrome } \\
\text { Setting: outpatient, China and Korea } \\
\text { Intervention: transrectal thermotherapy } \\
\text { Comparison: medical treatment }\end{array}$} \\
\hline \multirow[t]{2}{*}{ Outcomes } & \multirow{2}{*}{$\begin{array}{l}\text { № of participants } \\
\text { (studies) }\end{array}$} & \multirow{2}{*}{$\begin{array}{l}\text { Quality of the evi- } \\
\text { dence } \\
\text { (GRADE) }\end{array}$} & \multirow{2}{*}{$\begin{array}{l}\text { Relative effect } \\
(95 \% \mathrm{CI})\end{array}$} & \multicolumn{2}{|c|}{ Anticipated absolute effects* $(95 \% \mathrm{Cl})$} \\
\hline & & & & $\begin{array}{l}\text { Risk with medical } \\
\text { treatment }\end{array}$ & $\begin{array}{l}\text { Risk difference with } \\
\text { transrectal ther- } \\
\text { motherapy }\end{array}$ \\
\hline $\begin{array}{l}\text { Prostatitis symptoms } \\
\text { assessed with: NIH-CPSI score } \\
\text { Scale from: } 0 \text { to } 43 \\
\text { follow-up: } 6 \text { to } 12 \text { weeks } \\
\text { Benefit is indicated by lower scores }\end{array}$ & $\begin{array}{l}140 \\
(2 \text { RCTs })\end{array}$ & $\begin{array}{l}\oplus \oplus \Theta \odot \\
\text { Low } 1\end{array}$ & - & $\begin{array}{l}\text { The mean prostatitis } \\
\text { symptom score ranged } \\
\text { from } 14.33 \text { to } 17.19\end{array}$ & $\begin{array}{l}\text { MD } 2.50 \text { lower } \\
\text { (3.82 lower to } 1.18 \\
\text { lower) }\end{array}$ \\
\hline Adverse events - not reported & - & - & - & - & - \\
\hline Sexual dysfunction - not reported & - & - & - & - & - \\
\hline Quality of life - not reported & - & - & - & - & - \\
\hline Depression and anxiety - not reported & - & - & - & - & - \\
\hline \multicolumn{6}{|c|}{$\begin{array}{l}{ }^{*} \text { The risk in the intervention group (and its } 95 \% \text { confidence interval) is based on the assumed risk in the comparison group and the relative effect of the intervention (and } \\
\text { its } 95 \% \mathrm{Cl} \text { ). }\end{array}$} \\
\hline \multicolumn{6}{|c|}{$\begin{array}{l}\text { GRADE Working Group grades of evidence } \\
\text { High quality: we are very confident that the true effect lies close to that of the estimate of the effect. } \\
\text { Moderate quality: we are moderately confident in the effect estimate: the true effect is likely to be close to the estimate of the effect, but there is a possibility that it is sub- } \\
\text { stantially different. } \\
\text { Low quality: our confidence in the effect estimate is limited: the true effect may be substantially different from the estimate of the effect. } \\
\text { Very low quality: we have very little confidence in the effect estimate: the true effect is likely to be substantially different from the estimate of effect. }\end{array}$} \\
\hline
\end{tabular}

${ }^{\star}$ The risk in the intervention group (and its 95\% confidence interval) is based on the assumed risk in the comparison group and the relative effect of the intervention (and its $95 \% \mathrm{Cl}$ ).

\section{GRADE Working Group grades of evidence}

High quality: we are very confident that the true effect lies close to that of the estimate of the effect.

stantially different.

Very low quality: we have very little confidence in the effect estimate: the true effect is likely to be substantially different from the estimate of effect.

${ }^{1}$ Downgraded 2 levels due to high risk of allocation concealment bias, performance and detection bias (study not blinded) and high risk of attrition bias. 


\begin{tabular}{|c|c|c|c|c|c|c|}
\hline \multirow{11}{*}{ 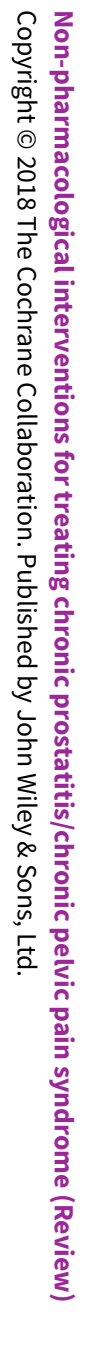 } & \multicolumn{6}{|c|}{$\begin{array}{l}\text { Summary of findings 10. Transrectal thermotherapy (add-on) compared to medical treatment alone for chronic prostatitis/chronic pelvic pain } \\
\text { syndrome }\end{array}$} \\
\hline & \multicolumn{6}{|c|}{ Transrectal thermotherapy (add-on) compared to medical treatment alone for chronic prostatitis/chronic pelvic pain syndrome } \\
\hline & \multicolumn{6}{|c|}{$\begin{array}{l}\text { Patient or population: participants with chronic prostatitis/chronic pelvic pain syndrome } \\
\text { Setting: outpatient, China and Korea } \\
\text { Intervention: transrectal thermotherapy in addition to medical therapy } \\
\text { Comparison: medical treatment alone }\end{array}$} \\
\hline & \multirow[t]{2}{*}{ Outcomes } & \multirow{2}{*}{$\begin{array}{l}\text { № of participants } \\
\text { (studies) } \\
\text { Follow-up }\end{array}$} & \multirow{2}{*}{$\begin{array}{l}\text { Quality of the evi- } \\
\text { dence } \\
\text { (GRADE) }\end{array}$} & \multirow{2}{*}{$\begin{array}{l}\text { Relative effect } \\
(95 \% \mathrm{Cl})\end{array}$} & \multicolumn{2}{|c|}{ Anticipated absolute effects ${ }^{\star}(95 \% \mathrm{Cl})$} \\
\hline & & & & & $\begin{array}{l}\text { Risk with medical } \\
\text { treatment alone }\end{array}$ & $\begin{array}{l}\text { Risk difference with } \\
\text { transrectal ther- } \\
\text { motherapy (add-on) }\end{array}$ \\
\hline & $\begin{array}{l}\text { Prostatitis symptoms } \\
\text { assessed with: NIH-CPSI score } \\
\text { Scale from: } 0 \text { to } 43 \\
\text { follow-up: } 6 \text { to } 12 \text { weeks } \\
\text { Benefit is indicated by lower scores }\end{array}$ & $\begin{array}{l}145 \\
\text { (2 RCTs) }\end{array}$ & $\begin{array}{l}\oplus \oplus \ominus \ominus \\
\text { Low }^{1}\end{array}$ & - & $\begin{array}{l}\text { The mean prostatitis } \\
\text { symptom score ranged } \\
\text { from } 14.33 \text { to } 17.19\end{array}$ & $\begin{array}{l}\text { MD } 4.34 \text { lower } \\
\text { (5.65 lower to } 3.04 \text { low- } \\
\text { er) }\end{array}$ \\
\hline & Adverse events - not reported & - & - & - & - & - \\
\hline & Sexual dysfunction - not reported & - & - & - & - & - \\
\hline & Quality of life - not reported & - & - & - & - & - \\
\hline & Depression and anxiety - not reported & - & - & - & - & - \\
\hline & \multicolumn{6}{|c|}{$\begin{array}{l}{ }^{*} \text { The risk in the intervention group (and its } 95 \% \text { confidence interval) is based on the assumed risk in the comparison group and the relative effect of the intervention (and } \\
\text { its } 95 \% \mathrm{CI} \text { ). }\end{array}$} \\
\hline
\end{tabular}

\section{GRADE Working Group grades of evidence}

High quality: we are very confident that the true effect lies close to that of the estimate of the effect.

Moderate quality: we are moderately confident in the effect estimate: the true effect is likely to be close to the estimate of the effect, but there is a possibility that it is substantially different.

Low quality: our confidence in the effect estimate is limited: the true effect may be substantially different from the estimate of the effect.

Very low quality: we have very little confidence in the effect estimate: the true effect is likely to be substantially different from the estimate of effect. 


\section{B A C K G R O U N D}

\section{Description of the condition}

Prostatitis is a common disorder affecting $10 \%$ to $14 \%$ of men in Europe and the USA (Bajpayee 2012). This health problem motivates $1 \%$ of primary care visits and $8 \%$ of urology consultations in the USA (Collins 1998). Only $5 \%$ to $10 \%$ of prostatitis cases have a bacterial origin (Bartoletti 2007; De La Rosette 1993). This disorder can affect men of all ages and ethnic origins, but it is more common in younger men with a mean age of onset at 42 years old (Schaeffer 2002). The two main clinical features of prostatitis are pelvic pain and lower urinary tract symptoms (LUTS), even though there is a wide range of clinical presentations (Nickel 1999a).

The National Institutes of Health $(\mathrm{NIH})$ classification identifies four types of prostatitis (Nickel 1999a): type I, acute bacterial prostatitis; type II, chronic bacterial prostatitis; type III, chronic prostatitis/chronic pelvic pain syndrome (CP/CPPS) and type IV, asymptomatic prostatitis. It remains unclear whether type III can be linked in all cases to prostatic involvement (True 1999), thus the alternate denomination (CPPS). CP/CPPS is subclassified as type IIIa, inflammatory, and type IIIb, non-inflammatory, depending on the presence of inflammatory cells in prostatic secretions. Before this classification, this entity was denominated chronic abacterial or non-bacterial prostatitis (similar to type IIla CP/CPPS) and prostatodynia (similar to type IIIb CP/CPPS) (Krieger 1996). This change in the classification might have changed the epidemiology of this condition (Krieger 2004).

$\mathrm{CP} / \mathrm{CPPS}$ is defined when pelvic pain is present for at least three of the preceding six months and no other identifiable causes have been detected (Nickel 1999a). Other symptoms include obstructive or irritative voiding difficulties, ejaculatory pain, and haematospermia. Men affected by CP/CPPS have a significantly decreased quality of life ( $Q \circ \mathrm{L})$ and the level of pelvic pain is strongly associated with sexual dysfunction (Trinchieri 2007; Walz 2007). CP/CPPS is associated with other functional somatic syndromes, such as irritable bowel syndrome, interstitial cystitis, chronic fatigue syndrome and fibromyalgia (Rodriguez 2009; Suskind 2013). Diagnosis is usually based on patient history, physical examination, urinalysis and the two- or four-glass test (Nickel 2012). Further investigations are performed when considering differential diagnosis.

There are different theories regarding the aetiology and pathophysiology of CP/CPPS, as follows.

- Infection: bacterial DNA is detected in a significant proportion of men with CP/CPPS (Hou 2012). A previous history of sexually transmitted infection is more frequent in men with CP/CPPS (Pontari 2005). Nevertheless, the isolation of uropathogenic bacteria in prostatic fluids is similar to controls (Nickel 2003a).

- Inflammation/autoimmunity: elevated concentrations of proinflammatory cytokines (interleukin 1, tumour necrosis factor, interferon- $\gamma$ ) and of autoimmunity activity (T-cell proliferation responses to prostate antigens) is found in men with CP/CPPS and in animal models (Pontari 2004).

- Neuropsychological factors: the central nervous system might be involved through several mechanisms of pain sensitisation (Miller 2002; Yang 2003). Increased stress burden, stress response, pain catastrophising cognitions, poor social functioning and psychiatric comorbidity (anxiety and depression) are contributing factors (Riegel 2014).

- Dyssynergic voiding associated with bladder neck hypertrophy is detected in men with refractory CP/CPPS (Dellabella 2006; Hruz 2003). Intraprostatic urinary reflux and increased intraprostatic pressure is associated with inflammation in $\mathrm{CP} /$ CPPS (Kirby 1982; Mehik 2002).

- Other theories described for this condition include: adrenal axis abnormalities (Anderson 2008), pelvic floor muscles dysfunction (Hetrick 2006; Shoskes 2008a), pelvic nerve entrapment (Antolak 2002), genetic predisposition to inflammation (Shoskes 2002), and oxidative stress (Arisan 2006).

\section{Description of the intervention}

There is a wide variety of interventions for treating CP/CPPS, each one addressing a different pathophysiological or symptomatic framework. The diversity of available interventions reflects the complexity of the condition and how little is known about its determinants.

Management of CP/CPPS involves a multimodal and tailored approach (Rees 2015; Shoskes 2008b). Some of the strategies used alone or in combination are the following.

\section{Pharmacological interventions}

- Alpha-blockers.

- 5-alpha reductase inhibitors.

- Antibiotic therapy (quinolones, tetracyclines and other agents).

- Analgesics (non-steroidal anti-inflammatory drugs (NSAIDs), pregabalin).

- Phytotherapy (pollen extract and bioflavonoids).

- Botulinum toxin A.

- Allopurinol.

- Traditional medicine (traditional Chinese medicine, etc.).

- Other pharmacological agents.

\section{Non-pharmacological interventions}

- Acupuncture and electroacupuncture.

- Local thermotherapy.

- Extracorporeal shockwave therapy.

- Electromagnetic chair.

- Myofascial trigger point release.

- Biofeedback.

- Circumcision.

- Lifestyle interventions.

- Physical activity.

- Psychological support.

- Prostatic surgery.

- Other miscellaneous non-pharmacological therapies.

\section{Multimodal approaches}

- Combination therapy: alpha blockers plus antibiotics, antibiotics plus analgesics, etc. 


\section{Adverse events}

Common adverse effects of pharmacological regimens include the following (Brunton 2011).

- Alpha-blockers: hypotension, ejaculatory dysfunction, headache, dizziness and nasal congestion.

- 5-alpha-reductase inhibitors: decreased libido, impotency, and potentiation of hypotension (in combination with alphablockers).

- Quinolones: gastrointestinal discomfort, headache, dizziness, rash and tendinopathy.

- Tetracyclines: gastrointestinal discomfort, rash, teeth discolouration and hepatotoxicity.

- NSAIDs: peripheral oedema, rash, dyspepsia, peptic ulcer and bleeding, renal and hepatic injury, and increased risk of adverse cardiovascular events.

- Phytotherapy: gastrointestinal discomfort and allergic reactions.

The most common adverse effect in physical therapies is pain worsened during or immediately after the procedure (Fitzgerald 2013).

\section{Clinical phenotyping}

Clinical phenotyping is a strategy that was developed to deliver customised treatment in an aetiological framework (Shoskes 2008b). The UPOINT system addresses six domains: Urinary symptoms, Psychosocial dysfunction, Organ-specific findings, Infection, Neurological dysfunction and Tenderness of muscles, and offers an algorithmic approach for the use of the various available interventions. The number of affected domains holds a significant correlation with the prostatitis symptoms score and the addition of a Sexual dysfunction domain (UPOINT(S)) improves accuracy in stratification of symptom severity (Magri 2010). While in itself it is not an intervention, it serves as a screening tool to select the most appropriate intervention for each patient.

\section{How the intervention might work}

\section{Pharmacological interventions}

Alpha-blockers reduce the autonomic sympathetic tone in the bladder neck and prostate, improving urinary flow and LUTS. 5-alpha-reductase inhibitors reduce the production of dihydrotestosterone and, consequently, the size of the prostatic gland dependent on the stimulation of this hormone. This might reduce pain and impaired voiding (Brunton 2011).

NSAIDs are antagonists to the cyclo-oxygenases (COX) enzymes type 1 and 2 and their proinflammatory subproducts (Brunton 2011). Both non-selective and selective (COX-2) inhibitors could therefore decrease inflammatory mediated pain in CP/CPPS.

Phytotherapy includes the use of pollen extract and bioflavonoids that appear to have anti-inflammatory properties, decreasing acinar cell proliferation and the production of interleukin-6, tumour necrosis factor $\alpha$, and other proinflammatory cytokines (Capodice 2005; Kamijo 2001).

Even if CP/CPPS is defined when no bacterial cause can be identified, antibiotics have been used to treat it under the assumption of the existence of an occult or undertreated infection (Hou 2012).

Allopurinol would reduce the prostatic secretions of purine and pyrimidine base-containing metabolites in urine. These metabolites could be responsible for prostatic inflammation through urinary reflux (McNaughton 2002).

Botulinum toxin $\mathrm{A}$ has denervating properties and causes reduction in pain mediators when applied to the prostate in animal models. It also causes apoptosis and involution of the prostate gland (Chuang 2006).

\section{Non-pharmacological interventions}

Acupuncture targets specific cutaneous points representing various internal organs using fine needle insertion and sometimes adding electric current to increase stimulation (electroacupuncture). In animal models, electroacupuncture has anti-inflammatory properties and activates analgesic neurotransmitters (Kim 2006).

Locally induced hyperthermia, using transrectal or transurethral procedures, could decrease oxygen free radicals associated with prostatic inflammation (Gao 2012).

Myofascial trigger point release targets pelvic floor musculature dysfunction as a potential cause or contributor to CP/CPPS (Fitzgerald 2013). Biofeedback also addresses pelvic floor muscle through initial contraction to achieve further relaxation (Capodice 2005).

Extracorporeal shockwave therapy could promote vascularisation of the prostatic tissue and modulate nociceptive nerve impulses and pelvic floor tone (Pajovic 2016).

The length of the foreskin is positively associated with the presence of symptoms of CP/CPPS; therefore, it has been proposed that circumcision could reduce prostatitis symptoms (Zhao 2015).

There are certain risk factors in the lifestyle of men with CP/CPPS, including alcohol consumption and smoking status, among others, that are associated with worse clinical outcomes. Interventions aimed at reducing those risk factors, including those aimed at increasing physical activity, could reduce prostatitis symptoms (Chen 2016).

Prostatic massage has been a classical treatment for CP/CPPS aimed at relieving prostatic congestion, although the mechanisms for its therapeutic effects are controversial (Nickel 1999b).

It has been suggested that psychological treatments could be helpful in all types of chronic pain syndromes and the psychiatric comorbidity associated with the condition (e.g. depression secondary to chronic pain) (Riegel 2014).

\section{Why it is important to do this review}

The Cochrane Urology Group undertook an extensive prioritisation exercise to identify a core portfolio of the most clinically important titles. Consequently, this title was identified as a clinically important priority by the urology expert panel for development, maintenance and investment of resources by the editorial base. 
CP/CPPS is a prevalent condition among men and it causes significant impairment of QoL. There was a previous Cochrane Review on the same subject but with a different methodological approach (McNaughton 2000). Other non-Cochrane systematic reviews were also undertaken in previous years: some of them focused on individual interventions (Qin 2016a; Yang 2006; Zhu 2014), while others had a wider scope of interventions (Anothaisintawee 2011; Cohen 2012; Magistro 2016). We consider that a new and updated Cochrane Review is needed to critically summarise the body of evidence for this complex condition using the GRADE approach, thus providing key information about the best estimate of the magnitude of the effect in relative terms and absolute differences for patient-important outcomes. Previous systematic reviews did not use this approach and had variable adherence to the rigorous methodology recommended by Cochrane.

The protocol for this review was first published in August 2016 with the title 'Interventions for treating chronic prostatitis/chronic pelvic pain syndrome' (Franco 2016). Due to the retrieval of a significant amount of included studies, the review team and the Cochrane Urology Group decided to split the review in two more narrowly defined reviews: 'Non-pharmacological interventions for treating chronic prostatitis/chronic pelvic pain syndrome' and 'Pharmacological interventions for treating chronic prostatitis/ chronic pelvic pain syndrome' (Franco 2017).

\section{O B JECTIVES}

To assess the effects of non-pharmacological therapies for chronic prostatitis/chronic pelvic pain syndrome (CP/CPPS).

\section{METHODS}

\section{Criteria for considering studies for this review}

\section{Types of studies}

We included randomised controlled trials (RCTs) regardless of their publication status or language of publication.

\section{Types of participants}

We included men of all ages, regardless of social condition or ethnic origin, with CP/CPPS according with type III prostatitis of the NIH classification.

If we identified studies in which only a subset of participants was relevant to this review, we included such studies if data were available separately for the relevant subset.

\section{Types of interventions}

We investigated the following comparisons of experimental intervention versus comparator intervention. Concomitant interventions had to be the same in the experimental and comparator groups to establish fair comparisons. We performed a condition-based comprehensive bibliographic search to find all interventions tested so far for CP/CPPS; therefore, some of them might not be listed in this section.

\section{Non-pharmacological interventions}

- Acupuncture and electroacupuncture.

- Circumcision.
- Electromagnetic chair.

- Lifestyle interventions.

- Physical activity.

- Prostatic massage.

- Extracorporeal shockwave therapy.

- Local thermotherapy (transurethral, transrectal thermotherapy and external).

- Biofeedback.

- Myofascial trigger point release.

- Laser therapy.

- Tibial nerve stimulation.

- Myofascial therapy.

- Osteopathy.

- Sono-electromagnetic therapy.

- Transelectrical nerve stimulation

- Transurethral needle ablation.

- Non-intrusive ultrasound.

- Psychological support.

- Prostatic surgery.

- Other miscellaneous non-pharmacological therapies.

\section{Multimodal approaches}

- Combination of pharmacological and non-pharmacological therapy: acupuncture plus antibiotics, local thermotherapy plus alpha-blockers, etc.

- Combination of non-pharmacological therapies.

\section{Comparator interventions}

- Placebo or sham procedure.

- No treatment.

- Other types of interventions: pharmacological and nonpharmacological.

\section{Comparisons}

We performed head-to-head comparisons or intervention versus placebo or sham procedure/no treatment comparisons.

We did not include studies evaluating only pharmacological interventions to avoid overlapping with the review 'Pharmacological interventions for treating chronic prostatitis/ chronic pelvic pain syndrome' (Franco 2017).

\section{Types of outcome measures}

We did not use the measurement of the outcomes assessed in this review as an eligibility criterion.

\section{Primary outcomes}

- Prostatitis symptoms.

- Adverse events.

\section{Secondary outcomes}

- Sexual dysfunction.

- Urinary symptoms.

- Quality of life (QoL)

- Depression and anxiety. 


\section{Method and timing of outcome measurement}

We used clinically important difference for the review outcomes to rate overall quality of the evidence in 'Summary of finding' tables (Johnston 2010). When the mean difference (MD) or risk ratio (RR) was equal to or larger than the minimal clinically important difference (MCID), we assumed that many participants may have gained clinically meaningful improvement from treatment; when the MD was at least half of the MCID but less than the MCID, an appreciable number of participants had likely achieved a clinically meaningful improvement; and when the MD was less than one-half of the MCID, it was unlikely that an appreciable number of participants achieved clinically meaningful improvement (Johnston 2010).

\section{Prostatitis symptoms}

- Measured by the National Institutes of Health - Chronic Prostatitis Symptom Index (NIH-CPSI) as total score and subscore measurements, when possible, and other validated scales.

- We considered an MCID in NIH-CPSI score as a $25 \%$ decrease or a 6-point reduction from baseline (Nickel 2003b). This threshold was used to measure the 'responders rate' (Cates 2015).

\section{Adverse events}

- Defined as treatment intolerance, adverse effects of the interventions at any time after participants were randomised to intervention/comparator groups.

- There was no established threshold for adverse events. We considered the clinically important differences of adverse events above as relative risk reduction of at least 25\% (Guyatt 2011a).

\section{Sexual dysfunction}

- Measured by validated scales (e.g. International Index of Erectile Function, IIEF).

- We considered the MCID in the erectile function domain score of the IIEF of four (Rosen 2011). We planned to use different thresholds of MCID based on the severity of erectile dysfunction, with a threshold of two for men with mild erectile dysfunction, five with moderate erectile dysfunction and seven with severe erectile dysfunction (Rosen 2011). We also considered IIEF-5 of over five points as the MCID (Spaliviero 2010).

\section{Urinary symptoms}

- Measured by IPSS (International Prostate Symptom Score) or AUASS (American Urological Association Symptom Score).

- We considered improvement of the IPSS score of three points as an MCID to assess efficacy and comparative effectiveness (Barry 1995). We planned to use different thresholds of MCID based on the severity of IPSS, with a threshold of three for men with mild LUTS, five for moderate LUTS and eight for severe LUTS (Barry 1995).

\section{Quality of life}

- Assessed by the Medical Outcomes Study Short Form 12 (SF-12) or other validated scales.

- We considered an MCID of SF-12 physical component score to be 8 and SF-12 mental component score to be 4 (Parker 2013).

\section{Depression and anxiety}

- Assessed by Beck Depression Inventory, State Anxiety Inventory$Y$ or other validated scales.

- We considered an MCID of Beck Depression Inventory to be 11 and State Anxiety Inventory-Y to be 10 (Button 2015; Corsaletti 2014).

We considered outcomes measured up to and including 12 months after randomisation as short-term, and later than 12 months as long-term.

\section{Main outcomes for 'Summary of findings' tables}

We presented 'Summary of findings' tables reporting the following outcomes listed according to priority.

- Prostatitis symptoms.

- Adverse events.

- Sexual dysfunction.

- QoL.

- Depression and anxiety.

\section{Search methods for identification of studies}

We searched for all published and unpublished RCTs meeting our stated inclusion/exclusion criteria, without restrictions on language, publication date or publication status, and in consultation with the Cochrane Urology Information Specialist.

\section{Electronic searches}

We identified published, unpublished and ongoing studies by searching the following databases from their inception.

- Cochrane Central Register of Controlled Trials (CENTRAL; 2017, Issue 7) in the Cochrane Library.

- PubMed (1946 to 11 August 2017).

- Embase Elsevier (1947 to 11 August 2017).

- PsycINFO Ovid (1887 to 11 August 2017).

- CINAHL EBSCO (1937 to 11 August 2017).

- ClinicalTrials.gov (www.clinicaltrials.gov, 14 August 2017)

- ISRCTN Registry (BioMed Central; www.isrctn.com/, 14 August 2017).

- World Health Organization International Clinical Trials Registry Platform (www.who.int/trialsearch, 14 August 2017).

The search strategies for databases were modelled on the search strategy designed for PubMed (Appendix 1; Appendix 2; Appendix 3; Appendix 4; Appendix 5; Appendix 6). The PubMed search utilised the Cochrane Highly Sensitive Search Strategy for identifying RCTs in MEDLINE: sensitivity maximising version (2008 revision; Lefebvre 2011). The Embase search utilised the trial filter for therapy, maximising sensitivity developed by the Health Information Research Unit (HIRU) at McMaster University, adapted from Ovid to the Elsevier interface (HIRU 2015). For CENTRAL and clinical trials registries, filters were not applicable. We did not use filters for PsycINFO and CINAHL because the results likely to be obtained were very few.

\section{Searching other resources}

We tried to identify other potentially eligible trials or ancillary publications by searching the reference lists of retrieved included 
trials and relevant reviews, meta-analyses and health technology assessment reports. We contacted authors of included studies to identify any further studies that we may have missed. We contacted drug and device manufacturers for ongoing or unpublished trials. We searched abstract proceedings of the American Urological Association, European Association of Urology and Society of Sexual Medicine from 2015 to 2017 for unpublished studies (Appendix 7).

We searched other grey literature sources such as:

- Open Grey (www.opengrey.eu/);

- New York Academy of Medicine Grey Literature Report (www.greylit.org/);

- Google Scholar.

\section{Data collection and analysis}

\section{Selection of studies}

We used reference management software (EndNote) and Covidence to identify and remove duplicate records. Three review authors (JVAF, TT, VV) independently scanned in pairs the abstract, title, or both, of remaining records retrieved, to determine which studies should be assessed further. Five review authors (JVAF, TT, SI, YX, VV) investigated all potentially relevant records as full text, mapped records to studies, and classified studies as included studies, excluded studies, studies awaiting classification or ongoing studies in accordance with the criteria for each provided in the Cochrane Handbook for Systematic Reviews of Interventions (Higgins 2011a). We used Covidence for title/abstract, and full-text screening. We resolved any discrepancies through consensus or recourse to a third review author $(\mathrm{JHJ})$. If resolution of a disagreement was not possible, we designated the study as 'awaiting classification' (Characteristics of studies awaiting classification) and we contacted study authors for clarification. We documented reasons for exclusion of studies that may have reasonably been expected to be included in the review in a Characteristics of excluded studies table. We presented an adapted PRISMA flow diagram showing the process of study selection (Liberati 2009).

\section{Data extraction and management}

We developed a dedicated data abstraction form that we pilot tested ahead of time.

For studies that fulfilled inclusion criteria, six review authors (JVAF, $\mathrm{VV}, \mathrm{TT}, \mathrm{SI}, \mathrm{YX}, \mathrm{JHJ}$ ) independently abstracted in pairs the following information, which is provided in the Characteristics of included studies table.

- Study design.

- Study dates (if dates were not available then this was reported as such).

- Study settings and country.

- Participant inclusion and exclusion criteria.

- Participant details, baseline demographics.

- Number of participants by study and by study arm.

- Details of relevant experimental and comparator interventions such as dose, route, frequency and duration.

- Definitions of relevant outcomes, and method and timing of outcome measurement as well as any relevant subgroups.
- Study funding sources.

- Declarations of interest by primary investigators.

We further summarised some of the characteristics of the studies, participants and interventions in additional tables (Table 1; Table 2).

We extracted outcomes data relevant to this Cochrane Review as needed for calculation of summary statistics and measures of variance. For dichotomous outcomes, we attempted to obtain numbers of events and totals of population for a $2 \times 2$ table, as well as summary statistics with corresponding measures of variance. For continuous outcomes, we attempted to obtain means and standard deviations or data necessary to calculate this information. We resolved any disagreements by discussion, or, if required, by consultation with a third review author (SI or JHJ).

We provided information, including trial identifier, about potentially relevant ongoing studies in a Characteristics of ongoing studies table. We attempted to contact authors of included studies to obtain key missing data as needed.

\section{Dealing with duplicate and companion publications}

In the event of duplicate publications, companion documents or multiple reports of a primary study, we maximised yield of information by mapping all publications to unique studies and collating all available data. We used the most complete data set aggregated across all known publications. In case of doubt, we gave priority to the publication reporting the longest follow-up associated with our primary or secondary outcomes.

\section{Assessment of risk of bias in included studies}

Six review authors (JVAF, VV, TT, SI, YX, JHJ) assessed the risk of bias of each included study independently in pairs. We resolved disagreements by consensus, or by consultation with a third review author (JVAF or VV).

We assessed risk of bias using the Cochrane 'Risk of bias' assessment tool (Higgins 2011b). We assessed the following domains.

- Random sequence generation (selection bias).

- Allocation concealment (selection bias).

- Blinding of participants and personnel (performance bias).

- Blinding of outcome assessment (detection bias).

- Incomplete outcome data (attrition bias).

- Selective reporting (reporting bias).

- Other sources of bias.

We judged the risk of bias domains as 'low risk,' 'high risk' or 'unclear risk' and evaluated individual bias items as described in the Cochrane Handbook for Systematic Reviews of Interventions (Higgins 2011b). We presented a 'Risk of bias' summary figure to illustrate these findings.

For performance bias (blinding of participants and personnel) and detection bias (blinding of outcome assessment), we evaluated the risk of bias separately for each outcome, and we grouped outcomes according to whether they were measured subjectively or objectively when reporting our findings in the 'Risk of bias' table. However, all end points were subjective outcomes. 
We assessed attrition bias (incomplete outcome data) on an outcome-specific basis, and grouped outcomes with like judgements when reporting our findings in the 'Risk of bias' table.

We further summarised the risk of bias across domains for each outcome in each included study, as well as across studies and domains for each outcome.

\section{Measures of treatment effect}

We expressed dichotomous data as risk ratios (RR) with 95\% confidence intervals $(\mathrm{Cls})$. We expressed continuous data as mean differences (MD) with 95\% Cls unless different studies used different measures to assess the same outcome, in which case we expressed data as standardised mean differences (SMD) with $95 \% \mathrm{Cls}$.

\section{Unit of analysis issues}

The unit of analysis was the individual participant. If we identified cross-over trials, cluster-randomised trials or trials with more than two intervention groups for inclusion in the review, we handled these in accordance with guidance provided in the Cochrane Handbook for Systematic Reviews of Interventions (Higgins 2011c).

\section{Dealing with missing data}

We obtained missing data from study authors, if feasible, and performed intention-to-treat analyses if data were available; otherwise, we performed available-case analyses. We investigated attrition rates, such as dropouts, losses to follow-up and withdrawals, and we critically appraised issues of missing data. We did not impute missing data.

\section{Assessment of heterogeneity}

In the event of excessive heterogeneity unexplained by subgroup analyses, we did not report outcome results as the pooled effect estimate in a meta-analysis, but we provided a narrative description of the results of each study.

We identified heterogeneity (inconsistency) through visual inspection of the forest plots to assess the amount of overlap of Cls, and the $\mathrm{I}^{2}$ statistic, which quantifies inconsistency across studies to assess the impact of heterogeneity on the meta-analysis (Higgins 2002; Higgins 2003). We interpreted the $\mathrm{I}^{2}$ statistic as follows.

- $0 \%$ to $40 \%$ : may not be important.

- $30 \%$ to $60 \%$ : may indicate moderate heterogeneity.

- $50 \%$ to $90 \%$ : may indicate substantial heterogeneity.

- $75 \%$ to $100 \%$ : considerable heterogeneity.

When we found heterogeneity, we attempted to determine possible reasons for it by examining individual study and subgroup characteristics.

\section{Assessment of reporting biases}

We attempted to obtain study protocols to assess for selective outcome reporting.

If we had included 10 studies or more investigating a particular outcome, we would have used funnel plots to assess small-study effects. Several explanations can be offered for the asymmetry of a funnel plot, including true heterogeneity of effect with respect to trial size, poor methodological design (and hence bias of small trials) and publication bias. Therefore, we would have interpreted the results carefully.

\section{Data synthesis}

Unless there was good evidence for homogeneous effects across studies, we summarised data using a random-effects model. We interpreted random-effects meta-analyses with due consideration of the whole distribution of effects. In addition, we performed statistical analyses according to the statistical guidelines contained in the Cochrane Handbook for Systematic Reviews of Interventions (Higgins 2011a). For dichotomous outcomes, we used the MantelHaenszel method; for continuous outcomes, we used the inverse variance method. We used Review Manager 5 (RevMan 2014) software to perform analyses.

\section{Subgroup analysis and investigation of heterogeneity}

We expected the following characteristics to introduce clinical heterogeneity, and planned to carry out subgroup analyses with investigation of interactions.

- Participants' characteristics: symptom severity at recruitment, age, presence of clinical comorbidities (irritable bowel syndrome, fibromyalgia, interstitial cystitis).

- Duration of the intervention: depending on intervention type, measured in sessions (e.g. one session or repeated sessions) or weeks (e.g. less than 12 weeks or more than 12 weeks).

We planned to use the test for subgroup differences in Review Manager 5 to compare subgroup analyses if there had been sufficient studies (RevMan 2014).

\section{Sensitivity analysis}

We planned to perform sensitivity analyses to explore the influence of the following factors (when applicable) on effect sizes.

- Restricting the analysis by taking into account risk of bias, by excluding studies at 'high risk' or 'unclear risk.'

- Explore the impact of re-expressing symptom severity as a dichotomous outcome.

- Excluding studies that included participants with a diagnosis of chronic non-bacterial prostatitis or prostatodynia, not filling the criteria of the 1999 Research Consensus (Nickel 1999a).

\section{'Summary of findings' tables}

We presented the overall quality of the evidence for each outcome according to the GRADE approach, which takes into account five criteria related to internal validity (risk of bias, inconsistency, imprecision, publication bias), and external validity, such as directness of results (Guyatt 2008). For each comparison, two review authors (JVAF, JHJ) independently rated the quality of evidence for each outcome as 'high,' 'moderate,' 'low' or 'very low' using GRADEpro GDT. We resolved any discrepancies by consensus, or, if needed, by arbitration by a third review author (VV). We presented a summary of the evidence for the main outcomes in the 'Summary of findings' tables, which provide key information about: the best estimate of the magnitude of the effect in relative terms and absolute differences for each relevant comparison of alternative management strategies; numbers of participants and studies addressing each important outcome and the rating of the overall confidence in effect estimates for each outcome (Guyatt 2011b; Schünemann 2011). If meta-analysis was not possible, we 
presented results in a narrative 'Summary of findings' table. We initially planned to present 'Summary of findings' tables for all comparisons, however, given their multiplicity, we have presented only those most related to clinical practice or containing at least two clinical trials in order to highlight the evidence most relevant to clinicians, patients and other stakeholders (see Differences between protocol and review). Nevertheless, all comparisons were rated using the GRADE approach and are available under the section Effects of interventions.

We used the controlled vocabulary suggested by Glenton 2010 to summarise the findings of the 'Summary of findings' tables in the 'Plain language summary.'

\section{RES U L T S}

\section{Description of studies}

\section{Results of the search}

For detailed information of the results of the search see Figure 1. This review shares the search strategy for the protocol
'Pharmacological Interventions for treating chronic prostatitis/ chronic pelvic pain syndrome' (Franco 2017). In this section, we described the study flow for the studies relevant to the review question. For this review, we screened 1500 records after removing duplicates. We included two studies from other systematic reviews on this topic (McNaughton 2000). We excluded 1386 records and screened 130 records in the full-text assessment. We excluded 73 studies (76 records) after full-text assessment (see Characteristics of excluded studies table). The search identified one protocol of a completed study (Rochester 2011), but we could not retrieve study results (see Characteristics of studies awaiting classification table). We identified four ongoing studies (see Characteristics of ongoing studies table). We included 38 studies (49 records) in this review. 
Figure 1. Study flow diagram.

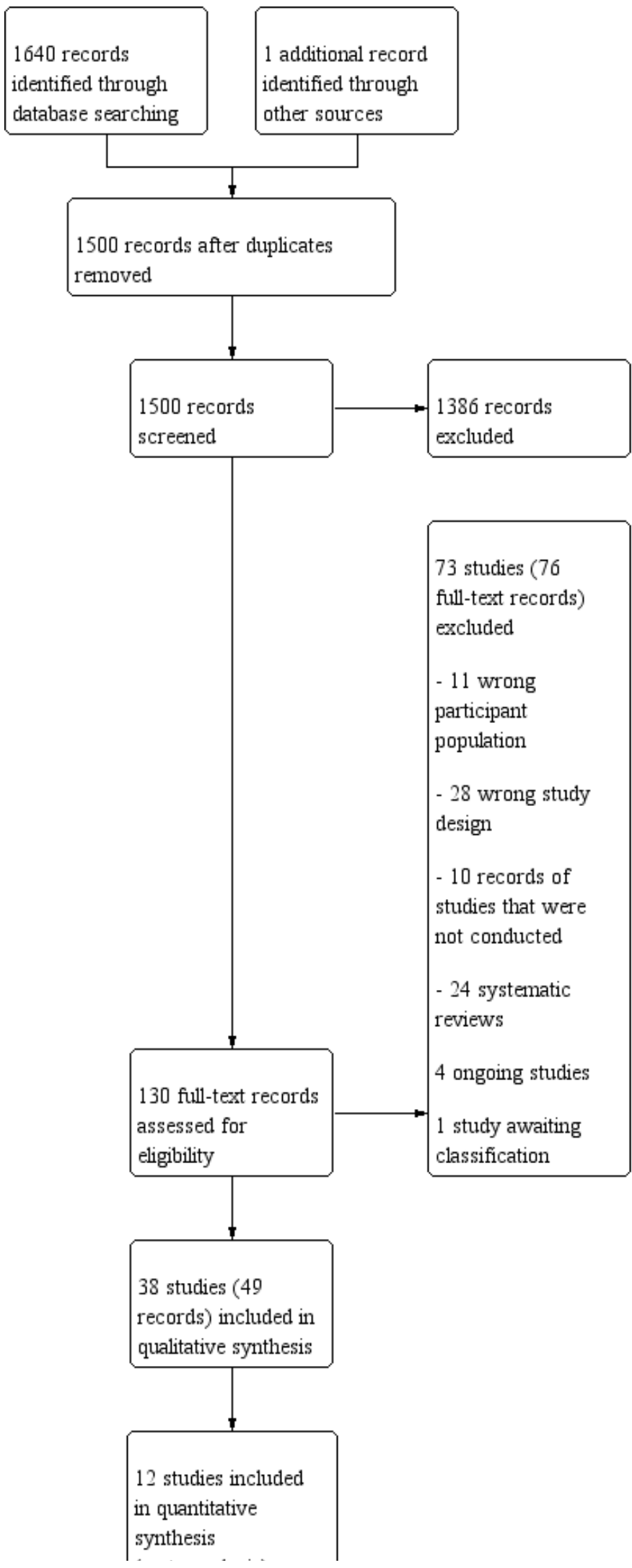


Figure 1. (Continued)

st quasumuv
synthesis
(meta-analysis)

\section{Included studies}

We included 38 studies (see Characteristics of included studies table).

\section{Design}

All the included studies were RCTs.

\section{Sample sizes}

Median sample size was 60 (interquartile range 35 to 100). The smallest sample size was 20 and the largest sample size was 774 .

\section{Setting}

Since CP/CPPS is usually treated in an outpatient setting, most studies offered ambulatory care. Some studies, depending on the type of intervention (see below), required a temporary stay in hospital, particularly those studies that used therapeutic devices. The studies were conducted in Egypt (Ateya 2006; Samhan 2011), China (Chen 2009; Fang 2005; Gao 2012; Kaikai 2014; Shen 2006; Wang 2002; Yang 2011; Zeng 2012; Zhang 2011a; Zhao 2015), US (Fitzgerald 2013), Italy (Gallo 2014; Giubilei 2007; Montorsi 1993; Muraro 1995), Switzerland (Kessler 2014), Turkey (Kabay 2009; Kucuk 2015; Sahin 2015), Korea (Lee 2009; Oh 2009; Paick 2006; Yoo 2009), Finland (Leskinen 2002), Germany (Marx 2009), Canada (Nickel 1996), Montenegro (Pajovic 2016), UK (Rowe 2005; Shah 1993), Nigeria (Sikiru 2008), Iran (Vahdatpour 2013), Russia (Neimark 2016; Vassily 1999), and Austria (Zimmermann 2009). Two studies were conducted in more than one country (Kastner 2004; Lee 2008).

\section{Participants}

The median age of participants was 37 years. Three studies did not provide information regarding age (Oh 2009; Shah 1993; Vassily 1999). These studies were reported in abstract form with few data available. The included studies (except Kastner 2004) did not include participants over 50 years old to avoid symptom overlap with benign prostate hyperplasia. Kastner 2004 included participants with a mean age of 60 years.

All studies referred to diagnostic criteria aimed at the differentiation of CP/CPPS from other forms of prostatitis and other urological diseases. Participants underwent digital rectal examination, urine cultures and 2 or 4 glass Meares-Stamey test. They excluded participants who had recently undergone prostatic biopsy or surgery, participants with prostate cancer, participants with a recent history of sexually transmitted diseases and participants with concomitant neurological disorders or severe systemic disorders.

Only one study included participants who had not received other previous treatment (Kucuk 2015). Eleven studies specified that participants had previously received medical treatment with antibiotics or alpha blockers (or both) and had not had a positive response (Fitzgerald 2013; Giubilei 2007; Kaikai 2014; Kastner 2004; Kessler 2014; Lee 2008; Montorsi 1993; Nickel 1996; Rowe
2005; Sahin 2015; Yang 2011). The other studies did not specify whether the participants had received previous treatments for this condition. Nevertheless, a common inclusion criterion included a washout period, as stated in a protocol for medical therapy often cited as a consensus for inclusion/exclusion criteria (Propert 2002).

\section{Interventions}

We included studies assessing a wide variety of nonpharmacological interventions.

- Acupuncture and electroacupuncture (Chen 2009; Kucuk 2015; Lee 2008; Lee 2009; Sahin 2015).

- Local thermotherapy (Gao 2012; Kastner 2004; Leskinen 2002; Montorsi 1993; Muraro 1995; Nickel 1996; Oh 2009; Shah 1993; Vassily 1999; Wang 2002; Yoo 2009).

- Extracorporeal shockwave therapy (Pajovic 2016; Vahdatpour 2013; Zeng 2012; Zimmermann 2009).

- Myofascial trigger point release compared to control intervention (Fitzgerald 2013).

- Biofeedback with or without electrical stimulation compared to control (Yang 2011).

- Psychological support: we found no studies for this intervention.

- Prostatic surgery: we found no studies for this intervention.

- Other miscellaneous non-pharmacological therapies:

* circumcision compared to waiting list (Zhao 2015);

* electromagnetic chair compared to sham procedure (Paick 2006; Rowe 2005);

* laser therapy compared to medical treatment (Fang 2005);

* lifestyle modifications (Gallo 2014);

* osteopathy (Marx 2009);

* physical activity (Giubilei 2007);

* prostatic massage (Ateya 2006; Shen 2006);

* sono-electromagnetic therapy (Kessler 2014);

* TaiJiQuan (Zhang 2011a);

* transelectrical nerve stimulation (TENS) (Samhan 2011; Sikiru 2008);

* tibial nerve stimulation (Kabay 2009);

* ultrasound (Kaikai 2014);

* hypercapnic hypoxia (Neimark 2016).

\section{Outcomes}

Almost all studies reported the effects of the interventions on prostatitis symptoms. All but four studies used the NIH-CPSI score. Leskinen 2002; Nickel 1996; and Rowe 2005 used a 100-point validated scale (Prostatitis Symptom Severity Index) and Wang 2002 used another validated scale (0 to 12, from Neal 1994).

Other secondary outcomes relevant to this review were reported inconsistently. Five studies did not report any of the prespecified outcomes for this review: three studies were only available as abstracts (Oh 2009; Shah 1993; Vassily 1999), one study reported the evolution of prostatitis symptoms with categorical variables 
(Muraro 1995), and one study reported global improvement as a composite outcome of symptoms and laboratory findings (Zhang 2011a). One study described the evolution of prostatitis symptoms with categorical variables; however, it reported the incidence of adverse events (Montorsi 1993).

We found only short-term outcomes for all comparisons.

\section{Funding sources}

Most studies (28 studies, $76 \%$ ) did not specify their funding sources. Three studies received funding from the companies that manufactured the device under evaluation (Kessler 2014; Neimark 2016; Rowe 2005). Three studies stated that they received no funding (Leskinen 2002; Montorsi 1993; Pajovic 2016). Four studies received funding from public institutions (Fitzgerald 2013; Lee 2008; Lee 2009; Zhao 2015).

\section{Excluded studies}

We excluded 73 studies for the following reasons (see Characteristics of excluded studies).

Eleven studies evaluated a wrong participant population: eight studies included participants with bacterial prostatitis, with no disaggregated data for CP/CPPS (Barbalias 1998; Feng 2011; Galeone 2012; Glybochko 2014; Golubchikov 2005; Lokshin 2010; Pushkar' 2006; Simmons 1985), and three studies did not use the NIH criteria for CP/CPPS (Nickel 2011; Zhang 2011b; Zhou 2017).

We found 28 studies to have a wrong study design: 25 studies specified that they did not use randomisation or used a nonrandom sequence for the allocation of participants (Aliaev 2006; Allen 2017; Colleen 1975; DRKS00009352; Evliyaoglu 2002; Hong 2008; Ikeuchi 1990; ISRCTN43221600; Kalinina 2015; Kamalov 2006;
Kogan 2010; Lee 2006; Leng 2007; Lopatkin 2009; Loran 2003; Ma 2015; Osborn 1981; Pavone 2010; Razumov 2005; Stamatiou 2014; Takahashi 2005; Thin 1983; Tkachuk 2006; Tkachuk 2011; Xu 2004); two studies reported the follow-up of a single arm of RCTs (Kotarinos 2009; Marx 2013); one study was a phase II dose-finding study with an adaptive design (Wagenlehner 2017).

Additionally, 10 studies were terminated and there were no outcome data available, due to problems in their conduct (Bschleipfer 2007; NCT00194597; NCT00194623; NCT00194636; NCT00301405; NCT00464373; NCT00529386; NCT01678911; NCT01830829; NCT02042651).

Our search strategy identified 24 systematic reviews that we searched for additional studies and some of them were used in the discussion (Aboumarzouk 2012; Anothaisintawee 2011; Capodice 2005; Chambo 2009; Chang 2016; Chen 2006; Chuang 2006; Cohen 2012; Erickson 2008; Jimenez-Pacheco 2014; Le 2011; Lee 2007; Liu 2016; Magistro 2016; McNaughton 2000; McNaughton 2001; McNaughton 2002; Mishra 2008; Posadzki 2012; Qin 2016a; Qin 2016b; Thakkinstian 2012; Yang 2006; Yang 2008).

\section{Risk of bias in included studies}

See Figure 2 for a summary of risk of bias assessments. See Figure 3 for the individual assessments of the included studies. Detailed description of the supporting judgements can be found in the Characteristics of included studies table. Considering a global assessment of risk of bias for the main outcomes of this review, only one study had low risk of bias (Kessler 2014), 10 studies had unclear risk of bias (Lee 2008; Lee 2009; Leskinen 2002; Nickel 1996; Oh 2009; Sahin 2015; Samhan 2011; Vahdatpour 2013; Vassily 1999; Zimmermann 2009), and the remaining 26 studies had at least one domain with high risk of bias.

\section{Figure 2. Risk of bias graph: review authors' judgements about each risk of bias item presented as percentages} across all included studies.

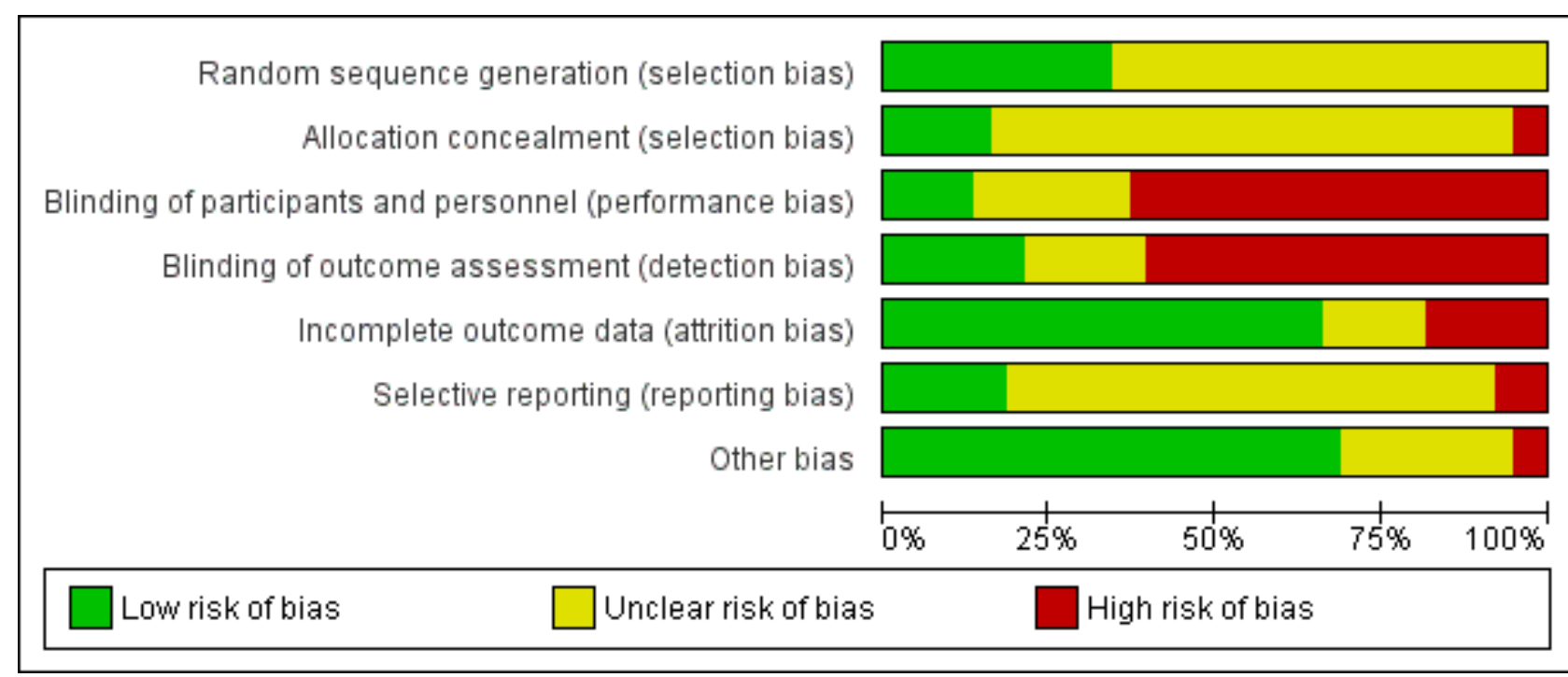


Figure 3. Risk of bias summary: review authors' judgements about each risk of bias item for each study.

\begin{tabular}{|c|c|c|c|c|c|c|c|}
\hline & 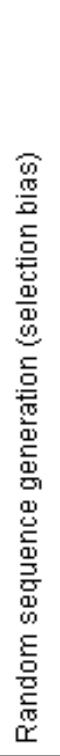 & 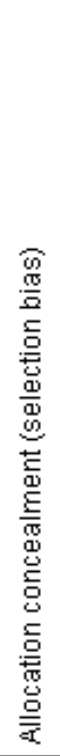 & 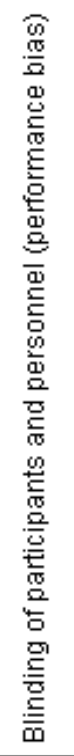 & 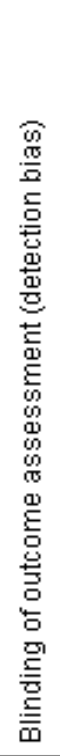 & 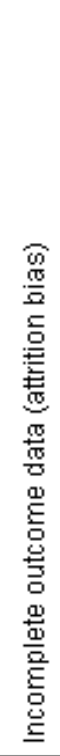 & 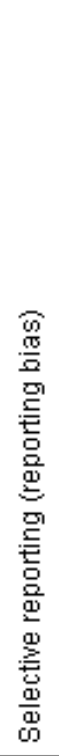 & 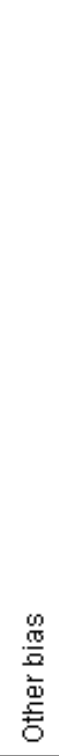 \\
\hline Ateya 2006 & $?$ & $?$ & 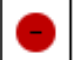 & - & $?$ & $?$ & $?$ \\
\hline Chen 2009 & $\odot$ & $?$ & $\theta$ & $\theta$ & + & $?$ & + \\
\hline Fang 2005 & $?$ & $?$ & $\odot$ & $\odot$ & + & $?$ & + \\
\hline Fitzgerald 2013 & $\odot$ & $\odot$ & $\odot$ & $\odot$ & $\odot$ & $\odot$ & 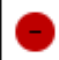 \\
\hline Gallo 2014 & $?$ & $\Theta$ & $\theta$ & - & $\theta$ & $\odot$ & $?$ \\
\hline Gao 2012 & $?$ & $?$ & $\odot$ & $\odot$ & $\odot$ & $?$ & + \\
\hline Giubilei 2007 & $?$ & $?$ & 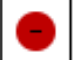 & $\odot$ & 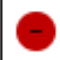 & $?$ & + \\
\hline Kabay 2009 & $?$ & $?$ & $\odot$ & $\odot$ & + & $?$ & + \\
\hline Kaikai 2014 & $?$ & $?$ & $\odot$ & $\odot$ & + & $?$ & + \\
\hline Kastner 2004 & $\oplus$ & $\oplus$ & $?$ & + & 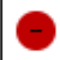 & $?$ & + \\
\hline Kessler 2014 & $\odot$ & $\odot$ & + & $\odot$ & + & $\odot$ & + \\
\hline Kucuk 2015 & $?$ & $?$ & 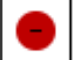 & $\odot$ & + & $?$ & + \\
\hline Lee 2008 & $\odot$ & $?$ & + & + & + & + & + \\
\hline Lee 2009 & $\oplus$ & $?$ & + & $\oplus$ & + & $?$ & + \\
\hline Leskinen 2002 & $?$ & $?$ & $?$ & $?$ & + & $?$ & $?$ \\
\hline Marx 2009 & $\oplus$ & $\oplus$ & $\odot$ & $\odot$ & 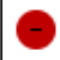 & $?$ & + \\
\hline Montorsi 1993 & $?$ & $?$ & $\odot$ & $\odot$ & + & $?$ & $?$ \\
\hline Muraro 1995 & + & $?$ & $\theta$ & $\Theta$ & $?$ & $?$ & + \\
\hline Neimark 2016 & $?$ & $?$ & $\odot$ & $\odot$ & + & $?$ & $?$ \\
\hline Nickel 1996 & $?$ & $?$ & $?$ & + & + & $?$ & $?$ \\
\hline
\end{tabular}


Figure 3. (Continued)

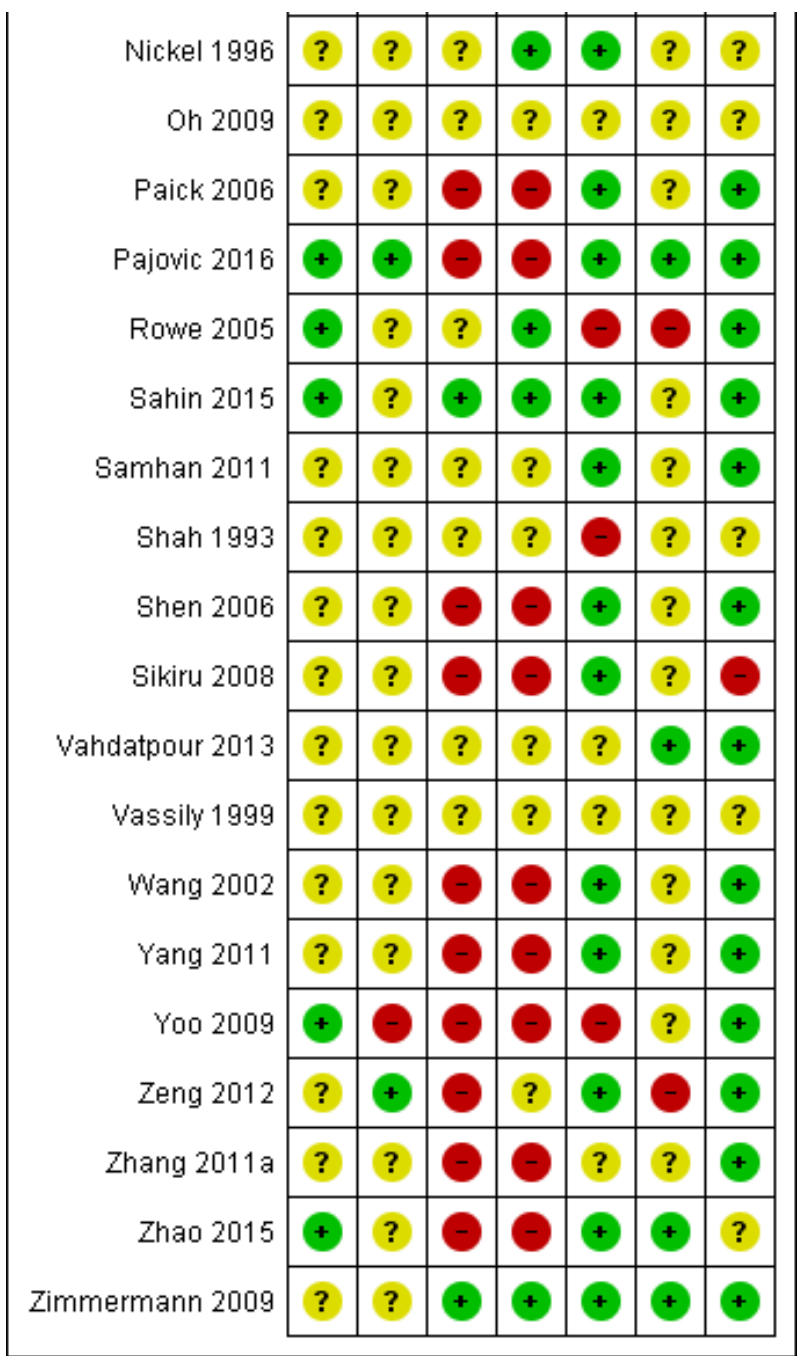

\section{Allocation}

\section{Random sequence generation}

Thirteen studies specified an adequate method of random sequence generation (Chen 2009; Fitzgerald 2013; Kastner 2004; Kessler 2014; Lee 2008; Lee 2009; Marx 2009; Muraro 1995; Pajovic 2016; Rowe 2005; Sahin 2015; Yoo 2009; Zhao 2015). The remaining studies were at unclear risk of bias of random sequence generation.

\section{Allocation concealment}

Six studies specified an adequate method of allocation concealment (Fitzgerald 2013; Kastner 2004; Kessler 2014; Marx 2009; Pajovic 2016; Zeng 2012). Two studies specified that they did not conceal the allocation of participants and were deemed at high risk of bias in this domain (Gallo 2014; Yoo 2009). The remaining studies were at unclear risk of bias in allocation concealment.

\section{Blinding}

\section{Blinding of participants and personnel}

Five studies specified an adequate method for the blinding of participants and personnel (Kessler 2014; Lee 2008; Lee 2009; Sahin 2015; Zimmermann 2009). Twenty-four studies did not adequately blind participants or personnel and were deemed at high risk of bias, considering that all outcomes were subjective (Ateya 2006; Chen 2009; Fang 2005; Fitzgerald 2013; Gallo 2014; Gao 2012; Giubilei 2007; Kabay 2009; Kaikai 2014; Kucuk 2015; Marx 2009; Montorsi 1993; Muraro 1995; Neimark 2016; Paick 2006; Pajovic 2016; Shen 2006; Sikiru 2008; Wang 2002; Yang 2011; Yoo 2009; Zeng 2012; Zhang 2011a; Zhao 2015). The remaining studies were at unclear risk of bias of blinding of participants and personnel.

\section{Blinding of outcome assessment}

All the outcomes of this review were participant-reported outcomes. Eight studies reported blinding of participants (outcome-assessors) (Kastner 2004; Kessler 2014; Lee 2008; Lee 2009; Nickel 1996; Rowe 2005; Sahin 2015; Zimmermann 2009). Twenty-three studies did not adequately blind participants (Ateya 2006; Chen 2009; Fang 2005; Fitzgerald 2013; Gallo 2014; Gao 2012; Giubilei 2007; Kabay 2009; Kaikai 2014; Kucuk 2015; Marx 2009; Montorsi 1993; Muraro 1995; Neimark 2016; Paick 2006; Pajovic 2016; Shen 2006; Sikiru 2008; Wang 2002; Yang 2011; Yoo 2009; Zhang 2011a; Zhao 2015). The remaining studies were at unclear risk of bias of outcome assessment. 


\section{Incomplete outcome data}

Twenty-five studies specified that outcome data for all outcomes were available in all or nearly all participants (Chen 2009; Fang 2005; Fitzgerald 2013; Gao 2012; Kabay 2009; Kaikai 2014; Kessler 2014; Kucuk 2015; Lee 2008; Lee 2009; Leskinen 2002; Montorsi 1993; Neimark 2016; Nickel 1996; Paick 2006; Pajovic 2016; Sahin 2015; Samhan 2011; Shen 2006; Sikiru 2008; Wang 2002; Yang 2011; Zeng 2012; Zhao 2015; Zimmermann 2009). Seven studies had unbalanced or high attrition (or both) of outcome data at followup and were deemed at high risk of bias (Gallo 2014; Giubilei 2007; Kastner 2004; Marx 2009; Rowe 2005; Shah 1993; Yoo 2009). The remaining studies were at unclear risk of bias in this domain.

\section{Selective reporting}

Seven studies had low risk of reporting bias when comparing their outcomes to their protocols or trial registries (Fitzgerald 2013; Kessler 2014; Lee 2008; Pajovic 2016; Vahdatpour 2013; Zhao 2015; Zimmermann 2009). Three studies reported some of their outcomes graphically or with missing data and were deemed at high risk of bias (Gallo 2014; Rowe 2005; Zeng 2012). The remaining studies were at unclear risk of reporting bias.

\section{Other potential sources of bias}

Two studies were at high risk of bias due to large baseline difference in mean symptom scores between groups (Fitzgerald 2013; Sikiru 2008; see Table 2). Seven studies lacked baseline characteristics of participants (Ateya 2006; Montorsi 1993; Neimark 2016; Nickel 1996; Oh 2009; Shah 1993; Vassily 1999), one study had some baseline differences in symptom scores (Leskinen 2002), and two studies did not specify if participants received some additional interventions planned in the protocol (Gallo 2014) or how many participants received the planned intervention (Zhao 2015). The remaining studies were at low risk of other bias.

\section{Effects of interventions}

See: Summary of findings for the main comparison Acupuncture compared to sham procedure for treating chronic prostatitis/ chronic pelvic pain syndrome; Summary of findings 2 Acupuncture compared to medical treatment for treating chronic prostatitis/chronic pelvic pain syndrome; Summary of findings 3 Circumcision plus usual care compared to waiting list plus usual care for chronic prostatitis/chronic pelvic pain syndrome; Summary of findings 4 Electromagnetic chair compared to control intervention for chronic prostatitis/chronic pelvic pain syndrome; Summary of findings $\mathbf{5}$ Lifestyle modifications compared to control for chronic prostatitis/chronic pelvic pain syndrome; Summary of findings 6 Physical activity compared to control intervention for chronic prostatitis/chronic pelvic pain syndrome; Summary of findings 7 Prostatic massage compared to no intervention for treating chronic prostatitis/chronic pelvic pain syndrome; Summary of findings 8 Extracorporeal shockwave therapy compared to control procedure for chronic prostatitis/ chronic pelvic pain syndrome; Summary of findings 9 Transrectal thermotherapy compared to medical treatment for chronic prostatitis/chronic pelvic pain syndrome; Summary of findings 10 Transrectal thermotherapy (add-on) compared to medical treatment alone for chronic prostatitis/chronic pelvic pain syndrome

\section{Acupuncture}

\section{Acupuncture versus sham procedure}

Three studies with 204 participants compared acupuncture versus sham procedure for short-term follow-up (six to 24 weeks) (Lee 2008; Lee 2009; Sahin 2015). These studies compared the use of acupuncture with a sham procedure in which the acupuncture needles were placed in a point separate from those indicated by the acupuncture technique. One of these studies included the use of electric stimulation (Lee 2009), also called electroacupuncture, in the active treatment group (see Table 1 and Table 2 for further details of the participants and interventions). See Summary of findings table 1 .

\subsection{Prostatitis symptoms}

Three studies with 204 participants reported prostatitis symptoms (Lee 2008; Lee 2009; Sahin 2015). Acupuncture appreciably reduced prostatitis symptoms compared to a sham procedure, measured by $\mathrm{NIH}$-CPSI score at six to eight weeks' follow-up (fixed-effect meta-analysis; MD $-5.79,95 \% \mathrm{Cl}-7.32$ to -4.26 ; Analysis 1.1). These lower scores were observed across all subscores of pain, urinary symptoms and QoL (Analysis 1.2; Analysis 1.3; Analysis 1.4). The quality of evidence was moderate due to unclear risk of bias in one domain (allocation concealment).

Two studies with 113 participants reported the number of participants who achieved an MCID of 6-point decrease of $\mathrm{NIH}$ CPSI score at six weeks, defined as "responders" (Lee 2008; Lee 2009). Acupuncture may have resulted in little to no difference in responder rate compared to a sham procedure (random-effects meta-analysis; RR 2.49, $95 \% \mathrm{Cl} 0.77$ to 8.02 ; Analysis 1.5). The quality of evidence was low due to imprecision issues (small sample size and few events) and inconsistency (statistical heterogeneity 12 $=76 \%$ ).

One of the studies with 91 participants reported the NIH-CPSI scores at the 24 weeks' follow-up (Sahin 2015). Acupuncture reduced prostatitis symptoms in an appreciable number of participants (MD -7.36, 95\% Cl -9.93 to -4.79 ; Analysis 1.6). These lower scores were observed across all subscores of pain, urinary symptoms and QoL (see Analysis 1.7; Analysis 1.8; Analysis 1.9). The quality of evidence was high.

\subsection{Adverse events}

Three studies with 204 participants reported adverse events (Lee 2008; Lee 2009; Sahin 2015). Acupuncture likely resulted in little to no difference in adverse events (RR $1.33,95 \% \mathrm{Cl} 0.51$ to 3.46 ; Analysis 1.10). The quality of evidence was low due to imprecision (small sample size and few events) and unclear risk of bias in one domain (allocation concealment).

\subsection{Sexual dysfunction}

One study with 89 participants reported sexual dysfunction (Lee 2008). Acupuncture likely resulted in little to no difference in sexual dysfunction (fixed-effect meta-analysis; MD $-0.50,95 \% \mathrm{Cl}-3.46$ to 2.46; Analysis 1.11). The quality of evidence was low due to imprecision (wide Cls includes both appreciable benefit and harm) and unclear risk of bias in one domain (allocation concealment). 


\subsection{Urinary symptoms}

Two studies with 113 participants reported urinary symptoms (Lee 2008; Lee 2009). Acupuncture may have resulted in a small effect on urinary symptoms (fixed-effect meta-analysis; MD $-2.79,95 \% \mathrm{Cl}$ -4.77 to -0.82 ; Analysis 1.12 ). The quality of evidence was moderate due to unclear risk of bias in one domain (allocation concealment)..

\subsection{Quality of life}

None of the studies reported QoL.

\subsection{Depression and anxiety}

None of the studies reported depression and anxiety.

\section{Acupuncture versus medical treatment}

Three studies with 245 participants compared acupuncture versus medical treatment (Chen 2009; Kucuk 2015; Lee 2009). These studies compared the use of acupuncture with medical treatment. Medical treatment included the use of pollen extract (Chen 2009), levofloxacin and ibuprofen (Kucuk 2015), or exercise and medical advice (Lee 2009). One of these studies included the use of electric stimulation, also called electroacupuncture, in the active treatment group (Lee 2009; see Table 1 and Table 2 for further details of the participants and interventions). See Summary of findings 2.

\subsection{Prostatitis symptoms}

Three studies reported prostatitis symptoms (Chen 2009; Kucuk 2015; Lee 2009). Acupuncture may have reduced prostatitis symptoms compared to medical treatment, measured by NIH-CPSI score at six to eight weeks' follow-up (MD -4.09, 95\% Cl -6.87 to $-1.30 ; 1^{2}=70 \%$, random-effects meta-analysis) (Analysis 2.1). These lower scores were observed across all subscores of pain, urinary symptoms and QoL (Analysis 2.2; Analysis 2.3; Analysis 2.4). Heterogeneity was predominantly attributable to one study (Chen 2009), which included participants with CP/CPPS using criteria that differed from those recommended by the Research Consensus. In a sensitivity analysis (see below), we excluded the results from this study and we found greater statistical consistency $(12=0 \%)$. Therefore, we chose to report these results in Summary of findings 2. For this reason, we did not downgrade due to inconsistency. The quality of evidence was moderate due to high risk of bias (the studies were not blinded, which affected both detection and performance bias).

One study with 24 participants reported the number of participants who achieved an MCID of 6-point decrease of NIH-CPSI score at six weeks, defined as "responders" (Lee 2009). Acupuncture may have increased the number of responders compared to medical therapy (RR 3.57, $95 \% \mathrm{Cl} 1.45$ to 8.80 ; Analysis 2.5 ). The quality of evidence was low due to high risk of bias (the studies were not blinded, which affected both detection and performance bias) and imprecision (small sample size and few events).

\subsection{Adverse events}

Two studies with 78 participants reported adverse events (Kucuk 2015; Lee 2009). There were no adverse events in either arm (Analysis 2.6). The quality of evidence was low due to high risk of bias (the studies were not blinded, which affected both detection and performance bias) and imprecision (small sample size and no events).

\subsection{Sexual dysfunction}

None of the studies reported sexual dysfunction.

\subsection{Urinary symptoms}

Two studies reported urinary symptoms (Lee 2008; Lee 2009). Acupuncture likely resulted in little to no difference compared to medical therapy, measured by IPSS score at six weeks (MD $-2.70,95 \% \mathrm{Cl}-6.00$ to 0.60 ) (Analysis 2.7 ). The quality of evidence was moderate due to imprecision (wide Cls that included both appreciable benefit and harm).

\subsection{Quality of life}

None of the studies reported QoL.

\subsection{Depression and anxiety}

None of the studies reported depression and anxiety.

\section{Acupuncture versus acupuncture with moxibustion}

One study with 83 participants compared acupuncture versus acupuncture with moxibustion (Chen 2009) (see Table 1; Table 2 for further details of the participants and interventions).

\subsection{Prostatitis symptoms}

The study reported prostatitis symptoms. Moxibustion acupuncture probably reduced prostatitis symptoms compared to regular acupuncture, measured by NIH-CPSI score at eight weeks' follow-up (MD -4.16, 95\% Cl-7.16 to -1.16) (Analysis 3.1). The quality of evidence was moderate due to high risk of bias (the study was not blinded, which affected both detection and performance bias).

\subsection{Adverse events}

The study did not report adverse events.

\subsection{Sexual dysfunction}

The study did not report sexual dysfunction.

\subsection{Urinary symptoms}

The study did not report urinary symptoms.

\subsection{Quality of life}

The study did not report QoL.

\subsection{Depression and anxiety}

The study did not report depression and anxiety.

\section{Circumcision: early versus delayed circumcision}

One study with 713 participants compared early versus delayed circumcision (Zhao 2015). This study compared the effects of assigning participants to circumcision at four weeks compared with a waiting list to be circumcised at a delayed interval of three months (see Table 1 and Table 2 for further details of the participants and interventions). See Summary of findings 3.

\subsection{Prostatitis symptoms}

Circumcision probably reduced prostatitis symptoms compared to delayed circumcision, measured by NIH-CPSI score at 12 weeks' follow-up (MD -3.00, 95\% Cl -3.82 to -2.18) (Analysis 4.1). These lower scores were observed across all subscores of pain, urinary symptoms and QoL (Analysis 4.2; Analysis 4.3; Analysis 4.4). The 
quality of evidence was moderate due to high risk of bias (the study was not blinded, which affected both detection and performance bias).

\subsection{Adverse events}

Circumcision likely resulted in little to no difference in adverse events compared to delayed circumcision group (RR $1.23,95 \% \mathrm{Cl}$ 0.86 to 1.76 ) (Analysis 4.5). The quality of evidence was low due to high risk of bias (the study was not blinded, which affected both detection and performance bias) and imprecision (small sample size and few events).

\subsection{Sexual dysfunction}

The study did not report sexual dysfunction.

\subsection{Urinary symptoms}

The study did not report urinary symptoms.

\subsection{Quality of life}

The study did not report QoL.

\subsection{Depression and anxiety}

The study did not report depression and anxiety.

\section{Electromagnetic chair versus control intervention (inactive device)}

Two studies with 57 participants compared electromagnetic chair versus control intervention (Paick 2006; Rowe 2005). The control intervention was either the electromagnetic chair that was switched off or medical therapy (see Table 1; Table 2 for further details of the participants and interventions). See Summary of findings 4 .

\subsection{Prostatitis symptoms}

Both studies reported prostatitis symptoms. The study by Paick 2006 reported that the active treatment group had similar symptoms to the control group, measured by NIH-CPSI score at six weeks' follow-up (MD -3.00, 95\% Cl-7.75 to 1.75) (Analysis 5.1). This was also observed across all subscores of pain, urinary symptoms and QoL (Analysis 5.2; Analysis 5.3; Analysis 5.4). The study by Rowe 2005 found that the active treatment group had fewer symptoms (mean score 26.4) compared to the control group (mean score 42.4) at 12 weeks, measured by a validated 0 to 90 scale of symptoms for prostatitis. This study did not report information for effect size calculation or P value. This study also reported results at one year' follow-up: the mean score for the active treatment group was 24 and the mean score in the control group was 33.6. Therefore, we were uncertain about the effects of the electromagnetic chair on prostatitis symptoms. The quality of evidence was very low due to high risk of bias (the studies were not blinded, which affected both detection and performance bias), attrition bias, selective reporting of outcomes for the Rowe 2005 study and small sample size in each study that resulted in imprecision and wide Cls. Additionally, there was inconsistency in the findings. Meta-analysis of these studies was not possible due to missing information regarding standard deviations in Rowe 2005.

\subsection{Adverse events}

Electromagnetic chair likely resulted in little to no difference in adverse events compared to the control intervention (RR 2.18,
$95 \% \mathrm{Cl} 0.10$ to 46.92 ) (Analysis 5.5). The quality of evidence was low due to high risk of bias (the studies were not blinded, which affected both detection and performance bias) and imprecision (small sample size and few events).

\subsection{Sexual dysfunction}

The studies did not report sexual dysfunction.

\subsection{Urinary symptoms}

The study by Paick 2006 indicated that the electromagnetic chair may have resulted in no difference in urinary symptoms compared to the control group, measured by IPSS score at six weeks' follow-up (MD $0.00,95 \% \mathrm{Cl}-4.13$ to 4.13 ) (Analysis 5.6). The quality of evidence was low due to high risk of bias (the study was not blinded, which affected both detection and performance bias) and imprecision (small sample size and few events).

\subsection{Quality of life}

The studies did not report QoL.

\subsection{Depression and anxiety}

The studies did not report depression and anxiety.

\section{Lifestyle modifications versus control (no intervention)}

One study with 100 participants compared lifestyle modifications versus control (no intervention) (Gallo 2014). This study compared the effects of instructing participants to change some aspects in their lifestyle related to risk factors for CP/CPPS to no intervention, that is, maintaining the same lifestyle (see Table 1 and Table 2 for further details of the participants and interventions). See Summary of findings 5.

\subsection{Prostatitis symptoms}

The study analysed the number of participants who achieved a 6point decrease in NIH-CPSI scores at three months ("responders"). Lifestyle modifications may have increased the number of responders in terms of prostatitis symptoms, but we were very uncertain (RR 3.90, 95\% $\mathrm{Cl} 2.20$ to 6.92) (Analysis 6.1). The quality of evidence was very low due to high risk of selection bias (unconcealed allocation), detection and performance bias (the study was not blinded), missing outcome data and suspected selective outcome reporting (NIH-CPSI scores were only presented graphically). Additionally, there were few response events in each group, resulting in imprecision.

\subsection{Adverse events}

The study did not report adverse events.

\subsection{Sexual dysfunction}

The study did not report sexual dysfunction.

\subsection{Urinary symptoms}

The study did not report urinary symptoms.

\subsection{Quality of life}

The study did not report QoL.

\subsection{Depression and anxiety}

The study did not report depression and anxiety. 


\section{Physical activity versus control intervention}

One study with 85 participants compared physical activity versus control intervention (Giubilei 2007). This study compared the effects of a regular exercise programme to a control intervention (see Table 1 and Table 2 for further details of the participants and interventions). See Summary of findings 6 .

\subsection{Prostatitis symptoms}

Physical activity may have reduced prostatitis symptoms, measured by NIH-CPSI score at six weeks' follow-up, but we were very uncertain (MD $-2.50,95 \% \mathrm{Cl}-4.69$ to -0.31 ) (Analysis 7.1 ). These lower scores were observed across the subscores of pain and QoL (Analysis 7.2; Analysis 7.4); however, participants in the intervention group had higher urinary symptoms (Analysis 7.3). The quality of evidence was low due to high risk of performance bias and detection bias (the study was not blinded) and high risk of attrition bias.

\subsection{Adverse events}

The study did not report adverse events.

\subsection{Sexual dysfunction}

The study did not report sexual dysfunction.

\subsection{Urinary symptoms}

The study did not report urinary symptoms.

\subsection{Quality of life}

The study did not report QoL.

\subsection{Depression and anxiety}

The study measured symptoms of anxiety with the SAI-Y score and symptoms of depression with the Beck Depression Inventory at six weeks' follow-up. The scores for anxiety and depression were similar in each group (Analysis 7.5; Analysis 7.6). The quality of evidence was very low due to high risk of performance bias and detection bias (the study was not blinded), high risk of attrition bias and imprecision issues.

\section{Prostatic massage versus no intervention}

Two studies with 115 participants compared prostatic massage versus no intervention (Ateya 2006; Shen 2006). In Shen 2006, all participants were also treated with traditional Chinese medicine as cointervention (see Table 1 and Table 2 for further details of the participants and interventions). See Summary of findings 7.

\subsection{Prostatitis symptoms}

Both studies reported this outcome, however only Ateya 2006 reported the total NIH-CPSI scores. In this study, prostatic massage may not have reduced prostatitis symptoms compared to no intervention, measured by NIH-CPSI score at four weeks' follow-up (MD -1.10, 95\% Cl -5.63 to 3.43) (Analysis 8.1). These similar scores were observed across the subscores of pain, urinary symptoms and QoL (Analysis 8.2; Analysis 8.3; Analysis 8.4). The study by Shen 2006 only reported the subscores, and participants who were assigned to prostatic massage had lower subscores for pain, urinary symptoms and QoL compared to those who did not received the intervention. The quality of evidence was very low due to high risk of performance and detection bias (study not blinded), unclear risk of bias in all the remaining domains and imprecision.

\subsection{Adverse events}

The studies did not report adverse events.

\subsection{Sexual dysfunction}

The studies did not report sexual dysfunction.

\subsection{Urinary symptoms}

The studies did not report urinary symptoms.

\subsection{Quality of life}

The studies did not report QoL.

\subsection{Depression and anxiety}

The studies did not report depression and anxiety.

\section{Extracorporeal shockwave therapy versus control}

Four studies with 237 participants compared extracorporeal shockwave therapy (ESWT) versus control (Pajovic 2016; Vahdatpour 2013; Zeng 2012; Zimmermann 2009). In three studies this included a form of blinding using a sham procedure in which the device was turned off (Vahdatpour 2013; Zeng 2012; Zimmermann 2009). In one studies, the control group did not receive any form of ESWT (Pajovic 2016; see Table 1 and Table 2 for further details of the participants and interventions). See Summary of findings 8 .

\subsection{Prostatitis symptoms}

Three studies with 157 participants reported the NIH-CPSI scores for prostatitis symptoms (Pajovic 2016; Vahdatpour 2013; Zimmermann 2009). We found that ESWT reduced prostatitis symptoms compared to the control intervention, measured by $\mathrm{NIH}-\mathrm{CPSI}$ score at 12 weeks' follow-up (MD $-6.18,95 \% \mathrm{Cl}-7.46$ to $-4.89 ; 1^{2}=34 \%$; random-effects meta-analysis) (Analysis 9.1). These lower scores were observed across the subscores of pain, urinary symptoms and QoL (Analysis 9.2; Analysis 9.3; Analysis 9.4). Since one of the studies was not blinded (Pajovic 2016), the evidence could have been downgraded for high risk of detection and performance bias; however, the results of this study were consistent with those with low risk of bias, therefore we did not downgrade the quality of evidence (high-quality evidence).

Two studies with 135 participants reported prostatitis symptoms analysing the number of participants who achieved a 6-point decrease in NIH-CPSI scores at 12 weeks' follow-up (Zeng 2012; Zimmermann 2009). ESWT may have had little to no effect in the number of responders compared to the control intervention (RR $6.20,95 \% \mathrm{Cl} 0.48$ to $79.79 ; \mathrm{I}^{2}=71 \%$; random-effects meta-analysis) (Analysis 9.5). The quality of evidence was very low due to high risk of detection and performance bias (one study was not blinded), inconsistency and imprecision issues.

Two studies with 97 participants reported prostatitis symptoms at 24 weeks' follow-up (Pajovic 2016; Vahdatpour 2013). ESWT may have had little to no effect on prostatitis symptoms compared to the control intervention (MD $-2.23,95 \% \mathrm{Cl}-5.98$ to $1.53 ; \mathrm{I}^{2}=82 \%$; random-effects meta-analysis) (Analysis 9.6). These similar scores were observed across the subscores of pain, urinary symptoms and 
QoL (Analysis 9.7; Analysis 9.8; Analysis 9.9). The quality of evidence was low due to high risk of performance and detection bias (one study was not blinded) and inconsistency.

\subsection{Adverse events}

Three studies with 195 participants reported adverse events (Pajovic 2016; Zeng 2012; Zimmermann 2009). Two studies reported no adverse events in either group (Zeng 2012; Zimmermann 2009). The numbers of participants who had adverse events were similar in the ESWT and control group in the third study (Pajovic 2016) (RR $1.22,95 \% \mathrm{Cl} 0.59$ to 2.51 ) (Analysis 9.10). The quality of evidence was low due to high risk of performance and detection bias (one study was not blinded) and imprecision issues.

\subsection{Sexual dysfunction}

One study with 60 participants reported sexual dysfunction (Zimmermann 2009). ESWT probably reduced sexual dysfunction compared to control, measured by the IIEF scale at 12 weeks (MD $3.34,95 \% \mathrm{Cl} 2.68$ to 4.00 ) (Analysis 9.11). For the IIEF scale, higher scores indicated fewer symptoms. The quality of evidence was moderate due to imprecision issues.

\subsection{Urinary symptoms}

One study with 60 participants reported urinary symptoms (Zimmermann 2009). ESWT probably reduced urinary symptoms compared to control, measured by the IIEF scale at 12 weeks (MD $-4.50,95 \% \mathrm{Cl}-5.14$ to -3.86 ) (Analysis 9.12). The quality of evidence was moderate due to imprecision issues.

\subsection{Quality of life}

The studies did not report QoL.

\subsection{Depression and anxiety}

The studies did not report depression and anxiety.

\section{Transrectal thermotherapy versus medical therapy or as add-on to medical therapy}

Two studies with 237 participants compared transrectal thermotherapy (TRT) versus medical therapy or as add-on to medical therapy (Gao 2012; Yoo 2009). In both studies, participants were randomised to one of the following groups: medical therapy, TRT or the combination of medical therapy and TRT. Four additional studies with 200 participants evaluated the effects of TRT (Muraro 1995; Oh 2009; Shah 1993; Vassily 1999), however there were no relevant outcome data available for this review. One study with 57 participants evaluated the effects of thermotherapy in three different regimens: one session weekly for four weeks, one session weekly for six weeks and two sessions weekly for three weeks (Montorsi 1993; see Table 1 and Table 2 for further details of the participants and interventions). See Summary of findings 9 and Summary of findings 10 .

\subsection{Prostatitis symptoms}

\subsubsection{Transrectal thermotherapy versus medical therapy}

Two studies compared the effect of TRT versus medical therapy on prostatitis symptoms. TRT may have decreased prostatitis symptoms compared to medical therapy, measured by NIH-CPSI score at six to 12 weeks' follow-up (MD $-2.50,95 \% \mathrm{Cl}-3.82$ to $-1.18 ; 1^{2}=0 \%$, fixed-effect meta-analysis) (Analysis 10.1). Only Yoo 2009 reported the results of the subscores; these lower scores were observed for urinary symptoms and QoL, but not in the pain domain (Analysis 10.2; Analysis 10.3; Analysis 10.4). The quality of evidence was low due to high risk of allocation concealment bias, performance and detection bias (the study was not blinded) and high risk of attrition bias.

\subsubsection{Transrectal thermotherapy plus medical therapy versus medical therapy alone}

Both studies compared the effect of TRT plus medical therapy versus medical therapy alone on prostatitis symptoms. TRT plus medical therapy may have decreased prostatitis symptoms compared to medical therapy alone, measured by NIH-CPSI score at six to 12 weeks' follow-up (MD $-4.34,95 \% \mathrm{Cl}-5.65$ to $-3.04 ; 12=0 \%$, fixed-effect meta-analysis) (Analysis 10.1). Only Yoo 2009 reported the results of the subscores, these lower scores were observed across the subscores of pain, urinary symptoms and QoL (Analysis 10.2; Analysis 10.3; Analysis 10.4). The quality of evidence was low due to high risk of allocation concealment bias, performance and detection bias (the study was not blinded), and high risk of attrition bias.

\subsection{Adverse events}

Montorsi 1993 did not compare the intervention to a control group but compared different regimens. It reported that none of the participants had from "major complications." The other studies did not report adverse effects. Yoo 2009 reported that participants with active treatment had itching and tenesmus, but it was not quantified.

\subsection{Sexual dysfunction}

The studies did not report sexual dysfunction.

\subsection{Urinary symptoms}

The studies did not report urinary symptoms.

\subsection{Quality of life}

The studies did not report QoL.

\subsection{Depression and anxiety}

The studies did not report depression and anxiety.

\section{Biofeedback}

One study with 140 participants used biofeedback (Yang 2011). This factorial study had four groups: biofeedback added to usual care (40 participants), biofeedback with electric stimulation added to usual care (40 participants), electrical stimulation added to usual care (40 participants) and usual care alone (20 participants). Usual care included a series of lifestyle modifications (see Table 1; Table 2).

\subsection{Prostatitis symptoms}

\subsubsection{Biofeedback versus usual care}

Biofeedback may have decreased prostatitis symptoms compared to usual care, measured by NIH-CPSI scores one month after treatment (MD -10.42, $95 \% \mathrm{Cl}-11.93$ to -8.91). These lower scores were observed across all subscores of pain, urinary symptoms and QoL (Analysis 11.2; Analysis 11.3; Analysis 11.4). The quality of evidence was low due to high risk of performance and detection bias (the study was not blinded) and unclear risk of bias in most of the remaining domains. 


\subsubsection{Electrical stimulation versus usual care}

Electrical stimulation may have decreased prostatitis symptoms compared to usual care, measured by $\mathrm{NIH}$-CPSI scores at one month after treatment (MD $-10.63,95 \% \mathrm{Cl}-12.13$ to -9.13$)$. These lower scores were observed across all subscores of pain, urinary symptoms and QoL domains (Analysis 11.2; Analysis 11.3; Analysis 11.4). The quality of evidence was low due to high risk of performance and detection bias (the study was not blinded) and unclear risk of bias in most of the remaining domains.

\subsubsection{Biofeedback plus electrical stimulation versus usual care}

Biofeedback plus electric stimulation may have decreased prostatitis symptoms compared to usual care, measured by $\mathrm{NIH}-$ CPSI scores at one month after treatment (MD $-15.83,95 \% \mathrm{Cl}-17.72$ to -13.94). These lower scores were observed across all subscores of pain, urinary symptoms and QoL domains (Analysis 11.2; Analysis 11.3; Analysis 11.4). The quality of evidence was low due to high risk of performance and detection bias (the study was not blinded) and unclear risk of bias in most of the remaining domains.

\subsection{Adverse events}

The study reported that no participants experienced adverse events. The quality of evidence was very low due to high risk of performance and detection bias (the study was not blinded) and unclear risk of bias in most of the remaining domains and imprecision (few events).

\subsection{Sexual dysfunction}

The study did not report sexual dysfunction.

\subsection{Urinary symptoms}

The study did not report urinary symptoms.

\subsection{Quality of life}

The study did not report QoL.

\subsection{Depression and anxiety}

The study did not report depression and anxiety.

\section{External radiofrequency hyperthermia with or without terazosin}

One study with 136 participants compared external radiofrequency hyperthermia $(\mathrm{ERH})$ with terazosin versus ERH without terazosin (Wang 2002) (see Table 1; Table 2).

\subsection{Prostatitis symptoms}

ERH with terazosin may have decreased prostatitis symptoms (validated 0 to 12 score) compared to ERH alone at 12 weeks (MD $-2.00,95 \% \mathrm{Cl}-2.48$ to -1.52 ) (Analysis 12.1 ). The quality of evidence was low due to high risk of performance and detection bias (the study was not blinded) and unclear risk of bias in most of the remaining domains.

\subsection{Adverse events}

Two participants had dizziness in the group who received the combination of ERH and terazosin in comparison to none in the ERH alone group (RR 5.91, 95\% Cl 0.29 to 121.23 ) (Analysis 12.2). The quality of evidence was very low due to high risk of performance and detection bias (the study was not blinded) and unclear risk of bias in most of the remaining domains and imprecision (few events).

\subsection{Sexual dysfunction}

The study did not report sexual dysfunction.

\subsection{Urinary symptoms}

The study did not report urinary symptoms.

\subsection{Quality of life}

The study did not report QoL.

\subsection{Depression and anxiety}

The study did not report depression and anxiety.

\section{Laser therapy applied to the prostate compared with medical care}

One study with 112 participants compared laser therapy with medical care (Fang 2005) (see Table 1; Table 2).

\subsection{Prostatitis symptoms}

Clinical response was defined as a decrease of $60 \%$ or more in prostatitis symptoms assessed with $\mathrm{NIH}-\mathrm{CPSI}$ at six weeks. Laser therapy may have increased the response rate compared to medical treatment, but we were very uncertain (RR $2.35,95 \% \mathrm{Cl} 1.53$ to 3.62 ) (Analysis 13.1). The quality of evidence was very low due to high risk of performance and detection bias (the study was not blinded) and unclear risk of bias in most of the remaining domains and imprecision (few events).

\subsection{Adverse events}

None of the participants in the study had adverse events (Analysis 13.2). The quality of evidence was very low due to high risk of performance and detection bias (the study was not blinded) and unclear risk of bias in most of the remaining domains and imprecision (zero events).

\subsection{Sexual dysfunction}

The study did not report sexual dysfunction.

\subsection{Urinary symptoms}

The study did not report urinary symptoms.

\subsection{Quality of life}

The study did not report QoL.

\subsection{Depression and anxiety}

The study did not report depression and anxiety.

\section{Tibial nerve stimulation compared with no intervention}

One study with 89 participants compared tibial nerve stimulation with no intervention (Kabay 2009). This study compared participants who received tibial nerve stimulation compared to no active intervention (see Table 1; Table 2).

\subsection{Prostatitis symptoms}

Tibial nerve stimulation probably reduced prostatitis symptoms measured by NIH-CPSI compared to no active treatment at 12 weeks (MD -11.20, 95\% Cl -12.92 to -9.48) (Analysis 14.1). These 
lower scores were observed across all subscores of pain, urinary symptoms and QoL (Analysis 14.2; Analysis 14.3; Analysis 14.4). The quality of evidence was moderate due to high risk of performance and detection bias.

\subsection{Adverse events}

The study did not report adverse events.

\subsection{Sexual dysfunction}

The study did not report sexual dysfunction.

\subsection{Urinary symptoms}

The study did not report urinary symptoms.

\subsection{Quality of life}

The study did not report QoL.

\subsection{Depression and anxiety}

The study did not report depression and anxiety.

\section{Sono-electromagnetic therapy versus placebo intervention}

One study with 60 participants compared sono-electromagnetic therapy versus placebo intervention (Kessler 2014). This study had two groups: participants who were treated at home with a sonoelectromagnetic therapy device and participants treated with a 'placebo' device (see Table 1; Table 2).

\subsection{Prostatitis symptoms}

Sono-electromagnetic therapy probably resulted in little to no effect on prostatitis symptoms compared to the placebo device at 16 weeks (MD -2.80, 95\% $\mathrm{Cl}-6.75$ to 1.15 ). These similar scores were observed across all subscores of pain, urinary symptoms and QoL domains (Analysis 17.3; Analysis 17.4; Analysis 17.5). There was a similar number of 'responders' (defined as a drop in 4 points of $\mathrm{NIH}$ CPSI score) in each group (RR $1.40,95 \% \mathrm{Cl} 0.91$ to 2.15 ). The quality of evidence was moderate due to imprecision issues.

The study authors presented subgroup analysis.

- Participants aged less than 50 and 50 years or older had similar reductions in NIH-CPSI scores $(P=0.40)$.

- Participants with baseline NIH-CPSI scores greater than 25 and 25 or less had similar reductions in NIH-CPSI scores $(P=0.35)$.

- Participants with a duration of symptoms greater than 12 months had a lower reduction of NIH-CPSI scores compared to participants who had a shorter duration of symptoms $(P=0.023)$. The MDs in NIH-CPSI score were $-0.8(95 \% \mathrm{Cl}-4.6$ to 3.1$)$ with a duration of symptoms greater than 12 months and $-8.5(95 \% \mathrm{Cl}$ -14.3 to -2.6 ) with a shorter duration of symptoms.

\subsection{Adverse events}

One participant $(1 / 30)$ in the active treatment group had worsening of pain symptoms. There were no adverse events observed in the control group. The quality of evidence was low due to imprecision issues (few events).

\subsection{Sexual dysfunction}

The study did not report sexual dysfunction.

\subsection{Urinary symptoms}

The study did not report urinary symptoms.

\subsection{Quality of life}

The study did not report QoL.

\subsection{Depression and anxiety}

The study did not report depression and anxiety.

\section{Myofascial trigger point release therapy compared to control intervention (massage)}

One study with 23 men compared myofascial trigger point release therapy with control intervention (massage) (Fitzgerald 2013). This study also included women with interstitial cystitis/painful bladder syndrome, but their results were not included in this review. This study had two groups: participants who were treated with myofascial physical therapy and participants who received therapeutic western massage as a control intervention (see Table 1; Table 2).

\subsection{Prostatitis symptoms}

Myofascial trigger point release therapy may have resulted in little to no effect compared to the control intervention at 12 weeks (MD $1.00,95 \% \mathrm{Cl}-6.45$ to 8.45 ). These similar scores were observed across all subscores of pain, urinary symptoms and QoL (Analysis 15.2; Analysis 15.3; Analysis 15.4). The quality of evidence was very low due to high risk of performance and detection bias (the study was not blinded), the presence of baseline differences and imprecision issues (wide Cls due to small sample size).

\subsection{Adverse events}

The authors reported the incidence of adverse events globally for men and women. Even though we contacted study authors to obtain the disaggregated data for men with CP/CPPS, we received no additional information.

\subsection{Sexual dysfunction}

Myofascial trigger point release may have resulted in little to no effect compared to the control intervention, measured by the Sexual Health Inventory for Men (MD $-2.20,95 \% \mathrm{Cl}-9.24$ to 4.84 ) (Analysis 15.5). The quality of evidence was very low due to high risk of performance and detection bias (the study was not blinded), the presence of baseline differences and imprecision issues (wide confidence interval due to small sample size).

\subsection{Urinary symptoms}

The study did not report urinary symptoms.

\subsection{Quality of life}

Myofascial trigger point release therapy may have resulted in little to no effect on QoL compared to the control intervention, measured by the SF-12 Health Status Questionnaire (MD $-1.30,95 \% \mathrm{Cl}-9.54$ to 6.94 for the physical domain and $0.80,95 \% \mathrm{Cl}-9.25$ to 10.85 for the mental domain) (Analysis 15.6; Analysis 15.7). The quality of evidence was very low due to high risk of performance and detection bias (the study was not blinded), the presence of baseline differences and imprecision issues (wide $\mathrm{Cls}$ due to small sample size). 


\subsection{Depression and anxiety}

The study did not report depression and anxiety.

\section{Osteopathy versus control intervention (exercise programme)}

One study with 35 participants compared osteopathy versus control intervention (exercise programme) (Marx 2009) (see Table 1; Table 2).

\subsection{Prostatitis symptoms}

Osteopathy may have reduced prostatitis symptoms, measured by the NIH-CPSI score, compared the control intervention at 14 weeks (MD -9.67, 95\% Cl-15.15 to -4.19) (Analysis 16.1). The authors also reported a decrease in QoL subscore (Analysis 16.2), but did not report the other subscores. The quality of evidence was very low due to high risk of performance and detection bias (the study was not blinded), the unbalanced attrition at follow-up and imprecision issues (wide $\mathrm{Cl}$ due to small sample size).

\subsection{Adverse events}

The study did not report adverse events.

\subsection{Sexual dysfunction}

The study did not report sexual dysfunction.

\subsection{Urinary symptoms}

Osteopathy may reduce urinary symptoms, measured by IPSS score, compared to the control intervention (MD -8.70, 95\% Cl -12.73 to -4.67$)$. The quality of evidence was very low due to high risk of performance and detection bias (the study was not blinded), the unbalanced attrition at follow-up and imprecision issues (wide Cls due to small sample size).

\subsection{Quality of life}

The study did not report QoL.

\subsection{Depression and anxiety}

The study did not report depression and anxiety.

\section{Transcutaneous electrical nerve stimulation compared to control intervention}

Two studies with 56 participants compared TENS with control (Samhan 2011; Sikiru 2008). In Samhan 2011, the control group received a sham procedure in which the TENS device was switched off, and, in Sikiru 2008, the control group received no intervention (see Table 1; Table 2).

\subsection{Prostatitis symptoms}

The studies did not report the total NIH-CPSI scores. They reported the pain subscore at four weeks. TENS may have appreciably reduced prostatitis-related pain compared to the control intervention, measured by the NIH-CPSI pain subscore (MD $-8.60,95 \% \mathrm{Cl}-9.71$ to $-7.48 ; \mathrm{I}^{2}=97 \%$; random-effects meta-analysis) (Analysis 18.1). The quality of evidence was very low due to high risk of performance and detection bias (one study was not blinded), inconsistency and imprecision.

\subsection{Adverse events}

\subsection{Sexual dysfunction}

The studies did not report sexual dysfunction.

\subsection{Urinary symptoms}

The studies did not report urinary symptoms.

\subsection{Quality of life}

The studies did not report QoL.

\subsection{Depression and anxiety}

The studies did not report depression and anxiety.

\section{Transurethral thermotherapy compared to control intervention}

Two studies with 62 participants compared transurethral thermotherapy with control intervention (Kastner 2004; Nickel 1996). These studies assessed transurethral thermotherapy at approximately $50{ }^{\circ} \mathrm{C}$ compared to a sham procedure with no temperature elevation (Nickel 1996), or to a transurethral thermotherapy at $70{ }^{\circ} \mathrm{C}$ (Kastner 2004) (see Table 1; Table 2).

\subsection{Prostatitis symptoms}

\subsubsection{Transurethral thermotherapy at $50^{\circ} \mathrm{C}$ compared to sham procedure}

Nickel 1996 recruited 20 participants and reported that participants who received transurethral thermotherapy had fewer symptoms of prostatitis compared to participants who received the sham procedure with no temperature elevation at three months' followup, using the Prostatitis Symptom Severity Index (range 0 to 100) with a mean score of 27.3 with transurethral thermotherapy and 52.9 with sham $(P<0.05)$. The quality of evidence was low due to severe imprecision.

\subsubsection{Transurethral thermotherapy at $55^{\circ} \mathrm{C}$ compared to thermotherapy at $70^{\circ} \mathrm{C}$}

Kastner 2004 recruited 42 participants and reported that participants who received transurethral thermotherapy at $70{ }^{\circ} \mathrm{C}$ had similar symptoms of prostatitis compared to participants who received the procedure at $55{ }^{\circ} \mathrm{C}$ at three months' follow-up, using the NIH-CPSI score (MD -1.10, $95 \% \mathrm{Cl}-6.50$ to 4.30) (Analysis 19.1). These similar scores were observed across all subscores of pain, urinary symptoms and QoL (Analysis 19.2; Analysis 19.3; Analysis 19.4). The quality of evidence was low due to lost to follow-up (attrition bias) and imprecision.

\subsection{Adverse events}

Nickel 1996 recruited 20 participants and specified that four participants experienced transient adverse reactions, but there were no specifications whether they were in the active treatment group or the sham intervention group. Kastner 2004 reported that both active treatment $\left(55^{\circ} \mathrm{C}\right.$ and $\left.70{ }^{\circ} \mathrm{C}\right)$ groups had genitourinary events that resolved at six weeks, and that the proportion of events was similar across groups. The quality of evidence was very low due to high risk of performance, detection and attrition bias, and imprecision.

\subsection{Sexual dysfunction}

The studies did not report sexual dysfunction.

The studies did not report adverse events. 


\subsection{Urinary symptoms}

\subsubsection{Transurethral thermotherapy at $50^{\circ} \mathrm{C}$ compared to sham procedure}

Nickel 1996 recruited 20 participants and reported that participants who received transurethral thermotherapy had fewer urinary symptoms compared to participants who received the sham procedure with no temperature elevation at three months' followup, using the American Urology Association Symptom Score (range 0 to 100) with mean scores of 12.8 with transurethral thermotherapy and 21.9 with sham ( $P$ value not available).

\subsubsection{Transurethral thermotherapy at $55^{\circ} \mathrm{C}$ compared to thermotherapy at $70^{\circ} \mathrm{C}$}

Kastner 2004 recruited 42 participants and reported that participants who received transurethral thermotherapy at $70^{\circ} \mathrm{C}$ had similar urinary symptoms compared to participants who received the procedure at $55^{\circ} \mathrm{C}$ at three months' follow-up (MD - $2.10,95 \% \mathrm{Cl}$ -6.34 to 2.14 ) (Analysis 19.5). The quality of evidence was low due to lost to follow-up (attrition bias) and imprecision.

\subsection{Quality of life}

The studies did not report QoL.

\subsection{Depression and anxiety}

The studies did not report depression and anxiety.

\section{Transurethral needle ablation compared to sham procedure}

One study with 33 participants compared transurethral needle ablation (TUNA) with sham procedure (Leskinen 2002). The sham procedure used urethroscopy but not ablation (see Table 1; Table 2).

\subsection{Prostatitis symptoms}

TUNA may have had little to no effect on prostatitis symptoms compared to a sham procedure at 12 months' follow-up, using the Prostatitis Symptom Severity Index (range 0 to 100) (MD 2.30, 95\% $\mathrm{Cl}-8.02$ to 12.62 ) (Analysis 20.1). The quality of evidence was very low due to severe imprecision and unclear risk of bias in almost all domains.

\subsection{Adverse events}

The study reported that $10 / 25$ participants in the TUNA group and $3 / 8$ in the sham group experienced dysuria during the first month after the procedure and $3 / 25$ participants in the TUNA group reported transient haematuria. The quality of evidence was very low due to severe imprecision and unclear risk of bias in almost all domains.

\subsection{Sexual dysfunction}

The study did not report sexual dysfunction.

\subsection{Urinary symptoms}

TUNA may have had little to no effect on urinary symptoms compared to a sham procedure at 12 months follow-up, using the IPSS score (MD 0.40, 95\% Cl -5.09 to 5.89) (Analysis 20.2). The quality of evidence was very low due to severe imprecision and unclear risk of bias in almost all domains.

\subsection{Quality of life}

The study did not report QoL.

\subsection{Depression and anxiety}

The study did not report depression and anxiety.

\section{Ultrasound compared to or as add-on to medical therapy}

One study with 105 participants had three groups that compared non-intrusive ultrasound alone with Chinese-Western medicine or non-intrusive ultrasound plus integrated Chinese-Western medicine (Kaikai 2014) (see Table 1; Table 2).

\subsection{Prostatitis symptoms}

\subsubsection{Ultrasound versus medical therapy}

Ultrasound therapy may have increased prostatitis symptoms compared to medical therapy, measured by NIH-CPSI scores at one month after treatment (MD 1.09, 95\% Cl 0.16 to 2.02) (Analysis 21.1). These greater scores were observed across all subscores of pain, urinary symptoms and QoL (Analysis 21.2; Analysis 21.3; Analysis 21.4). The quality of evidence was low due to high risk of performance and detection bias (study not blinded), and imprecision.

\subsubsection{Ultrasound plus medical therapy versus medical therapy alone}

Ultrasound plus medical therapy may have resulted in fewer prostatitis symptoms compared to medical therapy alone, measured by NIH-CPSI score at one month after treatment (MD $-6.67,95 \% \mathrm{Cl}-7.62$ to -5.72 ) (Analysis 21.1). These lower scores were observed across all subscores of pain, urinary symptoms and QoL (Analysis 21.2; Analysis 21.3; Analysis 21.4). The quality of evidence was low due to high risk of performance and detection bias (study not blinded), and imprecision.

\subsection{Adverse events}

The study reported five cases of vertigo, six cases of gastrointestinal discomfort and three cases of sleepiness; however, it did not specify which group experienced them. The quality of evidence was low due to high risk of performance and detection bias (study not blinded), and imprecision.

\subsection{Sexual dysfunction}

The study did not report sexual dysfunction.

\subsection{Urinary symptoms}

The study did not report urinary symptoms.

\subsection{Quality of life}

The study did not report QoL.

\subsection{Depression and anxiety}

The study did not report depression and anxiety.

\section{Hypercapnic hypoxia versus no additional intervention}

One study with 37 participants compared hypercapnic hypoxia plus medical therapy versus medical therapy alone (Neimark 2016) (see Table 1; Table 2). 


\subsection{Prostatitis symptoms}

The study did not report prostatitis symptoms.

\subsection{Adverse events}

The study did not report adverse events.

\subsection{Sexual dysfunction}

The study did not report sexual dysfunction.

\subsection{Urinary symptoms}

The study reported that at the end of the 10 days of treatment, the mean IPSS score for the hypercapnic hypoxia plus medical therapy group was 9 and the medical therapy alone was 8 ( $P$ value not available). The quality of evidence was very low due to high risk of detection and performance bias, and imprecision.

\subsection{Quality of life}

The study did not report QoL.

\subsection{Depression and anxiety}

The study did not report depression and anxiety.

\section{TaiJiQuan/t'ai chi ch'uan (\#\#\#) plus usual care versus usual care alone}

One study with 96 participants compared TaiJiQuan/t'ai chi ch'uan (太極拳) plus usual care with usual care alone (Zhang 2011a) (see Table 1; Table 2). This study was poorly reported in the methods and results section. None of the predefined outcomes of this review were reported. The study authors reported different levels of clinical "response" (definition not available).

\section{Psychological support}

We found no studies reporting psychological support.

\section{Prostatic surgery}

We found no studies reporting prostatic surgery.

\section{Sensitivity analysis}

We performed a sensitivity analysis excluding the six studies (Chen 2009; Montorsi 1993; Muraro 1995; Nickel 1996; Shah 1993; Vassily 1999) that did not meet the Research Consensus definition for CP/ CPPS (Nickel 1999a).

\section{Acupuncture}

The study by Chen 2009 was involved in two comparisons.

\subsection{Acupuncture versus medical therapy, prostatitis symptoms}

The sensitivity analysis excluding Chen 2009 resulted in a reduction of the statistical heterogeneity across the studies (Figure 4). The meta-analysis of the two remaining studies showed that acupuncture probably reduced appreciably prostatitis symptoms compared with medical therapy (MD $-6.05,95 \% \mathrm{Cl}-7.87$ to -4.24 ; 78 participants; 4 studies; $1^{2}=0 \%$ ) (Kucuk 2015; Lee 2009). The results of this sensitivity analysis were incorporated in Summary of findings 2 and the other sections of this review.

Figure 4. Forest plot of comparison: 22 Acupuncture treatments versus medical treatment. Sensitivity analysis, outcome: 22.1 Prostatitis symptoms (NIH-CPSI total).

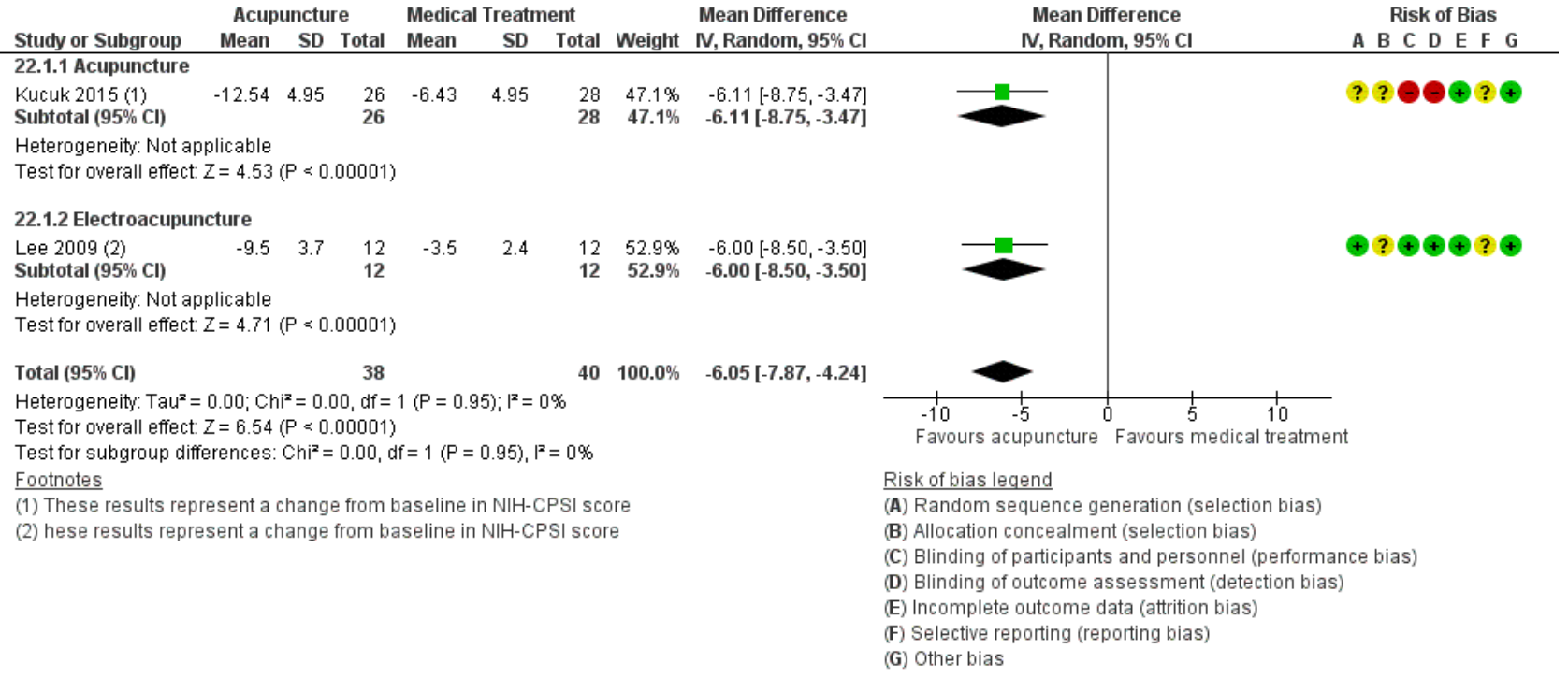

\subsection{Acupuncture versus acupuncture plus moxibustion}

The exclusion of this study resulted in no evidence for this comparison (Chen 2009).

\section{Transrectal thermotherapy}

The exclusion of three of the studies that did not meet the Research Consensus definition did not affect the results since they did not provide valid outcome measures (Muraro 1995; Shah 1993; Vassily 1999). The exclusion of Montorsi 1993 affected the incidence of adverse events. In Montorsi 1993, there were no adverse events in the participants who received the procedure (all participants received the procedure in different regimens). 


\section{Transurethral thermotherapy}

The exclusion of Nickel 1996 resulted in no evidence for the comparison of transurethral thermotherapy versus sham procedure.

\section{DISCUSSION}

\section{Summary of main results}

We included 38 unique studies with 3290 men with CP/CPPS across 23 comparisons. We included all comparisons with short-term follow-up in the analyses. The median age of the participants was 37 years.

We found moderate quality evidence that acupuncture and extracorporeal shockwave therapy probably leads to a clinically meaningful reduction in prostatitis symptoms based on an $\mathrm{NIH}-$ CPSI reduction of greater than three (but less than six). These interventions may not have been associated with an increased incidence of adverse events. We also found that circumcision probably decreased prostatitis symptoms; however, the effect would be small. We found moderate-quality evidence that tibial nerve stimulation probably caused an important decrease in prostatitis symptoms, but we have no information regarding adverse events.

Additionally, we found low- to very low-quality evidence that physical activity, biofeedback, ultrasound and transrectal thermotherapy may have reduced prostatitis symptoms. We were uncertain about the effects of lifestyle interventions, use of an electromagnetic chair, sono-electromagnetic therapy, external radiofrequency hyperthermia, prostatic massage, laser therapy, myofascial trigger point release therapy, osteopathy, TENS, transurethral thermotherapy, transurethral needle ablation, hypercapnic hypoxia and TaiJiQuan. We found no information regarding psychological support or prostatic surgery.

\section{Overall completeness and applicability of evidence}

Our review focused on men with CP/CPPS. Almost all the included studies used the consistent inclusion criteria defined by the $\mathrm{NIH}$ (Nyberg 1999). These diagnostic criteria are related to clinical practice, since these participants are usually tested for urological diseases that could mimic CP/CPPS. However, some of the impact of the NIH consensus on the diagnosis of CP/CPPS might have changed the classification of participants across time (Krieger 2002), therefore, the results of older trials must be interpreted in caution with the current diagnostic criteria. Nevertheless, we incorporated a sensitivity analysis based on this consideration.

We maintained open inclusion criteria for the included interventions. This decision was based on the poorly understood pathophysiological determinants for CP/CPPS (see Background). This led to the inclusion of a wide variety of interventions, which could relate to clinical practice, considering that people with $\mathrm{CP} /$ CPPS usually try different treatment options before achieving some form of relief. However, while this review can be used to discuss the different treatment options with patients, the description of some of these interventions was sometimes insufficient. This was particularly important for more complex interventions such as nonpharmacological interventions (Hoffmann 2013). This could pose a threat to the open discussion of the implications of implementing a certain intervention in clinical practice.
Our review focused on critical patient-important outcomes; however, most studies did not report them consistently, especially the incidence of adverse events. This is a common problem in clinical trials (loannidis 2001), and poses difficulties when estimating the net benefit of the interventions. Additionally, most of the included studies did not report the effects of their interventions on QoL, sexual function and mental health, considering the important impact of CP/CPPS in these domains (Krsmanovic 2014). We acknowledge that the NIH-CPSI score includes QoL and urinary symptoms subscores that, alongside the pain subscore, contribute to a total score that has proven to be valid and reliable (Litwin 1999; McNaughton 2001; Propert 2006; Turner 2003); however, these subscores have not been validated for these constructs individually. We acknowledge that the validated scores for QoL, urinary symptoms, sexual dysfunction, and anxiety and depression might have not been validated in the subpopulation of men with CP/CPPS, therefore their values should be carefully interpreted, specially using the assessment of the MCID.

Furthermore, most studies reported results at four to 12 weeks' follow-up, which might be insufficient considering that men with $\mathrm{CP} / \mathrm{CPPS}$ generally have a long history of symptoms, usually for years (Clemens 2015).

We had planned to explore the effect of the interventions in subgroups of men with different degrees of disease severity and in subgroups of men with common comorbidities (Gasperi 2017), but the included studies provided insufficient data in this regard.

Some of the included treatments for the main comparisons expressed in the 'Summary of findings' table have not been part of routine care for CP/CPPS, especially circumcision (Summary of findings 3 ) and the electromagnetic chair (Summary of findings 4). For this statement, we used, as an example, the recommendations by the European Association of Urology, available at uroweb.org/ guideline/chronic-pelvic-pain/ (last accessed December 2017). As the guideline authors stated, careful considerations should be placed when considering the applicability of these interventions in daily practice. The evidence for the electromagnetic chair is inconsistent for the main outcomes (very low-quality evidence). For circumcision, the effect size was small for the main outcomes (moderate-quality evidence). Furthermore, circumcision is the only intervention included in this review that entails an irreversible surgical procedure. As the Evidence to Decision Framework stated, a careful evaluation of patient's values and preferences, resource use and equity issues and the acceptability and feasibility of the interventions is warranted when drafting recommendations (Alonso-Coello 2016).

\section{Quality of the evidence}

The main limitations of the body of evidence were the following.

- Study limitations: most studies had problems when masking the interventions to study personnel and participants. This is particularly important for this review considering that all the predefined outcomes were subjective participant-reported outcomes. Additionally, most studies poorly reported random sequence generation and allocation concealment and most of the studies had no published protocol or analysis plan to assess the risk of selective reporting. For the comparison of ESWT, we decided not to downgrade due to study limitations since the 
results from studies with high risk of bias were consistent with those with low/unclear risk of bias.

- Inconsistency: we performed few meta-analyses and, in many cases, we found considerable heterogeneity; only in one case we found that the inconsistency was explained to a difference in the criteria for disease definition.

- Imprecision: we estimated an optimal information size of 74 for the primary outcome 'prostatitis symptoms' and 50\% of the studies had a smaller sample size; therefore, most studies had imprecision. Nevertheless, in some cases, meta-analysis of some of the included studies increased the number of participants for each comparison, overcoming this limitation. This was more difficult to do for the outcome 'adverse events' in which the small number of events (in some cases there was a "zero count") resulted in considerable imprecision.

- Publication bias: due to the small number of studies in each comparison, we could not assess the risk of publication bias.

\section{Potential biases in the review process}

We strictly followed our published protocol to reduce the risk of bias in the conduct of this review. Nevertheless, we had to make further specifications due to the multiplicity of comparisons and available outcomes to review. These specifications did not imply major changes and they were done to provide clarity when summarising multiple comparisons (see Differences between protocol and review), especially when drafting the 'Summary of findings' tables. We acknowledge that we might have made 'Summary of findings' tables of each comparison, but this would not have provided further clarity to the understanding of the findings. We also highlight that even though there were no 'Summary of findings' tables for some comparisons, all the findings were rated using GRADE methods.

We performed a comprehensive search in multiple databases, trial registries and other sources to reduce the risk of meta-bias in our review. Additionally, we did not restrict the searches or inclusion of studies on the basis of language of publication; for this purpose, we incorporated three authors with expertise in Chinese, Russian and Korean. Nevertheless, we were unable to retrieve some of the studies on acupuncture published in some Chinese journals that were included in other reviews that searched Chinese Databases (see Agreements and disagreements with other studies or reviews). Should we find the report of these studies, they will be evaluated for inclusion in updates of this review, since we have no clear information regarding their eligibility. Nevertheless, the reported results of these studies in the corresponding reviews were consistent with our findings.

We contacted study authors on multiple occasions with a variable rate of response. However, we acknowledge that many of the ratings of 'unclear' risk of bias were due to limitations in the report of the studies, rather than a true risk of bias in the conduct of these trials.

We deleted the 'Clinical Phenotyping' item as an intervention in our review, as suggested by a peer reviewer. We acknowledge that this strategy might not constitute in itself an intervention. However, this change has not affected the results of this review since we found no trials on this subject. We also modified the presentation of some of the methods of this review (assessment of outcomes and GRADE methods), but this did not affect the results of the review either (see Differences between protocol and review).

\section{Agreements and disagreements with other studies or reviews}

We found several systematic reviews addressing interventions for CP/CPPS; however, only a few of them incorporated nonpharmacological interventions.

A systematic review by Erickson 2008 and its update by Le 2011 included some of the studies for non-pharmacological interventions for $\mathrm{CP} / \mathrm{CPPS}$. These reviews indicated that there was no evidence for the use of biofeedback; however, in our review, we included a clinical trial assessing this intervention. These reviews also included some of the evidence for transurethral thermotherapy and prostatic massage with similar results to ours.

Two systematic reviews included both pharmacological and nonpharmacological interventions (McNaughton 2000; McNaughton 2001). We included two studies from these reviews that were not retrieved by our search strategy (Shah 1993; Vassily 1999); however, we did not include one study on transrectal thermotherapy since the participants in this study did not meet the CP/CPPS criteria (Strohmaier 1988).

The systematic reviews by Cohen 2012; Qin 2016b; and Magistro 2016 included both pharmacological and non-pharmacological interventions. They included some of the studies in this review addressing the effects of sono-electromagnetic therapy, ESWT, acupuncture, aerobic exercise and tibial nerve stimulation, with the same results.

The systematic review by Mishra 2008 focused only on prostatic massage and incorporated one of the trials in our review in addition to several case series.

The systematic review by Chang 2016 addressing acupuncture for CP/CPPS incorporated three additional studies from Chinese journals and failed to include one of the trials in our review since the review searched Chinese databases. One of these additional included studies was excluded in our review since it used a quasirandomised allocation (Ma 2015). We asked the study authors for the remaining two studies. Nevertheless, the two metaanalyses reported by this review yielded similar results in terms of reduction of prostatitis symptoms (NIH-CPSI scores) and an increased response rate with acupuncture in comparison to sham procedures or medical therapy, with a similar incidence of adverse events. Another systematic review by Liu 2016 also focusing on acupuncture for CP/CPPS incorporated four additional studies from Chinese journals (different from those found by Chang 2016) and failed to include one of the trials in our review. One of these additional included studies was also Ma 2015. We asked the study authors for the remaining three studies. Nevertheless, the two meta-analysis reported by this review yielded similar results in terms of reduction of prostatitis symptoms (NIH-CPSI scores) and an increased response rate with acupuncture in comparison to sham procedures or medical therapy, with a similar incidence of adverse events. Furthermore, a systematic review by Qin 2016a included three additional trials (one of them not identified in the previous review); however, the reported meta-analysis yielded similar results in terms of reduction of prostatitis symptoms $(\mathrm{NIH}-$ CPSI scores). We asked the study authors for the remaining studies. 
Finally, a review by Posadzki 2012 on acupuncture for CP/CPPS included six additional studies (different from those found in Chang 2016; Liu 2016; and Qin 2016a). We asked the study authors for the remaining studies. Nevertheless, the meta-analysis reported by this review yielded similar results in terms of an increased response rate with acupuncture in comparison to sham procedures or medical therapy, with a similar incidence of adverse events (this review also highlighted the poor reporting of adverse events in the literature).

\section{AUTHORS' CONCLUSIONS}

\section{Implications for practice}

Based on the findings of moderate quality evidence, this review found that some non-pharmacological interventions such as acupuncture, circumcision, extracorporeal shockwave therapy and tibial nerve stimulation can cause a decrease in prostatitis symptoms and may not be associated with a greater incidence of adverse events. However, given the large qualitative differences of the interventions, they must be assessed in each clinical context, considering the men's values and preferences, their acceptability, the feasibility of their implementation and the use of resources.

There is greater uncertainty regarding the effectiveness of other therapies such as: lifestyle modifications, physical activity, prostatic massage, electromagnetic chair, transrectal thermotherapy, sono-electromagnetic therapy, ultrasound therapy, biofeedback, external radiofrequency, laser therapy, myofascial trigger point release, osteopathy, trans-electrical nerve stimulation, transurethral thermotherapy, transurethral needle ablation, hypercapnic hypoxia and TaiJiQuan. While there is lowquality evidence of the effects of some of these interventions such as lifestyle changes and physical activity, they are relatively safe and they are recommended as part of a healthy lifestyle for multiple conditions. Conversely, prostatic massage has been widely used for CP/CPPS and we still have great uncertainty about its effect and, therefore, it should be carefully assessed before being recommended. The remaining interventions are still experimental.

\section{Implications for research}

Given the low-quality evidence of the research included in this review, there is need for additional research with regards to the effects of some of the included interventions considering that:
- future clinical trials should include a full report of their methods for greater transparency regarding potential sources of bias. Additionally, acknowledging the subjective nature of the participant-reported outcomes that are critical for decision making, masking of these interventions is warranted;

- future studies should include a greater variety of outcomes, including sexual dysfunction, quality of life, and depression and anxiety since this would represent the actual impact of these interventions on participants with CP/CPPS. Additionally, longterm follow-up is needed, recognising the chronicity of this condition. It also is essential for studies to report the incidence of adverse events to assess the net benefit of the interventions;

- ideally, studies should include more than 74 participants to reduce imprecision of the findings when considering prostatitis symptoms using the National Institute of Health - Chronic Prostatitis Symptom Index (NIH-CPSI) score. Larger sample sizes are required when assessing the number of responders to treatment or other outcomes;

- it is important that future studies describe all the details and implications of the interventions, to guarantee reproducibility;

- considering the high comorbidity of participants with CP/CPPS, it is important for studies to include participants with comorbid conditions to assess subgroup effects.

\section{ACKNOWLEDGEMENTS}

We thank the following people and organisations.

- Cochrane Urology Group for providing guidelines and templates for protocol and review development, especially Alea Miller and Phillip Dahm who provided close feedback and advice.

- Instituto Universitario Hospital Italiano de Buenos Aires Cochrane Collaborating Centre, Argentina.

- Family and Community Medicine Service and Urology Service, Hospital Italiano de Buenos Aires, Argentina.

- Iván Solá and Jennifer Lyon for their contribution to the search strategy.

- Jonathan Rees, Giuseppe Magistro and Florian Wagenlehner for their peer-review input of the protocol.

- Federico Tirapegui contributed to the 'Background' section of the protocol and assisted in the data extraction tasks.

- Byung-Cheol Lee, Giuseppe Magistro and Jordan Dimitrakoff for their peer-review input of the review. 


\section{REFERE N CES}

\section{References to studies included in this review}

Ateya 2006 \{published data only\}

Ateya A, Fayez A, Hani R, Zohdy W, Gabbar MA, Shamloul R. Evaluation of prostatic massage in treatment of chronic prostatitis. Urology 2006;67(4):674-8.

\section{Chen 2009 \{published data only\}}

Chen ZX. Observation on therapeutic effect of warm needle moxibustion on chronic non-bacterial prostatitis. Zhongguo Zhen Jiu [Chinese Acupuncture \& Moxibustion] 2009;29(4):275-8.

\section{Fang 2005 \{published data only\}}

Fang J, Xu G, Ding Q, Zhang Y-F. Observation on the safety and efficacy of He-Ne laser equipment on chronic abacteria prostatitis. Fudan University Journal of Medical Sciences 2005;32(2):234-5, 252

\section{Fitzgerald 2013 \{published data only\}}

Badillo SA, Neville CE, Kotarinos R, Fortman C, Fraser L, Cosby A, et al. Randomized multicenter pilot trial shows benefit of manual physical therapies in treatment of urologic chronic pelvic pain. Journal of Women's Health Physical Therapy 2009;33(1):23.

FitzGerald MP, Anderson RU, Potts J, Payne CK, Peters KM, Clemens JQ, et al. Randomized multicenter feasibility trial of myofascial physical therapy for the treatment of urological chronic pelvic pain syndromes. Journal of Urology 2009;182(2):570-80.

Fitzgerald MP, Anderson RU, Potts J, Payne CK, Peters KM, Clemens JQ, et al. Randomized multicenter feasibility trial of myofascial physical therapy for the treatment of urological chronic pelvic pain syndromes. Journal of Urology 2013;189(1 Suppl):S75-85.

\section{Gallo 2014 \{published data only\}}

Gallo L. Effectiveness of diet, sexual habits and lifestyle modifications on treatment of chronic pelvic pain syndrome. Prostate Cancer and Prostatic Diseases 2014;17(3):238-45.

\section{Gao 2012 \{published data only\}}

Gao M, Ding H, Zhong G, Lu J, Wang H, Li Q, et al. The effects of transrectal radiofrequency hyperthermia on patients with chronic prostatitis and the changes of MDA, NO, SOD, and $\mathrm{Zn}$ levels in pretreatment and posttreatment. Urology 2012;79(2):391-6.

\section{Giubilei 2007 \{published data only\}}

Giubilei G, Mondaini N, Minervini A, Saieva C, Lapini A, Serni $S$, et al. Physical activity of men with chronic prostatitis/ chronic pelvic pain syndrome not satisfied with conventional treatments - could it represent a valid option? The physical activity and male pelvic pain trial: a double-blind, randomized study. Journal of Urology 2007;177(1):159-65.

\section{Kabay 2009 \{published data only\}}

Kabay S, Kabay SC, Yucel M, Ozden H. Efficiency of posterior tibial nerve stimulation in category IIIB chronic prostatitis/ chronic pelvic pain: a Sham-Controlled Comparative Study. Urologia Internationalis 2009;83(1):33-8.

Kaikai 2014 \{published data only\}

Kaikai C, Rui W, Yong C, Zhu C, Wei H, Suogang W, et al. Combination therapy of non-intrusive ultrasound with integrated Chinese-western medicine for chronic prostatitis/ chronic pelvic pain syndrome. Chinese Journal of Andrology 2014;28(12):41-4.

\section{Kastner 2004 \{published data only\}}

Kastner C, Hochreiter W, Huidobro C, Cabezas J, Miller P. Cooled transurethral microwave thermotherapy for intractable chronic prostatitis - results of a pilot study after 1 year. Urology 2004;64(6):1149-54.

\section{Kessler 2014 \{published data only\}}

Kessler TM, Mordasini L, Weisstanner C, Juni P, da Costa BR, Wiest R, et al. Sono-electro-magnetic therapy for treating chronic pelvic pain syndrome in men: a randomized, placebocontrolled, double-blind trial. PloS One 2014;9(12):e113368.

Weisstanner C, Mordasini L, Thalmann GN, Verma RK, Rummel C, Federspiel A, et al. Therapy-related longitudinal brain perfusion changes in patients with chronic pelvic pain syndrome. Swiss Medical Weekly 2017;147:w14454.

\section{Kucuk 2015 \{published data only\}}

Kucuk EV, Suceken FY, Bindayi A, Boylu U, Onol FF, Gumus E. Effectiveness of acupuncture on chronic prostatitischronic pelvic pain syndrome category IIIB patients: a prospective, randomized, nonblinded, clinical trial. Urology 2015;85(3):636-40.

\section{Lee 2008 \{published data only\}}

Lee SW, Liong ML, Yuen KH, Krieger JN. Acupuncture and immune function in chronic prostatitis/chronic pelvic pain syndrome: a randomized, controlled study. Complementary Therapies in Medicine 2014;22(6):965-9.

Lee SW, Liong ML, Yuen KH, Leong WS, Chee C, Cheah PY, et al. Acupuncture versus sham acupuncture for chronic prostatitis/chronic pelvic pain. American Journal of Medicine 2008;121(1):79.e1-7.

Lee SW, Liong ML, Yuen KH, Leong WS, Khan NK, Krieger JN. Validation of a sham acupuncture procedure in a randomised, controlled clinical trial of chronic pelvic pain treatment. Acupuncture in Medicine 2011;29(1):40-6.

Lee 2009 \{published data only\}

Lee SH, Lee BC. Electroacupuncture relieves pain in men with chronic prostatitis/chronic pelvic pain syndrome: three-arm randomized trial. Urology 2009;73(5):1036-41.

\section{Leskinen 2002 \{published data only\}}

Leskinen MJ, Kilponen A, Lukkarinen O, Tammela TL. Transurethral needle ablation for the treatment of chronic pelvic pain syndrome (category III prostatitis): a randomized, sham-controlled study. Urology 2002;60(2):300-4. 


\section{Marx 2009 \{published data only\}}

Marx S. ICAOR 2006: does osteopathic treatment have an influence on the symptoms of patients with chronic prostatitis/ chronic pelvic pain syndrome (CPPS)? A randomized controlled trial. International Journal of Osteopathic Medicine 2006;9(1):44.

Marx S, Cimniak U, Beckert R, Schwerla F, Resch KL. Chronic prostatitis/chronic pelvic pain syndrome. Influence of osteopathic treatment - a randomized controlled study [Chronische Prostatitis/chronisches Beckenschmerzsyndrom. Einfluss osteopathischer Behandlungen - eine randomisiert kontrollierte Studie]. Der Urologe. Ausg. A 2009;48(11):1339-45.

\section{Montorsi 1993 \{published data only\}}

Montorsi F, Guazzoni G, Bergamaschi F, Galli L, Consonni P, Matozzo V, et al. Is there a role for transrectal microwave hyperthermia of the prostate in the treatment of abacterial prostatitis and prostatodynia?. Prostate 1993;22(2):139-46.

\section{Muraro 1995 \{published data only\}}

Muraro GB. Clinical study on the efficacy and safety of seaprose $S$ combined with local prostatic hyperthermia in chronic nonbacterial prostatitis. Controlled study versus local prostatic hyperthermia [Studio clinico sull'efficacia e la tollerabilita del seaprose $\mathrm{S}$ associato a ipertermia locale prostatica nella prostatite cronica abatterica. Studio controllato verso ipertermia locale prostatica]. Archivio di Medicina Interna 1995;47(2-3):73-86.

\section{Neimark 2016 \{published data only\}}

Neimark Al, Maksimova SS. Effect of hypercapnic hypoxia on chronic abacterial prostatitis. Urologiia (Moscow, Russia : 1999) 2016;1(3):80-4. [PUBMED: 28247635]

\section{Nickel 1996 \{published data only\}}

Nickel JC, Sorensen R. Transurethral microwave thermotherapy for nonbacterial prostatitis: a randomized double-blind sham controlled study using new prostatitis specific assessment questionnaires. Journal of Urology 1996;155(6):1950-4; discussion 1954

\section{Oh 2009 \{published data only\}}

Oh MM, Jin MH, Moon DG, Nam SG, Bae JH, Park HS. A prospective, randomized, placebo controlled study of radiofrequency therapy for the treatment of chronic prostatitis/chronic pelvic pain syndrome. Journal of Urology 2009;181(4):122.

\section{Paick 2006 \{published data only\}}

Paick JS, Lee SC, Ku JH. More effects of extracorporeal magnetic innervation and terazosin therapy than terazosin therapy alone for non-inflammatory chronic pelvic pain syndrome: a pilot study. Prostate Cancer and Prostatic Diseases 2006;9(3):261-5.

\section{Pajovic 2016 \{published data only\}}

Pajovic B, Radojevic N, Dimitrovski A, Vukovic M. Comparison of the efficiency of combined extracorporeal shock-wave therapy and triple therapy versus triple therapy itself in Category III B chronic pelvic pain syndrome (CPPS). Aging Male 2016;19(3):1-6.

\section{Rowe 2005 \{published data only\}}

Rowe E, Smith C, Laverick L, Elkabir J, Witherow RO, Patel A. A prospective, randomized, placebo controlled, double-blind study of pelvic electromagnetic therapy for the treatment of chronic pelvic pain syndrome with 1 year of followup. Journal of Urology 2005;173(6):2044-7.

\section{Sahin 2015 \{published data only\}}

Sahin S, Bicer M, Eren GA, Tas S, Tugcu V, Tasci Al, et al. Acupuncture relieves symptoms in chronic prostatitis/chronic pelvic pain syndrome: a randomized, sham-controlled trial. Prostate Cancer and Prostatic Diseases 2015;18(3):249-54.

\section{Samhan 2011 \{published data only\}}

Samhan Ahmed F, Abd-Elhalim Nermeen M, Elnegmy Emam H, Roiah Mohamed M. The effect of transcutaneous electrical nerve stimulation in the treatment of chronic pelvic pain syndrome: an evidence based electromyographic studies. Indian Journal of Physiotherapy \& Occupational Therapy 2011;5(3):14-7.

\section{Shah 1993 \{published data only\}}

Shah T, Watson G, Barnes D. Microwave hyperthermia in the treatment of chronic abacterial prostatitis and prostatodynia: results of a double blind placebo controlled trial (Abstract). Journal of Urology 1993;149:405A.

\section{Shen 2006 \{published data only\}}

Shen SL, He DL, Luo Y. Clinical trials of combined therapy of an oral Chinese medicine with massage for chronic nonbacterial prostatitis. Zhonghua Nan Ke Xue 2006;12(9):851-3.

\section{Sikiru 2008 \{published data only\}}

Sikiru L, Shmaila H, Muhammed SA. Transcutaneous electrical nerve stimulation (TENS) in the symptomatic management of chronic prostatitis/chronic pelvic pain syndrome: a placebocontrol randomized trial. International Brazilian Journal of Urology 2008;34(6):708-13; discussion 714 .

\section{Vahdatpour 2013 \{published data only\}}

Moayednia A, Haghdani S, Khosrawi S, Yousefi E, Vahdatpour B. Long-term effect of extracorporeal shock wave therapy on the treatment of chronic pelvic pain syndrome due to non bacterial prostatitis. Journal of Research in Medical Sciences 2014;19(4):293-6.

* Vahdatpour B, Alizadeh F, Moayednia A, Emadi M, Khorami MH, Haghdani S. Efficacy of extracorporeal shock wave therapy for the treatment of chronic pelvic pain syndrome: a randomized, controlled trial. ISRN Urology 2013;2013:972601.

\section{Vassily 1999 \{published data only\}}

Vassily O, Andrey S, Evgenii D, Otabek I, Boris L, Sergey R. Efficacy of transrectal microwave hyperthemia (TRMH) in the treatment of chronic prostatitis. A randomized sham controlled comparative study (Abstract). Journal of Urology 1999;161(4 Suppl):33A.

\section{Wang 2002 \{published data only\}}

Wang J, Li J, Lu R, Wang JM. Treatment of external RF hyperthermia combining with alpha 1-adrenergic receptor 
blocker for patients with prostatodynia and chronic nonbacterial prostatitis. Zhonghua Nan Ke Xue 2002;8(1):48-50.

Yang 2011 \{published data only\}

Yang ZS, Zu XB, Qi L, Song LM, Liu TR. Combination therapy of biofeedback with electrical stimulation for chronic prostatitis/chronic pelvic pain syndrome. Zhonghua Nan Ke Xue 2011;17(7):611-4.

\section{Yoo 2009 \{published data only\}}

* Chung H, Choi H, Yoo TK, Cho JM, Kim HS. The effects of microwave thermotherapy for chronic prostatitis/chronic pelvic pain syndrome: a prospective, randomized study. Urogenital Tract Infection 2017;12(1):35-41.

Kim H, Chung H, Yang S, Yoo T, Cho J. Effects of thermotherapy and medical therapy for chronic prostatitis/chronic pelvic pain syndrome: a prospective study. Urology 2011;78(3):S85-6.

Yoo TK, Moon KT, Lee SW, Kim HS, Chung H, Kim JY. Effect of the combination treatment of URO-DrG and medical therapy for chronic prostatitis/chronic pelvic pain syndrome: a prospective study. Journal of Urology 2009;181(4):122.

\section{Zeng 2012 \{published data only\}}

Zeng X, Ye Z, Yang W, Chen Z, Du G. Extracorporeal shock wave treatment for noninflammatory chronic pelvic pain syndrome: a prospective, randomized, sham-controlled study. Journal of Urology 2009;181(4):122-3.

Zeng XY, Liang C, Ye ZQ. Extracorporeal shock wave treatment for non-inflammatory chronic pelvic pain syndrome: a prospective, randomized and sham-controlled study. Chinese Medical Journal 2012;125(1):114-8.

\section{Zhang 2011a \{published data only\}}

Zhang DY, Pan XC. Chinese medicine union Taijiquan treatment chronicity non-bacterial prostatitis curative effect observation. Chinese Manipulation \& Rehabilitation Medicine 2011;15(2):181.

\section{Zhao 2015 \{published data only\}}

Zhao Y, Zhao W, Lang G, Chen Y, Liu J, Wang G, et al. Circumcision plus antibiotic, anti-inflammatory, and alphablocker therapy for the treatment for chronic prostatitis/chronic pelvic pain syndrome: a prospective, randomized, multicenter trial. World Journal of Urology 2015;33(5):617-22.

\section{Zimmermann 2009 \{published data only\}}

* Zimmermann R, Cumpanas A, Miclea F, Janetschek G. Extracorporeal shock wave therapy for the treatment of chronic pelvic pain syndrome in males: a randomised, double-blind, placebo-controlled study. European Urology 2009;56(3):418-24.

Zimmermann RP, Cumpanas A, Miclea F, Janetschek G. One year follow up of extracorporeal shock wave therapy (ESWT) for chronic pelvic pain syndrome (CPPS) in a randomised placebocontrolled double-blind study. European Urology (Suppl) 2010;9(2):214.

\section{References to studies excluded from this review}

Aboumarzouk 2012 \{published data only\}

Aboumarzouk OM, Nelson RL. Pregabalin for chronic prostatitis. Cochrane Database of Systematic Reviews 2012, Issue 8. [DOI: 10.1002/14651858.CD009063.pub2]

Aliaev 2006 \{published data only\}

Aliaev luG, Vinarov AZ, Lokshin KL, Spivak LG. Efficiency and safety of prostamol-Uno in patients with chronic abacterial prostatitis. Urologiia 2006;1(1):47-50.

\section{Allen 2017 \{published data only\}}

Allen S, Aghajanyan IG. Effect of thermobalancing therapy on chronic prostatitis and chronic pelvic pain syndrome. Journal of Clinical Urology 2017;10(4):347-54.

Allen S, Aghajanyan IG. New independent thermobalancing treatment with therapeutic device for chronic prostatitis/ chronic pelvic pain syndrome. Nephro-Urology Monthly 2017;9(2):1-6.

\section{Anothaisintawee 2011 \{published data only\}}

Anothaisintawee T, Attia J, Nickel JC, Thammakraisorn S, Numthavaj P, McEvoy M, et al. Management of chronic prostatitis/chronic pelvic pain syndrome: a systematic review and network meta-analysis. JAMA 2011;305(1):78-86.

\section{Barbalias 1998 \{published data only\}}

Barbalias GA, Nikiforidis G, Liatsikos EN. Alpha-blockers for the treatment of chronic prostatitis in combination with antibiotics. Urologia Internationalis 1998;159(3):883-7.

\section{Bschleipfer 2007 \{published data only\}}

Bschleipfer T, Wagenlehner FM, Weidner W. Intraprostatic botulinum toxin $\mathrm{A}$ injection in chronic prostatitis and chronic pelvic pain syndrome (CP/CPPS) [Intraprostatische Botulinumtoxin-A-Injektion bei chronischer Prostatitis und chronischem Beckenschmerzsyndrom (CP/CPPS)]. Der Urologe Ausg. A 2007;46(9):1030-2.

Department of Urology, Pediatric Urology - Justus-LiebigUniversity Giessen. Intraprostatic injection of Botulinumtoxin type $A$ in patients with chronic prostatitis / chronic pelvic pain syndrome (CP/CPPS) - CP(BTX)PS. https:// www.clinicaltrialsregister.eu/ctr-search/trial/2007-001602-24/ DE (accessed 22 January 2018) 2009.

\section{Capodice 2005 \{published data only\}}

Capodice JL, Bemis DL, Buttyan R, Kaplan SA, Katz AE. Complementary and alternative medicine for chronic prostatitis/chronic pelvic pain syndrome. Evidencebased Complementary and Alternative Medicine : ECAM 2005;2(4):495-501.

\section{Chambo 2009 \{published data only\}}

Chambo RC, Carvalho PE, Cataneo AJM. Effectiveness and safety of finasteride in male chronic pelvic pain syndrome. Cochrane Database of Systematic Reviews 2009, issue 2. [DOI: 10.1002/14651858.CD007723.pub2] 
Chang 2016 \{published data only\}

Chang SC, Hsu CH, Hsu CK, Yang SS, Chang SJ. The efficacy of acupuncture in managing patients with chronic prostatitis/ chronic pelvic pain syndrome: a systemic review and metaanalysis. Neurourology and Urodynamics 2016;1:474-81.

\section{Chen 2006 \{published data only\}}

Chen J, Hu L. Traditional Chinese medicine for the treatment of chronic prostatitis in China: a systematic review and metaanalysis. Journal of Alternative and Complementary Medicine (New York, N.Y.) 2006;12(8):763-9.

\section{Chuang 2006 \{published data only\}}

Chuang YC, Chancellor MB. The application of botulinum toxin in the prostate. Journal of Urology 2006;176(6 Pt 1):2375-82.

\section{Cohen 2012 \{published data only\}}

Cohen JM, Fagin AP, Hariton E, Niska JR, Pierce MW, Kuriyama A, et al. Therapeutic intervention for chronic prostatitis/chronic pelvic pain syndrome (CP/CPPS): a systematic review and metaanalysis. PloS One 2012;7(8):e41941.

\section{Colleen 1975 \{published data only\}}

Colleen S, Mrdh PA. Effect of metacycline treatment on non-acute prostatitis. Scandinavian Journal of Urology and Nephrology 1975;9(3):198-204.

\section{DRKS00009352 \{published data only\}}

DRKS00009352. Effect of the physiotherapeutic device and thermobalancing therapy compared with no therapy on pain, urinary symptoms, quality of life and prostate volume in men with chronic prostatitis / chronic pelvic pain syndrome (CP/CPPS). http://www.drks.de/drks_web/navigate.do? navigationld=trial.HTML\&TRIAL_ID=DRKS00009352 (accessed 22 January 2018 via http://apps.who.int/trialsearch/ search portal).

\section{Erickson 2008 \{published data only\}}

Erickson BA, Schaeffer AJ, Van Le B. Chronic prostatitis. BMJ Clinical Evidence 2008;2008:1802.

\section{Evliyaoglu 2002 \{published data only\}}

Evliyaoglu Y, Burgut R. Lower urinary tract symptoms, pain and quality of life assessment in chronic non-bacterial prostatitis patients treated with alpha-blocking agent doxazosin; versus placebo. International Urology and Nephrology 2002;34(3):351-6.

\section{Feng 2011 \{published data only\}}

Feng H, Hu C, Li L. Influence of whole-range systematic nursing intervention of therapeutic effect and compliance of patients with chronic prostatitis. Chinese Nursing Research 2011;25(5A):1146-8.

\section{Galeone 2012 \{published data only\}}

Galeone G, Spadavecchia R, Balducci MT, Pagliarulo V. The role of Proxelan in the treatment of chronic prostatitis. Results of a randomized trial [Ruolo di Proxelan nel trattamento delle prostatitit croniche. Risultati di un trial randomizzato]. Minerva Urologica e Nefrologica [Italian Journal of Urology and Nephrology] 2012;64(2):135-41.

\section{Glybochko 2014 \{published data only\}}

Glybochko PV, Alyaev Yu G, Chalyj ME, Voskanyan GA. Primary assessment of electrode pharmaphoresis efficacy in treatment of chronic bacterial prostatitis. European Urology Supplements 2014;13(1):e571.

Golubchikov 2005 \{published data only\}

Golubchikov VA, Sitnikov NV, Kochetov AG, Perekhodov SN, Sidorov OV, Roiuk RV, et al. Optimal treatment of benign prostatic hyperplasia (BPH) with comorbid chronic prostatitis (category IIIA). Urologiia 2005;1(4):9-12.

\section{Hong 2008 \{published data only\}}

Hong JY, Zhang YY. Observation on therapeutic effect of abdominal cluster-needling on chronic non-bacterial prostatitis. Chinese Acupuncture \& Moxibustion 2008;28(1):24-6.

\section{Ikeuchi 1990 \{published data only\}}

Ikeuchi T. Clinical studies on chronic prostatitis and prostatitislike syndrome (4). The kampo treatment for intractable prostatitis. Hinyokika Kiyo 1990;36(7):801-6.

ISRCTN43221600 \{published data only\}

ISRCTN43221600. Pilot study for the evaluation of a combined psycho- and physiotherapeutic treatment program for patients with chronic pelvic pain syndrome (CPPS) www.isrctn.com/ISRCTN43221600 (accessed 4 November 2017). [ISRCTN43221600]

\section{Jimenez-Pacheco 2014 \{published data only\}}

Jimenez-Pacheco A, Jimenez-Pacheco A, Nogueras-Ocaña M. Analysis of therapeutic alternatives in the treatment of chronic prostatitis/syndrome chronic pelvic pain. Urology 2014;84(4):S241.

\section{Kalinina 2015 \{published data only\}}

Kalinina SN, Koren'kov DG, Fesenko VN, Demidov DA, Tiktinskij NO. Pathogentic treatment of chronic nonbacterial prostatitis complicated by sperm disorders. Urologiia 2015;1(4):64-6, 68.

\section{Kamalov 2006 \{published data only\}}

Kamalov AA, Efremov EA, Dorofeev SD, Paniushkin SM. Use of oral vitaprost in the treatment of chronic abacterial prostatitis. Urologiia 2006;1(5):45-50.

\section{Kogan 2010 \{published data only\}}

Kogan MI, Shangichev AV, Belousov II. Efficacy of magnetolaser therapy of patients with an inflammatory form of chronic abacterial prostatitis. Urologiia 2010;1(2):42-4.

Kotarinos 2009 \{published data only\}

Kotarinos R, Fortman C, Neville C, Badillo S, O'Dougherty B, Fraser $\mathrm{L}$, et al. Physical findings in patients with urologic chronic pelvic pain syndromes (UCPPS). Neurourology and Urodynamics 2009;28(7):911-2

Le 2011 \{published data only\}

Le B, Schaeffer AJ. Chronic prostatitis. BMJ Clinical Evidence 2011;2011:1802. 
Lee 2006 \{published data only\}

Lee HN, Kim JS, Shim BS. The recurrence of chronic pelvic pain syndrome and the role of Uro-Vaxom. Korean Journal of Urology 2006;47(1):42-6.

\section{Lee 2007 \{published data only\}}

Lee SW, Liong ML, Yuen KH, Liong YV, Krieger JN. Chronic prostatitis/chronic pelvic pain syndrome: role of alpha blocker therapy. Journal of Urology 2007;78(2):97-105.

\section{Leng 2007 \{published data only\}}

Leng J, Lv J, Dai S, Chen B, Wang Y. The cocktail treatment of chronic nonbacterial prostatitis. Chinese Journal of Andrology 2007;21(1):26-8.

\section{Liu 2016 \{published data only\}}

Liu BP, Wang YT, Chen SD. Effect of acupuncture on clinical symptoms and laboratory indicators for chronic prostatitis/ chronic pelvic pain syndrome: a systematic review and meta-analysis. International Urology and Nephrology 2016;48(12):1977-91.

\section{Lokshin 2010 \{published data only\}}

Lokshin KL, Alyaev YG, Vinarov AZ, Spivak LG. Randomized open label comparative study of efficacy and safety of combination (ciprofloxacin+doxazosin) versus monotherapy (ciprofloxacin) in patients with category II or category IIIA prostatitis. Urologiia 2010;9(2):140.

\section{Lopatkin 2009 \{published data only\}}

Lopatkin NA, Kamalov AA, Mazo EB, Dorofeev SD, Efremov EA, Kozdoba AS, et al. Administration of oral vitaprost for prevention of exacerbations of chronic abacterial prostatitis. Urologiia 2009;1(1):29-35.

Loran 2003 \{published data only\}

Loran OB, Pushkar' Dlu, Tedeev VV, Nosovitskii PB. Gentos in the treatment of chronic abacterial prostatitis. Urologiia 2003;1(6):30-2.

\section{Ma 2015 \{published data only\}}

Ma Y, Li X, Li F, Yu W, Wang Z. Clinical research of chronic pelvic cavity pain syndrome treated with acupoint catgut embedding therapy. Zhongguo Zhen Jiu 2015;35(6):561-6.

Ma Y, Wang ZL, Sun ZX, Men B, Shen BQ. Efficacy observation on chronic pelvic pain syndrome of damp-heat stagnation pattern treated with acupoint catgut embedding therapy. Zhongguo Zhen Jiu 2014;34(4):351-4.

\section{Magistro 2016 \{published data only\}}

Magistro G, Wagenlehner FM, Grabe M, Weidner W, Stief CG, Nickel JC. Contemporary management of chronic prostatitis/chronic pelvic pain syndrome. European Urology 2016;69(2):286-97.

\section{Marx 2013 \{published data only\}}

Marx S, Cimniak U, Rutz M, Resch KL. Long-term effects of osteopathic treatment of chronic prostatitis with chronic pelvic pain syndrome: a 5-year follow-up of a randomized controlled trial and considerations on the pathophysiological context [Langzeiteffekte osteopathischer Behandlungen bei chronischer Prostatitis/chronischem Beckenschmerzsyndrom: 5-Jahres-Follow-up einer randomisiert kontrollierten Studie und Überlegungen zum pathophysiologischen Kontext]. Der Urologe. Ausg. A 2013;52(3):384-90.

\section{McNaughton 2000 \{published data only\}}

McNaughton Collins M, MacDonald R, Wilt TJ. Diagnosis and treatment of chronic abacterial prostatitis: a systematic review. Annals of Internal Medicine 2000;133(5):367-81.

\section{McNaughton 2001 \{published data only\}}

McNaughton Collins M, MacDonald R, Wilt TJ. Interventions for chronic abacterial prostatitis. Cochrane Database of Systematic Reviews 2001, Issue 1. [DOI: 10.1002/14651858.CD002080]

McNaughton 2002 \{published data only\}

McNaughton Collins M, Wilt TJ. Allopurinol for chronic prostatitis. Cochrane Database of Systematic Reviews 2002, Issue 4. [DOI: 10.1002/14651858.CD001041; CD001041]

\section{Mishra 2008 \{published data only\}}

Mishra VC, Browne J, Emberton M. Role of repeated prostatic massage in chronic prostatitis: a systematic review of the literature. Urology 2008;72(4):731-5.

\section{NCT00194597 \{published data only\}}

NCT00194597. Trial of Viagra' in men with chronic pelvic pain syndrome type III. clinicaltrials.gov/ct2/show/NCT00194597 Date first registered: 19 September 2005.

\section{NCT00194623 \{published data only\}}

NCT00194623. Botox as a treatment for chronic male pelvic pain syndrome. ClinicalTrials.gov/show/NCT00194623 Date first registered: 19 September 2005.

\section{NCT00194636 \{published data only\}}

NCT00194636. Effectiveness of sympathetic plexus block on male pelvic pain (prostatitis, prostatodynia). clinicaltrials.gov/ ct2/show/NCT00194636 Date first registered: 19 September 2005.

\section{NCT00301405 \{published data only\}}

NCT00301405. Open-label study of thalidomide for chronic prostatitis/chronic pelvic pain. clinicaltrials.gov/ct2/show/ NCT00301405 Date first registered: 10 March 2006.

NCT00464373 \{published data only\}

NCT00464373. Botulinum toxin type A for the treatment of male chronic pelvic pain syndrome. clinicaltrials.gov/ct2/show/ NCT00464373 Date first registered: 23 April 2007.

\section{NCT00529386 \{published data only\}}

NCT00529386. Botox for chronic prostatitis/chronic pelvic pain syndrome (CP/CPPS). clinicaltrials.gov/ct2/show/NCT00529386 Date first registered: 14 September 2007.

\section{NCT01678911 \{published data only\}}

NCT01678911. Efficacy of Gralise ${ }^{\circledR}$ for chronic pelvic pain. clinicaltrials.gov/ct2/show/NCT01678911 Date first registered: 5 September 2012 


\section{NCT01830829 \{published data only\}}

NCT01830829. JALYN for benign prostatic hyperplasia (BPH) and chronic prostatitis/chronic pelvic pain syndrome (CP/CPPS). ClinicalTrials.gov/show/NCT01830829 Date first registered: 12 April 2013.

\section{NCT02042651 \{published data only\}}

NCT02042651. A sham controlled study of the effects of ultrasonic shockwaves as a treatment for chronic pelvic pain. ClinicalTrials.gov/show/NCT02042651 Date first registered: 23 January 2014.

\section{Nickel 2011 \{published data only\}}

Nickel JC, Roehrborn C, Montorsi F, Wilson TH, Rittmaster RS. Dutasteride reduces prostatitis symptoms compared with placebo in men enrolled in the REDUCE study. Journal of Urology 2011;186(4):1313-8.

\section{Osborn 1981 \{published data only\}}

Osborn DE, George NJR, Rao PN. Prostatodynia - physiological characteristics and rational management with muscle relaxants. British Journal of Urology 1981;53(6):621-3.

\section{Pavone 2010 \{published data only\}}

Pavone C, Abbadessa D, Tarantino ML, Oxenius I, Lagana A, Lupo A, et al. Associating Serenoa repens, Urtica dioica and Pinus pinaster. Safety and efficacy in the treatment of lower urinary tract symptoms. Prospective study on 320 patients [Associazione di Serenoa repens, Urtica dioica e Pinus pinaster. Sicurezza ed efficacia]. Urologia 2010;77(1):43-51.

\section{Posadzki 2012 \{published data only\}}

Posadzki P, Zhang J, Lee M S, Ernst E. Acupuncture for chronic nonbacterial prostatitis/chronic pelvic pain syndrome: a systematic review. Journal of Andrology 2012;33(1):15-21.

\section{Pushkar' 2006 \{published data only\}}

Pushkar' Dlu, Zaitsev AV, Segal AS. Longidase in the treatment of chronic prostatitis. Urologiia 2006;1(6):26-8.

\section{Qin 2016a \{published data only\}}

Qin Z, Wu J, Zhou J, Liu Z. Systematic review of acupuncture for chronic prostatitis/chronic pelvic pain syndrome. Medicine (Baltimore) 2016;95(11):e3095.

\section{Qin 2016b \{published data only\}}

Qin Z, Wu J, Tian J, Zhou J, Liu Y, Liu Z. Network meta-analysis of the efficacy of acupuncture, alpha-blockers and antibiotics on chronic prostatitis/chronic pelvic pain syndrome. Scientific Reports 2016;6:35737.

\section{Razumov 2005 \{published data only\}}

Razumov SV, Egorov AA. Validity of using physical therapy in combined treatment of chronic prostatitis. Urologiia 2005;1(2):42-6.

\section{Simmons 1985 \{published data only\}}

Simmons PD, Thin RN. Minocycline in chronic abacterial prostatitis: a double-blind prospective trial. British Journal of Urology 1985;57(1):43-5.

\section{Stamatiou 2014 \{published data only\}}

Stamatiou KN, Moschouris H. A prospective interventional study in chronic prostatitis with emphasis to clinical features. Urology Journal 2014;11(4):1829-33.

\section{Takahashi 2005 \{published data only\}}

Takahashi K, Ozaki Y, Yoshida T, Fujimoto N, Matsumoto T, Nakashima M, et al. Clinical evaluation of treatment using mainly levofloxacin and cernitin pollen extract for chronic nonbacterial prostatitis as judged by the National Institutes of Health Chronic Prostatitis Symptom Index. Nishinihon Journal of Urology 2005;67(11):637-48

\section{Thakkinstian 2012 \{published data only\}}

Thakkinstian A, Attia J, Anothaisintawee T, Nickel JC. Alphablockers, antibiotics and anti-inflammatories have a role in the management of chronic prostatitis/chronic pelvic pain syndrome. BJU International 2012;110(7):1014-22.

\section{Thin 1983 \{published data only\}}

Thin RN, Simmons PD. Review of results of four regimens for treatment of chronic non-bacterial prostatitis. British Journal of Urology 1983;55(5):519-21.

\section{Tkachuk 2006 \{published data only\}}

Tkachuk VN, Al'-Shukri SKh, Lotsan-Medvedev AK. Vitaprost efficacy in patients with chronic abacterial prostatitis. Urologiia 2006;1(2):71-2, 74

\section{Tkachuk 2011 \{published data only\}}

Tkachuk VN, Al'-Shukri SKh, Tkacuk IN, Kornienko VI. Correction of erectile dysfunction in patients with chronic abacterial prostatitis. Urologiia 2011;1(6):29-31.

\section{Wagenlehner 2017 \{published data only\}}

Wagenlehner FME, van Till JWO, Houbiers JGA, Martina RV, Cerneus DP, Melis JHJM, et al. Fatty acid amide hydrolase inhibitor treatment in men with chronic prostatitis/chronic pelvic pain syndrome: an adaptive double-blind, randomized controlled trial. Urology 2017;103:191-7. [PUBMED: 28254462]

\section{Xu 2004 \{published data only\}}

Xu S, Qi G, Tang P, Li Y. Combined therapy for the chronic pelvic pain syndrome. Zhonghua Nan Ke Xue 2004;10(6):429-30, 433.

\section{Yang 2006 \{published data only\}}

Yang G, Wei Q, Li H, Yang Y, Zhang S, Dong Q. The effect of alphaadrenergic antagonists in chronic prostatitis/chronic pelvic pain syndrome: a meta-analysis of randomized controlled trials. Journal of Andrology 2006;27(6):847-52.

Yang 2008 \{published data only\}

Yang M, Zhao X, Hou Y. Management of chronic prostatitis/ chronic pelvic pain syndrome (CP/CPPS): a systematic review and meta-analysis of randomized controlled trials (RCTs). Chinese Journal of Andrology 2008;22(4):20-6.

Zhang 2011b \{published data only\}

Zhang MJ, Weng JF, Shi YL, Cheng WJ, Ruan XJ, Zhang QY. Effect of aike mixture on the inflammatory infiltration in patients 
with chronic prostatitis type III A. Chinese Journal of Integrative Medicine 2011;17(1):26-30.

\section{Zhou 2017 \{published data only\}}

Zhou M, Yang M, Chen L, Yu C, Zhang W, Ji J, et al. The effectiveness of long-needle acupuncture at acupoints BL30 and BL35 for CP/CPPS: a randomized controlled pilot study. BMC Complementary and Alternative Medicine 2017;17:1-6.

\section{References to studies awaiting assessment}

\section{Rochester 2011 \{published and unpublished data\}}

* Rochester M, Armitage J, Sanders M, Christmas P. Self management activation randomised trial for prostatitis (SMARTP): study protocol for a randomised controlled trial. Trials 2011;12:210. [ISRCTN21012555]

\section{References to ongoing studies}

ChicTR-IPR-16009262 \{published data only\}

ChiCTR-IPR-16009262. A randomized controlled trial of psychological intervention therapy in patients with category III chronic prostatitis/chronic pelvic pain syndrome. http:// www.chictr.org.cn/showprojen.aspx?proj=15638 (accessed 22 January 2018 via http://apps.who.int/trialsearch/ search portal).

\section{NCT01828996 \{unpublished data only\}}

Shocking Therapy for Chronic Pelvic Pain Syndrome (CPPS).. Ongoing study February 2013..

\section{NCT02588274 \{published and unpublished data\}}

Qin Z, Zang Z, Wu J, Zhou J, Liu Z. Efficacy of acupuncture for chronic prostatitis/chronic pelvic pain syndromes: study protocol for a randomized, sham acupuncture-controlled trial. BMC Complementary and Alternative Medicine 2016;16(1):440. [PUBMED: 27821109]

\section{NCT03213938 \{published data only\}}

NCT03213938. Acupuncture for chronic prostatitis/chronic pelvic pain syndrome: a multicenter randomized controlled trial. clinicaltrials.gov/ct2/show/NCT03213938 Date first registered: 11 July 2017.

\section{Additional references}

\section{Alonso-Coello 2016}

Alonso-Coello P, Schünemann HJ, Moberg J, BrignardelloPetersen R, Akl EA, Davoli M, et al. GRADE Evidence to Decision (EtD) frameworks: a systematic and transparent approach to making well informed healthcare choices. 1: Introduction. BMJ (Clinical Research Ed.) 2016;353:i2016. [PUBMED: 27353417]

\section{Anderson 2008}

Anderson RU, Orenberg EK, Chan CA, Morey A, Flores V. Psychometric profiles and hypothalamic-pituitary-adrenal axis function in men with chronic prostatitis/chronic pelvic pain syndrome. Journal of Urology 2008;179(3):956-60. [PUBMED: 18207189]

\section{Antolak 2002}

Antolak SJ Jr, Hough DM, Pawlina W, Spinner RJ. Anatomical basis of chronic pelvic pain syndrome: the ischial spine and pudendal nerve entrapment. Medical Hypotheses 2002;59(3):349-53. [PUBMED: 12208168]

\section{Arisan 2006}

Arisan ED, Arisan S, Kiremit MC, Tigli H, Caskurlu T, PalavanUnsal N, et al. Manganese superoxide dismutase polymorphism in chronic pelvic pain syndrome patients. Prostate Cancer and Prostatic Diseases 2006;9(4):426-31. [PUBMED: 16847469]

\section{Bajpayee 2012}

Bajpayee P, Kumar K, Sharma S, Maurya N, Kumar P, Singh R, et al. Prostatitis: prevalence, health impact and quality improvement strategies. Acta Poloniae Pharmaceutica 2012;69(4):571-9. [PUBMED: 22876597]

\section{Barry 1995}

Barry MJ, Williford WO, Chang Y, Machi M, Jones K, WalkerCorkery $\mathrm{E}$, et al. Benign prostatic hyperplasia specific health status measures in clinical research: how much change in the American Urological Association symptom index and the benign prostatic hyperplasia impact index is perceptible to patients?. Journal of Urology 1995;154(5):1770-4.

\section{Bartoletti 2007}

Bartoletti R, Cai T, Mondaini N, Dinelli N, Pinzi N, Pavone C, et al. Prevalence, incidence estimation, risk factors and characterization of chronic prostatitis/chronic pelvic pain syndrome in urological hospital outpatients in Italy: results of a multicenter case-control observational study. Journal of Urology 2007;178(6):2411-5; discussion 2415. [PUBMED: 17937946]

\section{Brunton 2011}

Brunton LL, Chabner BA, Knollmann BC. Goodman and Gilman's the Pharmacological Basis of Therapeutics. 12th Edition. New York (NY): McGraw-Hill Education, 2011.

\section{Button 2015}

Button KS, Kounali D, Thomas L, Wiles NJ, Peters TJ, Welton NJ, et al. Minimal clinically important difference on the Beck Depression Inventory-II according to the patient's perspective. Psychological Medicine 2015;45(14):3269-79.

\section{Cates 2015}

Cates C, Karner C. Clinical importance cannot be ruled out using mean difference alone. BMJ (Clinical Research Ed.) 2015;351:h5496. [PUBMED: 26588935]

\section{Chen 2016}

Chen X, Hu C, Peng Y, Lu J, Yang NQ, Chen L, et al. Association of diet and lifestyle with chronic prostatitis/chronic pelvic pain syndrome and pain severity: a case-control study. Prostate Cancer and Prostatic Diseases 2016;19(1):92-9. [PUBMED: 26666410]

\section{Clemens 2015}

Clemens JQ, Clauw DJ, Kreder K, Krieger JN, Kusek JW, Lai HH, et al. Comparison of baseline urological symptoms in men 
and women in the MAPP research cohort. Journal of Urology 2015;193(5):1554-8. [PUBMED: 25463989]

\section{Collins 1998}

Collins MM, Stafford RS, O'Leary MP, Barry MJ. How common is prostatitis? A national survey of physician visits. Journal of Urology 1998;159(4):1224-8. [PUBMED: 9507840]

\section{Corsaletti 2014}

Corsaletti BF, Proença MDGL, Bisca GKW, Leite JC, Bellinetti LM, Pitta F. Minimal important difference for anxiety and depression surveys after intervention to increase daily physical activity in smokers. Fisioterapia e Pesquisa 2014;21:359-64.

\section{Covidence [Computer program]}

Veritas Health Innovation. Covidence systematic review software. Melbourne, Australia: Veritas Health Innovation, 2013.

\section{De La Rosette 1993}

De La Rosette JJ, Hubregtse MR, Meuleman EJ, StolkEngelaar MV, Debruyne FM. Diagnosis and treatment of 409 patients with prostatitis syndromes. Urology 1993;41(4):301-7. [PUBMED: 8470312]

\section{Dellabella 2006}

Dellabella M, Milanese G, Muzzonigro G. Correlation between ultrasound alterations of the preprostatic sphincter and symptoms in patients with chronic prostatitis-chronic pelvic pain syndrome. Journal of Urology 2006;176(1):112-8. [PUBMED: 16753384]

\section{EndNote [Computer program]}

Clarivate Analytics. EndNote. Version 7.5. Clarivate Analytics, 2016.

\section{Franco 2017}

Franco JVA, Tirapegui FI, Turk T, Garrote V, Vietto V. Pharmacological interventions for treating chronic prostatitis/ chronic pelvic pain syndrome. Cochrane Database of Systematic Reviews 2017, Issue 2. [DOI: 10.1002/14651858.CD012552; CD012552]

\section{Gasperi 2017}

Gasperi M, Krieger JN, Forsberg C, Goldberg J, Buchwald D, Afari N. Chronic prostatitis and comorbid non-urological overlapping pain conditions: a co-twin control study. Journal of Psychosomatic Research 2017;102:29-33. [PUBMED: 28992894]

\section{Glenton 2010}

Glenton C, Santesso N, Rosenbaum S, Nilsen ES, Rader T, Ciapponi A, et al. Presenting the results of Cochrane systematic reviews to a consumer audience: a qualitative study. Medical Decision Making 2010;30(5):566-77. [PUBMED: 20643912]

\section{GRADEpro GDT [Computer program]}

GRADE Working Group, McMaster University. GRADEpro GDT. Hamilton (ON): GRADE Working Group, McMaster University, 2015.

\section{Guyatt 2008}

Guyatt GH, Oxman AD, Vist GE, Kunz R, Falck-Ytter Y, Schünemann HJ, et al. GRADE: what is "quality of evidence" and why is it important to clinicians?. BMJ (Clinical Research Ed.) 2008;336(7651):995-8. [DOI: 10.1136/bmj.39490.551019.BE]

\section{Guyatt 2011a}

Guyatt GH, Oxman AD, Kunz R, Brozek J, Alonso-Coello P, Rind $D$, et al. GRADE guidelines 6 . Rating the quality of evidence - imprecision. Journal of Clinical Epidemiology 2011;64(12):1283-93.

\section{Guyatt 2011b}

Guyatt G, Oxman AD, Akl EA, Kunz R, Vist G, Brozek J, et al. GRADE guidelines: 1 . Introduction - GRADE evidence profiles and summary of findings tables. Journal of Clinical Epidemiology 2011;64(4):383-94. [DOI: 10.1016/ j.jclinepi.2010.04.026]

\section{Hetrick 2006}

Hetrick DC, Glazer H, Liu YW, Turner JA, Frest M, Berger RE. Pelvic floor electromyography in men with chronic pelvic pain syndrome: a case-control study. Neurourology and Urodynamics 2006;25(1):46-9. [PUBMED: 16167354]

\section{Higgins 2002}

Higgins JPT, Thompson SG. Quantifying heterogeneity in a meta-analysis. Statistics in Medicine 2002;21(11):1539-58. [DOI: 10.1002/sim.1186]

\section{Higgins 2003}

Higgins JPT, Thompson SG, Deeks JJ, Altman DG. Measuring inconsistency in meta-analyses. BMJ (Clinical Research Ed.) 2003;327(7414):557-60. [DOI: 10.1136/bmj.327.7414.557]

\section{Higgins 2011a}

Higgins JPT, Green S, editor(s). Cochrane Handbook for Systematic Reviews of Interventions. Version 5.1.0 (updated March 2011). The Cochrane Collaboration, 2011. Available from handbook.cochrane.org.

\section{Higgins 2011b}

Higgins JPT, Altman DG, Sterne JAC. Chapter 8: Assessing risk of bias in included studies. In: Higgins JPT, Green S, editor(s). Cochrane Handbook for Systematic Reviews of Interventions. Version 5.1.0 (updated March 2011). The Cochrane Collaboration, 2011. Available from handbook.cochrane.org.

\section{Higgins 2011c}

Higgins JPT, Deeks JJ, Altman DG. Chapter 16: Special topics in statistics. In: Higgins JPT, Green S, editor(s). Cochrane Handbook for Systematic Reviews of Interventions. Version 5.1.0 (updated March 2011). The Cochrane Collaboration, 2011. Available from handbook.cochrane.org.

\section{HIRU 2015}

Health Information Research Unit (HIRU). Evidence-Based Health Informatics. Search strategies for PsycINFO in OVID syntax. Hamilton (ON): McMaster University; 2015. hiru.mcmaster.ca/hiru/HIRU_Hedges_EMBASE_Strategies.aspx (accessed prior to 18 January 2018). 


\section{Hoffmann 2013}

Hoffmann TC, Erueti C, Glasziou PP. Poor description of nonpharmacological interventions: analysis of consecutive sample of randomised trials. BMJ 2013;347:1-10.

\section{Hou 2012}

Hou DS, Long WM, Shen J, Zhao LP, Pang XY, Xu C. Characterisation of the bacterial community in expressed prostatic secretions from patients with chronic prostatitis/ chronic pelvic pain syndrome and infertile men: a preliminary investigation. Asian Journal of Andrology 2012;14(4):566-73. [PUBMED: 22635162]

\section{Hruz 2003}

Hruz P, Danuser H, Studer UE, Hochreiter WW. Noninflammatory chronic pelvic pain syndrome can be caused by bladder neck hypertrophy. European Urology 2003;44(1):106-10; discussion 110. [PUBMED: 12814683]

\section{loannidis 2001}

Ioannidis JP, Lau J. Completeness of safety reporting in randomized trials: an evaluation of 7 medical areas. JAMA 2001;285(4):437-43. [PUBMED: 11242428]

\section{Issa 1996}

Issa Muta M. Transurethral Needle Ablation of the Prostate: Report of Initial United States Clinical Trial. The Journal of Urology 1996;156(2):413-9. [DOI: 10.1016/ S0022-5347(01)65863-4]

\section{Johnston 2010}

Johnston BC, Thorlund K, Schünemann HJ, Xie F, Murad MH, Montori VM, et al. Improving the interpretation of quality of life evidence in meta-analyses: the application of minimal important difference units. Health and Quality of Life Outcomes 2010;8:116.

\section{Kamijo 2001}

Kamijo T, Sato S, Kitamura T. Effect of cernitin pollen-extract on experimental nonbacterial prostatitis in rats. Prostate 2001;49(2):122-31. [PUBMED: 11582591]

\section{Kim 2006}

Kim HW, Roh DH, Yoon SY, Kang SY, Kwon YB, Han HJ, et al. The anti-inflammatory effects of low- and high-frequency electroacupuncture are mediated by peripheral opioids in a mouse air pouch inflammation model. Journal of Alternative and Complementary Medicine (New York, N.Y.) 2006;12(1):39-44. [PUBMED: 16494567]

\section{Kirby 1982}

Kirby RS, Lowe D, Bultitude MI, Shuttleworth KE. Intra-prostatic urinary reflux: an aetiological factor in abacterial prostatitis. British Journal of Urology 1982;54(6):729-31. [PUBMED: 7150931]

\section{Krieger 1996}

Krieger JN, Berger RE, Ross SO, Rothman I, Muller CH. Seminal fluid findings in men with nonbacterial prostatitis and prostatodynia. Journal of Andrology 1996;17(3):310-8. [PUBMED: 8792222]

\section{Krieger 2002}

Krieger JN, Ross SO, Deutsch L, Riley DE. The NIH Consensus concept of chronic prostatitis/chronic pelvic pain syndrome compared with traditional concepts of nonbacterial prostatitis and prostatodynia. Current Urology Reports 2002;3(4):301-6. [PUBMED: 12149161]

\section{Krieger 2004}

Krieger JN. Classification, epidemiology and implications of chronic prostatitis in North America, Europe and Asia. Minerva Urologica e Nefrologica [Italian Journal of Urology and Nephrology] 2004;56(2):99-107. [PUBMED: 15195020]

\section{Krsmanovic 2014}

Krsmanovic A, Tripp DA, Nickel JC, Shoskes DA, Pontari M, Litwin MS, et al. Psychosocial mechanisms of the pain and quality of life relationship for chronic prostatitis/chronic pelvic pain syndrome (CP/CPPS). Journal de l'Association des Urologues du Canada [Canadian Urological Association Journal] 2014;8(11-12):403-8. [PUBMED: 25553153]

\section{Lefebvre 2011}

Lefebvre C, Manheimer E, Glanville J. Chapter 6: Searching for studies. In: Higgins JPT, Green S, editor(s). Cochrane Handbook for Systematic Reviews of Interventions Version 5.1.0 (updated March 2011). The Cochrane Collaboration, 2011. Available from handbook.cochrane.org.

\section{Liberati 2009}

Liberati A, Altman DG, Tetzlaff J, Mulrow C, Gøtzsche PC, Ioannidis JPA, et al. The PRISMA statement for reporting systematic reviews and meta-analyses of studies that evaluate health care interventions: explanation and elaboration. PLoS Medicine 2009;6(7):e1000100. [DOI: 10.1371/ journal.pmed.1000100]

\section{Litwin 1999}

Litwin MS, McNaughton-Collins M, Fowler FJ, Nickel JC, Calhoun EA, Pontari MA, et al. The National Institutes of Health chronic prostatitis symptom index: development and validation of a new outcome measure. Chronic Prostatitis Collaborative Research Network. Journal of Urology 1999;162(2):369-75.

\section{Magri 2010}

Magri V, Wagenlehner F, Perletti G, Schneider S, Marras E, Naber KG, et al. Use of the UPOINT chronic prostatitis/chronic pelvic pain syndrome classification in European patient cohorts: sexual function domain improves correlations. Journal of Urology 2010;184(6):2339-45. [PUBMED: 20952019]

\section{Mehik 2002}

Mehik A, Hellstrom P, Nickel JC, Kilponen A, Leskinen M, Sarpola A, et al. The chronic prostatitis-chronic pelvic pain syndrome can be characterized by prostatic tissue pressure measurements. Journal of Urology 2002;167(1):137-40. [PUBMED: 11743292]

\section{Miller 2002}

Miller LJ, Fischer KA, Goralnick SJ, Litt M, Burleson JA, Albertsen $\mathrm{P}$, et al. Nerve growth factor and chronic prostatitis/ 
chronic pelvic pain syndrome. Urology 2002;59(4):603-8.

[PUBMED: 11927336]

\section{Neal 1994}

Neal DE Jr, Moon TD. Use of terazosin in prostatodynia and validation of a symptom score questionnaire. Urology 1994;43(4):460-5. [PUBMED: 7512296]

\section{Nickel 1999a}

Nickel JC, Nyberg LM, Hennenfent M. Research guidelines for chronic prostatitis: consensus report from the first National Institutes of Health International Prostatitis Collaborative Network. Urology 1999;54(2):229-33. [PUBMED: 10443716]

\section{Nickel 1999b}

Nickel JC, Alexander R, Anderson R, Krieger J, Moon T, Neal D, et al. Prostatitis unplugged? Prostatic massage revisited. Techniques in Urology 1999;5(1):1-7. [PUBMED: 10374787]

\section{Nickel 2003a}

Nickel JC, Alexander RB, Schaeffer AJ, Landis JR, Knauss JS, Propert KJ. Leukocytes and bacteria in men with chronic prostatitis/chronic pelvic pain syndrome compared to asymptomatic controls. Journal of Urology 2003;170(3):818-22. [PUBMED: 12913707]

\section{Nickel 2003b}

Nickel JC, Pontari M, Moon T, Gittelman M, Malek G, Farrington J, et al. A randomized, placebo controlled, multicenter study to evaluate the safety and efficacy of rofecoxib in the treatment of chronic nonbacterial prostatitis. Journal of Urology 2003;169(4):1401-5. [PUBMED: 12629372]

\section{Nickel 2012}

Nickel JC. Prostatitis and related conditions, orchitis and epididymitis. Campbell-Walsh Urology. 10th Edition. Amsterdam: Elsevier, 2012:337-41.

\section{Nyberg 1999}

Nyberg LM, Krieger JN, Nickel JC. National Institutes of Health classification of chronic prostatitis. In: Nickel JC editor(s). Textbook of Prostatitis. London: CRC Press, 1999.

\section{Parker 2013}

Parker SL, Godil SS, Shau DN, Mendenhall SK, McGirt MJ. Assessment of the minimum clinically important difference in pain, disability, and quality of life after anterior cervical discectomy and fusion: clinical article. Journal of Neurosurgery Spine 2013;18(2):154-60.

\section{Pontari 2004}

Pontari MA, Ruggieri MR. Mechanisms in prostatitis/chronic pelvic pain syndrome. Journal of Urology 2004;172(3):839-45. [PUBMED: 15310980]

\section{Pontari 2005}

Pontari MA, McNaughton-Collins M, O'Leary MP, Calhoun EA, Jang T, Kusek JW, et al. A case-control study of risk factors in men with chronic pelvic pain syndrome. BJU International 2005;96(4):559-65. [PUBMED: 16104910]

\section{Propert 2002}

Propert KJ, Alexander RB, Nickel JC, Kusek JW, Litwin MS, Landis JR, et al. Design of a multicenter randomized clinical trial for chronic prostatitis/chronic pelvic pain syndrome. Urology 2002;59(6):870-6. [PUBMED: 12031372]

\section{Propert 2006}

Propert KJ, McNaughton-Collins M, Leiby BE, O'Leary MP, Kusek JW, Litwin MS, et al. A prospective study of symptoms and quality of life in men with chronic prostatitis/chronic pelvic pain syndrome: the National Institutes of Health chronic prostatitis cohort study. Journal of Urology 2006;175(2):619-23.

\section{Rees 2015}

Rees J, Abrahams M, Doble A, Cooper A. Diagnosis and treatment of chronic bacterial prostatitis and chronic prostatitis/chronic pelvic pain syndrome: a consensus guideline. BJU International 2015;116(4):509-25. [PUBMED: 25711488]

\section{RevMan 2014 [Computer program]}

Nordic Cochrane Centre, The Cochrane Collaboration. Review Manager (RevMan). Version 5.3. Copenhagen: Nordic Cochrane Centre, The Cochrane Collaboration, 2014.

\section{Riegel 2014}

Riegel B, Bruenahl CA, Ahyai S, Bingel U, Fisch M, Lowe B. Assessing psychological factors, social aspects and psychiatric co-morbidity associated with chronic prostatitis/chronic pelvic pain syndrome (CP/CPPS) in men - a systematic review. Journal of Psychosomatic Research 2014;77(5):333-50. [PUBMED: 25300538]

\section{Rodriguez 2009}

Rodriguez MA, Afari N, Buchwald DS. Evidence for overlap between urological and nonurological unexplained clinical conditions. Journal of Urology 2009;182(5):2123-31. [PUBMED: 19758633]

\section{Rosen 2011}

Rosen RC, Allen KR, Ni X, Araujo AB. Minimal clinically important differences in the erectile function domain of the International Index of Erectile Function scale. European Urology 2011;60(5):1010-6.

\section{Schaeffer 2002}

Schaeffer AJ, Landis JR, Knauss JS, Propert KJ, Alexander RB, Litwin MS, et al. Demographic and clinical characteristics of men with chronic prostatitis: the National Institutes of Health chronic prostatitis cohort study. Journal of Urology 2002;168(2):593-8. [PUBMED: 12131316]

\section{Schünemann 2011}

Schünemann HJ, Oxman AD, Higgins JPT, Vist GE, Glasziou P, Guyatt GH. Chapter 11: Presenting results and 'Summary of findings' tables. In: Higgins JPT, Green S, editor(s), Cochrane Handbook for Systematic Reviews of Interventions. Version 5.1.0 (updated March 2011). The Cochrane Collaboration, 2011. Available from handbook.cochrane.org. 


\section{Shoskes 2002}

Shoskes DA, Albakri Q, Thomas K, Cook D. Cytokine polymorphisms in men with chronic prostatitis/chronic pelvic pain syndrome: association with diagnosis and treatment response. Journal of Urology 2002;168(1):331-5. [PUBMED: 12050565]

\section{Shoskes 2008a}

Shoskes DA, Berger R, Elmi A, Landis JR, Propert KJ, Zeitlin S. Muscle tenderness in men with chronic prostatitis/chronic pelvic pain syndrome: the chronic prostatitis cohort study. Journal of Urology 2008;179(2):556-60. [PUBMED: 18082223]

\section{Shoskes 2008b}

Shoskes DA, Nickel JC, Rackley RR, Pontari MA. Clinical phenotyping in chronic prostatitis/chronic pelvic pain syndrome and interstitial cystitis: a management strategy for urologic chronic pelvic pain syndromes. Prostate Cancer and Prostatic Diseases 2008;12(2):177-83.

\section{Spaliviero 2010}

Spaliviero M, Strom KH, Gu X, Araki M, Culkin DJ, Wong C. Does Greenlight HPS ${ }^{(\mathrm{TM}}$ ) laser photoselective vaporization prostatectomy affect sexual function?. Journal of Endourology 2010;24(12):2051-7.

\section{Strohmaier 1988}

Strohmaier W, Bichler K, Kiefer M, Lev A. Microwave hyperthermia in chronic prostatitis and prostatodynia preliminary results [Mikrowellenhyperthermie bei chronischer Prostatitis undProstatodynie - vorlaufige Ergebnisse]. Helvetica Chirurgica Acta 1988;55:301-3.

\section{Suskind 2013}

Suskind AM, Berry SH, Ewing BA, Elliott MN, Suttorp MJ, Clemens JQ. The prevalence and overlap of interstitial cystitis/ bladder pain syndrome and chronic prostatitis/chronic pelvic pain syndrome in men: results of the RAND Interstitial Cystitis Epidemiology male study. Journal of Urology 2013;189(1):141-5. [PUBMED: 23164386]

\section{Trinchieri 2007}

Trinchieri A, Magri V, Cariani L, Bonamore R, Restelli A, Garlaschi MC, et al. Prevalence of sexual dysfunction in men with chronic prostatitis/chronic pelvic pain syndrome. Archivio Italiano di Urologia, Andrologia 2007;79(2):67-70. [PUBMED: 17695411]

\section{CHARACTERISTICS OF STUDIES}

Characteristics of included studies [ordered by study ID]

\section{True 1999}

True LD, Berger RE, Rothman I, Ross SO, Krieger JN. Prostate histopathology and the chronic prostatitis/chronic pelvic pain syndrome: a prospective biopsy study. Journal of Urology 1999;162(6):2014-8. [PUBMED: 10569559]

\section{Turner 2003}

Turner JA, Ciol MA, Von Korff M, Berger R. Validity and responsiveness of the national institutes of health chronic prostatitis symptom index. Journal of Urology 2003;169(2):580-3.

\section{Walz 2007}

Walz J, Perrotte P, Hutterer G, Suardi N, Jeldres C, Benard F, et al. Impact of chronic prostatitis-like symptoms on the quality of life in a large group of men. BJU International 2007;100(6):1307-11. [PUBMED: 17941922]

\section{Yang 2003}

Yang CC, Lee JC, Kromm BG, Ciol MA, Berger R. Pain sensitization in male chronic pelvic pain syndrome: why are symptoms so difficult to treat?. Journal of Urology 2003;170(3):823-6; discussion 826-7. [PUBMED: 12913708]

\section{Zhu 2014}

Zhu Y, Wang C, Pang X, Li F, Chen W, Tan W. Antibiotics are not beneficial in the management of category III prostatitis: a meta analysis. Urology Journal 2014;11(2):1377-85. [PUBMED: 24807747]

\section{References to other published versions of this review Franco 2016}

Franco JVA, Tirapegui FI, Garrote V, Vietto V. Interventions for treating chronic prostatitis/chronic pelvic pain syndrome. Cochrane Database of Systematic Reviews 2016, Issue 8. [DOI: 10.1002/14651858.CD012320]

\section{McNaughton 1999}

McNaughton Collins M, MacDonald R, Wilt TJ. Interventions for chronic abacterial prostatitis. Cochrane Database of Systematic Reviews 1999, Issue 4. [DOI: 10.1002/14651858.CD002080]

* Indicates the major publication for the study

Ateya 2006

Methods Study design: parallel group randomised trial.

Study dates: not available.

Setting: academic hospital, outpatient, national.

Country: Egypt. 
Ateya 2006 (Continued)

Participants
Inclusion criteria: consecutive participants who attended outpatient clinic with history or symptoms suggestive of CP (NIH category II and IIIa).

Exclusion criteria: presence of cancer of genitourinary tract; active urinary stone disease or herpes of the genitourinary system; perirectal inflammatory disorders; inflammatory bowel disease; history of pelvic radiation or systemic chemotherapy; history of intravesical chemotherapy; urethral stricture $\leq 12$ F; neurological disease or disorder affecting bladder; and prostate surgery (not including cystoscopy) within the past 3 months.

Sample size: 81.

Age (years): overall 35.3 (SD 9.0).

Baseline NIH-CPSI score: not available.

Sex: men.

Group $1(n=17)$ : participants with chronic bacterial prostatitis who received antibiotics (targeted to culture) and prostatic massage (performed 3 times weekly for 4 weeks). Prostate was massaged from above and lateral to gland, 6 times on each side, by gentle and firm pressure of finger directed downwards and inwards, followed by a few strokes in the middle from above downwards.

Group $2(n=20)$ : participants with chronic bacterial prostatitis who received only antibiotics.

Group $3(n=25)$ : participants with CP/CPPS who received antibiotics empirically and prostatic massage.

Group 4 ( $n=19)$ : participants with CP/CPPS who received antibiotics empirically alone.

Cointerventions: none described.

\section{Prostatitis symptoms}

How measured: NIH-CPSI.

Time points measured: before treatment and end of study.

Time points reported: before and after treatment.

\begin{tabular}{ll}
\hline Funding sources & Not available. \\
\hline Declarations of interest & Not available. \\
\hline Notes & Only group 3 and 4 analysed.
\end{tabular}

\section{Risk of bias}

\begin{tabular}{lll}
\hline Bias & Authors' judgement & Support for judgement \\
\hline $\begin{array}{l}\text { Random sequence genera- } \\
\text { tion (selection bias) }\end{array}$ & Unclear risk & No information available. Study authors did not answer. \\
\hline $\begin{array}{l}\text { Allocation concealment } \\
\text { (selection bias) }\end{array}$ & Unclear risk & No information available. Study authors did not answer. \\
\hline $\begin{array}{l}\text { Blinding of participants } \\
\text { and personnel (perfor- } \\
\text { mance bias) } \\
\begin{array}{l}\text { Subjective outcomes } \\
\text { High risk }\end{array}\end{array}$ & Open label study. \\
\end{tabular}


Ateya 2006 (Continued)

Blinding of outcome as-
sessment (detection bias) $\quad$ High risk $\quad$ Open label study.

All outcomes

\begin{tabular}{lll}
\hline $\begin{array}{l}\text { Incomplete outcome data } \\
\text { (attrition bias) } \\
\text { All outcomes }\end{array}$ & Unclear risk & $\begin{array}{l}\text { No information regarding follow-up for all outcomes. Study authors did not } \\
\text { answer. }\end{array}$ \\
\hline $\begin{array}{l}\text { Selective reporting (re- } \\
\text { porting bias) }\end{array}$ & Unclear risk & $\begin{array}{l}\text { Protocol not available. Wrote to authors to clarify time point of NIH-CPSI score } \\
\text { measurement, but they did not answer. }\end{array}$ \\
\hline Other bias & Unclear risk & $\begin{array}{l}\text { Basal characteristics for each group not presented for variables of interest. We } \\
\text { wrote to authors, but they did not answer. }\end{array}$ \\
\hline
\end{tabular}

Chen 2009

$\begin{array}{ll}\text { Methods } & \text { Study design: parallel group randomised trial. } \\ & \text { Study dates: September } 2004 \text { to December } 2007 \\ & \text { Setting: outpatient. } \\ \text { Country: China. }\end{array}$

Participants

Inclusion criteria: symptoms: swelling of the testes or perineum, pain of lumbosacral region, abnormal urination, dizziness and fatigue, urethral secretions, sexual dysfunctions, etc.; digital examination: increased hardness of prostate, normal size or slightly enlarged, tenderness; laboratory: routine urine tests negative, WBC count of prostatic fluid > 10/HPF, urine culture negative for bacteria.

Exclusion criteria: any advanced systemic disease such as organic disease of the heart, liver and kidney; participants who dropped out because they did not adhere to the treatment.

Sample size: 125 participants.

Age (years):

Group 1: range: $23 \sim 50$, mean: 32.58 (SD 6.61).

Group 2: range: $21^{\sim}$ 49, mean: 34.16 (SD 5.96).

Group 3: range: $25 \sim 52$, mean: 33.19 (SD 7.35).

Baseline NIH-CPSI score: Group 1: mean 22.56 (SD 7.52), Group 2: mean 21.97 (SD 8.65), Group 3: mean 22.89 (SD 7.06).

Sex: men.

Acupuncture at acupoints: BI-18 GanYu (depth 10 $20 \mathrm{~mm}$ ), BI-23, ShenYu (depth 10 $20 \mathrm{~mm}$ ) and BI-54 ZhiBian (depth $40 \mathrm{~mm}$ ) were applied for 5 seconds each; needles removed afterwards. Then acupuncture at acupoints BI-26 GuanYuan, Ren-3 ZhongJi, SP-9 YinLingQuan, SP-6 SanYin Jiao for 5 seconds each (depth $25^{\sim} 40 \mathrm{~mm}$ ); needles were left afterwards and the tails of which were then covered with 2 $\mathrm{cm}$ moxa sticks. Moxa sticks were then ignited. Repeat the moxibustion twice more for each acupoints.

Participants treated once daily. Course of treatment 1 month.

Group $2(n=41)$ : acupuncture alone. 
Chen 2009 (Continued)

Acupuncture procedures same as Group 1. Moxibustion not performed. Participants treated once daily. Course of treatment 1 month.

Group $3(n=42)$ : medical treatment (Western medicine).

Prostat tablet (oral) twice daily: pollen extract.

Course of treatment 1 month.

Cointerventions: participants in all 3 groups underwent 1 course of treatment (1 month).

\begin{tabular}{ll}
\hline Outcomes & Prostatitis symptoms \\
& How measured: NIH-CPSI. \\
& Time points measured: before and after treatment. \\
& Time points reported: before and after treatment (1 month). \\
& Subgroups: none.
\end{tabular}

\begin{tabular}{ll}
\hline Funding sources & Not mentioned. \\
\hline Declarations of interest & Not mentioned. \\
\hline Notes & We extracted data for Groups 1 and 2 to assess the effect of moxibustion. \\
\hline
\end{tabular}

\section{Risk of bias}

\begin{tabular}{|c|c|c|}
\hline Bias & Authors' judgement & Support for judgement \\
\hline $\begin{array}{l}\text { Random sequence genera- } \\
\text { tion (selection bias) }\end{array}$ & Low risk & $\begin{array}{l}\text { Quote: '(Participants) were randomized by referring their date of admission to } \\
\text { a random number table' (p. 275). }\end{array}$ \\
\hline $\begin{array}{l}\text { Allocation concealment } \\
\text { (selection bias) }\end{array}$ & Unclear risk & Allocation concealment not described. \\
\hline $\begin{array}{l}\text { Blinding of participants } \\
\text { and personnel (perfor- } \\
\text { mance bias) } \\
\text { Subjective outcomes }\end{array}$ & High risk & $\begin{array}{l}\text { Masking of participants and personnel not described. However, considerable } \\
\text { visible difference between the } 3 \text { interventions. Therefore, masking unlikely. }\end{array}$ \\
\hline $\begin{array}{l}\text { Blinding of outcome as- } \\
\text { sessment (detection bias) } \\
\text { All outcomes }\end{array}$ & High risk & All outcomes (participant-reported outcomes): blinding unlikely (see above). \\
\hline $\begin{array}{l}\text { Incomplete outcome data } \\
\text { (attrition bias) } \\
\text { All outcomes }\end{array}$ & Low risk & All outcomes: outcome data available for all randomised participants. \\
\hline $\begin{array}{l}\text { Selective reporting (re- } \\
\text { porting bias) }\end{array}$ & Unclear risk & $\begin{array}{l}\text { Unclear whether there was selective outcome reporting. Protocol not avail- } \\
\text { able. }\end{array}$ \\
\hline Other bias & Low risk & No other sources of bias identified. \\
\hline
\end{tabular}

Fang 2005

Methods Study design: parallel group randomised trial.


Fang 2005 (Continued)

Study dates: April 2002 to March 2004.

Setting: outpatient.

Country: China.

Participants

Inclusion criteria: participants undertook 'Meares-Stamey' test, the initial urine, midstream urine, EPS, and post-prostatic massage urine routine test and bacterial culture and then diagnosed with CP/CPPS. Classified as type IIIa or type IIIb CP by NIH-CPSI classification.

Exclusion criteria: acute prostatitis or chronic bacterial prostatitis; could not attend hospital regularly or had poor compliance; had undertaken repeated sessions of prostate radiofrequency, thermotherapy or other physical therapies; had undertaken repeated sessions of intraprostate injection of antibiotics.

Sample size: 112 .

Age (years):

Group 1: range $22 \sim 47$, mean: 33.6 .

Group 2: range 24 52, mean: 34.

Baseline NIH-CPSI score: not available.

Sex: men.

Interventions

Group $1(\mathrm{n}=56)$ : He-Ne laser.

Treated using He-Ne laser equipment every other day. Optic fibre inserted from urethra and ending located at prostatic urethra.

Output power of optic fibre ending: $10 \mathrm{~mW}$.

Energy of irradiation: $18 \mathrm{~J}$ each time.

1 course of treatment: 10 times of radiation ( 2 sessions weekly).

Discontinued all other treatments, except for some participants where short-term sulpha-drugs were administered temporarily to prevent infection.

Group $2(n=56)$ : drug combination.

Routine therapy for $\mathrm{CP}$ :

Antibiotics: compound sulfamethoxazole, 2 tablets, orally, twice daily, 60 days of fluoroquinolones such as levofloxacin $0.2 \mathrm{~g}$, twice daily, 14 days.

Adjuvant drugs: pollen drugs such as Prostat; Chinese patent drugs such as salvianolic acid $B$ and saponins of panax notoginseng mixture (SalB/PNS).

Physical therapy: hot water bath.

Changes of lifestyle.

Cointerventions: not available.

\section{Outcomes Prostatitis symptoms}

How measured: changes in NIH-CPSI.

Time points measured: before and after treatment.

Time points reported: after treatment.

Subgroups: none. 
Fang 2005 (Continued)

\section{Adverse events}

How measured: narratively.

\begin{tabular}{ll}
\hline Funding sources & Not mentioned. \\
\hline Declarations of interest & Not mentioned. \\
\hline Notes & None. \\
\hline
\end{tabular}

\section{Risk of bias}

\begin{tabular}{|c|c|c|}
\hline Bias & Authors' judgement & Support for judgement \\
\hline $\begin{array}{l}\text { Random sequence genera- } \\
\text { tion (selection bias) }\end{array}$ & Unclear risk & $\begin{array}{l}\text { Quote: ' } 112 \text { patients were randomly divided into laser therapy group and com- } \\
\text { bination drug therapy group' (in Chinese); however, unclear what method } \\
\text { used. }\end{array}$ \\
\hline $\begin{array}{l}\text { Allocation concealment } \\
\text { (selection bias) }\end{array}$ & Unclear risk & Allocation concealment not described. \\
\hline $\begin{array}{l}\text { Blinding of participants } \\
\text { and personnel (perfor- } \\
\text { mance bias) } \\
\text { Subjective outcomes }\end{array}$ & High risk & $\begin{array}{l}\text { Masking of participants and personnel not described. Considering the visibly } \\
\text { different interventions, blinding was unlikely. }\end{array}$ \\
\hline $\begin{array}{l}\text { Blinding of outcome as- } \\
\text { sessment (detection bias) } \\
\text { All outcomes }\end{array}$ & High risk & All outcomes (participant-reported outcomes): blinding unlikely (see above). \\
\hline $\begin{array}{l}\text { Incomplete outcome data } \\
\text { (attrition bias) } \\
\text { All outcomes }\end{array}$ & Low risk & All outcomes: outcome data available for all randomised participants. \\
\hline $\begin{array}{l}\text { Selective reporting (re- } \\
\text { porting bias) }\end{array}$ & Unclear risk & $\begin{array}{l}\text { Unclear whether there was selective outcome reporting (protocol not avail- } \\
\text { able). }\end{array}$ \\
\hline Other bias & Low risk & No other sources of bias identified. \\
\hline
\end{tabular}

\section{Fitzgerald 2013}

\begin{tabular}{l} 
Methods \\
Study design: parallel group randomised trial. \\
Study dates: not available. \\
Setting: academic hospital, outpatient. \\
Country: US. \\
\hline $\begin{array}{l}\text { Inclusion criteria: aged } \geq 18 \text { years with clinical diagnosis of CP/CPPS in the opinion of the investigator, } \\
\text { NIH-CPSI score } \geq 15, \text { with symptoms of discomfort or pain in pelvic region for } \geq 3 \text {-month period within }\end{array}$ \\
the last 6 months (<3 years). \\
$\begin{array}{l}\text { Additionally: presence of tenderness/pain to palpation found by physician in } 1 \text { of the pelvic floor mus- } \\
\text { apist at screening visit } 2 \text {. Participants must have previously undergone at least } 1 \text { course of another } \\
\text { form of treatment. }\end{array}$
\end{tabular}


Exclusion criteria: presence of painful scars on lower abdominal wall that, according to healthcare personnel, were unlikely to respond to physical therapy; positive urine culture (>100,000 CFU/mL); unable to tolerate insertion of 1 rectal examining finger; prior course of physical therapy (biofeedback, electrical stimulation or pelvic floor exercises not exclusionary) or had neurological disorder that affected bladder or neuromuscular function in opinion of investigator; presence of active urethral or ureteral calculi, urethral diverticulum, history of pelvic radiation therapy, tuberculous cystitis, bladder cancer, carcinoma in situ, prostate cancer or urethral cancer; any severe, debilitating or urgent concurrent medical condition or a potentially significant pelvic pathology or abnormalities on examination or prior imaging, pelvic mass, etc.

For men: treatment for bacterial prostatitis, unevaluated suspicious prostate examination requiring further evaluation; unilateral orchialgia without other pelvic symptoms or urethral stricture.

Sample size: 47 in total, 23 men of which 21 had CP/CPPS.

Age (years): Group 1: men and women: 44.9 (SD 14.0); Group 2: men and women: 41.1 (SD 11.4).

Baseline NIH-CPSI score: Group 1: 25.8 (SD 5.7); Group 2: 33.5 (SD 4.3).

Sex: men and women. Data extracted for men with CP/CPPS.

Interventions

Treatments provided by certified and trained physical therapists.

Group 1 ( $n=10): 10 \times 1$-hour sessions of global therapeutic Western massage: 'Techniques used include effleurage, petrissage, friction, tapotement, vibration and kneading. These techniques were applied in upper and lower limbs, trunk, buttocks, abdomen, head and neck each for prescribed time periods (e.g. 10 min massage to head and neck). Patients randomized to GTM [global therapeutic massage] were not provided with a home exercise program.'

Group 2 ( $n=11$ ): $10 \times 1$-hour sessions of myofascial physical therapy involved 'connective tissue manipulation of the abdominal wall, back, buttocks and thighs that clinically were found to contain connective tissue abnormalities in the prone and supine position. Other indications were double voiding and squatting (as home exercises). In the later sessions, trans-rectal manipulation was involved.'

Cointerventions: not described.

Outcomes

\section{Prostatitis symptoms}

How measured: NIH-CPSI.

Time points measured: baseline and 12 weeks.

Time points reported: baseline and 12 weeks.

\section{Quality of life}

How measured: Medical Outcomes Study Short Form 12 Health Status Questionnaire.

Time points measured: baseline and 12 weeks.

Time points reported: baseline and 12 weeks.

\section{Sexual dysfunction}

How measured: Sexual Health Inventory for Men.

Time points measured: baseline and 12 weeks.

Time points reported: baseline and 12 weeks.

\section{Adverse events}

How measured: narratively. However, this outcome was not reported separately for men (we contacted study authors). 
Fitzgerald 2013 (Continued)

Funding sources National Institute of Diabetes and Digestive and Kidney Diseases.
Financial interest or other relationship (or both) with Astellas, GSK, Boston Scientific and Bioness Inc. Pfizer, Medtronic, Boston Scientific, Indevus, Allergan, Trillium Therapeutics, Bristol-Myers Squibb, Sanofi-Aventis, Merck and Tengion.
Notes Clinical Trial record: NCT00434343.

Study included men and women with CP/CPPS and interstitial cystitis/bladder pain syndrome. Data extracted for this study only included the subgroup of men with CP/CPPS.

\section{Risk of bias}

\begin{tabular}{|c|c|c|}
\hline Bias & Authors' judgement & Support for judgement \\
\hline $\begin{array}{l}\text { Random sequence genera- } \\
\text { tion (selection bias) }\end{array}$ & Low risk & $\begin{array}{l}\text { Quote: 'were randomly assigned in equal proportions [...] via a prespecified } \\
\text { sequence distributed in a series of sealed envelopes.' }\end{array}$ \\
\hline $\begin{array}{l}\text { Allocation concealment } \\
\text { (selection bias) }\end{array}$ & Low risk & $\begin{array}{l}\text { Quote: 'were randomly assigned in equal proportions [...] via a prespecified } \\
\text { sequence distributed in a series of sealed envelopes.' }\end{array}$ \\
\hline $\begin{array}{l}\text { Blinding of participants } \\
\text { and personnel (perfor- } \\
\text { mance bias) } \\
\text { Subjective outcomes }\end{array}$ & High risk & $\begin{array}{l}\text { Personnel not blinded. Quote: 'It was not possible to blind study participants } \\
\text { to treatment assignment as more than } 90 \% \text { were aware of their treatment } \\
\text { group when queried at the end of the study.' }\end{array}$ \\
\hline $\begin{array}{l}\text { Blinding of outcome as- } \\
\text { sessment (detection bias) } \\
\text { All outcomes }\end{array}$ & High risk & Blinding of participants not possible (see quote above). \\
\hline $\begin{array}{l}\text { Incomplete outcome data } \\
\text { (attrition bias) } \\
\text { All outcomes }\end{array}$ & Low risk & $\begin{array}{l}43 \text { participants randomised, } 21 \text { men with CP/CPPS ( } 10 \text { in Group } 1 \text { and } 11 \text { in } \\
\text { group 2). Outcome data available for } 9 \text { participants in Group } 1 \text { and } 10 \text { partici- } \\
\text { pants in Group } 2 \text {. Attrition was low and balanced between groups. }\end{array}$ \\
\hline $\begin{array}{l}\text { Selective reporting (re- } \\
\text { porting bias) }\end{array}$ & Low risk & $\begin{array}{l}\text { ClinicalTrial.gov record reported outcomes of feasibility and the study report } \\
\text { included clinical outcomes. However, no evidence of lack of reporting of clini- } \\
\text { cal outcomes. }\end{array}$ \\
\hline Other bias & High risk & $\begin{array}{l}\text { Baseline differences between NIH-CPSI scores: Group 1: } 25.8 \text { (SD 5.7); Group 2: } \\
\text { 33.5 (SD 4.3). }\end{array}$ \\
\hline
\end{tabular}

Gallo 2014

\begin{tabular}{ll} 
Methods & Study design: parallel group randomised trial. \\
Study dates: January 2012 to March 2013. \\
Setting: outpatient. \\
Country: Italy. \\
\hline Inclusion criteria: diagnosis of category IIIa or IIIb CPPS, aged 20-50 years; score $>1$ in pain domain of \\
NIH-CPSI; duration of symptoms > 3 months and < 12 months; presence of at least 1 risk factor in clini- \\
cal history. \\
Exclusion criteria: category I or II CPPS assessed after lower urinary tract localisation studies; previ- \\
ous urinary tract infection within last year; consumption of drugs that could modify lower urinary tract
\end{tabular}


Gallo 2014 (Continued)

function; severe gastric problems, coagulation problems, renal or hepatic (or both) failure contraindicating consumption of NSAIDs.

Sample size: 100 .

Age (years): Group 1: mean 34.2 (SD 8); Group 2: mean 33.2 (SD 7.8).

Baseline NIH-CPSI score: Group 1: mean 21.9 (SD 6.9); Group 2: mean 22.1 (SD 6.4).

Sex: men.

Interventions

Group $1(n=50)$ : control. Invited to follow same diet, sexual behaviours and lifestyle as previous months.

Group 2 ( $n=50)$ : lifestyle interventions. Authors individually discussed the risk factors detected at their history by the refilled questionnaire. Then informed participants that such risk factors were potential causes of their disease symptoms and it was strongly recommended to avoid them. Distributed a vademecum with 13 rules relating to diet, sexual habits and lifestyle. At 2 nd visit, participants screened for adverse events and excluded from analysis if they had not followed the rules.

Cointerventions: after randomisation, participants given nimesulide $100 \mathrm{mg}$ twice daily for 1 week.

Prostatitis symptoms
How measured: NIH-CPSI score.
Time points measured: baseline and 3 months after intervention.
Time points reported: baseline and 3 months after intervention (graphically).
6-point reduction in the total symptom score after treatment was a criterion of response to treatment.
Reported as dichotomous outcome.

\begin{tabular}{ll}
\hline Funding sources & Not available. \\
\hline Declarations of interest & Authors declared no conflict of interest. \\
\hline Notes & Clinical trial record: ACTRN12611000441910. \\
& We wrote to study authors for clarification; however, Dr Luigi Gallo provided no further information. \\
\hline
\end{tabular}

\section{Risk of bias}

Bias Authors' judgement Support for judgement

Random sequence genera- Unclear risk tion (selection bias)

Quote: 'a stratified randomization system, recruited patients were assigned into two homogeneous and equal groups according to the baseline value of $\mathrm{NIH}$ CPSI, patients age, duration of symptoms and a number of detected risk factors.'

Clinical trial records described 'adaptive randomization.'

No additional information provided on how it was generated.

\begin{tabular}{lll}
\hline $\begin{array}{l}\text { Allocation concealment } \\
\text { (selection bias) }\end{array}$ & High risk & Allocation not concealed (clinical trial record). \\
\hline $\begin{array}{l}\text { Blinding of participants } \\
\text { and personnel (perfor- } \\
\text { mance bias) } \\
\text { Subjective outcomes }\end{array}$ & High risk & Open label study (clinical trial record). \\
\hline
\end{tabular}


Gallo 2014 (Continued)

Blinding of outcome as- High risk Open label study (clinical trial record). sessment (detection bias) All outcomes

$\begin{array}{ll}\begin{array}{l}\text { Incomplete outcome data } \\ \text { (attrition bias) }\end{array} & \text { High risk } \\ \text { All outcomes } & \begin{array}{l}\text { 11/50 participants in Group } 2 \text { excluded from analysis of all outcomes due to } \\ \text { poor adherence to protocol; no information regarding availability of outcome } \\ \text { data in Group } 1 .\end{array}\end{array}$

\begin{tabular}{|c|c|c|}
\hline $\begin{array}{l}\text { Selective reporting (re- } \\
\text { porting bias) }\end{array}$ & High risk & $\begin{array}{l}\text { Clinical trial registry described the reported outcomes; however, prespecified } \\
\text { subscores only presented graphically. }\end{array}$ \\
\hline
\end{tabular}

\begin{tabular}{|c|c|c|}
\hline Other bias & Unclear risk & $\begin{array}{l}\text { Clinical trial registry described the use of Serenoa repens in both groups but in } \\
\text { study report this was not mentioned. }\end{array}$ \\
\hline
\end{tabular}

\title{
Gao 2012
}

$\begin{array}{ll}\text { Methods } & \text { Study design: parallel group randomised controlled trial. } \\ & \text { Study dates: January } 2008 \text { to March } 2009 . \\ & \text { Setting: academic hospital. } \\ & \text { Country: China. }\end{array}$

\begin{abstract}
Participants
Inclusion criteria: men aged $\geq 18$ years with symptoms of CP within previous 3 months and pain or discomfort in pelvic region for $\geq 6$ weeks and total score of $\geq 12$ on NIH-CPSI.

Exclusion criteria: urinary tract infection; bacteriuria; history of urethritis with discharge 4 weeks before study entry; history of epididymitis or sexually transmitted infection; residual volume $>50 \mathrm{~mL}$ resulting from bladder outlet obstruction by urodynamic evaluation; indication for, or history of, prostate surgery, including prostate biopsy; history of urogenital cancer; neurological disease affecting bladder; treatment with phytotherapeutic agents, alpha-blockers or antimicrobial substances with prostatic penetration 4 weeks before study entry; treatment with agents influencing intraprostatic hormone metabolism 6 months before study entry and unmarried or with no children.
\end{abstract}

Sample size: 159 (105 included type III CP/CPPS).

Age (mean, years): Group 1: 35.9 for type IIla, 36.7 for type IIlb; Group 2: 36.5 for type IIla, 35.6 for type IIIb; Group 3: 34.7 for type IIla, 39.3 for type IIIb.

Baseline NIH-CPSI score (mean): Group 1: 20.9 for type IIIa, 20.2 for type IIIb; Group 2: 20.0 for type IIIa, 21.1 for type IIIb; Group 3: 22.4 for type IIIa, 21.7 for type IIIb.

Sex: men.

Group 2 ( $n=32$ ): 60-min treatment with TRFH (ZRL-II-A cavity intervention treatment instrument (Shanghai Songhang Industry, Co. Ltd., Shanghai, China), temperature $40-43^{\circ} \mathrm{C}$ ) every day for 5 days.

Group $3(n=43):$ TRFH combined with tamsulosin + clarithromycin.

Outcomes Prostatitis symptoms

How measured: NIH-CPSI.

Time points measured: pretreatment and 6 weeks.

Time points reported: pretreatment and 6 weeks. 
Gao 2012 (Continued)

Subgroups: no subgroup relevant to the review.

\begin{tabular}{ll}
\hline Funding sources & Not stated. \\
\hline Declarations of interest & Not stated. \\
\hline Notes & $\begin{array}{l}\text { Other outcomes included changes in malondialdehyde, superoxide dismutase, nitrogen monoxide and } \\
\text { zinc (for the study of inflammation). }\end{array}$ \\
\hline
\end{tabular}

\section{Risk of bias}

\begin{tabular}{|c|c|c|}
\hline Bias & Authors' judgement & Support for judgement \\
\hline $\begin{array}{l}\text { Random sequence genera- } \\
\text { tion (selection bias) }\end{array}$ & Unclear risk & $\begin{array}{l}\text { Quote: 'randomly divided into } 3 \text { treatment groups based on the order of their } \\
\text { arrival.' No information available. Wrote to authors. }\end{array}$ \\
\hline $\begin{array}{l}\text { Allocation concealment } \\
\text { (selection bias) }\end{array}$ & Unclear risk & No information available. Wrote to authors. \\
\hline $\begin{array}{l}\text { Blinding of participants } \\
\text { and personnel (perfor- } \\
\text { mance bias) } \\
\text { Subjective outcomes }\end{array}$ & High risk & $\begin{array}{l}\text { Blinding was not likely for the comparison of Group } 1 \text { with Groups } 2 \text { and } 3 . \\
\text { Blinding not specified for any comparison. }\end{array}$ \\
\hline $\begin{array}{l}\text { Blinding of outcome as- } \\
\text { sessment (detection bias) } \\
\text { All outcomes }\end{array}$ & High risk & $\begin{array}{l}\text { Blinding was not likely for the comparison of Group } 1 \text { with Groups } 2 \text { and } 3 . \\
\text { Blinding not specified for any comparison. Self-reported outcome. }\end{array}$ \\
\hline $\begin{array}{l}\text { Incomplete outcome data } \\
\text { (attrition bias) } \\
\text { All outcomes }\end{array}$ & Low risk & All outcomes: outcome data available for all randomised participants. \\
\hline $\begin{array}{l}\text { Selective reporting (re- } \\
\text { porting bias) }\end{array}$ & Unclear risk & Protocol not available. \\
\hline Other bias & Low risk & No other sources of bias detected. \\
\hline
\end{tabular}

Giubilei 2007

\begin{tabular}{|c|c|}
\hline \multirow[t]{4}{*}{ Methods } & Study design: parallel group randomised trial. \\
\hline & Study dates: $2002-2004$. \\
\hline & Setting: academic hospital. \\
\hline & Country: Italy. \\
\hline \multirow[t]{3}{*}{ Participants } & $\begin{array}{l}\text { Inclusion criteria: sedentary men with type III CP/CPPS defined according criteria of the National Insti- } \\
\text { tutes of Health International Prostatitis Collaborative Network report, without any medical or psycho- } \\
\text { logical contraindication for moderate intensity physical exercise; 'who reported decreased confidence } \\
\text { in conventional treatments.' }\end{array}$ \\
\hline & $\begin{array}{l}\text { Exclusion criteria: aged > } 50 \text { years and those defined by the Prostatitis Collaborative Network (see } \\
\text { Propert 2002). Reasons for non-participation were 'lack of interest,' 'lack of time' and 'lack of confi- } \\
\text { dence' to engage physical activity. }\end{array}$ \\
\hline & Sample size: 103. \\
\hline
\end{tabular}


Giubilei 2007 (Continued)

Age (years): Group 1: 37.58 (SD 7.80) years; Group 2: 35.88 (SD 8.45).

Baseline NIH-CPSI score: Group 1: 23.46 (SD 7.49); Group 2: 23.55 (SD 5.82).

Sex: men.

Interventions

Group 1 ( $n=48$ ): aerobic exercise group. 18-week walking programme, 3 times weekly, '1) warm up and cool-down regimen of slow paced walking, 2) specific postural muscle and isometric strengthening exercises, 3) $40 \mathrm{~min}$ of fast-paced walking on in-outdoor track. During the fast-paced walking, subjects exercised at $70 / 80 \%$ of the predicted maximum heart rate for their age.'

Group 2 ( $n=49$ ): placebo/flexibility and motion exercise programme; with same period and frequency of Group 1, maintaining their heart rate under 100 beats per min.

Outcomes Prostatitis symptoms

How measured: Italian NIH-CPSI.

Time points measured: 1 week after screening, and 6 and 18 weeks after randomisation.

Time points reported: baseline, 6 and 18 weeks after randomisation.

\section{Anxiety and depression}

How measured: State Anxiety Inventory-Y score, self-administrated questionnaire.

Time points measured: 1 week after screening, and 6 and 18 weeks after randomisation.

Time points reported: baseline, 6 and 18 weeks after randomisation.

\section{Depression}

How measured: Beck Depression Inventory score, self-administrated questionnaire.

Time points measured: 1 week after screening, and 6 and 18 weeks after randomisation.

Time points reported: baseline, 6 and 18 weeks after randomisation.

\begin{tabular}{ll}
\hline Funding sources & Not available. \\
\hline Declarations of interest & Not available. \\
\hline Notes & None.
\end{tabular}

\section{Risk of bias}

\begin{tabular}{lll}
\hline Bias & Authors' judgement & Support for judgement \\
\hline $\begin{array}{l}\text { Random sequence genera- } \\
\text { tion (selection bias) }\end{array}$ & Unclear risk & Not specified in report. \\
\hline $\begin{array}{l}\text { Allocation concealment } \\
\text { (selection bias) }\end{array}$ & Unclear risk & Not specified in report. \\
\hline $\begin{array}{l}\text { Blinding of participants } \\
\text { and personnel (perfor- } \\
\text { mance bias) }\end{array}$ & High risk & $\begin{array}{l}\text { Quote: 'Subjects were not specifically informed about the 2 treatments and } \\
\text { they only knew about physical therapy in the protocol, so that they remained } \\
\text { blinded to the randomization group.' However, unclear how this blinding was } \\
\text { carried out throughout the protocol (questionnaire assessing blinding men- } \\
\text { tioned but not reported in results) and blinding of personnel not specified. } \\
\text { Masking unlikely. }\end{array}$ \\
\hline
\end{tabular}


Giubilei 2007 (Continued)

Blinding of outcome as- High risk Subjective symptoms self-reported by participants. Masking unlikely (see sessment (detection bias) above).

All outcomes

Incomplete outcome data High risk

(attrition bias)

All outcomes
103 participants randomised to Group $1(n=52)$ and Group $2(n=51)$. Outcome data (all outcomes) available for 41 participants in Group 1 and 44 participants in Group 2 at 6 weeks. At 18 weeks, outcome data (all outcomes) available for 36 participants in Group 1 and 40 participants in Group 2.

Selective reporting (re- Unclear risk $\quad$ Protocol not available.
porting bias)
porting bias)

Other bias Low risk No other sources of bias identified.

\section{Kabay 2009}

Methods

Study design: parallel group randomised trial.

Study dates: May 2006 to March 2008.

Setting: academic hospital, outpatient.

Country: Turkey.

\section{Participants}

Inclusion criteria: participants with chronic, therapy-resistant pelvic pain category IIIb defined as complaints of pain for $\geq 6$ months in bladder, groin, genitals or lower abdomen and/or perineal or perianal pain without any obvious abnormalities on urological examination and prior surgical intervention.

Exclusion criteria: chronic bacterial prostatitis or category IIla CP/CPPS, aged $<18$ years, symptoms existing for $<6$ months, active or recurrent urinary tract infection, bladder or kidney stone, bacterial prostatitis, sexually transmitted disease, bladder and prostate malignancy, interstitial cystitis and severe systemic diseases.

Sample size: 89.

Age (years): Group 1: 37.9 (SD 7.6); Group 2: 38.8 (SD 7.2).

Baseline NIH-CPSI score: Group 1: 23.6 (SD 6.3); Group 2: 22.8 (SD 5.4).

Sex: men.

Interventions

Group $1(n=45)$ : PTNS applied unilaterally with 26-gauge stainless steel needles inserted $5 \mathrm{~cm}$ cephalad from medial malleolus and posterior to edge of tibia with ground neutral electrode placed on same leg near arch of foot; both connected to a stimulator at $200 \mu$ seconds with pulse rate $20 \mathrm{~Hz}$ (Medtronic Key Point Net, Medtronic); total of 12 weeks of 30-min sessions.

Group 2 ( $n=44)$ : same electrode procedure for PTNS but stimulator not connected.

Cointerventions: analgesics stopped for 2 weeks prior to trial and physiotherapy or electrotherapy restricted for at least 3 months prior to the PTNS treatment.

Outcomes

\section{Prostatitis symptoms}

How measured: NIH-CPSI score and subscores.

Time points measured: baseline and 12 weeks.

Time points reported: baseline and 12 weeks. 
Kabay 2009 (Continued)

\begin{tabular}{ll} 
Funding sources & Not available. \\
\hline Declarations of interest & Not available. \\
\hline Notes & None.
\end{tabular}

\section{Risk of bias}

\begin{tabular}{lll}
\hline Bias & Authors' judgement & Support for judgement \\
\hline $\begin{array}{l}\text { Random sequence genera- } \\
\text { tion (selection bias) }\end{array}$ & Unclear risk & No information available. Wrote to study authors. \\
\hline $\begin{array}{l}\text { Allocation concealment } \\
\text { (selection bias) }\end{array}$ & Unclear risk & No information available. Wrote to study authors. \\
\hline $\begin{array}{l}\text { Blinding of participants } \\
\text { and personnel (perfor- } \\
\text { mance bias) } \\
\text { Subjective outcomes }\end{array}$ & High risk & Sham treatment had stimulation disconnected, therefore blinding unlikely. \\
\hline $\begin{array}{l}\text { Blinding of outcome as- } \\
\text { sessment (detection bias) } \\
\text { All outcomes }\end{array}$ & High risk & Sham treatment had stimulation disconnected, therefore blinding unlikely. \\
\hline $\begin{array}{l}\text { Incomplete outcome data } \\
\begin{array}{l}\text { (attrition bias) } \\
\text { All outcomes }\end{array}\end{array}$ & Low risk & All outcomes: outcome data available for all randomised participants. \\
\hline $\begin{array}{l}\text { Selective reporting (re- } \\
\text { porting bias) }\end{array}$ & Unclear risk & Protocol not available. \\
\hline \begin{tabular}{l} 
Other bias \\
\hline
\end{tabular} & Low risk & No other sources of bias detected. \\
\hline
\end{tabular}

Kaikai 2014

Methods
Study design: parallel group randomised trial.
Study dates: September 2013 to March 2014.
Setting: outpatient.
Country: China.

Participants

Inclusion criteria: aged $\geq 18$; years; participants had pain or discomfort of the lower abdomen, pelvis, lumbosacral region, penis, scrotum or perineum; participants had clinical manifestations such as frequent urination, urgent urination, urinary retention or sexual dysfunction; prostatic fluid: WBC and lecithin bodies count normal or abnormal, bacteria culture negative; EPS and urine culture negative; course of disease $\geq 3$ months; NIH-CPSI pain subscore and urination subscore $\geq 10$; antibiotics treatment ineffective.

Exclusion criteria: congenital urinary tract malformation, injury to urethra or history of transurethral surgery, history of infection of urinary system within 3 months, history of tumour or tuberculosis of urinary system or pelvis, neurological disorders, acute or chronic bacterial prostatitis, severe diseases of cardiovascular or endocrine system, history of taking alpha-blockers or alpha-adrenergic drugs.

Sample size: 105 . 
Kaikai 2014 (Continued)

Age (years): overall: $18^{\sim} 55$, mean: 32.2 .

Baseline NIH-CPSI score: Group 1: mean 25.9 (SD 2.43); Group 2: mean 26.17 (SD 2.38), Group 3: mean 26.85 (SD 2.15).

Sex: men.

Interventions

Group A $(n=35)$ : non-intrusive ultrasound + integrated Chinese-Western medications.

Output frequency: $1.79 \mathrm{MHz}$.

Output power: $3.15 \mathrm{~W} / \mathrm{cm}^{2}$.

Duration: $20 \mathrm{~min}$.

Administration of treatment every 3 days (total 7 times).

Group B ( $=35)$ : integrated Chinese-Western medications only.

QianLieShuTong capsule, orally, 3 times daily, 3 capsules each time.

Tamsulosin hydrochloride delayed-release capsule, $0.2 \mathrm{mg}$, orally, once daily.

Taken for 1 month.

Group C ( $n=35)$ : non-intrusive ultrasound only.

\section{Outcomes}

\section{Prostatitis symptoms}

How measured: NIH-CPSI global and subscore.

Time points measured: before and after treatment.

Time points reported: before and after treatment.

Subgroups: none.

\section{Adverse events}

How measured: narratively.

\begin{tabular}{ll}
\hline Funding sources & Not mentioned. \\
\hline Declarations of interest & Not mentioned. \\
\hline Notes & None. \\
\hline
\end{tabular}

\section{Risk of bias}

\begin{tabular}{|c|c|c|}
\hline Bias & Authors' judgement & Support for judgement \\
\hline $\begin{array}{l}\text { Random sequence genera- } \\
\text { tion (selection bias) }\end{array}$ & Unclear risk & $\begin{array}{l}\text { Quote: ' } 105 \text { patients were randomly assigned to } 3 \text { groups, } 35 \text { patients each.' } \\
\text { However, method for randomization not described. }\end{array}$ \\
\hline $\begin{array}{l}\text { Allocation concealment } \\
\text { (selection bias) }\end{array}$ & Unclear risk & Allocation concealment not described. \\
\hline $\begin{array}{l}\text { Blinding of participants } \\
\text { and personnel (perfor- } \\
\text { mance bias) } \\
\text { Subjective outcomes }\end{array}$ & High risk & $\begin{array}{l}\text { Masking of participants and personnel not described. However, considerable } \\
\text { visible difference between the } 3 \text { interventions. Therefore, masking unlikely. }\end{array}$ \\
\hline
\end{tabular}


Kaikai 2014 (Continued)

Blinding of outcome as- High risk All outcomes (participant-reported outcomes): blinding unlikely (see above). sessment (detection bias)

All outcomes

Incomplete outcome data Low risk All outcomes: outcome data available for all randomised participants.
(attrition bias)

(attrition bias)

All outcomes

Selective reporting (re- $\quad$ Unclear risk $\quad$ Protocol not available.
porting bias)

Other bias Low risk No other sources of bias detected

Kastner 2004

Methods $\quad$ Study design: parallel group randomised trial.

Study dates: not available.

Setting: academic hospitals, outpatient.

Country: Chile, Switzerland, UK.

Participants Inclusion criteria: men with a diagnosis consistent with NIH IIla or IIIb CP/CPPS symptomatic for $\geq 3$ of preceding 6 months, in whom standard treatments (antibiotics or anti-inflammatory agents, or both) had failed, and who had scored $>8$ points on the NIH-CPSI pain score. A 'treatment criteria' included patients not using alpha-blockers, antiandrogens and finasteride 60 days before enrolment.

Exclusion criteria: diagnosis of urinary tract infection, any illness such as prostate or bladder cancer that could affect urogenital function, or previous surgical intervention to prostate.

Sample size: 42 included; 3 were excluded after randomisation ( 2 were excluded for not meeting the patient's characteristics inclusion criteria and one for not meeting the 'treatment criteria').

Age (years): Group 1: 61.7 (SD 9.4); Group 2: 58.3 (SD 14.4).

Baseline NIH-CPSI score: Group 1: 11.5 (SD 2.8); Group 2: 10.9 (SD 1.8).

Sex: men.

Interventions

Both groups received transurethral microwave thermotherapy through catheter connected to a Targis System.

Group $1(n=21)$ : estimated peak intraprostatic temperatures of $55^{\circ} \mathrm{C}$.

Group 2 ( $n=18)$ : estimated peak intraprostatic temperatures of $70^{\circ} \mathrm{C}$.

Cointerventions: alpha-blockers, antiandrogens and finasteride prohibited for 60 days before enrolment; after treatment, it was left to physician discretion and recorded at follow-up.

Outcomes

\section{Prostatitis symptoms}

How measured: NIH-CPSI score.

Time points measured: baseline, 3, 6 and 12 months.

Time points reported: baseline, 3, 6 and 12 months.

Subgroups: presence of benign prostate hyperplasia. 


\section{Urinary symptoms}

How measured: American Urologic Association Symptom Index.

Time points measured: baseline, and 3, 6 and 12 months.

Time points reported: baseline, and 3, 6 and 12 months.

Subgroups: presence of benign prostate hyperplasia.

\section{Adverse events}

How measured: narratively.

\begin{tabular}{ll}
\hline Funding sources & Not available. \\
\hline Declarations of interest & Not available. \\
\hline Notes & None.
\end{tabular}

\section{Risk of bias}

\begin{tabular}{|c|c|c|}
\hline Bias & Authors' judgement & Support for judgement \\
\hline $\begin{array}{l}\text { Random sequence genera- } \\
\text { tion (selection bias) }\end{array}$ & Low risk & $\begin{array}{l}\text { No information available in report. Contact with study author (Christof Kast- } \\
\text { ner) mentioned central allocation. }\end{array}$ \\
\hline $\begin{array}{l}\text { Allocation concealment } \\
\text { (selection bias) }\end{array}$ & Low risk & $\begin{array}{l}\text { No information available in report. Contact with study author (Christof Kast- } \\
\text { ner) mentioned central allocation. }\end{array}$ \\
\hline $\begin{array}{l}\text { Blinding of participants } \\
\text { and personnel (perfor- } \\
\text { mance bias) } \\
\text { Subjective outcomes }\end{array}$ & Unclear risk & $\begin{array}{l}\text { Participants blinded (information provided by Christof Kastner). For study per- } \\
\text { sonnel, unclear (blinding seemed unlikely). }\end{array}$ \\
\hline $\begin{array}{l}\text { Blinding of outcome as- } \\
\text { sessment (detection bias) } \\
\text { All outcomes }\end{array}$ & Low risk & Participants blinded (information provided by Christof Kastner). \\
\hline $\begin{array}{l}\text { Incomplete outcome data } \\
\text { (attrition bias) } \\
\text { All outcomes }\end{array}$ & High risk & $\begin{array}{l}42 \text { participants randomised, } 2 \text { participants excluded after randomisation due } \\
\text { to 'noncompliance with inclusion' and } 1 \text { participant excluded due to non-com- } \\
\text { pliance with a 'treatment criteria'. No information regarding treatment arms. } \\
\text { Additionally, } 4 \text { participants were lost at } 12 \text { months' follow-up (no available in- } \\
\text { formation regarding treatment arms). This would have likely affected outcome } \\
\text { data availability, but not specified for which outcomes. }\end{array}$ \\
\hline $\begin{array}{l}\text { Selective reporting (re- } \\
\text { porting bias) }\end{array}$ & Unclear risk & No protocol available. \\
\hline Other bias & Low risk & No other sources of bias identified. \\
\hline
\end{tabular}

Kessler 2014

$\begin{array}{ll}\text { Methods } & \text { Study design: parallel group randomised trial. } \\ \text { Study dates: November } 2009 \text { to July } 2012 . \\ \text { Setting: outpatient. }\end{array}$


Kessler 2014 (Continued)

\section{Country: Switzerland.}

\section{Participants}

Inclusion criteria: CPPS III, duration of symptoms $>3$ months, NIH-CPSI total score $>15$, NIH-CPSI pain score $>8$. Participants had been treated with a tetracycline for 4 weeks, alpha-blocker treatment for $\geq 6$ weeks and NSAID. All participants had written informed consent.

Exclusion criteria: inclusion criteria not fulfilled. Postvoid residual $>100 \mathrm{~mL}$, nitrite-positive urine sample, positive urine culture (Meares-Stamey 3-glass test and postprostatic massage urine). Urethral stricture, prostate cancer, aged $<18$ years, claustrophobia, cardiac pacemaker, implanted nerve-stimulator, insulin or pain pump.

Sample size: 60.

Age (years): Group 1: mean 49 (SD 14.2); Group 2: mean 44.9 (SD 15.9).

Baseline NIH-CPSI score: Group 1: 25.8; Group 2: 25.2.

Sex: men.

Interventions

Group 1 ( $n=30)$ : sono-electromagnetic therapy at home, using the portable Sonodyn device (Sonodyn Corporation AG, Solothurn, Switzerland), with gel applied to perineum twice daily and for 10 min (ultrasound intensity $100 \mathrm{~mW} / \mathrm{cm}^{2}$, ultrasonic power $12 \mathrm{~mW}$ and frequency $1.9 \mathrm{MHz}$, electric field force $0.3 \mathrm{~V} /$ $\mathrm{m}$ and magnetic field force of $0.4 \mathrm{~A} / \mathrm{m}$ ). Participants could not see settings and could not perceive the device. Duration of treatment 12 weeks.

Group 2 ( $n=30)$ : placebo device with the same characteristics: quote: 'They looked identical, were packed identically, and the placebo device lit the same buttons when charged and when switched on as the verum device, but did not provide stimulation'.

Cointerventions: not defined.

Outcomes Prostatitis symptoms

How measured: NIH-CPSI score.

Time points measured: baseline, and 6, 12 and 16 weeks.

Time points reported: baseline, and 6, 12 and 16 weeks.

Subgroups: age (cut-off point 50 years), symptom severity (cut-off point 25 points of NIH-CPSI score).

\section{Adverse events}

How measured: National Cancer Institute Common Terminology for Adverse Events version 4.

\section{Funding sources}

'This research was supported by the Sonodyn Corporation AG (Solothurn, Switzerland) and the University Hospital Inselspital (Bern, Switzerland). The funder had no role in study design, data collection and analysis, decision to publish, or preparation of the manuscript.'

Declarations of interest

'The authors declare that Sonodyn Corporation AG (Solothurn, Switzerland) provided all stimulation devices.'

Notes ClinicalTrials.gov NCT00688506.

\section{Risk of bias}

\begin{tabular}{lll}
\hline Bias & Authors' judgement & Support for judgement \\
\hline $\begin{array}{ll}\text { Random sequence genera- } \\
\text { tion (selection bias) }\end{array}$ & Low risk & $\begin{array}{l}\text { Quote: 'Computer-generated random numbers with a randomization ratio of } \\
1: 1 \text { and a block size of 60.' }\end{array}$ \\
\hline
\end{tabular}


Kessler 2014 (Continued)

$\begin{aligned} & \text { Allocation concealment } \\ & \text { (selection bias) }\end{aligned} \quad \begin{aligned} & \text { Quote: 'The manufacturer pre-packed and sequentially numbered the active } \\ & \text { and placebo devices according to the concealed randomization schedule.' }\end{aligned}$

Blinding of participants Low risk and personnel (perfor-

Quote: 'Patients, recruiting investigators, study nurses and physicians permance bias)

Subjective outcomes

\section{Blinding of outcome as- Low risk} sessment (detection bias) All outcomes forming follow-up assessments were all unaware of the allocated treatment.'

\begin{tabular}{lll}
\hline $\begin{array}{l}\text { Incomplete outcome data } \\
\text { (attrition bias) } \\
\text { All outcomes }\end{array}$ & Low risk & All outcomes: 'All patients completed 12 weeks of follow-up.' \\
\hline $\begin{array}{l}\text { Selective reporting (re- } \\
\text { porting bias) }\end{array}$ & Low risk & Outcome reporting matched protocol. \\
\hline Other bias & Low risk & No other sources of bias identified. \\
\hline
\end{tabular}

\section{Kucuk 2015}

\begin{tabular}{ll}
\hline Methods & Study design: parallel group randomised trial. \\
& Study dates: November 2008 to May 2009. \\
& Setting: academic hospital. \\
Country: Turkey.
\end{tabular}

Participants Inclusion criteria: aged 17-50 years with $\geq 12$ weeks of pelvic pain who underwent clinical and microbiological test (Meares-Stamey 4-glass test) and diagnosed as NIH category IIIb; all participants did not take any treatment before.

Exclusion criteria: participants with type IIla CP/CPPS, without sterile urine, suspicious digital rectal examination, elevated prostate-specific antigen, benign prostate hyperplasia, bleeding disorders, anticoagulated, localised skin infections, severe chronic diseases including cancer, autoinflammatory diseases, inflammatory bowel disease, irritable bowel syndrome or had urinary tract infection in last year.

\section{Sample size: 54.}

Age (years): overall 33.3 (SD 7.84).

Baseline NIH-CPSI score: Group 1: 22.92 (SD 7.36); Group 2: 20.36 (SD 7.35).

Sex: men.

Interventions $\quad$ Group $1(n=28)$ : levofloxacin $500 \mathrm{mg}$ daily and ibuprofen $200 \mathrm{mg}$ twice daily for 6 weeks.

Group 2 ( $n=26$ ): acupuncture group, UB 28 (bladder meridian), GB 41 (gallbladder meridian), LIV 3 (liver 3 meridian), LI 4 (large intestine 4 meridian), SP 6 (spleen 6 meridian), and SP 8 (spleen 8 meridian) acupuncture points used for stimulation using disposable acupuncture needles (Hua Long, $2540 \mathrm{~mm}$ Sterile Acupuncture Needles, China) and electrical pulse generator (Agistim Duo, $44 \mathrm{~mA} \mathrm{rms} \mathrm{max} / 99 \mathrm{~Hz}$ max, France), twice weekly for 7 weeks.

Cointerventions: not described.

Outcomes Prostatitis symptoms


How measured: NIH-CPSI.

Time points measured: baseline and 10 weeks.

Time points reported: baseline and 10 weeks.

\section{Adverse events}

How measured: narratively.

\begin{tabular}{|c|c|c|}
\hline Funding sources & \multicolumn{2}{|c|}{ No funding source provided. } \\
\hline Declarations of interest & \multicolumn{2}{|l|}{ Reported as 'none.' } \\
\hline Notes & \multicolumn{2}{|l|}{ None. } \\
\hline \multicolumn{3}{|l|}{ Risk of bias } \\
\hline Bias & Authors' judgement & Support for judgement \\
\hline $\begin{array}{l}\text { Random sequence genera- } \\
\text { tion (selection bias) }\end{array}$ & Unclear risk & $\begin{array}{l}\text { Quote: 'Patients were randomized into } 2 \text { groups prospectively.' No other infor- } \\
\text { mation provided. }\end{array}$ \\
\hline $\begin{array}{l}\text { Allocation concealment } \\
\text { (selection bias) }\end{array}$ & Unclear risk & $\begin{array}{l}\text { Quote: 'Patients were randomized into } 2 \text { groups prospectively.' No other infor- } \\
\text { mation provided. }\end{array}$ \\
\hline $\begin{array}{l}\text { Blinding of participants } \\
\text { and personnel (perfor- } \\
\text { mance bias) } \\
\text { Subjective outcomes }\end{array}$ & High risk & Open label study. \\
\hline $\begin{array}{l}\text { Blinding of outcome as- } \\
\text { sessment (detection bias) } \\
\text { All outcomes }\end{array}$ & High risk & Open label study. \\
\hline $\begin{array}{l}\text { Incomplete outcome data } \\
\text { (attrition bias) } \\
\text { All outcomes }\end{array}$ & Low risk & All outcomes: outcome data was available for all randomised participants. \\
\hline $\begin{array}{l}\text { Selective reporting (re- } \\
\text { porting bias) }\end{array}$ & Unclear risk & Protocol not available. \\
\hline Other bias & Low risk & No other sources of bias detected. \\
\hline
\end{tabular}

Lee 2008

Methods
Study design: parallel group randomised trial.
Study dates: February 2004 to July 2005.
Setting: academic hospitals, outpatient.
Country: Malaysia and US.

Participants Inclusion criteria: men aged $\geq 20$ years with NIH-CPSI total score $\geq 15$ (scale $0-43$ ) and symptoms for $\geq 3$ months within preceding 6 months. 
Lee 2008 (Continued)

Exclusion criteria: bacterial prostatitis, urinary tract infection within 1 year, any traditional or complementary alternative medicine (traditional Chinese medicine) treatment within 6 weeks or any consensus CP/CPPS exclusion criterion (Propert 2002).

Sample size: 89.

Age (years): Group 1: 40.9 (SD 11.0); Group 2: 42.8 (SD 9.4).

Baseline NIH-CPSI score: Group 1: 24.8 (SD 6.2); Group 2: 25.2 (SD 5.8).

Sex: men.

Interventions

Group $1(n=45)$ : 4 acupoints prepared, then sterile, disposable stainless steel needles (Suzhou HuanQiu Acupuncture Medical Supplies, Suzhou, China) placed perpendicularly in 30-min sessions in acupoints (CV1-Guan Yuan, CV4-Huiyin, SP6-Sanyinjiao and SP9-Yinlingquan), twice weekly for 10 weeks.

Group $2(n=45)$ : sham acupuncture included same number, duration and frequency of sessions as the acupuncture group at non-acupoints ( $15 \mathrm{~mm}$ to the left).

Cointerventions: no use of medications or supplemental therapies known to affect immune function during study.

Outcomes

\section{Prostatitis symptoms}

How measured: NIH-CPSI (continuous and dichotomous).

Dichotomous: responders defined as those who had a drop of 6 points in NIH-CPSI score.

Time points measured: baseline, and 5, 10, 14, 22 and 34 weeks.

Time points reported: baseline and 10 weeks; additionally, responder rate reported at 34 weeks. Other time points presented graphically.

\section{Urinary symptoms}

How measured: IPSS.

Time points measured: baseline, and 5, 10, 14, 22 and 34 weeks.

Time points reported: baseline and 10 weeks.

\section{Sexual dysfunction}

How measured: International Index of Erectile Function.

Time points measured: baseline, and 5, 10, 14, 22 and 34 weeks.

Time points reported: baseline and 10 weeks.

\section{Adverse events}

How measured: narratively.

\begin{tabular}{ll}
\hline Funding sources & NIH Grants DK065266 and DK38955, National Institutes of Health, Bethesda, MD. \\
\hline Declarations of interest & None. \\
\hline Notes & $\begin{array}{l}90 \text { participants randomised but } 1 \text { in acupuncture arm did not receive intervention (not analysed in re- } \\
\text { sults). } \\
\text { Clinical Trial registry: NCT00260637. }\end{array}$
\end{tabular}


Lee 2008 (Continued)

Bias Authors' judgement Support for judgement

Random sequence genera- Low risk Quote: 'computer generated, random, variable block design.'

tion (selection bias)

Allocation concealment Unclear risk No information provided.

(selection bias)

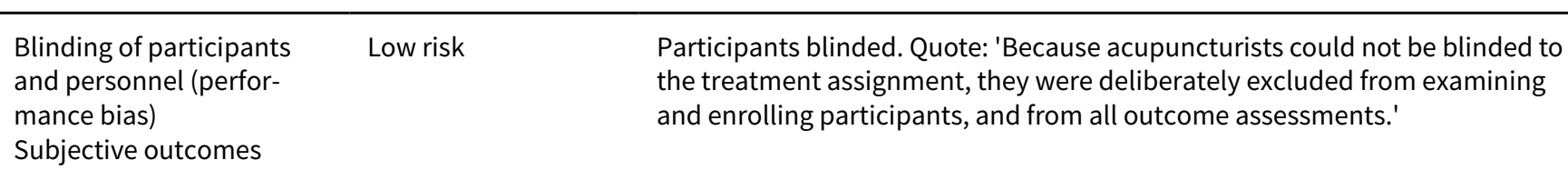

\begin{tabular}{|c|c|c|}
\hline $\begin{array}{l}\text { Blinding of outcome as- } \\
\text { sessment (detection bias) } \\
\text { All outcomes }\end{array}$ & Low risk & $\begin{array}{l}\text { Quote: 'Sham points were } 15 \mathrm{~mm} \text { away from the acupuncture points. These } \\
\text { measures ensured credibility, because three quarters of the sham group } \\
\text { thought they had received acupuncture.' }\end{array}$ \\
\hline
\end{tabular}

\begin{tabular}{|c|c|c|}
\hline $\begin{array}{l}\text { Incomplete outcome data } \\
\text { (attrition bias) } \\
\text { All outcomes }\end{array}$ & Low risk & $\begin{array}{l}\text { All outcomes: outcome data available for } 44 / 45 \text { participants allocated to } \\
\text { acupuncture and } 45 / 45 \text { participants allocated to sham procedure. }\end{array}$ \\
\hline $\begin{array}{l}\text { Selective reporting (re- } \\
\text { porting bias) }\end{array}$ & Low risk & All outcomes reported. \\
\hline Other bias & Low risk & No other sources of bias identified. \\
\hline
\end{tabular}

Lee 2009

Methods
Study design: parallel group randomised trial.
Study dates: June to December 2007.
Setting: academic hospital.
Country: Korea.

Participants Inclusion criteria: aged $>18$ years, NIH-CPSI total score $>15$ and symptoms for over 3 months during previous 6 months.

Exclusion criteria: urological disease (e.g. acute prostatitis or bacterial prostatitis, benign prostatic hyperplasia, prostate cancer, urinary tuberculosis, urinary tract infection, bladder stone, urethral stricture, interstitial cystitis, urethritis, neuropathic bladder, bladder cancer, haematuria), medication history (e.g. antibiotics, muscle relaxants, NSAIDs or analgesics within 1 month or presumed to have), diseases that influenced urological symptoms (e.g. brain disease, sexually transmitted disease), or any acute disease or disease requiring treatment. Participants aged $>50$ years excluded from study to minimise confounding role of benign prostatic hyperplasia-related symptoms and participants with a history of acupuncture within the previous 6 months.

Sample size: 39.

Age (years): Group 1: 39.8 (SD 5.8); Group 2: 36.4 (SD 5.8); Group 3: 38.2 (SD 6.9).

Baseline NIH-CPSI score: Group 1: 26.9 (SD 5.2); Group 2: 25.5 (SD 3.6); Group 3: 28 (SD 6.7).

Sex: men.

Interventions

Group 1 ( $n=12)$ : advice and exercise and $12 \times 20$-min sessions of electroacupuncture in 6 weeks. Advice consisted of hot sitz baths and 30 min of fast-paced walking on indoor-outdoor track. Electroacupunc- 
Lee 2009 (Continued)

ture therapy protocol included total of 6 acupuncture points at bilateral BL32 (zhongliao), BL33 (ciliao) and GB30 (huantiao) selected according to theory of neuroanatomy and myofascial pain syndromes; preparation alcohol pads and disposable stainless steel needles $(400.25 \mathrm{~mm}$, Dongbang Acupuncture, Chungnam, Republic of Korea); at GB30, disposable stainless steel needles (70 $0.30 \mathrm{~mm}$, Dongbang Acupuncture) inserted deeply to reach the myofascial trigger point of the piriformis muscle.

Group 2 ( $n=12$ ): advice and exercise and 12 sessions of sham electroacupuncture (included the same number and type of needle, duration and frequency of sessions as for the electroacupuncture treatment, but treatment delivered superficially at non-acupuncture points $15 \mathrm{~mm}$ to the lateral of each corresponding acupuncture point; points were not stimulated electrically, but sound of pulse generator was heard by participants).

Group $3(n=12)$ : advice and exercise alone.

Cointerventions: none.

Prostatitis symptoms
How measured: NIH-CPSI score.
Time points measured: baseline, and 3 and 6 weeks.
Time points reported: baseline, and 3 and 6 weeks.
Urinary symptoms
How measured: IPSS.
Time points measured: baseline, and 3 and 6 weeks.
Time points reported: baseline, and 3 and 6 weeks.

\section{Adverse events}

How measured: narratively.

\begin{tabular}{ll}
\hline Funding sources & Korea Science and Engineering Foundation grant funded by the Korean Government (MEST). \\
\hline Declarations of interest & Not available. \\
\hline Notes & Only active treatment and sham group included. \\
& Personal contact with author: Byung-Cheol Lee (hydrolee@korea.com). \\
\hline
\end{tabular}

\section{Risk of bias}

\begin{tabular}{|c|c|c|}
\hline Bias & Authors' judgement & Support for judgement \\
\hline $\begin{array}{l}\text { Random sequence genera- } \\
\text { tion (selection bias) }\end{array}$ & Low risk & Quote: 'Computer-generated, random block design.' \\
\hline $\begin{array}{l}\text { Allocation concealment } \\
\text { (selection bias) }\end{array}$ & Unclear risk & Not described. Wrote to study authors, information not available. \\
\hline $\begin{array}{l}\text { Blinding of participants } \\
\text { and personnel (perfor- } \\
\text { mance bias) } \\
\text { Subjective outcomes }\end{array}$ & Low risk & Personal contact with author: personnel and participants were blinded. \\
\hline $\begin{array}{l}\text { Blinding of outcome as- } \\
\text { sessment (detection bias) } \\
\text { All outcomes }\end{array}$ & Low risk & Personal contact with author: participants (outcome assessors) were blinded. \\
\hline
\end{tabular}


Lee 2009 (Continued)

\begin{tabular}{|c|c|c|}
\hline $\begin{array}{l}\text { Incomplete outcome data } \\
\text { (attrition bias) }\end{array}$ & Low risk & $\begin{array}{l}36 \text { participants randomised in } 3 \text { groups of } 12.1 \text { participant withdrew from } \\
\text { Group } 1 \text { and } 2 \text { from Group } 2 \text {. }\end{array}$ \\
\hline
\end{tabular}

All outcomes

Selective reporting (re- Unclear risk Wrote to study authors. No protocol available. porting bias)

Other bias Low risk No other sources of bias detected.

Leskinen 2002

Methods $\quad$ Study design: parallel group randomised trial (3:1 allocation ratio).

Study dates: February 1998 to May 2001.

Setting: national, multicentre.

Country: Finland.

Participants $\quad$ Inclusion criteria: moderate-to-severe symptoms of CPPS; > 6 months of CPPS symptoms.

Exclusion criteria: bacterial prostatitis, prostate cancer, excessive prostatic hyperplasia and any other abnormality that would affect study.

Sample size: 33 .

Age (years): Group 1: mean 42.8 (range 36-55); Group 2: mean 49.8 (range 45-53).

Baseline NIH-CPSI score: PSSI score: Group 1: mean 37.3 (SD 16.4); Group 2: mean 33.6 (SD 18.1).

Sex: men.

Interventions

Group 1 ( $n=25)$ : TUNA. 'The TUNA procedures were done using 465-kHz radiofrequency energy and the formal needle insertion technique as described by Issa 1996. Treatment was applied on two planes on both lateral lobes of the prostate in all patients so that the target temperature of $50^{\circ} \mathrm{C}$ at the needle tip was achieved for at least 1 minute.'

Group $2(n=8)$ : sham urethroscopy preformed so it was seemingly identical to TUNA intervention in participant's view.

Cointerventions: both interventions were done under spinal analgesia and light intravenous sedation. A single-dose of intravenous tobramycin $160 \mathrm{mg}$ used as antibiotic prophylaxis prior to interventions in all cases. Study participants were allowed to use ketoprofen as analgesic during follow-up with responsibility to keep record of drug intake.

\section{Prostatitis symptoms}

How measured: PSSI.

Time points measured: 3, 6 and 12 months.

Time points reported: baseline and 12 months (other time points presented graphically).

\section{Urinary symptoms}

How measured: IPSS.

Time points measured: 3,6 and 12 months.

Time points reported: baseline, and 3, 6 and 12 months. 
Leskinen 2002 (Continued)

\section{Adverse events}

How measured: narratively.

\begin{tabular}{ll}
\hline Funding sources & None. \\
\hline Declarations of interest & None. \\
\hline Notes & None. \\
\hline
\end{tabular}

\section{Risk of bias}

\begin{tabular}{|c|c|c|}
\hline Bias & Authors' judgement & Support for judgement \\
\hline $\begin{array}{l}\text { Random sequence genera- } \\
\text { tion (selection bias) }\end{array}$ & Unclear risk & $\begin{array}{l}\text { Quote: 'Patients... were randomized according to the closed-envelope method } \\
\text { at a 3:1 ratio to either TUNA treatment }(n=25) \text { or urethrocystoscopy as sham } \\
\text { treatment }(n=8) . \text { ' No additional information provided. }\end{array}$ \\
\hline $\begin{array}{l}\text { Allocation concealment } \\
\text { (selection bias) }\end{array}$ & Unclear risk & No information available. \\
\hline $\begin{array}{l}\text { Blinding of participants } \\
\text { and personnel (perfor- } \\
\text { mance bias) } \\
\text { Subjective outcomes }\end{array}$ & Unclear risk & $\begin{array}{l}\text { Quote: 'The patients were not told whether they received TUNA or sham treat- } \\
\text { ment, and to the patients' eyes, both procedures were designed to seem ex- } \\
\text { ternally alike. The TUNA equipment was visibly present in the operating room } \\
\text { during the sham procedures, and the cystoscope was left in the prostatic ure- } \\
\text { thra for } 20 \text { min to mimic the TUNA procedure as closely as possible.' }\end{array}$ \\
\hline & & $\begin{array}{l}\text { Considering the methodology, blinding of the participants was likely to have } \\
\text { been successful. But, since there was no information regarding blinding of oth- } \\
\text { er key study personnel, the judgement of risk of performance bias remained } \\
\text { unclear. }\end{array}$ \\
\hline
\end{tabular}

\begin{tabular}{lll}
\hline $\begin{array}{l}\text { Blinding of outcome as- } \\
\text { sessment (detection bias) } \\
\text { All outcomes }\end{array}$ & Unclear risk & No information provided. \\
\hline $\begin{array}{l}\text { Incomplete outcome data } \\
\text { (attrition bias) } \\
\text { All outcomes }\end{array}$ & Low risk & All outcomes: outcome data available for all randomised participants. \\
\hline $\begin{array}{l}\text { Selective reporting (re- } \\
\text { porting bias) }\end{array}$ & Unclear risk & Insufficient information for judgement. \\
\hline $\begin{array}{l}\text { Other bias } \\
\text { Unclear risk }\end{array}$ & $\begin{array}{l}\text { Baseline differences suggested that groups might not be comparable in terms } \\
\text { of duration of symptoms, but small sample size and 3:1 allocation ratio could } \\
\text { account for random differences. }\end{array}$ \\
\hline
\end{tabular}

Marx 2009

Methods Study design: parallel group randomised trial.

Study dates: 2003-2005.

Setting: academic hospital, outpatient.

Country: Germany.

Participants Inclusion criteria: aged 18-70 years, referred by urologists properly assessed with full examination.


Marx 2009 (Continued)

Exclusion criteria: chronic diseases (autoimmune, neoplastic, metabolic disease, benign hyperplasia, chronic cystitis), surgery in the lower abdomen.

Sample size: 35 .

Age (years): Group 1: 46.4 (SD 12.6); Group 2: 47.9 (SD 8.2).

Baseline NIH-CPSI score: Group 122.85 (SD 6.89); Group 2: 22.95 (SD 8.46).

Sex: men.

Interventions

Group 1 ( $n=20)$ : osteopathic treatment based of osteopathic theory of structural dysfunction; involved direct techniques (e.g. manipulation, mobilisation, muscle energy techniques, myofascial approaches) and indirect techniques (functional techniques, visceral techniques and cranial techniques). Prostate, coccygeal and rectal disorders were treated internally (rectal) and externally. Involved $5 \times 45$-minute sessions distributed in weeks $1,2,3,5$ and 8.

Group $2(n=15)$ : sham exercise programme with period of warm up, stretching, limb exercises, breathing exercises and pelvic floor exercise. Participants received $5 \times 30$-minute sessions.

Cointerventions: no other treatments allowed. Only medication prior to commencement of urogenital symptoms.

\section{Outcomes Prostatitis symptoms}

How measured: NIH-CPSI score and quality of life subscore.

Time points measured: baseline, 8 weeks (end of treatment), 6 weeks' follow-up after treatment and 1.5 years later (this time point only Group 1 ).

Time points reported: baseline, 8 weeks (end of treatment), 6 weeks' follow-up after treatment and 1.5 years later (this time point only Group 1).

\section{Urinary symptoms}

How measured: IPSS.

Time points measured: baseline, 8 weeks (end of treatment), 6 weeks' follow-up after treatment and 1.5 years later (this time point only Group 1 ).

Time points reported: baseline, 8 weeks (end of treatment), 6 weeks' follow-up after treatment and 1.5 years later (this time point only Group 1).

\begin{tabular}{lll}
\hline Funding sources & Not available. \\
\hline Declarations of interest & Authors declared no conflict of interests. \\
\hline Notes & There was a 5-year report of this trial but only of intervention arm. \\
\hline Risk of bias & Authors' judgement & Support for judgement \\
\hline Bias & Low risk & Computer-generated sequence. \\
\hline $\begin{array}{l}\text { Random sequence genera- } \\
\text { tion (selection bias) }\end{array}$ & Low risk & Central allocation. \\
\hline $\begin{array}{l}\text { Allocation concealment } \\
\text { (selection bias) }\end{array}$ & High risk & Participants and personnel not blinded. \\
\hline $\begin{array}{l}\text { Blinding of participants } \\
\text { and personnel (perfor- } \\
\text { mance bias) }\end{array}$ & &
\end{tabular}


Marx 2009 (Continued)

Subjective outcomes

Blinding of outcome as-
sessment (detection bias) $\quad$ High risk $\quad$ Participants not blinded.

sessment (detection bias)

All outcomes

Incomplete outcome data High risk

(attrition bias)

All outcomes: no outcome data for 2/15 participants in Group 2, whereas out-

All outcomes come data available for 20/20 participants in Group 1 (unbalanced attrition).

Selective reporting (re- Unclear risk $\quad$ Protocol not available.
porting bias)

porting bias)

Other bias Low risk No other sources of bias identified.

\section{Montorsi 1993}

$\begin{array}{ll}\text { Methods } & \text { Study design: parallel group randomised trial. } \\ \text { Study dates: November } 1987 \text { to July } 1991 . \\ \text { Setting: presumably national, outpatient. } \\ \text { Country: presumably Italy. }\end{array}$

Participants

Inclusion criteria: participants with chronic abacterial prostatitis or prostatodynia. Mean duration of subjective symptoms, as assessed by a modified Boyarsky scale, 2.3 years (range 8 months - 4 years). Of the participants with these diagnoses, only those who had experienced reoccurrence of the subjective symptoms after antibiotic therapy were eligible to enter study.

Exclusion criteria: type of pathology not explicitly stated. However, likely the authors excluded participants with bacterial prostatitis and prostate tumours if based on diagnostic tests to identify chronic abacterial participants.

Sample size: 54 .

Age (years): Mean age overall: 38.2 years (range 21 to 45); Group 1: 38.4; Group 2: 39.6; Group 3: 36.2.

Baseline NIH-CPSI score: not available.

Sex: men.

Group 2: 1 session of transrectal hyperthermia weekly for 6 weeks.

Group 3: 2 sessions of transrectal hyperthermia weekly for 3 weeks.

'The Prostathermer 99D system (Biodan Ltd, Rehovot, Israel) was used to deliver hyperthermia. The target temperature was reached within the first ten minutes of treatment and maintained throughout the whole session. Briefly, the system is composed of a rectal heat applicator with a source of microwaves at $915 \mathrm{MHz}$, and a series of thermosensors for monitoring rectal temperature, a cooling system for the anterior rectal wall, a specifically designed urethral catheter with three thermosensors for the assessment of prostatic urethra temperatures, and a computer system for data analysis and storage. Hyperthermia was administered on an outpatient basis and participants required only local anesthesia, with $2 \%$ xylocaine jelly before insertion of the catheter.'

Cointerventions: all the participants received antibiotic therapy with doxycycline $100 \mathrm{mg}$ twice daily for 2 weeks prior to trial. 
Montorsi 1993 (Continued)

Outcomes Adverse events

How measured: narratively.

\begin{tabular}{ll}
\hline Funding sources & None. \\
\hline Declarations of interest & None. \\
\hline Notes & Urinary symptoms measured using a modified Borsky scale (not a prespecified outcome of this review).
\end{tabular}

\section{Risk of bias}

\begin{tabular}{|c|c|c|}
\hline Bias & Authors' judgement & Support for judgement \\
\hline $\begin{array}{l}\text { Random sequence genera- } \\
\text { tion (selection bias) }\end{array}$ & Unclear risk & $\begin{array}{l}\text { Quote: 'Patients were randomly assigned to three different therapeutic proto- } \\
\text { cols, reported in Table 1.' No additional information provided. }\end{array}$ \\
\hline $\begin{array}{l}\text { Allocation concealment } \\
\text { (selection bias) }\end{array}$ & Unclear risk & No information available. \\
\hline $\begin{array}{l}\text { Blinding of participants } \\
\text { and personnel (perfor- } \\
\text { mance bias) } \\
\text { Subjective outcomes }\end{array}$ & High risk & Open label study. \\
\hline $\begin{array}{l}\text { Blinding of outcome as- } \\
\text { sessment (detection bias) } \\
\text { All outcomes }\end{array}$ & High risk & Open label study. \\
\hline $\begin{array}{l}\text { Incomplete outcome data } \\
\text { (attrition bias) } \\
\text { All outcomes }\end{array}$ & Low risk & All outcomes: outcome data available for all randomised participants. \\
\hline $\begin{array}{l}\text { Selective reporting (re- } \\
\text { porting bias) }\end{array}$ & Unclear risk & Insufficient information for judgement. \\
\hline Other bias & Unclear risk & $\begin{array}{l}\text { No information provided to compare the } 3 \text { groups on duration of symptoms. } \\
\text { Authors measured subjective symptoms using a tool of uncertain validity (Mc- } \\
\text { Naughton 2001). }\end{array}$ \\
\hline
\end{tabular}

Muraro 1995

\begin{tabular}{ll} 
Methods & Study design: parallel group randomised trial. \\
Study dates: not available. \\
Setting: outpatient. \\
Country: Italy. \\
\hline Participants \\
of symptoms. \\
Exclusion criteria: hypersensitivity to the drug, liver or kidney failure, neoplastic pathology of prostate \\
or positive cultures of urine or prostate secretions.
\end{tabular}


Muraro 1995 (Continued)

\section{Sample size: 20.}

Age (years): overall 42.5 (SD 6.8).

Baseline NIH-CPSI score: not available.

Sex: men.

Interventions

Group 1 ( $n=10)$ : Seaprose S (Flaminase, Formenti) $30 \mathrm{mg} 3$ times daily in combination with local hyperthermia, total of 7 sessions on alternate days of $60 \mathrm{~min}$ in duration, reaching local temperature of $42.5-43.5^{\circ} \mathrm{C}$.

Group $2(n=10): 7$ sessions of local hyperthermia alone.

Cointerventions: no anti-inflammatory treatment permitted.

\begin{tabular}{ll}
\hline Outcomes & None of the outcomes of the review were reported. \\
\hline Funding sources & Not available. \\
\hline Declarations of interest & Not available. \\
\hline Notes & $\begin{array}{l}\text { Seaprose } S \text { is a semi-alkaline crystallised protein derived from the purified culture of Aspergillus } \\
\text { melleus. }\end{array}$ \\
\hline
\end{tabular}

\section{Risk of bias}

\section{Bias}

Random sequence genera- Low risk tion (selection bias)

\section{Authors' judgement Support for judgement}

Randomisation performed using random numbers table (Fisher and Yates).

\begin{tabular}{lll}
\hline $\begin{array}{l}\text { Allocation concealment } \\
\text { (selection bias) }\end{array}$ & Unclear risk & No description of allocation concealment. \\
\hline $\begin{array}{l}\text { Blinding of participants } \\
\text { and personnel (perfor- } \\
\text { mance bias) } \\
\text { Subjective outcomes }\end{array}$ & High risk & Open label study. \\
\hline $\begin{array}{l}\text { Blinding of outcome as- } \\
\text { sessment (detection bias) } \\
\text { All outcomes }\end{array}$ & High risk & Open label study. \\
\hline $\begin{array}{l}\text { Incomplete outcome data } \\
\text { (attrition bias) } \\
\text { All outcomes }\end{array}$ & Unclear risk & No information available. \\
\hline $\begin{array}{l}\text { Selective reporting (re- } \\
\text { porting bias) }\end{array}$ & Unclear risk & No protocol available (none of the review outcomes were reported). \\
\hline \begin{tabular}{l} 
Other bias \\
\hline
\end{tabular} & Low risk & No other sources of bias identified. \\
\hline
\end{tabular}

Neimark 2016

$\begin{array}{ll}\text { Methods } & \text { Study design: parallel group randomised trial. } \\ \text { Study dates: study dates not available. }\end{array}$


Neimark 2016 (Continued)

Setting: outpatient, national.

Country: Russia.

\section{Outcomes Urinary symptoms}

How measured: IPSS.

Time points measured: baseline and 10 days (end of treatment).

Time points reported: baseline and 10 days (end of treatment).

\begin{tabular}{ll}
\hline Funding sources & None. \\
\hline Declarations of interest & None. \\
\hline Notes & $\begin{array}{l}\text { The active treatment apparatus invention and its patent belonged to the institution that conducted the } \\
\text { trial (Altai State Medical University). } \\
\text { Article in Russian. }\end{array}$ \\
\hline
\end{tabular}

\section{Risk of bias}

\begin{tabular}{|c|c|c|}
\hline Bias & Authors' judgement & Support for judgement \\
\hline $\begin{array}{l}\text { Random sequence genera- } \\
\text { tion (selection bias) }\end{array}$ & Unclear risk & No information available to make a judgement. \\
\hline $\begin{array}{l}\text { Allocation concealment } \\
\text { (selection bias) }\end{array}$ & Unclear risk & No information available to make a judgement. \\
\hline $\begin{array}{l}\text { Blinding of participants } \\
\text { and personnel (perfor- } \\
\text { mance bias) } \\
\text { Subjective outcomes }\end{array}$ & High risk & $\begin{array}{l}\text { No information available to make a judgement. Considering the visibly differ- } \\
\text { ent interventions, blinding was unlikely. }\end{array}$ \\
\hline $\begin{array}{l}\text { Blinding of outcome as- } \\
\text { sessment (detection bias) }\end{array}$ & High risk & $\begin{array}{l}\text { No information available to make a judgement. Considering the visibly differ- } \\
\text { ent interventions, blinding was unlikely. }\end{array}$ \\
\hline
\end{tabular}


Neimark 2016 (Continued)

All outcomes

Incomplete outcome data Low risk $\quad$ All outcomes: outcome data available for all participants.
(attrition bias)

(attrition bias)

All outcomes

Selective reporting (re- Unclear risk $\quad$ No protocol available.
porting bias)

porting bias)

Other bias Unclear risk Baseline characteristics poorly reported.

\section{Nickel 1996}

Methods Study design: randomised, double-blind, sham controlled trial.

Study dates: 'study dates not available.'

Setting: outpatient academic hospital.

Country: Canada.

Participants

Inclusion criteria: clinical diagnosis of non-bacterial prostatitis and with symptoms over 12 months; 10 WBC their prostate massage or post-prostatic massage urine. Participants had a symptom severity of $\geq 5$ ( 0 -10 scale for each domain) in 3 domains of the symptom severity index and $\geq 3$ ( 0 - 5 for each domain) in 3 domains of the symptom frequency questionnaire (total score $\geq 20$ ).

Exclusion criteria: positive cultures of expressed prostatic fluid or postprostatic massage urine, history of urinary tract infections, response to antibiotics.

Sample size: total 20.

Age (years): Group 1: mean 45.8; Group 2: mean 44.8.

Baseline NIH-CPSI score (PSSI): not available.

Sex: men.

Interventions

Group 1: transurethral microwave thermotherapy: single 1-hour treatment with a computer-driven device that elevated prostate interstitial temperatures to $45-60^{\circ} \mathrm{C}$, a range that does not cause significant necrosis of normal prostatic tissue.

Group 2: sham therapy: single 1-hour session with the same device using sham software.

Cointerventions: in phase 2 , all participants in both groups who did not show significant improvement after initial therapy were offered a 2 nd treatment; this phase was open labelled.

Outcomes

\section{Prostatitis symptoms}

How measured: symptom severity index and symptom frequency questionnaire.

Time points measured: 3 months (phase 1 ) and 21 months (phase 2).

Time points reported: 3 months and 21 months (in a subgroup).

\section{Urinary symptoms}

How measured: American Urology Association symptom score.

Time points measured: 3 months (phase 1 ) and 21 months (phase 2).

Time points reported: 3 and 21 months (in a subgroup). 
Nickel 1996 (Continued)

\section{Adverse events}

How measured: narratively.

\begin{tabular}{ll}
\hline Funding sources & Not available. \\
\hline Declarations of interest & Not available. \\
\hline Notes & None. \\
\hline
\end{tabular}

\section{Risk of bias}

\begin{tabular}{|c|c|c|}
\hline Bias & Authors' judgement & Support for judgement \\
\hline $\begin{array}{l}\text { Random sequence genera- } \\
\text { tion (selection bias) }\end{array}$ & Unclear risk & No information available. \\
\hline $\begin{array}{l}\text { Allocation concealment } \\
\text { (selection bias) }\end{array}$ & Unclear risk & No information available. \\
\hline $\begin{array}{l}\text { Blinding of participants } \\
\text { and personnel (perfor- } \\
\text { mance bias) } \\
\text { Subjective outcomes }\end{array}$ & Unclear risk & $\begin{array}{l}\text { Quote: 'The patient and evaluating urologist (J. C. N.) were blinded as to the } \\
\text { actual therapy performed. Phase } 1 \text { of the trial consisted of the randomized } \\
\text { double blind.' Unclear if all personnel were blinded. }\end{array}$ \\
\hline $\begin{array}{l}\text { Blinding of outcome as- } \\
\text { sessment (detection bias) } \\
\text { All outcomes }\end{array}$ & Low risk & $\begin{array}{l}\text { Quote: 'The patient and evaluating urologist (J. C. N.) were blinded as to the } \\
\text { actual therapy performed. Phase } 1 \text { of the trial consisted of the randomized } \\
\text { double blind.' }\end{array}$ \\
\hline $\begin{array}{l}\text { Incomplete outcome data } \\
\text { (attrition bias) } \\
\text { All outcomes }\end{array}$ & Low risk & All outcomes: outcome data available for all randomised participants. \\
\hline $\begin{array}{l}\text { Selective reporting (re- } \\
\text { porting bias) }\end{array}$ & Unclear risk & No protocol available. \\
\hline Other bias & Unclear risk & Baseline characteristics not available. \\
\hline
\end{tabular}

\section{Oh 2009}

\begin{tabular}{ll}
\hline Methods & Study design: parallel group randomised trial. \\
& Study dates: not available. \\
& Setting: not available. \\
Country: South Korea.
\end{tabular}

\begin{tabular}{ll}
\hline Participants & Inclusion criteria: not available. \\
& Exclusion criteria: not available. \\
& Sample size: 30. \\
& Age: not available. \\
& Sex: men.
\end{tabular}


Oh 2009 (Continued)

$\begin{array}{ll}\text { Interventions } & \text { Group } 1(\mathrm{n}=\text { not available): } \\ & \text { Group 2 }(\mathrm{n}=\text { not available): 'placebo therapy.' } \\ & \text { Cointerventions: not available. }\end{array}$

\section{Outcomes Prostatitis symptoms}

How measured: NIH-CPSI and Pelvic Pain Symptoms Survey.

Time points measured: baseline, and 1 month after treatment.

Time points reported: only $P$ value of 1 month after treatment.

\begin{tabular}{ll}
\hline Funding sources & None. \\
\hline Declarations of interest & Not available. \\
\hline Notes & $\begin{array}{l}\text { We extracted this information from an abstract presentation. We contacted the authors: Dr Oh } \\
\text { (mamah@hanmail.net) and Dr Bae (urobae@genetherapy.or.kr) and they mentioned that there was no } \\
\text { publication available. }\end{array}$
\end{tabular}

\section{Risk of bias}

\begin{tabular}{lll}
\hline Bias & Authors' judgement & Support for judgement \\
\hline $\begin{array}{l}\text { Random sequence genera- } \\
\text { tion (selection bias) }\end{array}$ & Unclear risk & Abstract only. \\
\hline $\begin{array}{l}\text { Allocation concealment } \\
\text { (selection bias) }\end{array}$ & Unclear risk & Abstract only. \\
\hline $\begin{array}{l}\text { Blinding of participants } \\
\text { and personnel (perfor- } \\
\text { mance bias) }\end{array}$ & Unclear risk & Abstract only. \\
$\begin{array}{l}\text { Subjective outcomes } \\
\text { Blinding of outcome as- } \\
\text { sessment (detection bias) }\end{array}$ & Unclear risk & Abstract only. \\
\hline $\begin{array}{l}\text { All outcomes } \\
\text { (attrition bias) } \\
\text { All outcomes }\end{array}$ & \\
\hline $\begin{array}{l}\text { Selective reporting (re- } \\
\text { porting bias) }\end{array}$ & Unclear risk & Abstract only. \\
\hline \begin{tabular}{l} 
Other bias \\
\hline
\end{tabular} & Unclear risk & Abstract only. \\
\hline
\end{tabular}

Paick 2006

Methods

Study design: parallel group randomised trial.

Study dates: December 2003 to July 2004.

Setting: outpatient, academic hospital. 
Paick 2006 (Continued)

\section{Country: South Korea.}

\section{Participants}

Inclusion criteria: aged $\geq 18$ years with pelvic pain defined as pain in bladder, groin, genitals or lower abdomen or perineal or (peri)anal areas (or a combination) without clear abnormalities on urological examination, and ability to communicate, understand and comply with requirements of study. These participants had type IIIb CP/CPPS.

Exclusion criteria: symptoms for $<6$ months, acute or chronic urethritis, urinary stones, bacterial or inflammatory CP/CPPS, bladder cancer, prostate cancer, urethral strictures, neurogenic bladder dysfunction, restricted mobility and antimicrobial or anti-inflammatory medication up to 4 weeks before enrolment in our study; documented history of prostatic intraepithelial neoplasia on biopsy, serum prostate-specific antigen levels $>20 \mathrm{ng} / \mathrm{mL}$, history of prostate surgery or radiotherapy and acute urinary retention or an indwelling catheter.

\section{Sample size: 40 .}

Age (years): Group 1: 49 (range 41.5-52); Group 2: 42 (28.8-49.5).

Baseline NIH-CPSI score: Group 1: 17 (IQR 13 to 24); Group 2: 21 (IQR 15.8 to 30).

Sex: men.

Interventions
Group 1 $(n=21)$ : terazosin (see cointerventions).
Group 2 $(n=19)$ : extracorporeal magnetic innervation using Neoconrol system (Neotonus Inc., Mari-
etta, GA, USA) that generated a magnetic field directed in seat of chair and concentrated in region of
pelvic muscles. 2 sessions weekly for 6 weeks, lasting 20 min each. The $1 \mathrm{st} 10 \mathrm{~min}$ used $10 \mathrm{~Hz}$ field, 2
min rest, and then an additional 10 min of $50 \mathrm{~Hz}$ field.
Cointerventions: all participants received terazosin $2 \mathrm{mg} /$ day for 1 st 7 days, and continued to receive 4
mg daily for following 5 weeks.

\section{Outcomes Prostatitis symptoms}

How measured: NIH-CPSI score.

Time points measured: before and after treatment ( 6 weeks).

Time points reported: before and after treatment ( 6 weeks).

\section{Urinary symptoms}

How measured: IPSS score.

Time points measured: before and after treatment ( 6 weeks).

Time points reported: before and after treatment ( 6 weeks).

\section{Adverse events}

How measured: narratively.

\begin{tabular}{ll} 
Funding sources & Not available. \\
\hline Declarations of interest & Not available. \\
\hline Notes & None.
\end{tabular}

\section{Risk of bias}


Paick 2006 (Continued)

Random sequence genera- Unclear risk tion (selection bias)
Quote: 'Patients were randomized according to the closed-envelop method at a 1:1 ratio to either terazosin monotherapy.

Unclear what method was used for random sequence generation.

Allocation concealment $\quad$ Unclear risk
(selection bias)

Allocation concealment Unclear risk (selection bias)
Quote: 'Patients were randomized according to the closed-envelop method at a 1:1 ratio to either terazosin monotherapy.'

Unclear whether they were opaque envelopes.

\begin{tabular}{|c|c|c|}
\hline $\begin{array}{l}\text { Blinding of participants } \\
\text { and personnel (perfor- } \\
\text { mance bias) } \\
\text { Subjective outcomes }\end{array}$ & High risk & Open label study. \\
\hline
\end{tabular}

\begin{tabular}{lll}
\hline Blinding of outcome as- & High risk & Open label study. \\
sessment (detection bias) &
\end{tabular}

sessment (detection bias)

All outcomes

$\begin{aligned} & \text { Incomplete outcome data } \\ & \text { (attrition bias) }\end{aligned}$ Low risk All outcomes: outcome data available for all randomised participants.
Alloutcomes

All outcomes

Selective reporting (re- Unclear risk $\quad$ Protocol not available.
porting bias)

\begin{tabular}{ll}
\hline Other bias $\quad$ Low risk $\quad$ No other sources of bias identified. \\
\hline
\end{tabular}

\section{Pajovic 2016}

\begin{tabular}{ll}
\hline Methods & Study design: parallel group randomised trial. \\
& Study dates: September 2013 to February 2015. \\
& Setting: outpatient. \\
Country: Montenegro.
\end{tabular}

\section{Participants}

Inclusion criteria: aged 30-50 years with category IIIb CP; score < 5 on items 1 and 2 (pain and discomfort) of NIH-CPSI; score $>4$ on item 9 (quality of life) of NIH-CPSI; participant had exhibited symptoms for $>3$ months and desired treatment.

Exclusion criteria: urinary tract infection documented within the last year; evidence of bacteria in seminal culture tests; met any NIH consensus exclusion criterion and had been treated or was taking medications that could affect lower urinary tract function; and prostate specific antigen level $>4 \mathrm{ng} / \mathrm{mL}$.

\section{Sample size: 60.}

Age (years): overall 39.4 (SD 4.4).

Baseline NIH-CPSI score: Group 1: 29.3 (SD 6.38); Group 2: 31.06 (SD 7.75).

Sex: men.

Interventions

Group 1 ( $n=30)$ : medical treatment with alpha-blocker (doxazosin 4 mg daily), anti-inflammatory agents (ibuprofen $400 \mathrm{mg}$ daily) and muscle relaxant (tiocolchicoside $12 \mathrm{mg}$ daily) in combination with 1 weekly 12-min ESWT session of 3000 impulses with total energy flow density $0.25 \mathrm{~mJ} / \mathrm{mm}^{2} 3 \mathrm{~Hz}$ for 12 weeks. 
Pajovic 2016 (Continued)

Group $2(n=30)$ : same medical treatment with alpha-blocker, anti-inflammatory agents and muscle relaxant, alone for 12 weeks.

\begin{tabular}{|c|c|c|}
\hline \multirow[t]{6}{*}{ Outcomes } & \multicolumn{2}{|l|}{ Prostatitis symptoms } \\
\hline & \multicolumn{2}{|c|}{ How measured: NIH-CPSI score. } \\
\hline & \multicolumn{2}{|c|}{ Time points measured: baseline, and 12 and 24 weeks. } \\
\hline & \multicolumn{2}{|c|}{ Time points reported: baseline, and 12 and 24 weeks. } \\
\hline & \multicolumn{2}{|l|}{ Adverse events } \\
\hline & \multicolumn{2}{|c|}{ How measured: number of events in each group. } \\
\hline Funding sources & \multicolumn{2}{|l|}{ None. } \\
\hline Declarations of interest & \multicolumn{2}{|l|}{ None. } \\
\hline Notes & \multicolumn{2}{|c|}{ Contact information: Marko Vukovic (marko.vukovic09@gmail.com) who replied our message. } \\
\hline \multicolumn{3}{|l|}{ Risk of bias } \\
\hline Bias & Authors' judgement & Support for judgement \\
\hline $\begin{array}{l}\text { Random sequence genera- } \\
\text { tion (selection bias) }\end{array}$ & Low risk & $\begin{array}{l}\text { No information available. Wrote to study authors: 'our randomisation process } \\
\text { was generated simply by computer system.' }\end{array}$ \\
\hline $\begin{array}{l}\text { Allocation concealment } \\
\text { (selection bias) }\end{array}$ & Low risk & $\begin{array}{l}\text { No information available. Wrote to study authors: 'allocation of patients in } \\
\text { treatment was 'envelope sealed.' }\end{array}$ \\
\hline $\begin{array}{l}\text { Blinding of participants } \\
\text { and personnel (perfor- } \\
\text { mance bias) } \\
\text { Subjective outcomes }\end{array}$ & High risk & Participants not blinded (subjective outcomes). \\
\hline $\begin{array}{l}\text { Blinding of outcome as- } \\
\text { sessment (detection bias) } \\
\text { All outcomes }\end{array}$ & High risk & Participants not blinded (subjective outcomes). \\
\hline $\begin{array}{l}\text { Incomplete outcome data } \\
\text { (attrition bias) } \\
\text { All outcomes }\end{array}$ & Low risk & $\begin{array}{l}\text { All outcomes: 'None of the patients were excluded from the study, and every- } \\
\text { body completed the study protocol during the follow-up period.' }\end{array}$ \\
\hline $\begin{array}{l}\text { Selective reporting (re- } \\
\text { porting bias) }\end{array}$ & Low risk & $\begin{array}{l}\text { Protocol not available. We wrote to study authors: 'Treatment protocol was } \\
\text { made according to previous studies made by Tugcu V et al } 2007 \text { and Zimmer- } \\
\text { mann R et al 2009.' Study by Zimmerman included in this review (Zimmermann } \\
\text { 2009). }\end{array}$ \\
\hline Other bias & Low risk & No other sources of bias identified. \\
\hline
\end{tabular}

\section{Rowe 2005}

Methods $\quad$ Study design: parallel group randomised trial.

Study dates: study dates not available.

Setting: outpatient. 
Rowe 2005 (Continued)

\section{Country: UK.}

Participants Inclusion criteria: aged $\leq 70$ years with diagnosis of CP/CPPS type IIIa or IIIb; each participant had previously undergone treatment with alpha-blockers, antibiotics and several other therapies.

Exclusion criteria: prostate cancer, pelvic radiotherapy, positive culture in prostatic secretion.

Sample size: 21.

Age (years): overall 47.8 (range 25-67).

Baseline NIH-CPSI score: PSSI: Group 1 mean: 38.8; Group 2 mean: 39.3.

Sex: men.

Interventions

Group 1 ( $n=11$ ): participants seated in Neotonus Electromagnetic Chair, for 2 consecutive 15-min periods (1st period $10 \mathrm{~Hz}, 2$ nd period $50 \mathrm{~Hz}$ ). Treatment included 2 sessions weekly for 4 weeks.

Group 2 ( $n=10)$ : participants seated in chair, ventilation mechanism activated, but no active stimulation applied.

Cointerventions: not available.

\section{Outcomes Prostatitis symptoms}

How measured: symptom questionnaire (9 items in a visual analogue scale, score 0 to 90); adapted from Nickel's questionnaire removing the digital rectal question (10 points). Pain and micturition subscores.

Time points measured: baseline, 3 months and 1 year.

Time points reported: baseline, 3 months and 1 year.

\section{Adverse events}

How measured: narratively.

\begin{tabular}{ll}
\hline Funding sources & 'Neotonus ${ }^{\mathrm{TM}}$ provided the electromagnetic chair.' \\
\hline Declarations of interest & Not available. \\
\hline Notes & None. \\
\hline
\end{tabular}

\section{Risk of bias}

\begin{tabular}{lll}
\hline Bias & Authors' judgement & Support for judgement \\
\hline $\begin{array}{l}\text { Random sequence genera- } \\
\text { tion (selection bias) }\end{array}$ & Low risk & Quote: 'computer generated, blocked randomization.' \\
\hline $\begin{array}{l}\text { Allocation concealment } \\
\text { (selection bias) }\end{array}$ & Unclear risk & No information available. \\
\hline $\begin{array}{l}\text { Blinding of participants } \\
\text { and personnel (perfor- } \\
\text { mance bias) }\end{array}$ & Unclear risk & $\begin{array}{l}\text { Participants blinded using a sham procedure; however, no information regard- } \\
\text { Subjective outcomes }\end{array}$ \\
\hline
\end{tabular}

\begin{tabular}{|c|c|c|}
\hline $\begin{array}{l}\text { Blinding of outcome as- } \\
\text { sessment (detection bias) }\end{array}$ & Low risk & $\begin{array}{l}\text { Quote: 'Patients were informed about the nature of the treatment, the treat- } \\
\text { ment schedule and the possibility that they might be randomized to placebo }\end{array}$ \\
\hline
\end{tabular}


Rowe 2005 (Continued)

but they were not given a detailed description of what local pelvic sensations, if any, to expect during treatment, so as not to bias blinding.'

Incomplete outcome data High risk
(attrition bias)

Follow-up data (all outcomes) available for all men. 11/11 in Group 1 and $7 / 10$

(attrition bias)

in Group 2 (3 did not complete treatment) at 3 months. 8/11 in Group 1 and

All outcomes

$5 / 10$ in Group 2 at 1 year. Data on micturition subscore not available for Group 1.

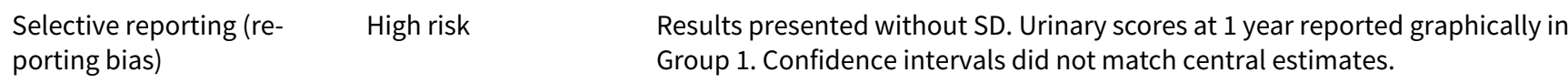

Other bias Low risk No other sources of bias detected.

Sahin 2015

Methods
Study design: parallel group randomised trial.
Study dates: study dates not available.
Setting: Department of Urology, Bakirkoy Dr Sadi Konuk Training and Research Hospital.
Country: Istanbul, Turkey.

Participants Inclusion criteria: lower urinary tract symptoms, and negative leucocyte and culture (ureaplasma, mycoplasma and chlamydia). Participants with CP/CPPS (NIH category IIIb) had history of disease refractory to standard conventional therapy including antibiotics, alpha-blockers and anti-inflammatory agents, and had symptoms of pain or discomfort in pelvic region for $\geq 3$ of previous 6 months.

Exclusion criteria: acute prostatitis or bacterial prostatitis, benign prostatic hyperplasia, prostate cancer, urinary tract infection within 1 year, pathology at urinary system ultrasonography including bladder and urethral stones, and any traditional or alternative medical therapy within past 6 weeks. Localised skin infections concerning the acupoints, bleeding diathesis and use of anticoagulation, as well as severe chronic or uncontrolled comorbid disease. Participants aged $>50$ years excluded to minimise confounding role of benign prostatic hyperplasia-related symptoms.

Sample size: 100 .

Age (years): 20-50.

Baseline NIH-CPSI score: Group 1: 27.0 (SD 3.5); Group 2: 26.5 (SD 3.7).

Sex: men.

Interventions

Group 1 ( $n=50)$ : acupuncture performed using 2 disposable stainless steel needles $(0.3 \mathrm{~mm}$ diameter, $60 \mathrm{~mm}$ length, Suzhou, Jiangsu, China) inserted to depth of maximum $2.5-3 \mathrm{~cm}$ in 7 acupoints bilaterally:

- BL-33 (Zhongliao): on sacrum, medial and inferior to posterosuperior iliac spine, just at 3rd posterior sacral foramen.

- BL-34 (Xialiao) on sacrum, medial and inferior to posterosuperior iliac spine, just at 4th posterior sacral foramen.

- BL-54 (Zhibian) on buttock and on level of 4 th posterior sacral foramen, 3 tsun ( $\sim 10 \mathrm{~cm}$ ) lateral to median sacral crest.

- CV-1 (Huiyin) on perineum, at midpoint between posterior border of scrotum and anus.

- CV-4 (Guanyuan) on lower abdomen and anterior midline, 3 tsun below centre of umbilicus.

- SP-6 (Sanyinjiao) 3 tsun above medial malleolus, posterior to medial border of tibia. 
Sahin 2015 (Continued)

- SP-9 (Yinlingquan) on medial surface of leg, just below medial condyle of tibia.

Overall treatment lasted $20 \mathrm{~min}$ in both groups and half of this period covered by needle stimulation through rotation, repeated weekly for 6 weeks without other treatment modalities.

Sham group ( $n=50)$ : punctures in sham group were performed $1 \mathrm{~cm}$ left of each selected acupoint, with same type of needles, of same duration and frequency.

Cointerventions: none.

Outcomes Prostatitis symptoms

How measured: NIH-CPSI score and subscores.

Time points measured: before and after treatment and on 6th, 8th, 16th and 24th week following treatment.

Time points reported: before and after treatment and on 6th, 8th, 16th and 24th week following treatment.

Subgroups: none.

\section{Adverse events}

How measured: narratively.

\begin{tabular}{ll}
\hline Funding sources & Not available. \\
\hline Declarations of interest & Authors declared no conflict of interest. \\
\hline Notes & None. \\
\hline
\end{tabular}

\section{Risk of bias}

\begin{tabular}{lll}
\hline Bias & Authors' judgement & Support for judgement \\
\hline $\begin{array}{l}\text { Random sequence genera- } \\
\text { tion (selection bias) }\end{array}$ & Low risk & Used computer-generated, random block design. \\
\hline
\end{tabular}

\begin{tabular}{|c|c|c|}
\hline $\begin{array}{l}\text { Allocation concealment } \\
\text { (selection bias) }\end{array}$ & Unclear risk & No information provided. \\
\hline $\begin{array}{l}\text { Blinding of participants } \\
\text { and personnel (perfor- } \\
\text { mance bias) } \\
\text { Subjective outcomes }\end{array}$ & Low risk & $\begin{array}{l}\text { Study blinded with a sham procedure. Acupuncturist not blinded, but they } \\
\text { were not involved in treatment of participants beyond procedure. }\end{array}$ \\
\hline $\begin{array}{l}\text { Blinding of outcome as- } \\
\text { sessment (detection bias) } \\
\text { All outcomes }\end{array}$ & Low risk & Participants blinded with a sham procedure. \\
\hline $\begin{array}{l}\text { Incomplete outcome data } \\
\text { (attrition bias) } \\
\text { All outcomes }\end{array}$ & Low risk & $\begin{array}{l}\text { Outcome data (all outcomes) available for } 45 / 50 \text { participants in Group } 1 \text { and } \\
46 / 50 \text { participants in Group } 2 \text {. Similar reasons for loss to follow-up reported. }\end{array}$ \\
\hline $\begin{array}{l}\text { Selective reporting (re- } \\
\text { porting bias) }\end{array}$ & Unclear risk & No protocol available. \\
\hline Other bias & Low risk & No other sources of bias identified. \\
\hline
\end{tabular}


Samhan 2011

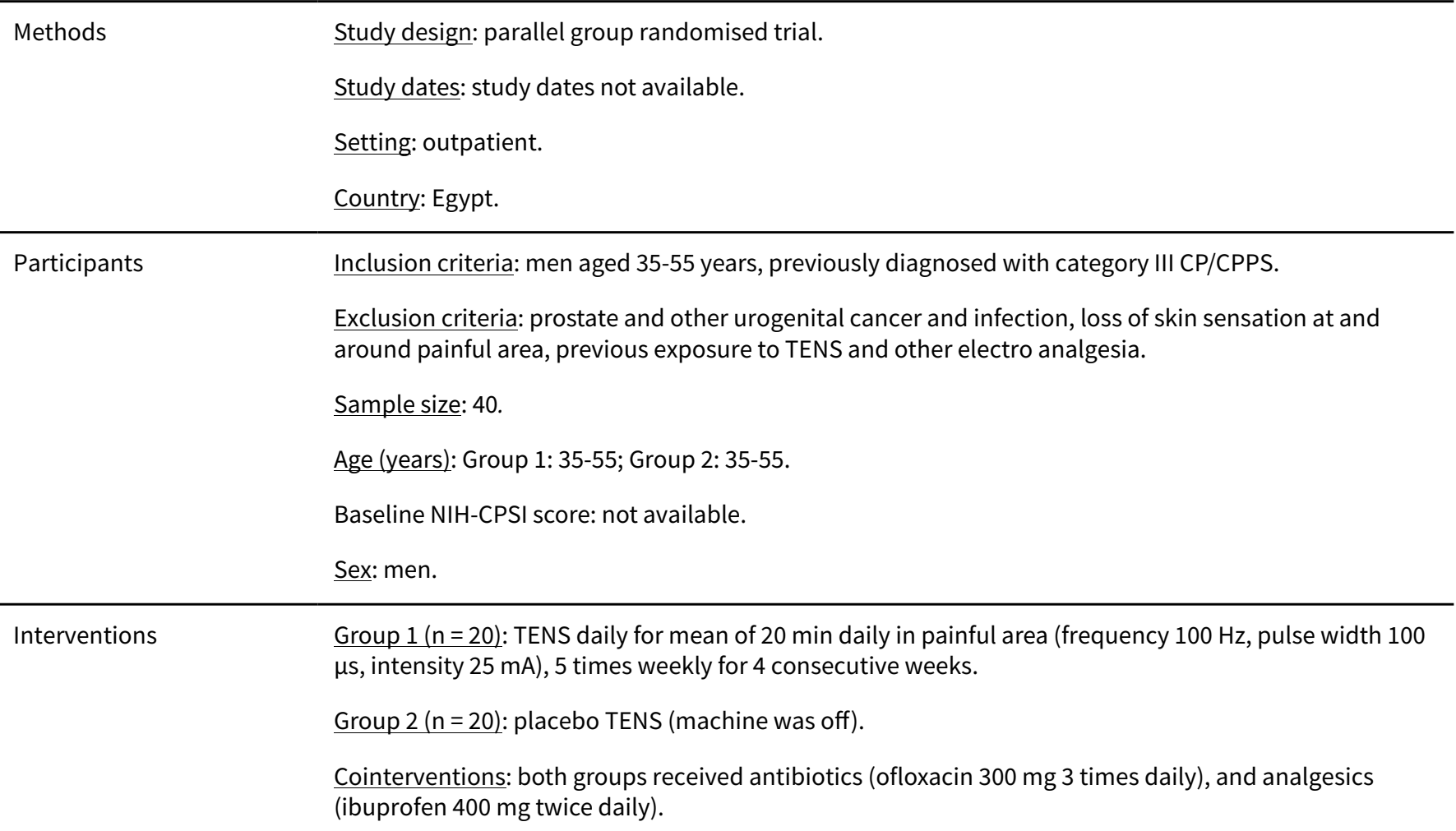

\begin{tabular}{ll}
\hline Outcomes & Prostatitis symptoms \\
& How measured: NIH-CPSI score: pain domain only. \\
& $\begin{array}{l}\text { Time points measured: } 0 \text { and } 4 \text { weeks. } \\
\text { Time points reported: } 0 \text { and 4 weeks. }\end{array}$ \\
\hline Funding sources & Not reported. \\
\hline Declarations of interest & Not reported. \\
\hline Notes & None. \\
\hline
\end{tabular}

\section{Risk of bias}

\begin{tabular}{lll}
\hline Bias & Authors' judgement & Support for judgement \\
\hline $\begin{array}{l}\text { Random sequence genera- } \\
\text { tion (selection bias) }\end{array}$ & Unclear risk & $\begin{array}{l}\text { Reported that sample was randomly recruited. However, no information on } \\
\text { method of randomisation. }\end{array}$ \\
\hline $\begin{array}{l}\text { Allocation concealment } \\
\text { (selection bias) }\end{array}$ & Unclear risk & $\begin{array}{l}\text { Indicated that allocating participants to the 2 groups was random. However, } \\
\text { method of allocation not reported. }\end{array}$ \\
\hline $\begin{array}{l}\text { Blinding of participants } \\
\text { and personnel (perfor- } \\
\text { mance bias) } \\
\begin{array}{l}\text { Subjective outcomes } \\
\hline\end{array}\end{array}$ & Unclear risk & $\begin{array}{l}\text { No information on blinding. Unlikely that blinding could have occurred since } \\
\text { TENS procedure switched off is substantially different from active treatment. }\end{array}$ \\
\hline
\end{tabular}


Samhan 2011 (Continued)

Blinding of outcome as- Unclear risk No information of blinding. Unlikely that blinding could have occurred since sessment (detection bias)

All outcomes

Incomplete outcome data Low risk Outcome data (all outcomes) available for all participants.

(attrition bias)

All outcomes

Selective reporting (re- Unclear risk No protocol available.

porting bias)

Other bias Low risk No other sources of bias identified.

Shah 1993

Methods
Study design: parallel group randomised trial.
Study dates: not available.
Setting: outpatient.
Country: UK.

\begin{tabular}{|c|c|c|}
\hline \multirow[t]{6}{*}{ Participants } & \multicolumn{2}{|c|}{ Inclusion criteria: chronic abacterial prostatitis and prostatodynia. } \\
\hline & \multicolumn{2}{|c|}{ Exclusion criteria: not available. } \\
\hline & \multicolumn{2}{|l|}{ Sample size: 30.} \\
\hline & \multicolumn{2}{|l|}{ Age: not available. } \\
\hline & \multicolumn{2}{|c|}{ NIH-CPSI score: not available. } \\
\hline & \multicolumn{2}{|l|}{$\underline{\text { Sex: }}$ men. } \\
\hline \multirow[t]{3}{*}{ Interventions } & \multicolumn{2}{|c|}{$\begin{array}{l}\text { Group } 1 \text { ( } n=15 \text { ): transrectal microwave therapy, } 4 \times 1 \text {-hour treatment sessions over } 2 \text { or } 3 \text { weeks. Tem- } \\
\text { perature raised to } 43.8^{\circ} \mathrm{C} \text { with input of } 40 \text { watts. }\end{array}$} \\
\hline & \multicolumn{2}{|c|}{ Group $2(n=15):$ 'Sham group.' Temperature $<37^{\circ} \mathrm{C}$. } \\
\hline & \multicolumn{2}{|c|}{ Cointerventions: not available. } \\
\hline Outcomes & \multicolumn{2}{|c|}{ None of the outcomes of the review were reported. } \\
\hline Funding sources & \multicolumn{2}{|l|}{ Not available. } \\
\hline Declarations of interest & \multicolumn{2}{|l|}{ Not available. } \\
\hline Notes & \multicolumn{2}{|c|}{ We extracted this information from an abstract presentation. No contact information available. } \\
\hline \multicolumn{3}{|l|}{ Risk of bias } \\
\hline Bias & Authors' judgement & Support for judgement \\
\hline $\begin{array}{l}\text { Random sequence genera- } \\
\text { tion (selection bias) }\end{array}$ & Unclear risk & Abstract only. \\
\hline
\end{tabular}


Shah 1993 (Continued)

\begin{tabular}{lll}
$\begin{array}{l}\text { Allocation concealment } \\
\text { (selection bias) }\end{array}$ & Unclear risk & Abstract only. \\
\hline $\begin{array}{l}\text { Blinding of participants } \\
\text { and personnel (perfor- } \\
\text { mance bias) }\end{array}$ & Unclear risk & Abstract only. \\
Subjective outcomes & &
\end{tabular}

$\begin{array}{lll}\text { Blinding of outcome as- } & \text { Unclear risk } & \text { Abstract only. } \\ \text { sessment (detection bias) } & \end{array}$

All outcomes

$\begin{aligned} & \text { Incomplete outcome data } \\ & \text { (attrition bias) }\end{aligned}$
Algh risk

All outcomes

Selective reporting (re- Unclear risk Abstract only.
porting bias)

\begin{tabular}{|c|c|c|}
\hline Other bias & Unclear risk & Abstract only. \\
\hline
\end{tabular}

\section{Shen 2006}

$\begin{array}{ll}\text { Methods } & \text { Study design: parallel group randomised trial. } \\ & \text { Study dates: June } 2002 \text { to December } 2005 . \\ & \text { Setting: outpatient setting. } \\ & \text { Country: China. }\end{array}$

\section{Participants}

Inclusion criteria: participants who meet the diagnostic criteria for chronic non-bacterial prostatitis.

Exclusion criteria: infection of urinary tract, EPS culture positive for bacteria, EPS routine test negative findings, benign prostatic hyperplasia, serious psychoneurosis, narrowing of urinary tract or prostate tumour. Participants with poor compliance and missing data were excluded.

Sample size: 72 .

Age (years): overall $20 \sim 46$, mean 31.

Baseline NIH-CPSI score: not available.

Sex: men.

Interventions $\quad$ Group $1(\mathrm{n}=40)$ : modified BiXieFenQing drink in morning and evening, $200 \mathrm{~mL}$ each + prostate massage once weekly.

Group 2 ( $n=32$ ): modified BiXieFenQing drink in morning and evening, $200 \mathrm{~mL}$ each.

Cointerventions: for participants with EPS mycoplasma culture positive, doxycycline given $0.1 \mathrm{~g}$ orally twice daily 2 weeks. Mycoplasma culture performed at weeks 1 and 2 after doxycycline treatment; if positive, another 2-week doxycycline treatment was given (abnormal liver function was a contradiction for this treatment).

\section{Outcomes Prostatitis symptoms}

How measured: NIH-CPSI subscores and 'clinical cure.' 
Shen 2006 (Continued)

Time points measured: before and after treatment.

Time points reported: before and after treatment.

\begin{tabular}{ll}
\hline Funding sources & Not mentioned. \\
\hline Declarations of interest & Not mentioned. \\
\hline Notes & $\begin{array}{l}\text { In the 72 participants included in this trial, 1 participant was complicated by epididymitis, } 7 \text { partici- } \\
\text { pants with mycoplasma culture positive. }\end{array}$ \\
\hline
\end{tabular}

\section{Risk of bias}

Bias Authors'judgement Support for judgement

Random sequence genera- Unclear risk Randomly assigned to Group 1 (40 participants) and Group 2 (32 participants) tion (selection bias) (in Chinese), but method for randomisation not mentioned.

\begin{tabular}{lll}
\hline $\begin{array}{l}\text { Allocation concealment } \\
\text { (selection bias) }\end{array}$ & Unclear risk & Allocation concealment not described. \\
\hline $\begin{array}{l}\text { Blinding of participants } \\
\text { and personnel (perfor- }\end{array}$ & High risk & $\begin{array}{l}\text { Blinding of participants and personnel not described. Considering the visibly } \\
\text { different interventions, blinding was unlikely. }\end{array}$
\end{tabular}
different interventions, blinding was unlikely.

mance bias)

Subjective outcomes

$\begin{array}{ll}\begin{array}{l}\text { Blinding of outcome as- } \\ \text { sessment (detection bias) }\end{array} & \text { High risk }\end{array} \quad \begin{aligned} & \text { Blinding of outcome assessment not described. Self-reported outcomes, see } \\ & \text { comment above. }\end{aligned}$
All outcomes

$\begin{array}{ll}\text { Incomplete outcome data Low risk } & \text { All outcomes: outcome data available for all participants. } \\ \text { (attrition bias) }\end{array}$

(attrition bias)

All outcomes

\begin{tabular}{lll}
\hline $\begin{array}{l}\text { Selective reporting (re- } \\
\text { porting bias) }\end{array}$ & Unclear risk & $\begin{array}{l}\text { Unclear whether there was selective outcome reporting (no protocol avail- } \\
\text { able). }\end{array}$ \\
\hline Other bias & Low risk & No other sources of bias identified. \\
\hline
\end{tabular}

Sikiru 2008

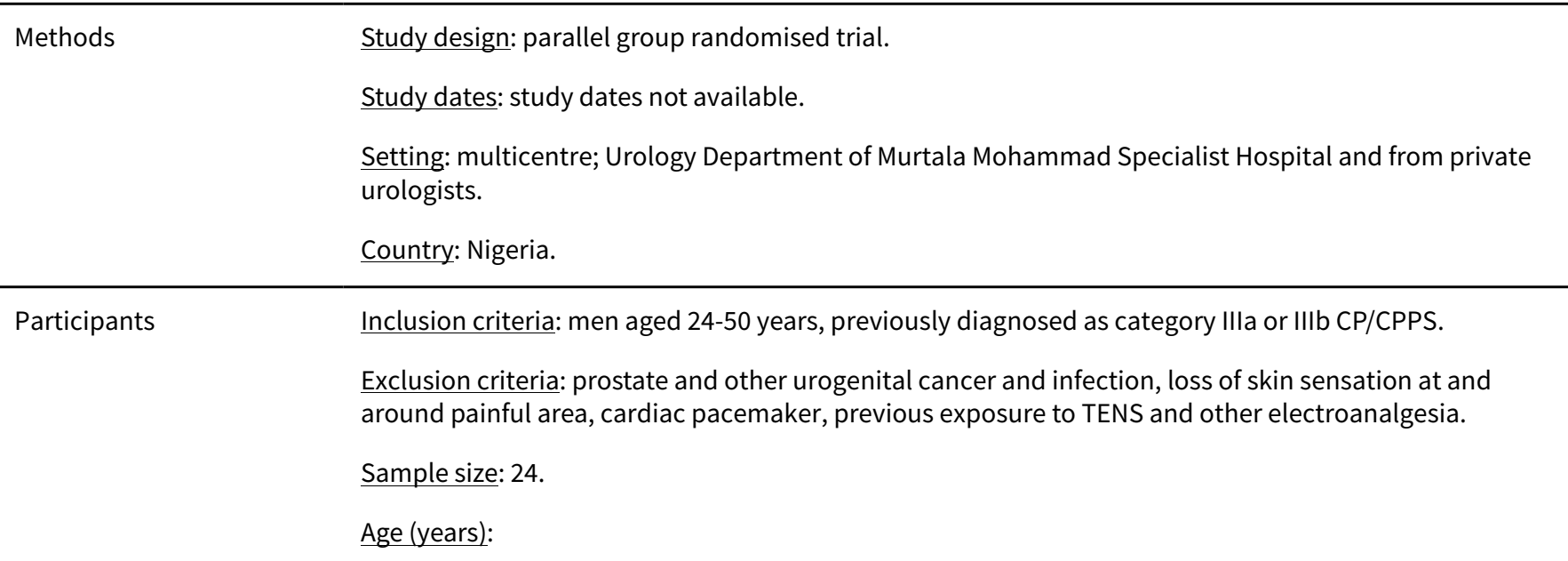


Sikiru 2008 (Continued)

Group TENS: range 24-50; mean 38.17 (SD 8.75).

Group Analgesic: range 23-55; mean 45.38 (SD 11.16).

Group Control: range 30-60; mean 46.83 (SD 8.16).

Baseline NIH-CPSI score: not available.

Sex: men.

Interventions

Group TENS ( $n=8$ ): antibiotics (ofloxacin $300 \mathrm{mg} 3$ times daily) + TENS. Participants stimulated with high TENS over painful area, daily for a mean of $20 \mathrm{~min}$, mean frequency $100 \mathrm{~Hz}$, pulse width $100 \mu \mathrm{s}$ and intensity $25 \mathrm{~mA}, 5$ times weekly for 4 consecutive weeks.

Group Analgesic ( $n=8)$ : ofloxacin 300 mg 3 times daily + ibuprofen 400 mg twice daily.

Group Control ( $n=8)$ : ofloxacin 300 mg 3 times daily + placebo tablets.

Cointerventions: none.

Outcomes Prostatitis symptoms

How measured: NIH-CPSI score: pain subscore.

Time points measured: 0 and 4 weeks.

Time points reported: 0 and 4 weeks.

Subgroups: none.

\begin{tabular}{ll}
\hline Funding sources & Not available. \\
\hline Declarations of interest & None declared. \\
\hline Notes & None. \\
\hline
\end{tabular}

\section{Risk of bias}

\begin{tabular}{|c|c|c|}
\hline Bias & Authors' judgement & Support for judgement \\
\hline $\begin{array}{l}\text { Random sequence genera- } \\
\text { tion (selection bias) }\end{array}$ & Unclear risk & $\begin{array}{l}\text { Reported that sample was randomly recruited. However, did not explain } \\
\text { method of randomisation. }\end{array}$ \\
\hline $\begin{array}{l}\text { Allocation concealment } \\
\text { (selection bias) }\end{array}$ & Unclear risk & $\begin{array}{l}\text { Indicated that allocating participants to the } 2 \text { groups was random. However, } \\
\text { no information on allocation method. }\end{array}$ \\
\hline $\begin{array}{l}\text { Blinding of participants } \\
\text { and personnel (perfor- } \\
\text { mance bias) } \\
\text { Subjective outcomes }\end{array}$ & High risk & $\begin{array}{l}\text { (Subjective outcomes.) Study investigators reported blinding of participants. } \\
\text { However, they reported that placebo was a tablet (visibly different from other } \\
\text { groups). }\end{array}$ \\
\hline $\begin{array}{l}\text { Blinding of outcome as- } \\
\text { sessment (detection bias) } \\
\text { All outcomes }\end{array}$ & High risk & $\begin{array}{l}\text { Study labelled as "blinded;" however, treatment group evaluating TENS was } \\
\text { visibly different from other groups. }\end{array}$ \\
\hline $\begin{array}{l}\text { Incomplete outcome data } \\
\text { (attrition bias) } \\
\text { All outcomes }\end{array}$ & Low risk & All outcomes: outcome data available for all participants. \\
\hline $\begin{array}{l}\text { Selective reporting (re- } \\
\text { porting bias) }\end{array}$ & Unclear risk & Protocol not available. \\
\hline
\end{tabular}


Sikiru 2008 (Continued)

Other bias High risk Control group had higher pain scores (mean 20.25) than the intervention group (mean 16.38).

Methods
Study design: parallel group randomised trial.
Study dates: October 2011 to October 2012.
Setting: Urology Clinic of Al-Zahra Hospital.
Country: Isfahan, Iran.

Participants Inclusion criteria: non-addiction to drugs and narcotics, chronic pelvic pain existence for $>3$ months, and certain diagnosis of chronic non-bacterial/CPPS defined as pain in bladder, groin, genitalia or lower abdomen, or perineal areas (or a combination) without clear abnormalities on urological examination.

Exclusion criteria: under treatment by another method at beginning of study, prostate cancer and bacterial prostatitis during workup, therapy plan alteration and non-inclination to continue project.

Sample size: 40.

Age (years):

Group 1: 35.4 (SD 8.4).

Group 2: 37 (SD 10.1).

Baseline NIH-CPSI score: Group 1: 26.5 (SD 3.4); Group 2: 27.1 (SD 3.1).

Sex: not applicable. Each time, 3000 impulses, with $0.25 \mathrm{~mJ} / \mathrm{mm}^{2}$ and $3 \mathrm{~Hz}$ of frequency delivered, although $0.5 \mathrm{~mJ} / \mathrm{mm}^{2}$ added in each week $\left(0.3 \mathrm{~mJ} / \mathrm{mm}^{2}\right.$ in week $2,0.35 \mathrm{~mJ} / \mathrm{mm}^{2}$ in week 3 , and $0.4 \mathrm{~mJ} / \mathrm{mm}^{2}$ in week 4). After each 500 pulses, probe position was corrected, using transperineal ultrasound.

Group $2(n=20)$ : sham group, same protocol applied but with probe being turned off.

Cointerventions: none.

\section{Outcomes Prostatitis symptoms}

How measured: NIH-CPSI score and subscores.

Time points measured: 1, 2, 3 and 12 weeks following the 1st ESWT session.

Time points reported: 1, 2, 3 and 12 weeks following the 1st ESWT session.

Subgroups: none.

\begin{tabular}{ll}
\hline Funding sources & Not reported. \\
\hline Declarations of interest & None known. \\
\hline Notes & ClinicalTrials.gov identifier: NCT01714830. \\
& $\begin{array}{l}\text { There were discrepancies in the outcome measures in } 2 \text { reports. We used the more recent report with } \\
\text { the more comprehensive follow-up. }\end{array}$
\end{tabular}


Vahdatpour 2013 (Continued)

Risk of bias

\begin{tabular}{|c|c|c|}
\hline Bias & Authors' judgement & Support for judgement \\
\hline $\begin{array}{l}\text { Random sequence genera- } \\
\text { tion (selection bias) }\end{array}$ & Unclear risk & $\begin{array}{l}\text { Reported that their sample was randomly recruited. No information on this } \\
\text { domain. We wrote to the authors for clarification. }\end{array}$ \\
\hline $\begin{array}{l}\text { Allocation concealment } \\
\text { (selection bias) }\end{array}$ & Unclear risk & No information on this domain. We wrote to the authors for clarification. \\
\hline $\begin{array}{l}\text { Blinding of participants } \\
\text { and personnel (perfor- } \\
\text { mance bias) } \\
\text { Subjective outcomes }\end{array}$ & Unclear risk & $\begin{array}{l}\text { Authors used the same device turned off as sham procedure; however, we } \\
\text { have no information on how effective was this method of masking, considering } \\
\text { the noticeably different procedures. We wrote to the authors for clarification. }\end{array}$ \\
\hline $\begin{array}{l}\text { Blinding of outcome as- } \\
\text { sessment (detection bias) } \\
\text { All outcomes }\end{array}$ & Unclear risk & $\begin{array}{l}\text { Authors used the same device turned off as sham procedure; however, we } \\
\text { have no information on how effective was this method of masking, considering } \\
\text { the noticeably different procedures. We wrote to the authors for clarification. }\end{array}$ \\
\hline $\begin{array}{l}\text { Incomplete outcome data } \\
\text { (attrition bias) } \\
\text { All outcomes }\end{array}$ & Unclear risk & $\begin{array}{l}\text { Differences in the } 2 \text { reports regarding number of participants. In the } 2013 \text { re- } \\
\text { port, they mentioned } 40 \text {, and in the } 2014 \text { report, they mentioned } 37 \text {. Unclear } \\
\text { if outcome measures (all outcomes) involved all participants. We wrote to the } \\
\text { authors for clarification. }\end{array}$ \\
\hline $\begin{array}{l}\text { Selective reporting (re- } \\
\text { porting bias) }\end{array}$ & Low risk & $\begin{array}{l}\text { No suspicion of selective reporting. Primary outcome defined in clinical trial } \\
\text { registry. }\end{array}$ \\
\hline Other bias & Low risk & No other sources of bias identified. \\
\hline
\end{tabular}

Vassily 1999

$\begin{array}{ll}\text { Methods } & \text { Study design: parallel group randomised trial. } \\ \text { Study dates: not available. } \\ \text { Setting: outpatient. } \\ \text { Country: Russia. } \\ \text { Inclusion criteria: chronic abacterial prostatitis. } \\ \text { Exclusion criteria: not available. } \\ \text { Sample size: } 120 . \\ \text { Age: not available. } \\ \text { Sex: men. } \\ \text { Group 1 } n=80 \text { ): transrectal microwave therapy, } 6 \text { sessions over } 2 \text { weeks each. } \\ \text { Group 2 ( } n=20): \text { 'sham group.' } \\ \text { Cointerventions: all participants received antibacterial agents. } \\ \text { Interventions } \\ \text { None of the outcomes of the review were reported. }\end{array}$


Vassily 1999 (Continued)

Funding sources Not available.

Declarations of interest Not available.

Notes We extracted this information from an abstract presentation. No contact information was available.

\section{Risk of bias}

\begin{tabular}{|c|c|c|}
\hline Bias & Authors' judgement & Support for judgement \\
\hline $\begin{array}{l}\text { Random sequence genera- } \\
\text { tion (selection bias) }\end{array}$ & Unclear risk & Abstract only. \\
\hline $\begin{array}{l}\text { Allocation concealment } \\
\text { (selection bias) }\end{array}$ & Unclear risk & Abstract only. \\
\hline $\begin{array}{l}\text { Blinding of participants } \\
\text { and personnel (perfor- } \\
\text { mance bias) } \\
\text { Subjective outcomes }\end{array}$ & Unclear risk & Abstract only. \\
\hline $\begin{array}{l}\text { Blinding of outcome as- } \\
\text { sessment (detection bias) } \\
\text { All outcomes }\end{array}$ & Unclear risk & Abstract only. \\
\hline $\begin{array}{l}\text { Incomplete outcome data } \\
\text { (attrition bias) } \\
\text { All outcomes }\end{array}$ & Unclear risk & Abstract only. \\
\hline $\begin{array}{l}\text { Selective reporting (re- } \\
\text { porting bias) }\end{array}$ & Unclear risk & Abstract only. \\
\hline Other bias & Unclear risk & Abstract only. \\
\hline
\end{tabular}

Wang 2002

Methods $\quad$ Study design: parallel group randomised trial.

Study dates: January 1998 to January 2001.

Setting: outpatient.

Country: China.

Participants

Inclusion criteria: not specified clearly. Participants who had a clinical diagnosis of CP.

Exclusion criteria: Meares-Stamey test and EPS routine test and culture used to exclude bacterial prostatitis. Urethral swab test used to exclude prostatitis caused by Neisseria gonorrhoeae, chlamydia or mycoplasma. Serum analysis for chlamydia and Herpes simplex virus used to exclude prostatitis caused by these micro-organisms.

Sample size: 136 (122 chronic nonbacterial prostatitis, 14 prostatodynia).

Age (years): overall: $25 \sim 54$, mean 34.2.

Baseline NIH-CPSI score: not available. 
Wang 2002 (Continued)

\section{Sex: men.}

Interventions

Group 1 ( $n=76$ ): external radiofrequency hyperthermia applied externally: 2 electrodes placed at hip and lower abdomen, $5^{\sim} 7 \mathrm{~cm}$ away from skin, with pubic symphysis as the centre $\left(42.5^{\sim} 43.5^{\circ} \mathrm{C}\right), 1^{\sim} 2$ hours each time, course of treatment: $2 \sim 3$ times, interval: $1 \sim 2$ weeks.

Additionally, terazosin taken orally $2 \mathrm{mg}$ every night for 2 days; if no serious dizziness or other adverse effects occurred, $2 \mathrm{mg}$ twice daily for 12 weeks after that.

Group 2 ( $n=90)$ : external radiofrequency hyperthermia: same as group 1.

No terazosin.

Cointerventions: none.

Prostatitis symptoms

How measured: symptom score questionnaire developed by Neal DE Jr and Moon TD (Neal 1994).

Time points measured: before and after treatment.

Time points reported: before and after treatment.

Subgroups: none.

\section{Adverse effects}

How measured: narratively.

\begin{tabular}{ll}
\hline Funding sources & Not mentioned. \\
\hline Declarations of interest & Not mentioned. \\
\hline Notes & None. \\
\hline
\end{tabular}

\section{Risk of bias}

\begin{tabular}{|c|c|c|}
\hline Bias & Authors' judgement & Support for judgement \\
\hline $\begin{array}{l}\text { Random sequence genera- } \\
\text { tion (selection bias) }\end{array}$ & Unclear risk & $\begin{array}{l}\text { ' } 136 \text { patients were randomly assigned to trial group ( } 76 \text { patients) and control } \\
\text { group ( } 60 \text { patients)' (in Chinese); however, method of randomisation not de- } \\
\text { scribed. }\end{array}$ \\
\hline $\begin{array}{l}\text { Allocation concealment } \\
\text { (selection bias) }\end{array}$ & Unclear risk & Allocation concealment not described. \\
\hline $\begin{array}{l}\text { Blinding of participants } \\
\text { and personnel (perfor- } \\
\text { mance bias) } \\
\text { Subjective outcomes }\end{array}$ & High risk & $\begin{array}{l}\text { Blinding of participants and personnel not described. Considering the visibly } \\
\text { different interventions, blinding was unlikely. }\end{array}$ \\
\hline $\begin{array}{l}\text { Blinding of outcome as- } \\
\text { sessment (detection bias) } \\
\text { All outcomes }\end{array}$ & High risk & $\begin{array}{l}\text { There were participant-reported outcomes, detection bias should be high (vis- } \\
\text { ibly different intervention). }\end{array}$ \\
\hline $\begin{array}{l}\text { Incomplete outcome data } \\
\text { (attrition bias) } \\
\text { All outcomes }\end{array}$ & Low risk & $\begin{array}{l}2 \text { participants in trial group had missing outcome data ( } 2.6 \% \text { of attrition) be- } \\
\text { cause of serious adverse effect (dizziness). }\end{array}$ \\
\hline $\begin{array}{l}\text { Selective reporting (re- } \\
\text { porting bias) }\end{array}$ & Unclear risk & $\begin{array}{l}\text { Unclear whether there was selective outcome reporting (no protocol avail- } \\
\text { able). }\end{array}$ \\
\hline
\end{tabular}


Wang 2002 (Continued)
Other bias
Low risk
No other sources of bias identified.

Yang 2011

Methods
Study design: parallel group randomised trial.
Study dates: June 2007 to December 2007.
Setting: outpatient.
Country: China.

Participants

Inclusion criteria: aged $\geq 18$ years, with course of disease of $\geq 3$ months with an NIH-CPSI pain subscore and urinary symptom subscore $\geq 1$ who had received antibiotics, alpha-blockers and NSAIDs treatment and were found ineffective.

Exclusion criteria: history of urinary system infection in last 3 months, history of urinary or rectal tumour, neurological disease and narrowing of urinary tract or history of urinary tract surgery.

Sample size: 140 .

Age (years): overall: $18^{\sim} 48$; mean: 30 .

Baseline NIH-CPSI score: Group A: 25.82 (SD 2.34); Group B: 26.92 (SD 3.18); Group C: 26.35 (SD 2.19); Group D: 25.3 (SD 6.09).

Sex: men.

Group A ( $n=20)$ : usual care (see cointerventions).

Group B ( $n=40)$ : biofeedback.

Display the EMG of the pelvic floor muscle to participant. Instruct participant on changes of EMG during contraction and relaxation of anus. Ask participant to contract (10 20 seconds) and relax (10 $20 \mathrm{sec}-$ onds) the anus according to instructions on display.

Repeatedly for 20 min, 5 times each week; total 2 weeks.

Group C ( $n=40)$ : electrical stimulation.

Electrical stimulation by anal electrodes. Intensity: $6^{\sim} 23 \mathrm{~mA}$, stimulation $10^{\sim} 20$ seconds, relaxation $10^{\sim} 20$ seconds.

Repeat the cycle for 20 min; 5 times each week; total 2 weeks.

Group D ( $n=40)$ : biofeedback + electrical stimulation.

Anus contraction (10 20 seconds) -> relaxation (10 20 seconds) -> electrical stimulation (Intensity: $6^{\sim} 23 \mathrm{~mA}, 10^{\sim} 20$ seconds) -> relaxation (10 20 seconds).

Repeat the cycle for 20 min; 5 times each week; total 2 weeks.

Cointerventions: avoid alcohol and spicy food; avoid sitting for too long and holding in urine; avoid catching a cold; be physically active and do exercise; have sex regularly; warm sitz bath regularly; discontinue any antibiotics, alpha-blockers and other medications during the trial; persistence in pelvic floor muscle training.

Outcomes

\section{Prostatitis symptoms}

How measured: NIH-CPSI global and subscore.

Time points measured: before and 1 month after treatment. 
Yang 2011 (Continued)

Time points reported: before and 1 month after treatment.

Subgroups: none.

\section{Adverse events}

How measured: narratively.

\begin{tabular}{ll}
\hline Funding sources & Not mentioned. \\
\hline Declarations of interest & Not mentioned. \\
\hline Notes & None. \\
\hline
\end{tabular}

\section{Risk of bias}

\begin{tabular}{|c|c|c|}
\hline Bias & Authors' judgement & Support for judgement \\
\hline $\begin{array}{l}\text { Random sequence genera- } \\
\text { tion (selection bias) }\end{array}$ & Unclear risk & $\begin{array}{l}\text { Paper reported that 'patients were randomly assigned to ...' However, we do } \\
\text { not know what the method of randomisation was. }\end{array}$ \\
\hline $\begin{array}{l}\text { Allocation concealment } \\
\text { (selection bias) }\end{array}$ & Unclear risk & Allocation concealment not described. \\
\hline $\begin{array}{l}\text { Blinding of participants } \\
\text { and personnel (perfor- } \\
\text { mance bias) } \\
\text { Subjective outcomes }\end{array}$ & High risk & $\begin{array}{l}\text { Paper did not report blinding of participants or personnel. However, the visi- } \\
\text { ble difference between the interventions made blinding unlikely. }\end{array}$ \\
\hline $\begin{array}{l}\text { Blinding of outcome as- } \\
\text { sessment (detection bias) } \\
\text { All outcomes }\end{array}$ & High risk & $\begin{array}{l}\text { Blinding of outcome assessment not described. Self-reported outcomes, par- } \\
\text { ticipants not blinded. }\end{array}$ \\
\hline $\begin{array}{l}\text { Incomplete outcome data } \\
\text { (attrition bias) } \\
\text { All outcomes }\end{array}$ & Low risk & All outcomes: outcome data available for all participants. \\
\hline $\begin{array}{l}\text { Selective reporting (re- } \\
\text { porting bias) }\end{array}$ & Unclear risk & Unclear whether there was selective outcome reporting. \\
\hline Other bias & Low risk & No other sources of bias identified. \\
\hline
\end{tabular}

Yoo 2009

\begin{tabular}{|c|c|}
\hline \multirow[t]{4}{*}{ Methods } & Study design: parallel group randomised trial. \\
\hline & Study dates: January 2005 to December 2010. \\
\hline & Setting: academic hospital. \\
\hline & Country: South Korea. \\
\hline \multirow[t]{2}{*}{ Participants } & $\begin{array}{l}\text { Inclusion criteria: participants had symptoms for }>3 \text { months, and all fulfilled the NIH diagnostic criteria } \\
\text { for CP/CPPS. }\end{array}$ \\
\hline & $\begin{array}{l}\text { Exclusion criteria: history of urethritis, epididymitis, varicocoele, perianal and rectal disorders, any } \\
\text { neurological disease, presence of neurogenic bladder, urethral stricture and previous urological } \\
\text { surgery. }\end{array}$ \\
\hline
\end{tabular}


Yoo 2009 (Continued)

\author{
Sample size: 132. \\ Age (years): \\ Group 1: 31.5 years (SD 6.7). \\ Group 2: 35.1 years (SD 8.9). \\ Group 3: 38.1 years (SD 8.0).
}

Baseline NIH-CPSI score: Group 1: 26.27 (SD 5.45); Group 2: 24.59 (SD 6.51); Group 3: 23.94 (SD 5.92).

Sex: men.

Group 2 ( $n=44)$ : transrectal microwave thermotherapy alone for 12 weeks; using a Uro-DR Device (Somang Medical; Kangreung, Korea), at an intrarectal temperature of $43^{\circ} \mathrm{C}$ for $30 \mathrm{~min}$, at a medium heating rate.

Group 3 ( $n=44)$ : transrectal microwave thermotherapy in combination with the treatment in group 1 for 12 weeks.

Prostatitis symptoms
How measured: NIH-CPSI score.
Time points measured: baseline, and 4, 8 and 12 weeks.
Time points reported: baseline and 12 weeks.

\title{
Adverse events
}

How measured: narratively.

\begin{tabular}{ll}
\hline Funding sources & Not available. \\
\hline Declarations of interest & No potential conflict of interest relevant to this article reported. \\
\hline Notes & $\begin{array}{l}\text { These characteristics were completed with the collaboration of the author Dr Chung (chung- } \\
\text { hong@kku.ac.kr) who provided the manuscript accepted for publication and additional information } \\
\text { (see 'Risk of bias' table). }\end{array}$
\end{tabular}

\section{Risk of bias}

\begin{tabular}{lll}
\hline Bias & Authors' judgement & Support for judgement \\
\hline $\begin{array}{l}\text { Random sequence genera- } \\
\text { tion (selection bias) }\end{array}$ & Low risk & $\begin{array}{l}\text { Random sequence generated with an Excel spreadsheet (information provided } \\
\text { by author). }\end{array}$ \\
\hline $\begin{array}{l}\text { Allocation concealment } \\
\text { (selection bias) }\end{array}$ & High risk & Allocation not concealed (information provided by author). \\
\hline $\begin{array}{l}\text { Blinding of participants } \\
\begin{array}{l}\text { and personnel (perfor- } \\
\text { mance bias) } \\
\text { Subjective outcomes }\end{array}\end{array}$ & High risk & Open label study. \\
\hline $\begin{array}{l}\text { Blinding of outcome as- } \\
\text { sessment (detection bias) } \\
\begin{array}{l}\text { All outcomes } \\
\hline\end{array}\end{array}$ & High risk & Open label study. \\
\hline
\end{tabular}


Yoo 2009 (Continued)

Incomplete outcome data High risk Unbalanced attrition: missing outcome data (all outcomes) in 7/44 partici(attrition bias) pants in Group 1, 3/44 participants in Group 2, 9/44 participants in Group 3.

All outcomes

Selective reporting (re- Unclear risk No protocol available.

porting bias)

Other bias Low risk No other sources of bias identified.

Zeng 2012

Methods Study design: parallel group randomised trial.

Study dates: August 2009 to May 2011.

Setting: academic hospital.

Country: China.

Participants

Inclusion criteria: aged $>18$ years with type IIIb CP/CPPS refractory to other treatments, with pelvic pain or discomfort defined as pain in bladder, groin, genitals or lower abdomen or perineal or perianal areas (or a combination) without clear abnormalities on urological examination for minimum 3 months, NIH-CPSI total score $>15$ and pain domain score $>4$, and the ability to communicate, understand and comply with the requirements of study.

Exclusion criteria: chronic urethritis, urinary stones, bacterial or inflammatory CP/CPPS, seminal vesiculitis, bladder cancer, prostate cancer, urethral strictures, neurogenic bladder dysfunction, restricted mobility and antimicrobial or anti-inflammatory medication within the 4 weeks prior to enrolment in the study. Participants were also excluded from analysis if they had a documented history of prostatic intraepithelial neoplasia on biopsy, serum prostate-specific antigen levels in excess of $4 \mathrm{ng} / \mathrm{mL}$, history of prostate surgery or radiotherapy, acute urinary retention or an indwelling catheter.

Sample size: 80.

Age (years):

ESWT group: 48.7 (SD 12.1).

Sham group: 46.3 (SD 10.2).

Baseline NIH-CPSI score: Group 1: 30.5 (SD 4.7); Group 2: 29.3 (SD 4.1).

Sex: men.

Interventions

Group 1 ( $n=40$ ): participants received 20,000 ESWT (HB-ESWT-01, Haibin Medical Equipment Co. Ltd., China) impulses in 10 sessions over 2 weeks. Shock waves were applied directly to perineal area in which the pain was localised (from anus to scrotum).

Starting energy density $0.06 \mathrm{~mJ} / \mathrm{mm}^{2}$ and frequency $2 \mathrm{~Hz}$ used for all treatments. The energy density was gradually increased until it reached the maximum possible tolerable pain level reported by participant. This energy density was recorded during 1 st session and used in all subsequent sessions.

Group 2 ( $n=40$ ): sham ESWT, which was conducted by setting energy level to 0 (no shockwave energy transmission), under conditions identical to Group 1.

Cointerventions: all participants had received prior treatment that consisted of antibiotics, anti-inflammatories, plant extracts, alpha1-blocker, 5-alpha-reductase inhibitors, antimuscarinics, anxiolytics and neuromodulation agents. 2 weeks prior to study, participants halted all medications used to control 
Zeng 2012 (Continued)

their specific prostatic symptoms. Throughout study, participants received no drugs that could influence the results, such as antibiotics, anti-inflammatories, antidepressants or pain relievers.

\section{Outcomes}

\section{Prostatitis score}

How measured: NIH-CPSI score.

Time points measured: 1 week before treatment (baseline), 1 week after initial treatment (mid-point), 2 weeks after initial treatment (end point), 4 weeks after end point ( 4 weeks' follow-up) and 12 weeks after end point (12 weeks' follow-up).

Time points reported: 0 and 2 weeks (the other time points were only available graphically).

\section{Adverse events}

How measured: narratively.

\begin{tabular}{ll}
\hline Funding sources & Not reported. \\
\hline Declarations of interest & 'None.' \\
\hline Notes & None. \\
\hline
\end{tabular}

\section{Risk of bias}

\begin{tabular}{|c|c|c|}
\hline Bias & Authors' judgement & Support for judgement \\
\hline $\begin{array}{l}\text { Random sequence genera- } \\
\text { tion (selection bias) }\end{array}$ & Unclear risk & $\begin{array}{l}\text { Reported that their sample was randomly recruited. No information for this } \\
\text { domain. We wrote to study authors for clarification. }\end{array}$ \\
\hline $\begin{array}{l}\text { Allocation concealment } \\
\text { (selection bias) }\end{array}$ & Low risk & $\begin{array}{l}\text { Indicated that allocating participants to the } 2 \text { groups was random using the } \\
\text { closed envelop technique. }\end{array}$ \\
\hline $\begin{array}{l}\text { Blinding of participants } \\
\text { and personnel (perfor- } \\
\text { mance bias) } \\
\text { Subjective outcomes }\end{array}$ & High risk & Quote: 'Single blind.' Subjective outcome. \\
\hline $\begin{array}{l}\text { Blinding of outcome as- } \\
\text { sessment (detection bias) } \\
\text { All outcomes }\end{array}$ & Unclear risk & $\begin{array}{l}\text { Authors used the same device turned off as sham procedure; however, no in- } \\
\text { formation on how effective this method of masking was, considering the no- } \\
\text { ticeably different procedures. We wrote to study authors for clarification. }\end{array}$ \\
\hline $\begin{array}{l}\text { Incomplete outcome data } \\
\text { (attrition bias) } \\
\text { All outcomes }\end{array}$ & Low risk & $\begin{array}{l}\text { All outcomes: } 2 / 40 \text { participants in Group } 1 \text { and } 3 / 40 \text { participants in Group } 2 \\
\text { had missing outcome data. }\end{array}$ \\
\hline $\begin{array}{l}\text { Selective reporting (re- } \\
\text { porting bias) }\end{array}$ & High risk & $\begin{array}{l}\text { No protocol available. Data presented graphically for NIH-CPSI score. Post hoc } \\
\text { analysis of responders was presented numerically. We wrote to study authors } \\
\text { for data. }\end{array}$ \\
\hline Other bias & Low risk & No other sources of bias identified. \\
\hline
\end{tabular}

\section{Zhang 2011a}

\begin{tabular}{ll}
\hline Methods & Study design: parallel group randomised trial. \\
& Study dates: not available.
\end{tabular}


Zhang 2011a (Continued)

Setting: outpatient.

Country: China.

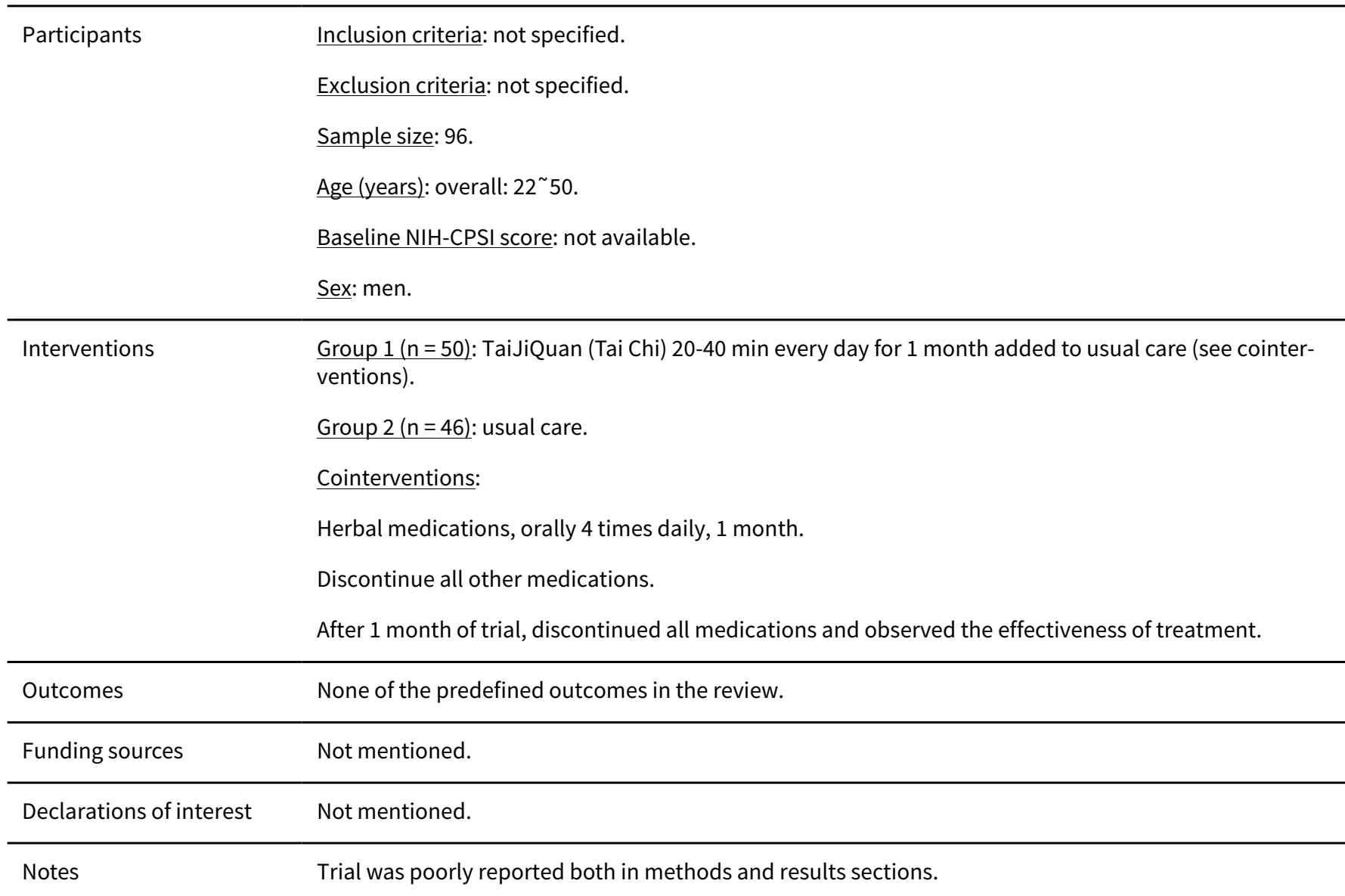

\section{Risk of bias}

Bias Authors' judgement Support for judgement

Random sequence genera- Unclear risk Quote: 'patients were randomly divided into control group and trial group' (in tion (selection bias) Chinese); however, unclear what exact method was.

\begin{tabular}{lll}
\hline $\begin{array}{l}\text { Allocation concealment } \\
\text { (selection bias) }\end{array}$ & Unclear risk & Allocation concealment not described. \\
\hline $\begin{array}{l}\text { Blinding of participants } \\
\text { and personnel (perfor- } \\
\text { mance bias) }\end{array}$ & High risk & Considering the visibly different interventions, blinding was unlikely. \\
Subjective outcomes & & \\
\end{tabular}

$\begin{array}{ll}\text { Blinding of outcome as- } & \text { High risk }\end{array}$ Considering the visibly different interventions, blinding was unlikely.

All outcomes

Incomplete outcome data Unclear risk No information available.
(attrition bias)

All outcomes 
Zhang 2011a (Continued)

Selective reporting (re- Unclear risk Unclear whether there was selective outcome reporting (no protocol availporting bias) able).

Other bias Low risk No other sources of bias identified.

Zhao 2015

\begin{tabular}{ll}
\hline Methods & Study design: parallel group randomised trial. \\
Study dates: June 2013 to March 2014. \\
Setting: hospital (surgery). \\
Country: China. \\
\hline Inclusion criteria: men aged 18-50 years with redundant prepuce and CP/CPPS with discomfort in the \\
pelvic region for $\geq 3$ months, urinary symptoms and sexual dysfunction, negative EPS bacterial culture, \\
pre- and postprostatic massage urine, and presence or absence of leukocytosis. \\
exclusion criteria: urinary tract infection; history of prostate cancer, inguinal hernia, inflammatory bow- \\
Sample size: 774. \\
Age (years): Group 1: 33 years (SD 10); Group 2: 33 years (SD 11). \\
Baseline NIH-CPSI score: Group 1: 21.0 (SD 7); Group 2: 21.0 (SD 8). \\
Sex: men.
\end{tabular}

Group $2(\mathrm{n}=390)$ : circumcision at 3 months (delayed).

Cointerventions: all participants received antibiotics (ciprofloxacin $500 \mathrm{mg}$ twice daily for 4 weeks), anti-inflammatory drugs (ibuprofen $400 \mathrm{mg}$ /day for 3 months), and alpha-blockers (tamsulosin $0.4 \mathrm{mg} /$ day for 3 months). All participants were told to refrain from sexual activity for 30 days and avoid spicy food, caffeine and alcohol.

Outcomes Prostatitis symptoms

How measured: NIH-CPSI score.

Time points measured: baseline and 12 weeks.

Time points reported: baseline and 12 weeks.

\section{Adverse events}

How measured: narratively.

\begin{tabular}{ll}
\hline Funding sources & Shanghai Public Health Bureau with additional support from Shanghai First People's Hospital. \\
\hline Declarations of interest & No competing interests. \\
\hline Notes & ChiCTRTRC14004567 (Clinical Trial Registry). \\
\hline
\end{tabular}

\section{Risk of bias}


Zhao 2015 (Continued)
Bias
Authors' judgement Support for judgement

Random sequence genera- Low risk

tion (selection bias)

Quote: 'were randomly assigned using computer-generated numbers into either the circumcision group or the control group.'

Allocation concealment Unclear risk No information available. Wrote to study authors.

(selection bias)

Blinding of participants High risk Open label study.
and personnel (perfor-
mance bias)
Subjective outcomes

\begin{tabular}{lll}
\hline Blinding of outcome as- & High risk & Open label study. \\
sessment (detection bias) &
\end{tabular}

All outcomes

\begin{tabular}{|c|c|c|}
\hline $\begin{array}{l}\text { Incomplete outcome data } \\
\text { (attrition bias) } \\
\text { All outcomes }\end{array}$ & Low risk & $\begin{array}{l}\text { Attrition low in both groups; 358/384 in Group } 1 \text { and 355/390 in Group } 2 \text { includ- } \\
\text { ed in analysis. }\end{array}$ \\
\hline
\end{tabular}

All outcomes

Selective reporting (re- Low risk $\quad$ Outcomes matched clinical trial registry.
porting bias)

\begin{tabular}{ll}
\hline Other bias $\quad$ Unclear risk & $\begin{array}{l}\text { Not reported how many of the participants received the treatment (circumci- } \\
\text { sion). Wrote to study authors. }\end{array}$
\end{tabular}

\section{Zimmermann 2009}

Methods
Study design: parallel group randomised trial.
Study dates: not mentioned.
Setting: Department of Urology, Elisabethinen Hospital.
Country: Linz, Austria.

Participants $\quad$ Inclusion criteria: participants with type IIIb prostatitis (CPPS) of at least 3 months' duration and no evi-
dence of bacteria in urinary and seminal culture tests.

Exclusion criteria: participants with other prostate pathologies, such as prostate cancer.

Sample size: 60 .

Age (years):

Treatment group: 42 (range: 22-52).

Placebo group: 43 (range: 34-61).

Baseline NIH-CPSI score: Group 1: 25.07 (SE 0.48); Group 2: 23.3 (SE 0.66).

Sex: not applicable.

Interventions

Group 1 ( $n=30)$ : 1 perineally applied ESWT treatment weekly (3000 pulses each; maximum total energy flow density: $0.25 \mathrm{~mJ} / \mathrm{mm}^{2}$; frequency: $3 \mathrm{~Hz}$ ) for 4 weeks. Position of shockwave transducer changed after every 500 pulses to scan virtually the entire prostatic and pelvic floor region. Device used for the study was standard electromagnetic shockwave unit with a focused shockwave source (Duolith SD1, Storz Medical, Tägerwilen, Switzerland). Focus zone penetration depth was 35-65 mm. 
Zimmermann 2009 (Continued)

Group $2(n=30)$ : placebo performed with same therapy head, which was also fitted with a placebo stand-off. This stand-off contained shock wave-absorbing material, a layer of air and air-filled microspheres.

Cointerventions: none.

Prostatitis symptoms
How measured: NIH-CPSI score and subscores.
Time points measured: 1,4 and 12 weeks following 1st session.
Time points reported: 1,4 and 12 weeks following 1 st session.
Subgroups: none.
Urinary symptoms
How measured: IPSS score.
Time points measured: 1,4 and 12 weeks following 1 st session.
Time points reported: 1,4 and 12 weeks following 1 st session.
Subgroups: none.
Sexual dysfunction
How measured: International Index of Erectile Function.
Time points measured: 1,4 and 12 weeks following 1 st session.
Time points reported: 1,4 and 12 weeks following 1 st session.
Subgroups: none.

\section{Adverse events}

How measured: narratively.

\begin{tabular}{ll}
\hline Funding sources & None. \\
\hline Declarations of interest & None known. \\
\hline Notes & None. \\
\hline
\end{tabular}

\section{Risk of bias}

\begin{tabular}{lll}
\hline Bias & Authors' judgement & Support for judgement \\
\hline $\begin{array}{l}\text { Random sequence genera- } \\
\text { tion (selection bias) }\end{array}$ & Unclear risk & $\begin{array}{l}\text { They reported that their sample was randomly recruited. However, did not ex- } \\
\text { plain their method of randomisation. }\end{array}$ \\
\hline $\begin{array}{l}\text { Allocation concealment } \\
\text { (selection bias) }\end{array}$ & Unclear risk & No information on selection bias. \\
\hline $\begin{array}{l}\text { Blinding of participants } \\
\begin{array}{l}\text { and personnel (perfor- } \\
\text { mance bias) }\end{array}\end{array}$ & Low risk & $\begin{array}{l}\text { Blinding included the specification that neither participant nor investiga- } \\
\text { tor/follow-up observer was aware of placebo or verum assignment. }\end{array}$ \\
\hline $\begin{array}{l}\text { Blinding of outcome as- } \\
\text { sessment (detection bias) }\end{array}$ & Low risk & $\begin{array}{l}\text { Blinding included the specification that neither participant nor investiga- } \\
\text { tor/follow-up observer was aware of placebo or verum assignment. }\end{array}$ \\
\hline
\end{tabular}


Zimmermann 2009 (Continued)

All outcomes

\begin{tabular}{lll}
$\begin{array}{l}\text { Incomplete outcome data } \\
\text { (attrition bias) } \\
\text { All outcomes }\end{array}$ & Low risk & All outcomes: outcome data available for all participants. \\
\hline $\begin{array}{l}\text { Selective reporting (re- } \\
\text { porting bias) }\end{array}$ & Low risk & $\begin{array}{l}\text { No suspicion of selective reporting. Reference of study protocol within study } \\
\text { report. }\end{array}$ \\
\hline Other bias & Low risk & No other sources of bias identified. \\
\hline
\end{tabular}

CFU: colony-forming unit; CP: chronic prostatitis; CPPS: chronic pelvic pain syndrome; EMG: electromyography; EPS: expressed prostate secretions; ESWT: extracorporeal shockwave therapy; IPSS: International Prostate Symptom Score; HPF: high power field; IQR: interquartile range; min: minute; n: number of participants; NIH: National Institutes of Health; NIH-CPSI: National Institutes of Health - Chronic Prostatitis Symptom Index; NSAID: non-steroidal anti-inflammatory drug; PSSI: Prostatitis Symptom Severity Index; PTNS: posterior tibial nerve stimulation; SD: standard deviation; SE: standard error; TENS: transcutaneous electrical nerve stimulation; TRFH: transrectal radiofrequency hyperthermia; TUNA: transurethral needle ablation; WBC: white blood cell.

Characteristics of excluded studies [ordered by study ID]

\begin{tabular}{|c|c|}
\hline Study & Reason for exclusion \\
\hline Aboumarzouk 2012 & Cochrane systematic review. \\
\hline Aliaev 2006 & Non-randomised comparative study for Sabal serrulata plant extract (study in Russian). \\
\hline Allen 2017 & Non-randomised controlled trial of thermobalancing therapy for CP/CPPS. \\
\hline Anothaisintawee 2011 & Systematic review. \\
\hline Barbalias 1998 & Included participants with bacterial prostatitis. CP/CPPS definition did not include pain. \\
\hline Bschleipfer 2007 & $\begin{array}{l}\text { "Intraprostatic botulinum toxin A injection" trial. Personal contact with author confirmed that tri- } \\
\text { al was stopped due to problems in recruiting (prospective participants with high levels of liver en- } \\
\text { zymes). }\end{array}$ \\
\hline Capodice 2005 & Systematic review. Complementary medicine. \\
\hline Chambo 2009 & Cochrane systematic review (Cochrane Review Group confirmed that this is a withdrawn protocol). \\
\hline Chang 2016 & Systematic review. Acupuncture. \\
\hline Chen 2006 & Systematic review. TCM. \\
\hline Chuang 2006 & Systematic review. Acupuncture. \\
\hline Cohen 2012 & Systematic review. \\
\hline Colleen 1975 & Non-randomised cross-over comparative study for minocycline. \\
\hline DRKS00009352 & Non-randomised controlled trial for physiotherapeutic device and thermobalancing therapy. \\
\hline Erickson 2008 & Systematic review. \\
\hline Evliyaoglu 2002 & Quasi-randomised study of doxazosin vs placebo. \\
\hline
\end{tabular}




\begin{tabular}{|c|c|}
\hline Study & Reason for exclusion \\
\hline Feng 2011 & Trial of nursing interventions included participants with bacterial prostatitis. \\
\hline Galeone 2012 & Prolexan trial. $60 \%$ had bacterial prostatitis, no disaggregated results available. \\
\hline Glybochko 2014 & Electrode plasmapheresis for chronic bacterial prostatitis. \\
\hline Golubchikov 2005 & Non-randomised controlled trial for a "combined treatment including complex physical factors." \\
\hline Hong 2008 & Abdominal cluster needle, quasi-randomised trial (randomisation based on date of admission). \\
\hline Ikeuchi 1990 & Study was assessed by Cochrane Japan collaborators. Non-randomised study for kampo medicine. \\
\hline ISRCTN43221600 & $\begin{array}{l}\text { Non-randomised study for a "combined psycho- and physiotherapeutic treatment program for pa- } \\
\text { tients with chronic pelvic pain syndrome (CPPS)." }\end{array}$ \\
\hline Jimenez-Pacheco 2014 & Abstract of a systematic review on "therapeutic alternatives." \\
\hline Kalinina 2015 & Non-randomised trial for a dietary supplement Prostatinol. \\
\hline Kamalov 2006 & Non-randomised trial for rectal suppositoria Vitaprost. \\
\hline Kogan 2010 & Non-randomised controlled trial for "magnetolaser therapy." \\
\hline Kotarinos 2009 & $\begin{array}{l}\text { Observational study secondary to a myofascial trigger point release RCT (included in twin re- } \\
\text { view "Non-pharmacological interventions for treating chronic prostatitis/chronic pelvic pain syn- } \\
\text { drome"). }\end{array}$ \\
\hline Le 2011 & Systematic review. \\
\hline Lee 2006 & Non-randomised study for "Uro-Vaxom." \\
\hline Lee 2007 & Systematic review. Alpha-blockers. \\
\hline Leng 2007 & Non-randomised study for a combination of TCM and Western medicine. \\
\hline Liu 2016 & Systematic review. Acupuncture for CP/CPPS. \\
\hline Lokshin 2010 & $\begin{array}{l}\text { Trial for "combination (ciprofloxacin+doxazosin) vs. monotherapy (ciprofloxacin)." Trial included } \\
\text { participants with bacterial prostatitis. }\end{array}$ \\
\hline Lopatkin 2009 & Non-randomised controlled study for vitaprost (translated from Russian). \\
\hline Loran 2003 & Non-randomised controlled study for Gentos. \\
\hline Ma 2015 & Quasi-randomised study for "catgut embedding therapy." \\
\hline Magistro 2016 & Systematic review. \\
\hline Marx 2013 & $\begin{array}{l}\text { Report of a single arm of the osteopathy trial after } 5 \text { years (included in the twin review "Non-phar- } \\
\text { macological interventions for treating chronic prostatitis/chronic pelvic pain syndrome"). }\end{array}$ \\
\hline McNaughton 2000 & Systematic review. \\
\hline McNaughton 2001 & Cochrane systematic review. \\
\hline
\end{tabular}




\begin{tabular}{ll}
\hline Study & Reason for exclusion \\
\hline McNaughton 2002 & Cochrane systematic review. Allopurinol. \\
\hline Mishra 2008 & Systematic review. \\
\hline NCT00194597 & Trial for Viagra (sildenafil). Study terminated due to illness of principal investigator. \\
\hline NCT00194623 & Study on Botox terminated since reorganisation of personnel forced termination. \\
\hline NCT00194636 & $\begin{array}{l}\text { Study on "Sympathetic Plexus Block" suspended participant recruitment (principal investigator } \\
\text { health issues). }\end{array}$ \\
\hline NCT00301405 & \begin{tabular}{l} 
Study on "Thalidomide" terminated. (Study closed. Difficult enrolment of men with prostatitis.) \\
\hline NCT00464373
\end{tabular} \\
$\begin{array}{l}\text { Trial of botulinum toxin type A as single intrasphincteric injection. Study terminated due to slow } \\
\text { accrual. }\end{array}$ \\
\hline NCT00529386 & $\begin{array}{l}\text { Personal contact with author (Dr Nickel): trial stopped for futility before endpoint, it was never } \\
\text { published, "8 patients received botox as per protocol } 1 \text { patient had mild improvement." Based on } \\
\text { slow enrolment and poor results, trial was discontinued. No follow-up or report other than to the } \\
\text { Institutional Review Board. }\end{array}$
\end{tabular}

\begin{tabular}{ll}
\hline NCT01678911 & Study on Gralise terminated due to difficulties in recruitment and low enrolment. \\
\hline NCT01830829 & $\begin{array}{l}\text { Study terminated due to difficulty in enrolling participants. Study for JALYN (dutasteride-tamsu- } \\
\text { losin combination). }\end{array}$ \\
\hline
\end{tabular}

\begin{tabular}{|c|c|}
\hline NCT02042651 & Study withdrawn prior to enrolment. \\
\hline Nickel 2011 & $\begin{array}{l}\text { Analysis of effects of dutasteride in men with CP/CPPS symptoms. Included participants not evalu- } \\
\text { ated to reach a diagnosis of CP/CPPS. RCT objective was prevention of prostate cancer. }\end{array}$ \\
\hline Osborn 1981 & Non-randomised study cross-over trial of muscle relaxants. \\
\hline Pavone 2010 & Non-randomised study of Serenoa repens for people with LUTS. \\
\hline Posadzki 2012 & Systematic review. Acupuncture. \\
\hline Pushkar' 2006 & $\begin{array}{l}\text { Active treatment and comparison group comprised of } 27 \%(6 / 22) \text { of people with bacterial prostati- } \\
\text { tis. No disaggregated data for people with CP/CPPS. }\end{array}$ \\
\hline Qin 2016a & Systematic review. Acupuncture. \\
\hline Qin 2016b & Systematic review. Medical therapy (network meta-analysis). \\
\hline Razumov 2005 & Non-randomised study of "combined physiotherapy." \\
\hline Simmons 1985 & $\begin{array}{l}\text { Definition of CP/CPPS did not match current definition. In fact, some participants had "non-specific } \\
\text { urethritis" and } 6 \text { participants were asymptomatic at beginning of study. }\end{array}$ \\
\hline Stamatiou 2014 & Quasi-randomised study of antibiotic vs phytotherapeutic therapy. \\
\hline Takahashi 2005 & $\begin{array}{l}\text { Study assessed by Cochrane Japan collaborators. Non-randomised study for levofloxacin and cer- } \\
\text { nitin pollen extract. }\end{array}$ \\
\hline Thakkinstian 2012 & Systematic review of "alpha-blockers, antibiotics and anti-inflammatories." \\
\hline
\end{tabular}




\begin{tabular}{|c|c|}
\hline Study & Reason for exclusion \\
\hline Thin 1983 & $\begin{array}{l}\text { Non-randomised study for the comparison of "minocycline, trimethoprim, co-trimoxazole or di- } \\
\text { azepam." }\end{array}$ \\
\hline Tkachuk 2006 & Non-randomised study for "Vitaprost." \\
\hline Tkachuk 2011 & Non-randomised study for "Vitaprost" as add-on therapy to physiotherapy. \\
\hline Wagenlehner 2017 & Phase II, dose-finding study with adaptive randomisation design. \\
\hline Xu 2004 & Non-randomised study of combination therapy (antibiotics, alpha-blockers, TCM, etc.). \\
\hline Yang 2006 & Systematic review. Alpha-blockers. \\
\hline Yang 2008 & Systematic review. \\
\hline Zhang 2011b & $\begin{array}{l}\text { Diagnosis of CP/CPPS was not according to the review definition. Authors referred to the presence } \\
\text { of both "Chinese medicine (CM) Gan (肝) - qi stagnancy syndrome type" and benign prostatic hy- } \\
\text { perplasia. }\end{array}$ \\
\hline Zhou 2017 & Definition of study population was not according to NIH criteria. \\
\hline
\end{tabular}

CP: chronic prostatitis; CPPS: chronic pelvic pain syndrome; LUTS: lower urinary tract symptom; NIH: National Institutes of Health; TCM: traditional Chinese medicine; RCT: randomised controlled trial.

Characteristics of studies awaiting assessment [ordered by study ID]

Rochester 2011

Methods $\quad$ 'Self Management Activation Randomised Trial for Prostatitis (SMART-P).'

Randomised controlled trial.

Participants

Inclusion criteria: men with CP/CPPS refractory to simple pharmacological manipulation from general urology clinics at Norfolk and Norwich University hospitals. Aged $>18$ years with CP/CPPS made by a urologist referred for 1 st time by their general practitioner. Participants must be refractory to antibiotic treatment.

Exclusion criteria: abnormal serum prostate specific antigen level; suspected prostate cancer on digital rectal examination; active urinary tract infection; alternative cause for pain found by urologist (e.g. ureteric calculus).

Age: adult.

Sex: men.

Target number of participants: 120.

Recruitment start date: 25 March 2011.

Recruitment end date: 24 March 2012. or pain clinic referral alone.

Standard care: after exclusion of a treatable bacterial cause for CP/CPPS, referral to pain clinic will be through agreement between clinician and participant. Pharmacological agents such as gabapentin, pregabalin and antidepressants are the mainstay of standard care at present. 
Intervention: this group will take part in a course of 6 weekly small group sessions (5-8 men) developed in conjunction with experts in psychology, pain management and urology, each lasting 1 hour with a focus on: understanding physiology of pain; psychological contributors; pain-coping mechanisms; behavioural responses; prevention, rehabilitation and re-enablement; relationships between symptom distress, emotion and pain.

Sessions will enable participants to learn techniques of problem solving and goal setting. Supported self-care and 'co-production' will be the underlying principles of this programme. The initial session is an introduction to the programme requirements, the rationale and the value of the approach. In early sessions, participants are instructed in the use of the Reaction Record for self-identifying and modifying catastrophic cognition and in understanding how such thinking is associated with greater negative affect, how there is little supportive evidence for such thinking, and how it can lead to poor choices in behavioural coping. During following sessions, participants identify and modify deficits in social support by practicing self-assertion communication exercises with their instructor and then later with significant others in their lives while using the Reaction Record to examine how to better negotiate distressing episodes. Further sessions use the Reaction Record tool to identify and modify illness-focused behavioural coping strategies and also to help re-engage the participant in physical and social activities that they may have abandoned. In the final session, participants are provided with a detailed review of their acquired behavioural modifications. Following this discussion, participants are instructed on continued problem-solving skills and future selfmanagement challenges are discussed.

\section{Primary outcomes:}

- changes in the NIH-CPPS, a validated scoring system for assessing the severity of the condition, which will be measured before and after the intervention.

\section{Secondary outcomes:}

- assess functional status (Hospital Anxiety and Depression and 36-item Short Form score);

- changes in requirements for pain-relief medication will also be used as a measure of effectiveness;

- participants degree of self-management/activation will be assessed by the Patient Activation Measure questionnaire;

- outcomes will be measured before and after the intervention.

Notes

Wrote to: kathryn.andrews@nnuh.nhs.uk (email returned).

Wrote to: mark.rochester@nnuh.nhs.uk (no response).

CP: chronic prostatitis; CPPS: chronic pelvic pain syndrome; NIH-CPPS: National Institute of Health Chronic Pelvic Pain Score.

Characteristics of ongoing studies [ordered by study ID]

ChicTR-IPR-16009262

Trial name or title
A Randomised Controlled Trial of Psychological Intervention Therapy in Patients with Category III Chronic Prostatitis/Chronic Pelvic Pain Syndrome.

Randomised parallel controlled trial.

Inclusion criteria: frequent urination, dribbling urine sense and perineum, testicles, lumbosacral pain, discomfort and other symptoms (history > 3 months); prostate massage fluid abnormalities; prostate massage fluid bacterial culture negative; no antibiotics and alpha-blocker previous treatment history.

Exclusion criteria: urinary tract infections, benign prostatic hyperplasia and other pelvic organs diseases.

Interventions Intervention: psychological intervention therapy. 
ChicTR-IPR-16009262 (Continued)

Control: routine treatment.

\begin{tabular}{ll}
\hline Outcomes & Primary outcome: \\
& - NIH-CPSI score. \\
& Secondary outcome: \\
& - IIEF score, SAS score, SDS score. \\
\hline Starting date & September 2016. \\
\hline Contact information & Wang Jianxin: wangzijx2009@aliyun.com. \\
\hline Notes & 'IPD will be public accessible via ResMan after 2019-03-01.' \\
\hline
\end{tabular}

NCT01828996

Trial name or title Shocking Therapy for Chronic Pelvic Pain Syndrome (CPPS).

Methods Randomised controlled trial, cross-over assignment.

Double blind (participants and care provider).

\begin{tabular}{|c|c|}
\hline \multirow[t]{2}{*}{ Participants } & $\begin{array}{l}\text { Inclusion criteria: exhibit symptoms of pain typical for prostatitis/CPPS; have had symptoms for } \geq \\
3 \text { months; have no evidence of infection in urine or expressed prostatic secretions (seminal plasm } \\
\text { may be substituted if expressed prostatic secretions not available); have failed } \geq 1 \text { therapy for CP- } \\
\text { PS. }\end{array}$ \\
\hline & $\begin{array}{l}\text { Exclusion criteria: suspected or confirmed to have prostate cancer; have a coagulation disorders; } \\
\text { use anticoagulants; have thrombosis; have used cortisone therapy up to } 6 \text { weeks before 1st treat- } \\
\text { ment; are actively trying to conceive. }\end{array}$ \\
\hline
\end{tabular}

Interventions Intervention: shockwave therapy, 4 sessions at 3000 pulses using Storz Duolith SD1 device.

Control: placebo procedure for 4 sessions.

Groups will cross-over after the 4 sessions.

\begin{tabular}{ll}
\hline Outcomes & Primary outcome: \\
& $\begin{array}{l}\text { Changes in pain at } 32 \text { weeks. Changes in pain is measured by a visual analogue scale from question } \\
4 \text { from the NIH-CPSI. }\end{array}$
\end{tabular}

\begin{tabular}{ll}
\hline Starting date & February 2013. \\
\hline Contact information & $\begin{array}{l}\text { Keith Jarvi, MD (Mount Sinai Hospital, Canada). No other contact information is provided in clinical } \\
\text { trial registry. }\end{array}$
\end{tabular}

Notes $\quad$ Estimated completion date: December 2017.

\section{NCT02588274}

\begin{tabular}{ll}
\hline Trial name or title & Efficacy of Acupuncture for Chronic Prostatitis/Chronic Pelvic Pain Syndromes. \\
\hline Methods & Randomised controlled trial, parallel group assignment. \\
\hline
\end{tabular}


NCT02588274 (Continued)

Double blind (participants and outcome assessors).

Participants

Age 18-50 years.

Inclusion criteria: history of pain perceived in region of prostate and absence of other lower urinary tract pathology for $\geq 3$ out of the past 6 months. In addition, associated lower urinary tract symptoms, sexual function and psychological factors should be addressed; age $18-50$ years; NIH-CPSI total score $\geq 15$ (scale $0-43$, and 0 means no symptom).

Exclusion criteria: other urological disease (e.g. acute prostatitis, bacterial prostatitis, benign prostatic hyperplasia, prostate cancer, urinary tuberculosis, urinary tract infection); serious or acute diseases with heart, liver, kidney and blood system; participants who had received acupuncture or medication (including alpha-blockers or pain killers) treatment in the week prior to the baseline assessment; participants without telephone number who cannot be connected during the follow-up.

Interventions

'Experimental: Acupuncture

Zhongliao (BL 33), Shenshu (BL 23), Huiyang (BL 35), and Sanyinjiao (SP 6) acupuncture points (Table 1). After patients are in prone position with relax, the investigators will use $75 \%$ alcohol pads to sterile the skin around the acupuncture points, and then insert steel needles (Huatuo, Suzhou, China $0.3 \mathrm{~mm}^{\star} 40 \mathrm{~mm} / 0.3 \mathrm{~mm}^{\star} 75 \mathrm{~mm}$ ) into the acupuncture points. For bilateral Zhongliao (BL 33), the needle will be inserted into about $50-60 \mathrm{~mm}$ with 45 degree, for Huiyang (BL 35), the needle will be inserted into 50-60 mm. for Shenshu (BL 23) and Sanyinjiao (SP 6), the needles will be inserted vertically to a depth of $25-30 \mathrm{~mm}$. The treatment sessions are 24 after baseline, 3 times a week, and the each time the patients will accept a 30 minutes treatment.'

'Placebo Comparator: placebo needle

The participants in placebo needle group will receive placebo needle at the same acupuncture points to treatment group.'

\begin{tabular}{ll}
\hline Starting date & November 2015. \\
\hline Contact information & Zhishun Liu: liuzhishun@aliyun.com. \\
\hline Notes & Estimated study end: May 2017. \\
\hline
\end{tabular}



tract pathology for $\geq 3$ of past 6 months. In addition, any associated lower urinary tract symptoms, sexual function and psychological factors should be addressed. Physical examinations, urine analyses, and urine cultures will be performed for all participants. Age 18-50 years. NIH-CPSI total score $\geq 15$.

Exclusion criteria: prostate, bladder or urethral cancer; seizure disorder in any medical history. Inflammatory bowel disease, active urethral stricture, neurological disease or disorder affecting the bladder, liver disease, neurological impairment or psychiatric disorder preventing understanding of consent and self-report scale. Urinary tract infection with a urine culture value $>100,000 \mathrm{CFU} /$ $\mathrm{mL}$, clinical evidence of urethritis, including urethral discharge or positive culture, diagnostic of sexually transmitted diseases (including gonorrhoea, chlamydia, mycoplasma or trichomonas, but not including HIV/AIDS), symptoms of acute or chronic epididymitis). Residual urine volume $\geq$ $100 \mathrm{~mL}$. $Q_{\max } \leq 15 \mathrm{~mL} /$ second. Prior 4 weeks used androgen hormone inhibitors (finasteride), alpha-blockers (terazosin hydrochloride, doxazosin mesylate, tamsulosin hydrochloride), antibiotics (ciprofloxacin hydrochloride) or any other prostatitis-specific medication (including herb and Chinese medicine).

Participants will receive treatment that consists of 20 acupuncture sessions over an 8-week ( 3 sessions in each of 1 st 4 weeks, and 2 sessions in each of remaining 4 weeks) period after baseline, each for $30 \mathrm{~min}$. Hwato brand disposable acupuncture needles (size $0.30 \times 75 \mathrm{~mm}$; size $0.30 \times 40$ $\mathrm{mm}$ ) will be used. Sanyinjiao (SP6), Zhongliao (BL33), Shenshu (BL23) and Huiyang (BL35) were selected as acupoints protocol. SP6 is on the tibial aspect of the leg, posterior to the medial border of the tibia, 3 cun superior to the prominence of the medial malleolus; BL23 is in the sacral region, in the 2nd posterior sacral foramen; BL33 is in the third posterior sacral foramen; BL35 is in the buttock region, 0.5 cun lateral to the extremity of the coccyx.

Sham comparator: sham acupuncture.

Participants will receive shallow needling at bilateral sham BL23, BL33, BL35 and SP6. Protocol includes same duration and frequency of sessions as for the acupuncture treatment, but treatment was delivered superficially at non-acupuncture points $10-15 \mathrm{~mm}$ to the lateral of corresponding acupuncture and not above a meridian line ( $15 \mathrm{~mm}$ to BL23, BL33 and BL35; $10 \mathrm{~mm}$ to SP6). The Hwato brand disposable acupuncture needles (size $0.30 \times 25 \mathrm{~mm}$ ) will be inserted with a depth of 2-3 mm without any manipulation.

\section{Outcomes} Primary outcomes:

- proportion of responders at end of 8 weeks: responder defined as who has a decline of $\geq 6$ points from baseline measured using NIH-CPSI;

- proportion of responders at end of 32-week.

Secondary outcomes:

- proportion of responders in 2 groups: week 1-7; week 24;

- change from baseline in NIH-CPSI total score and subscales (time frame: weeks 1-8, 24 and 32);

- change from baseline in IPSS (time frame: weeks 4, 8, 24 and 32);

- change from baseline in (HADS) (time frame: weeks 8, 24 and 32);

- change from baseline in IIEF-5 (time frame: weeks 8, 24 and 32);

- change from baseline in the EuroQol (EQ-5D) questionnaire (time frame: weeks 8, 24 and 32);

- response rate proportion of GRA (time frame: weeks 4, 8, 24 and 32);

- change for peak and mean urinary flow rate from baseline: week 8 .

Other outcome:

- expectation assessment (time frame: week 0 (baseline)): assessed at baseline, which includes 2 brief questions to investigate whether participants are confident that acupuncture treatment will 
NCT03213938 (Continued)

help their CP/CPPS: 'In general, is acupuncture effective for controlling the illness?', 'Do you think acupuncture will helpful to improve your CP/CPPS symptoms?' For each question, participants could choose 'yes,' 'no' or 'unclear' as the answer.

\begin{tabular}{ll}
\hline Starting date & 9 October 2017. \\
\hline Contact information & Zongshi Qin +861788880 8861; arisq@foxmail.com. \\
\hline Notes & Estimated primary completion date: 30 October 2019.
\end{tabular}

CFU: colony-forming unit; GRA: Global Response Assessment; HADS: Hospital Anxiety and Depression Scale; IIEF: International Index of Erectile Function; IPSS: International Prostate Symptom Score; NIH-CPSI: National Institutes of Health - Chronic Prostatitis Symptom Index; SAS: Self-rating Anxiety Scale; SDS: Self-rating Depression Scale.

\section{DATA AND ANALYSES}

\section{Comparison 1. Acupuncture versus sham procedure}

\begin{tabular}{|c|c|c|c|c|}
\hline Outcome or subgroup title & No. of studies & $\begin{array}{l}\text { No. of partici- } \\
\text { pants }\end{array}$ & Statistical method & Effect size \\
\hline $\begin{array}{l}1 \text { Prostatitis symptoms (NIH- } \\
\text { CPSI total) }\end{array}$ & 3 & 204 & Mean Difference (IV, Fixed, 95\% CI) & $-5.79[-7.32,-4.26]$ \\
\hline 1.1 Acupuncture & 2 & 180 & Mean Difference (IV, Fixed, 95\% CI) & $-5.71[-7.50,-3.91]$ \\
\hline 1.2 Electroacupuncture & 1 & 24 & Mean Difference (IV, Fixed, 95\% CI) & $-6.0[-8.92,-3.08]$ \\
\hline $\begin{array}{l}2 \text { Prostatitis symptoms: pain } \\
\text { subscore }\end{array}$ & 3 & 204 & Mean Difference (IV, Fixed, 95\% Cl) & $-2.43[-3.21,-1.66]$ \\
\hline 2.1 Acupuncture & 2 & 180 & Mean Difference (IV, Fixed, 95\% CI) & $-2.00[-2.90,-1.10]$ \\
\hline 2.2 Electroacupuncture & 1 & 24 & Mean Difference (IV, Fixed, 95\% CI) & $-3.6[-5.08,-2.12]$ \\
\hline $\begin{array}{l}3 \text { Prostatitis symptoms: mic- } \\
\text { turition subscore }\end{array}$ & 3 & 204 & Mean Difference (IV, Fixed, 95\% CI) & $-1.45[-1.83,-1.06]$ \\
\hline 3.1 Acupuncture & 2 & 180 & Mean Difference (IV, Fixed, 95\% CI) & $-1.49[-1.89,-1.08]$ \\
\hline 3.2 Electroacupuncture & 1 & 24 & Mean Difference (IV, Fixed, 95\% CI) & $-1.1[-2.26,0.06]$ \\
\hline $\begin{array}{l}4 \text { Prostatitis symptoms: qual- } \\
\text { ity of life subscore }\end{array}$ & 3 & 204 & Mean Difference (IV, Fixed, 95\% CI) & $-2.40[-3.09,-1.71]$ \\
\hline 4.1 Acupuncture & 2 & 180 & Mean Difference (IV, Fixed, 95\% CI) & $-2.62[-3.37,-1.86]$ \\
\hline 4.2 Electroacupuncture & 1 & 24 & Mean Difference (IV, Fixed, 95\% CI) & $-1.3[-1.00,0.40]$ \\
\hline 5 Prostatitis symptoms & 2 & 113 & Risk Ratio (M-H, Random, 95\% Cl) & $2.49[0.77,8.02]$ \\
\hline 5.1 Acupuncture & 1 & 89 & Risk Ratio (M-H, Random, 95\% Cl) & $1.56[1.09,2.24]$ \\
\hline 5.2 Electroacupuncture & 1 & 24 & Risk Ratio (M-H, Random, 95\% Cl) & $5.00[1.63,15.31]$ \\
\hline
\end{tabular}




\begin{tabular}{|c|c|c|c|c|}
\hline Outcome or subgroup title & No. of studies & $\begin{array}{l}\text { No. of partici- } \\
\text { pants }\end{array}$ & Statistical method & Effect size \\
\hline $\begin{array}{l}6 \text { Prostatitis symptoms (NIH- } \\
\text { CPSI total) - medium term }\end{array}$ & 1 & 91 & Mean Difference (IV, Fixed, 95\% CI) & $-7.36[-9.93,-4.79]$ \\
\hline $\begin{array}{l}7 \text { Prostatitis symptoms: pain } \\
\text { subscore - medium term }\end{array}$ & 1 & 91 & Mean Difference (IV, Fixed, 95\% CI) & $-3.25[-4.45,-2.05]$ \\
\hline $\begin{array}{l}8 \text { Prostatitis symptoms: mic- } \\
\text { turition subscore - medium } \\
\text { term }\end{array}$ & 1 & 91 & Mean Difference (IV, Fixed, 95\% CI) & $-1.02[-1.54,-0.50]$ \\
\hline $\begin{array}{l}9 \text { Prostatitis symptoms: qual- } \\
\text { ity of life subscore - medium } \\
\text { term }\end{array}$ & 1 & 91 & Mean Difference (IV, Fixed, 95\% CI) & $-3.07[-4.14,-2.00]$ \\
\hline 10 Adverse events & 3 & 204 & Risk Ratio (M-H, Fixed, 95\% Cl) & $1.33[0.51,3.46]$ \\
\hline 10.1 Acupuncture & 2 & 180 & Risk Ratio (M-H, Fixed, 95\% Cl) & $1.64[0.58,4.62]$ \\
\hline 10.2 Electroacupuncture & 1 & 24 & Risk Ratio (M-H, Fixed, 95\% Cl) & $0.33[0.01,7.45]$ \\
\hline 11 Sexual dysfunction & 1 & 89 & Mean Difference (IV, Fixed, 95\% CI) & $-0.5[-3.46,2.46]$ \\
\hline 12 Urinary symptoms & 2 & 113 & Mean Difference (IV, Fixed, 95\% CI) & $-2.79[-4.77,-0.82]$ \\
\hline 12.1 Acupuncture & 1 & 89 & Mean Difference (IV, Fixed, 95\% CI) & $-2.5[-4.98,-0.02]$ \\
\hline 12.2 Electroacupuncture & 1 & 24 & Mean Difference (IV, Fixed, 95\% CI) & $-3.30[-6.56,-0.04]$ \\
\hline
\end{tabular}

Analysis 1.1. Comparison 1 Acupuncture versus sham procedure, Outcome 1 Prostatitis symptoms (NIH-CPSI total).

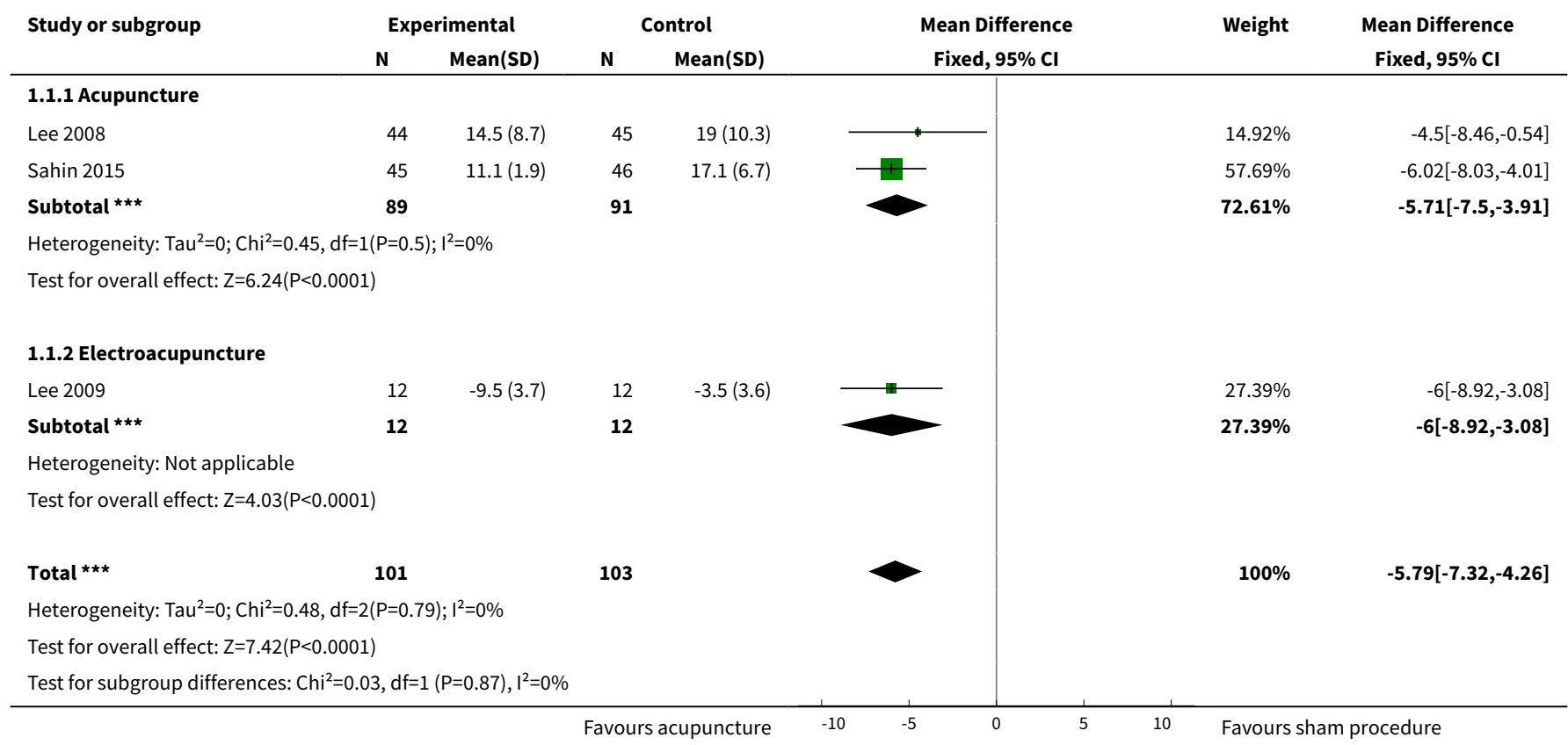


Analysis 1.2. Comparison 1 Acupuncture versus sham procedure, Outcome 2 Prostatitis symptoms: pain subscore.

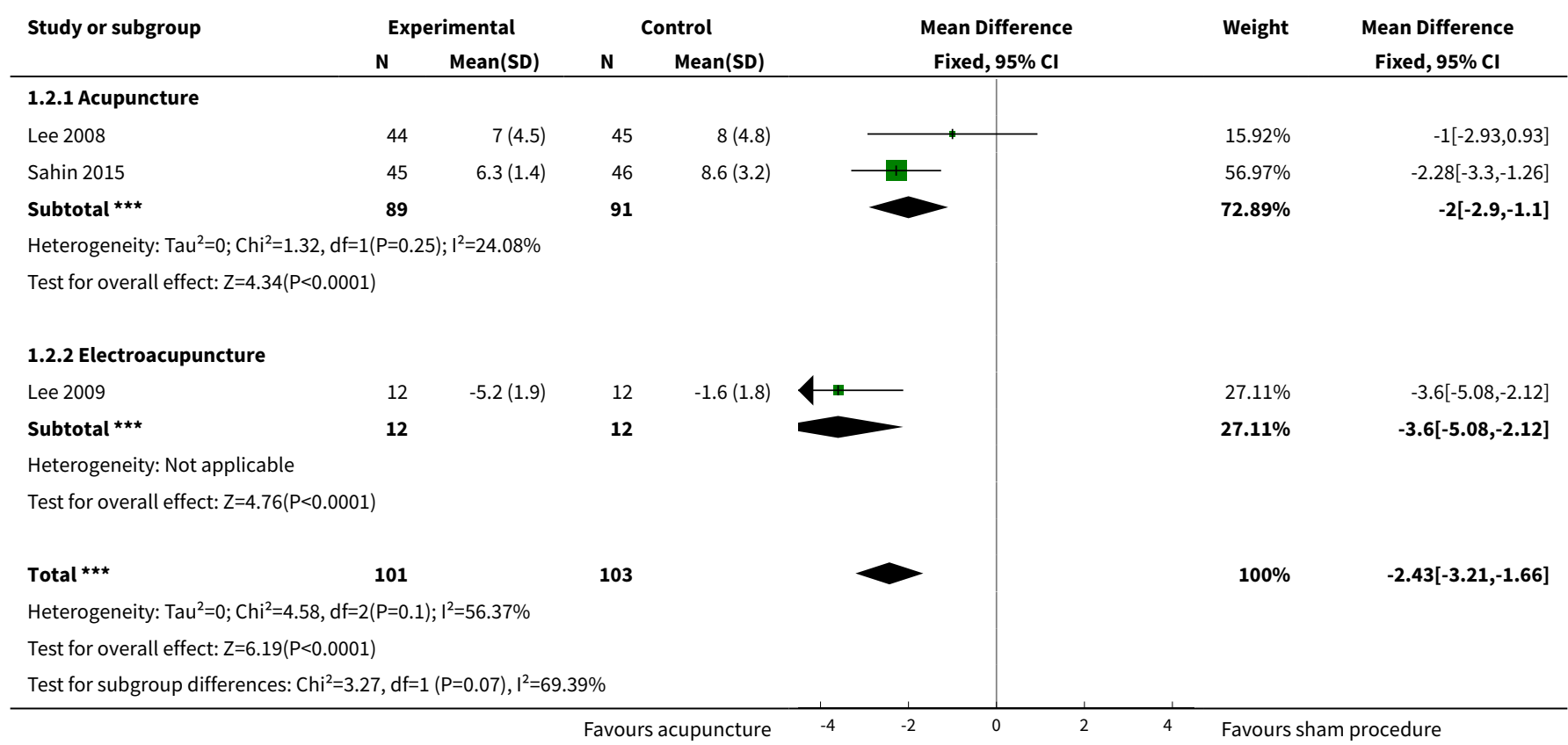

\section{Analysis 1.3. Comparison 1 Acupuncture versus sham procedure,} Outcome 3 Prostatitis symptoms: micturition subscore.

\begin{tabular}{|c|c|c|c|c|c|c|c|}
\hline \multirow[t]{2}{*}{ Study or subgroup } & \multicolumn{2}{|c|}{ Experimental } & \multicolumn{2}{|c|}{ Control } & \multirow{2}{*}{$\begin{array}{c}\text { Mean Difference } \\
\text { Fixed, } 95 \% \mathrm{Cl}\end{array}$} & \multirow[t]{2}{*}{ Weight } & \multirow{2}{*}{$\begin{array}{c}\text { Mean Difference } \\
\text { Fixed, } 95 \% \mathrm{Cl}\end{array}$} \\
\hline & $\mathbf{N}$ & $\operatorname{Mean}(S D)$ & $\mathbf{N}$ & $\operatorname{Mean}(S D)$ & & & \\
\hline \multicolumn{8}{|l|}{ 1.3.1 Acupuncture } \\
\hline Lee 2008 & 44 & $0(2.9)$ & 45 & $2(3.2)$ & $*$ & $9.08 \%$ & $-2[-3.27,-0.73]$ \\
\hline Sahin 2015 & 45 & $2.2(0.8)$ & 46 & $3.7(1.3)$ & & $80.08 \%$ & $-1.43[-1.86,-1]$ \\
\hline Subtotal $* \star \star$ & 89 & & 91 & & & $89.16 \%$ & $-1.49[-1.89,-1.08]$ \\
\hline \multicolumn{8}{|c|}{ Heterogeneity: $\mathrm{Tau}^{2}=0 ; \mathrm{Chi}^{2}=0.7, \mathrm{df}=1(\mathrm{P}=0.4) ; \mathrm{I}^{2}=0 \%$} \\
\hline \multicolumn{8}{|c|}{ 1.3.2 Electroacupuncture } \\
\hline Lee 2009 & 12 & $-1.8(1.5)$ & 12 & $-0.7(1.4)$ & + & $10.84 \%$ & $-1.1[-2.26,0.06]$ \\
\hline Subtotal $\star \star \star$ & 12 & & 12 & & 1 & $10.84 \%$ & $-1.1[-2.26,0.06]$ \\
\hline \multicolumn{8}{|c|}{ Heterogeneity: Not applicable } \\
\hline \multicolumn{8}{|c|}{ Test for overall effect: $Z=1.86(P=0.06)$} \\
\hline 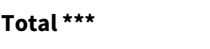 & 101 & & 103 & & & $100 \%$ & $-1.45[-1.83,-1.06]$ \\
\hline \multicolumn{8}{|c|}{ Heterogeneity: $\operatorname{Tau}^{2}=0 ; \mathrm{Chi}^{2}=1.08, \mathrm{df}=2(\mathrm{P}=0.58) ; \mathrm{I}^{2}=0 \%$} \\
\hline \multicolumn{8}{|c|}{ Test for overall effect: $Z=7.42(P<0.0001)$} \\
\hline Test for subgroup dif & $38, \mathrm{df}=$ & $P=0.54), I^{2}=0 \%$ & & & & & \\
\hline
\end{tabular}




\section{Analysis 1.4. Comparison 1 Acupuncture versus sham procedure,} Outcome 4 Prostatitis symptoms: quality of life subscore.

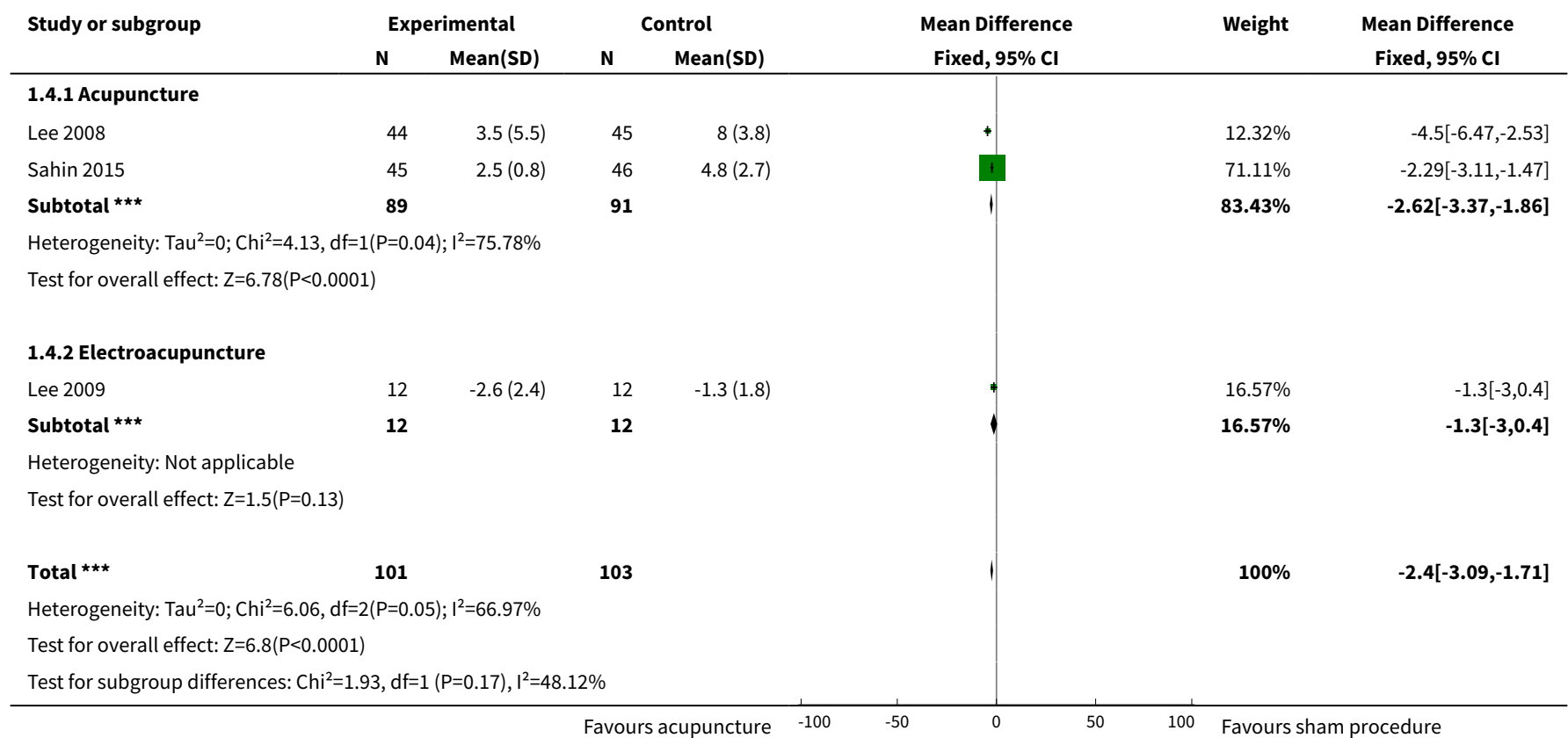

\section{Analysis 1.5. Comparison 1 Acupuncture versus sham procedure, Outcome 5 Prostatitis symptoms.}

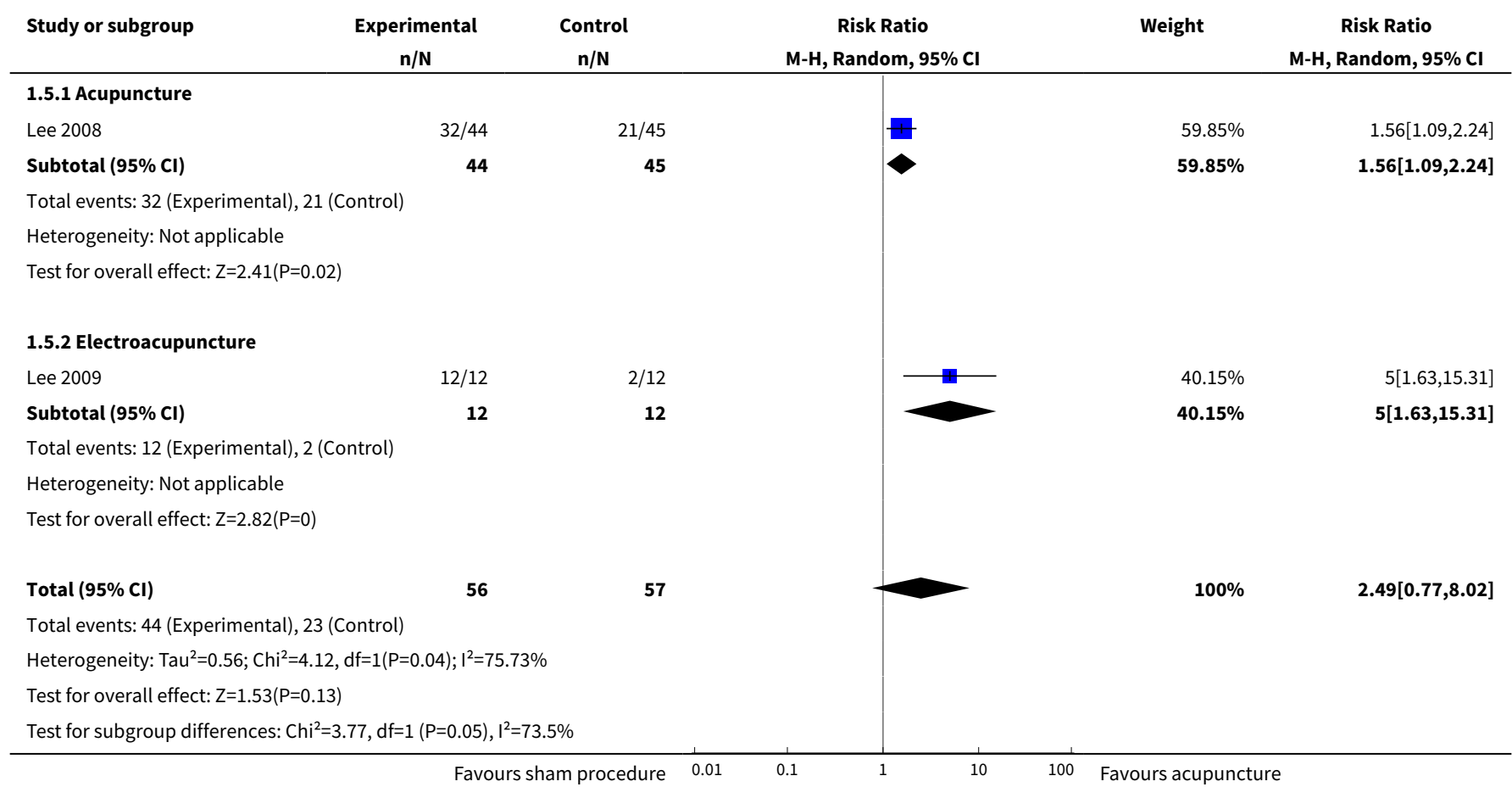


Analysis 1.6. Comparison 1 Acupuncture versus sham procedure, Outcome 6 Prostatitis symptoms (NIH-CPSI total) - medium term.

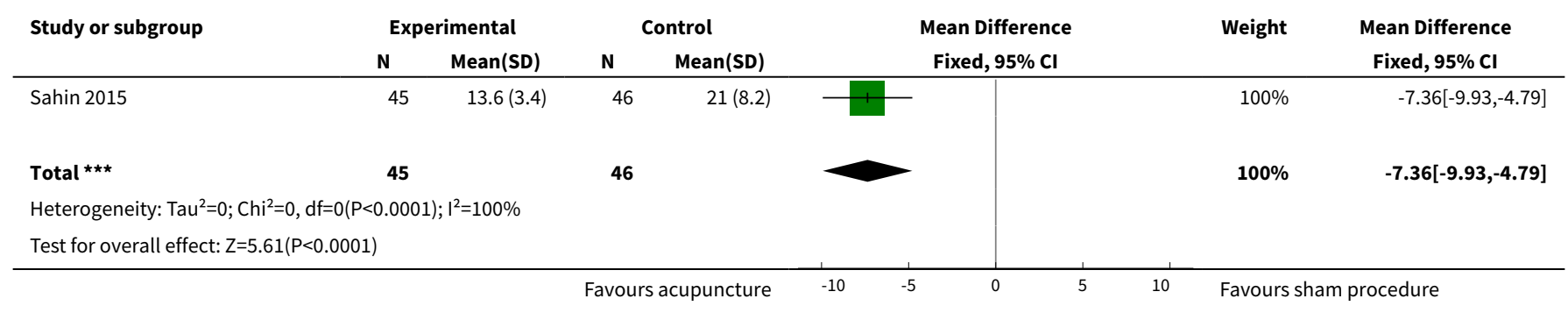

Analysis 1.7. Comparison 1 Acupuncture versus sham procedure,

Outcome 7 Prostatitis symptoms: pain subscore - medium term.

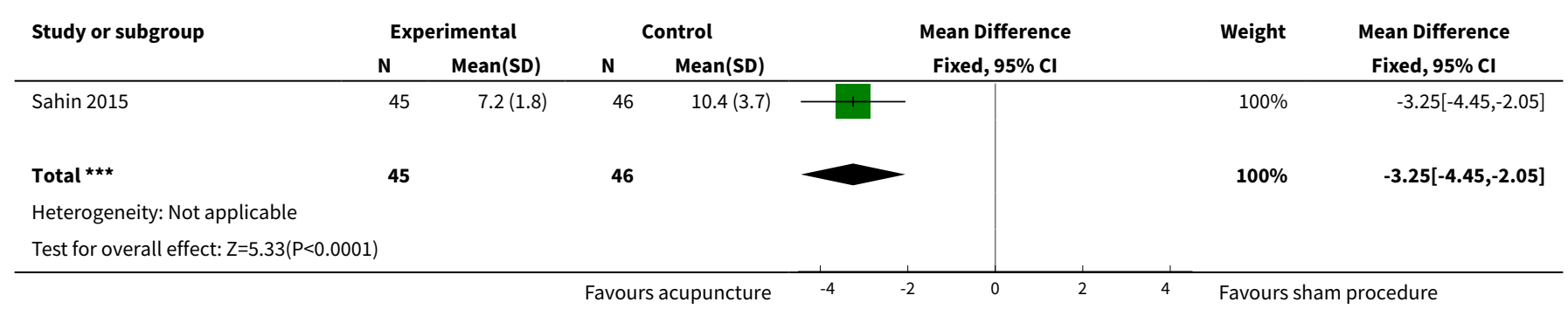

Analysis 1.8. Comparison 1 Acupuncture versus sham procedure, Outcome 8 Prostatitis symptoms: micturition subscore - medium term.

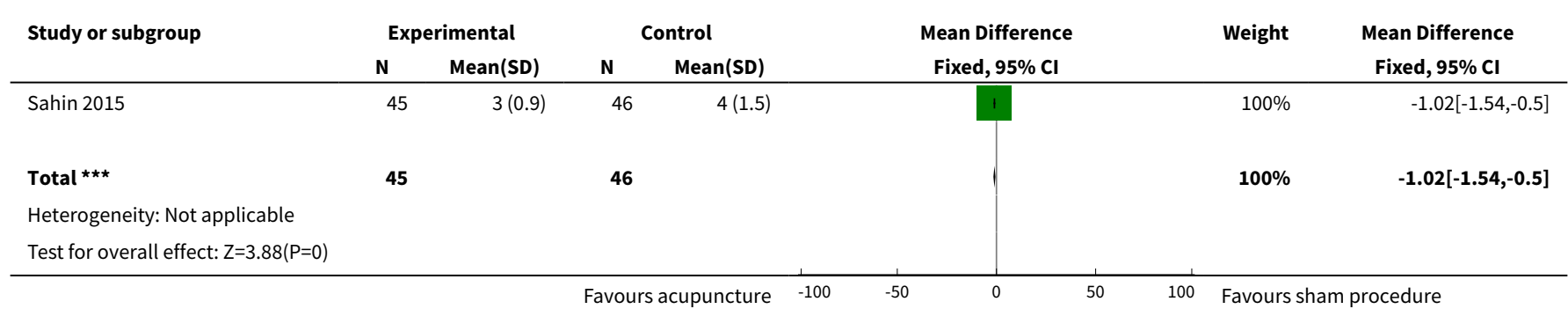

Analysis 1.9. Comparison 1 Acupuncture versus sham procedure, Outcome 9 Prostatitis symptoms: quality of life subscore - medium term.

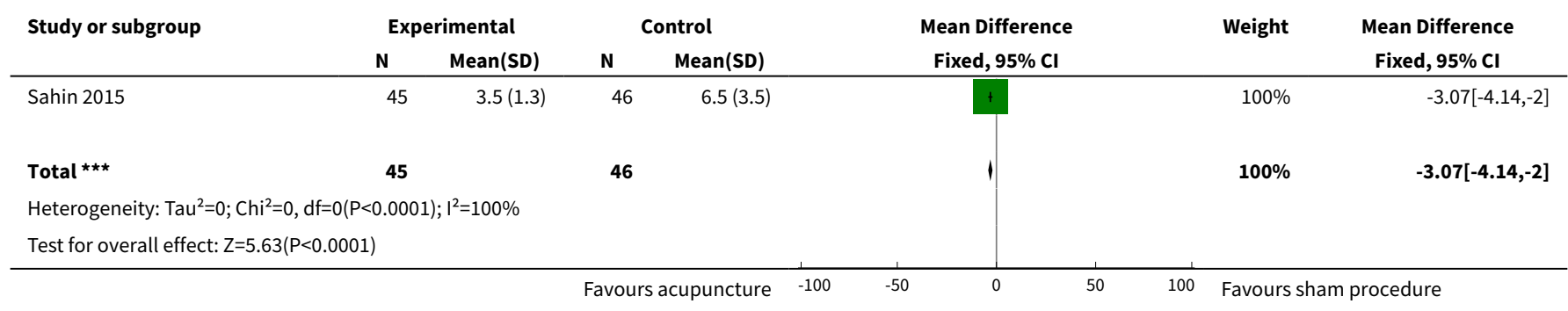


Analysis 1.10. Comparison 1 Acupuncture versus sham procedure, Outcome 10 Adverse events.

\begin{tabular}{|c|c|c|c|c|c|}
\hline Study or subgroup & $\begin{array}{c}\text { Experimental } \\
n / N\end{array}$ & $\begin{array}{c}\text { Control } \\
n / N\end{array}$ & $\begin{array}{c}\text { Risk Ratio } \\
\text { M-H, Fixed, } 95 \% \mathrm{Cl} \\
\end{array}$ & Weight & $\begin{array}{c}\text { Risk Ratio } \\
\text { M-H, Fixed, } 95 \% \mathrm{Cl} \\
\end{array}$ \\
\hline \multicolumn{6}{|l|}{ 1.10.1 Acupuncture } \\
\hline Lee 2008 & $8 / 44$ & $5 / 45$ & & $76.72 \%$ & $1.64[0.58,4.62]$ \\
\hline Sahin 2015 & $0 / 45$ & $0 / 46$ & & & Not estimable \\
\hline Subtotal (95\% Cl) & 89 & 91 & & $76.72 \%$ & $1.64[0.58,4.62]$ \\
\hline \multicolumn{6}{|c|}{ Total events: 8 (Experimental), 5 (Control) } \\
\hline \multicolumn{6}{|c|}{ Heterogeneity: Not applicable } \\
\hline \multicolumn{6}{|c|}{ Test for overall effect: $Z=0.93(P=0.35)$} \\
\hline \multicolumn{6}{|c|}{ 1.10.2 Electroacupuncture } \\
\hline Lee 2009 & $0 / 12$ & $1 / 12$ & & $23.28 \%$ & $0.33[0.01,7.45]$ \\
\hline Subtotal $(95 \% \mathrm{Cl})$ & 12 & 12 & & $23.28 \%$ & $0.33[0.01,7.45]$ \\
\hline \multicolumn{6}{|c|}{ Total events: 0 (Experimental), 1 (Control) } \\
\hline \multicolumn{6}{|c|}{ Heterogeneity: Not applicable } \\
\hline \multicolumn{6}{|c|}{ Test for overall effect: $\mathrm{Z}=0.69(\mathrm{P}=0.49)$} \\
\hline Total $(95 \% \mathrm{Cl})$ & 101 & 103 & & $100 \%$ & $1.33[0.51,3.46]$ \\
\hline \multicolumn{6}{|c|}{ Total events: 8 (Experimental), 6 (Control) } \\
\hline \multicolumn{6}{|c|}{ Heterogeneity: Tau $^{2}=0 ; \mathrm{Chi}^{2}=0.91, \mathrm{df}=1(\mathrm{P}=0.34) ; \mathrm{I}^{2}=0 \%$} \\
\hline \multicolumn{6}{|c|}{ Test for overall effect: $\mathrm{Z}=0.59(\mathrm{P}=0.55)$} \\
\hline \multicolumn{6}{|c|}{ Test for subgroup differences: $\mathrm{Chi}^{2}=0.91, \mathrm{df}=1(\mathrm{P}=0.34), \mathrm{I}^{2}=0 \%$} \\
\hline
\end{tabular}

Analysis 1.11. Comparison 1 Acupuncture versus sham procedure, Outcome 11 Sexual dysfunction.

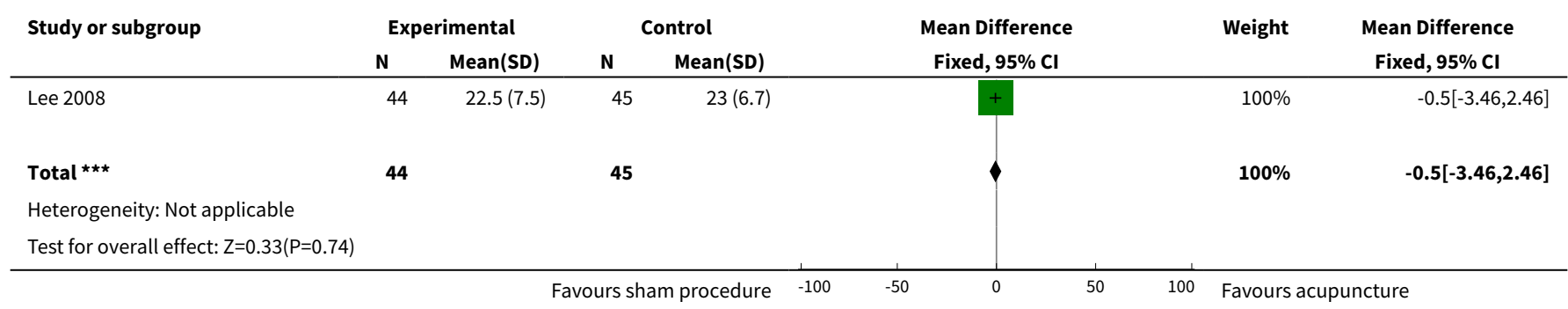

Analysis 1.12. Comparison 1 Acupuncture versus sham procedure, Outcome 12 Urinary symptoms.

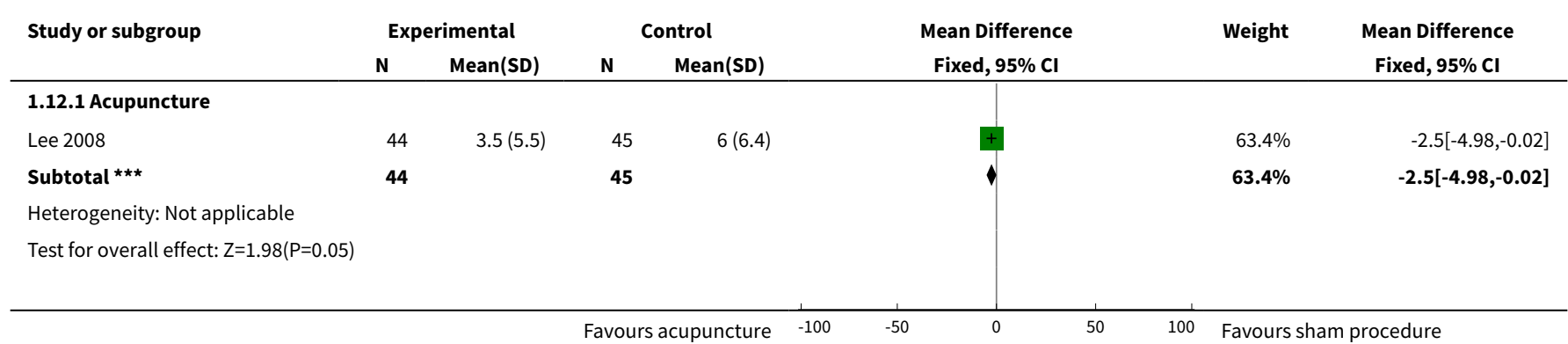




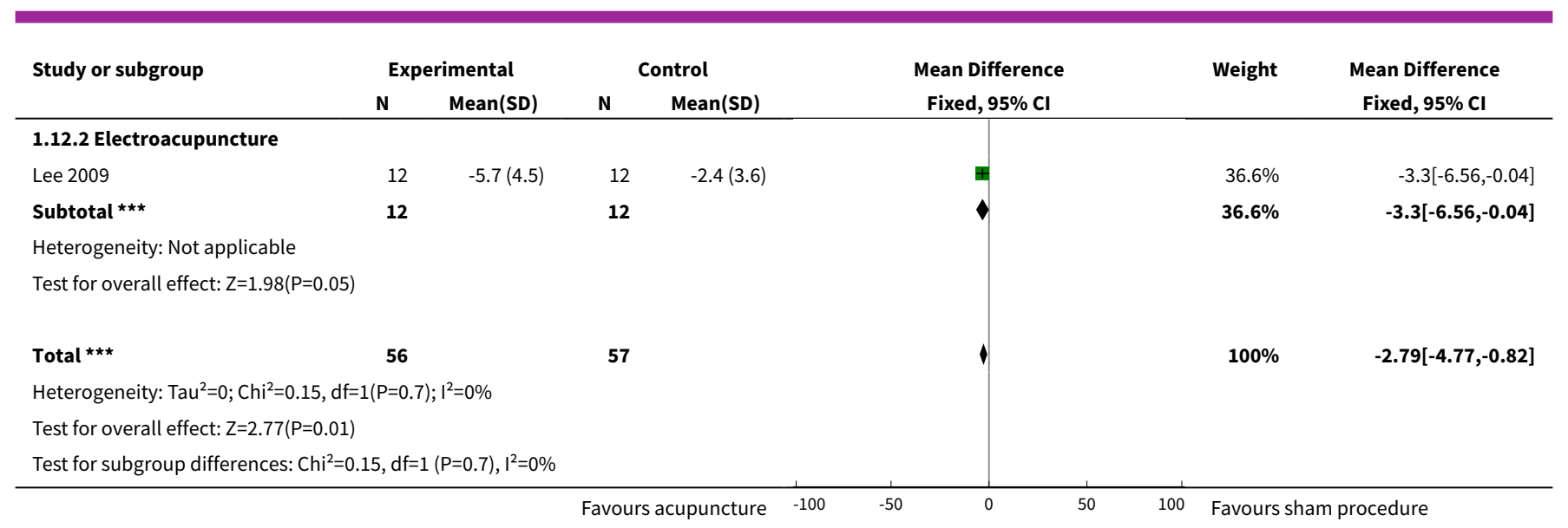

\section{Comparison 2. Acupuncture treatments versus medical treatment}

\begin{tabular}{|c|c|c|c|c|}
\hline $\begin{array}{l}\text { Outcome or subgroup ti- } \\
\text { tle }\end{array}$ & No. of studies & $\begin{array}{l}\text { No. of partici- } \\
\text { pants }\end{array}$ & Statistical method & Effect size \\
\hline $\begin{array}{l}1 \text { Prostatitis symptoms } \\
\text { (NIH-CPSI total) }\end{array}$ & 3 & 203 & $\begin{array}{l}\text { Mean Difference (IV, Random, 95\% } \\
\mathrm{CI})\end{array}$ & $-4.09[-6.87,-1.30]$ \\
\hline 1.1 Acupuncture & 2 & 116 & $\begin{array}{l}\text { Mean Difference (IV, Random, 95\% } \\
\text { CI) }\end{array}$ & $-2.97[-9.36,3.43]$ \\
\hline 1.2 Electroacupuncture & 1 & 24 & $\begin{array}{l}\text { Mean Difference (IV, Random, 95\% } \\
\text { CI) }\end{array}$ & $-6.0[-8.50,-3.50]$ \\
\hline $\begin{array}{l}\text { 1.3 Acupuncture (Moxibus- } \\
\text { tion) }\end{array}$ & 1 & 63 & $\begin{array}{l}\text { Mean Difference (IV, Random, 95\% } \\
\text { CI) }\end{array}$ & $-3.74[-7.41,-0.07]$ \\
\hline $\begin{array}{l}2 \text { Prostatitis symptoms: } \\
\text { pain subscore }\end{array}$ & 2 & 78 & Mean Difference (IV, Fixed, 95\% CI) & $-2.90[-4.05,-1.76]$ \\
\hline 2.1 Acupuncture & 1 & 54 & Mean Difference (IV, Fixed, 95\% Cl) & $-2.76[-4.57,-0.95]$ \\
\hline 2.2 Electroacupuncture & 1 & 24 & Mean Difference (IV, Fixed, 95\% CI) & $-3.00[-4.48,-1.52]$ \\
\hline $\begin{array}{l}3 \text { Prostatitis symptoms: } \\
\text { micturition subscore }\end{array}$ & 2 & 78 & Mean Difference (IV, Fixed, 95\% CI) & $-1.15[-1.96,-0.35]$ \\
\hline 3.1 Acupuncture & 1 & 54 & Mean Difference (IV, Fixed, 95\% CI) & $-0.95[-2.03,0.13]$ \\
\hline 3.2 Electroacupuncture & 1 & 24 & Mean Difference (IV, Fixed, 95\% CI) & $-1.4[-2.60,-0.20]$ \\
\hline $\begin{array}{l}4 \text { Prostatitis symptoms: } \\
\text { quality of life subscore }\end{array}$ & 2 & 78 & Mean Difference (IV, Fixed, 95\% CI) & $-1.41[-2.41,-0.41]$ \\
\hline 4.1 Acupuncture & 1 & 54 & Mean Difference (IV, Fixed, 95\% CI) & $-1.18[-2.52,0.16]$ \\
\hline 4.2 Electroacupuncture & 1 & 24 & Mean Difference (IV, Fixed, 95\% CI) & $-1.70[-3.19,-0.21]$ \\
\hline 5 Prostatitis symptoms & 1 & 24 & Risk Ratio (M-H, Fixed, 95\% Cl) & $3.57[1.45,8.80]$ \\
\hline
\end{tabular}




\begin{tabular}{lllll}
\hline $\begin{array}{l}\text { Outcome or subgroup ti- } \\
\text { tle }\end{array}$ & No. of studies & $\begin{array}{l}\text { No. of partici- } \\
\text { pants }\end{array}$ & Statistical method & Effect size \\
\hline 5.1 Electroacupuncture & 1 & 24 & Risk Ratio (M-H, Fixed, 95\% Cl) & $3.57[1.45,8.80]$ \\
\hline 6 Adverse events & 2 & & Risk Ratio $(\mathrm{M}-\mathrm{H}$, Fixed, 95\% Cl) & Totals not selected \\
\hline 6.1 Acupuncture & 1 & & Risk Ratio (M-H, Fixed, 95\% Cl) & $0.0[0.0,0.0]$ \\
\hline 6.2 Electroacupuncture & 1 & & Risk Ratio (M-H, Fixed, 95\% Cl) & $0.0[0.0,0.0]$ \\
\hline 7 Urinary symptoms & 1 & 24 & Mean Difference (IV, Fixed, 95\% Cl) & $-2.7[-4.00,0.60]$ \\
\hline 7.1 Electroacupuncture & 1 & 24 & Mean Difference (IV, Fixed, 95\% Cl) & $-2.7[-4.00,0.60]$ \\
\hline
\end{tabular}

\section{Analysis 2.1. Comparison 2 Acupuncture treatments versus medical treatment, Outcome 1 Prostatitis symptoms (NIH-CPSI total).}

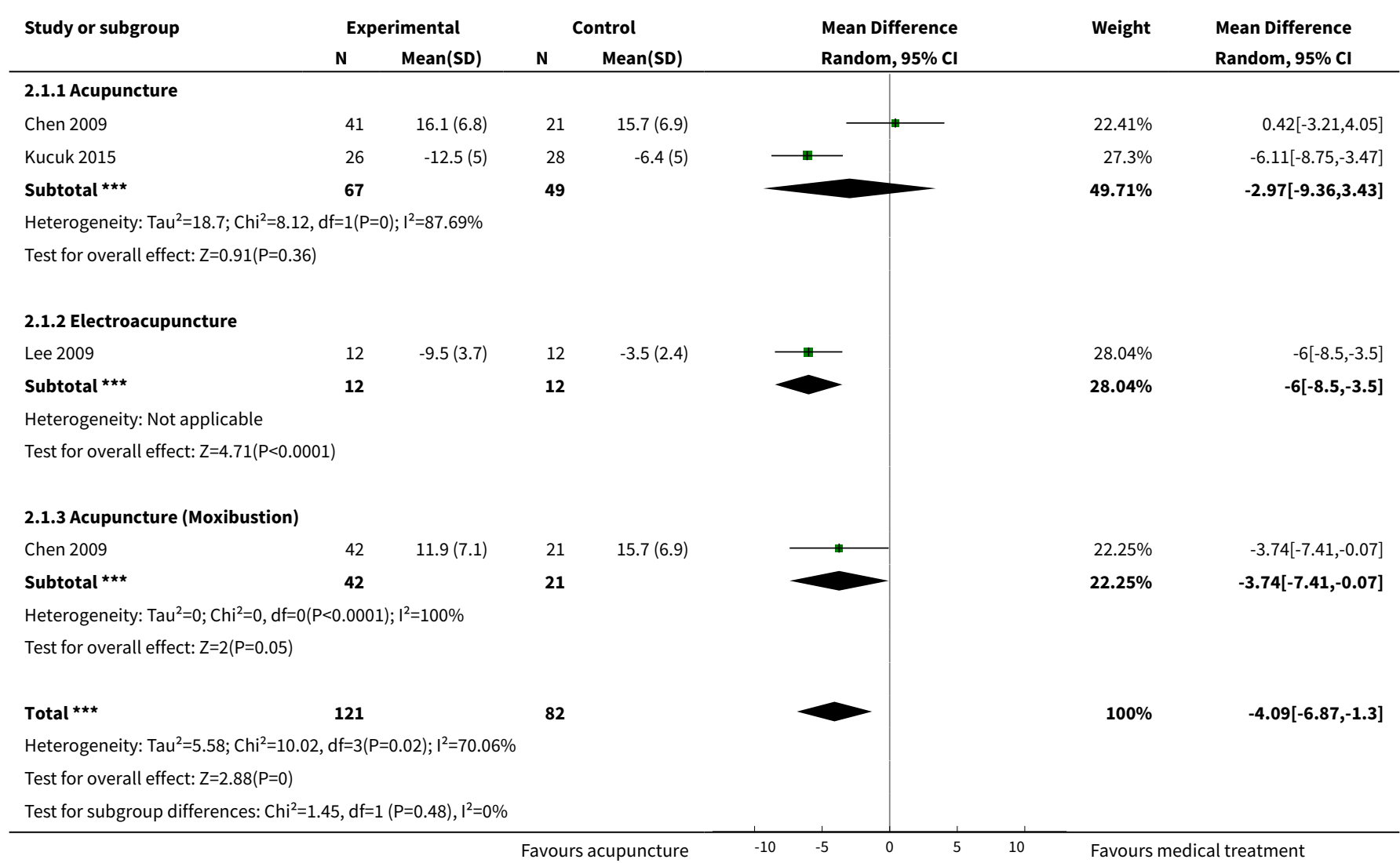


Analysis 2.2. Comparison 2 Acupuncture treatments versus medical treatment, Outcome 2 Prostatitis symptoms: pain subscore.

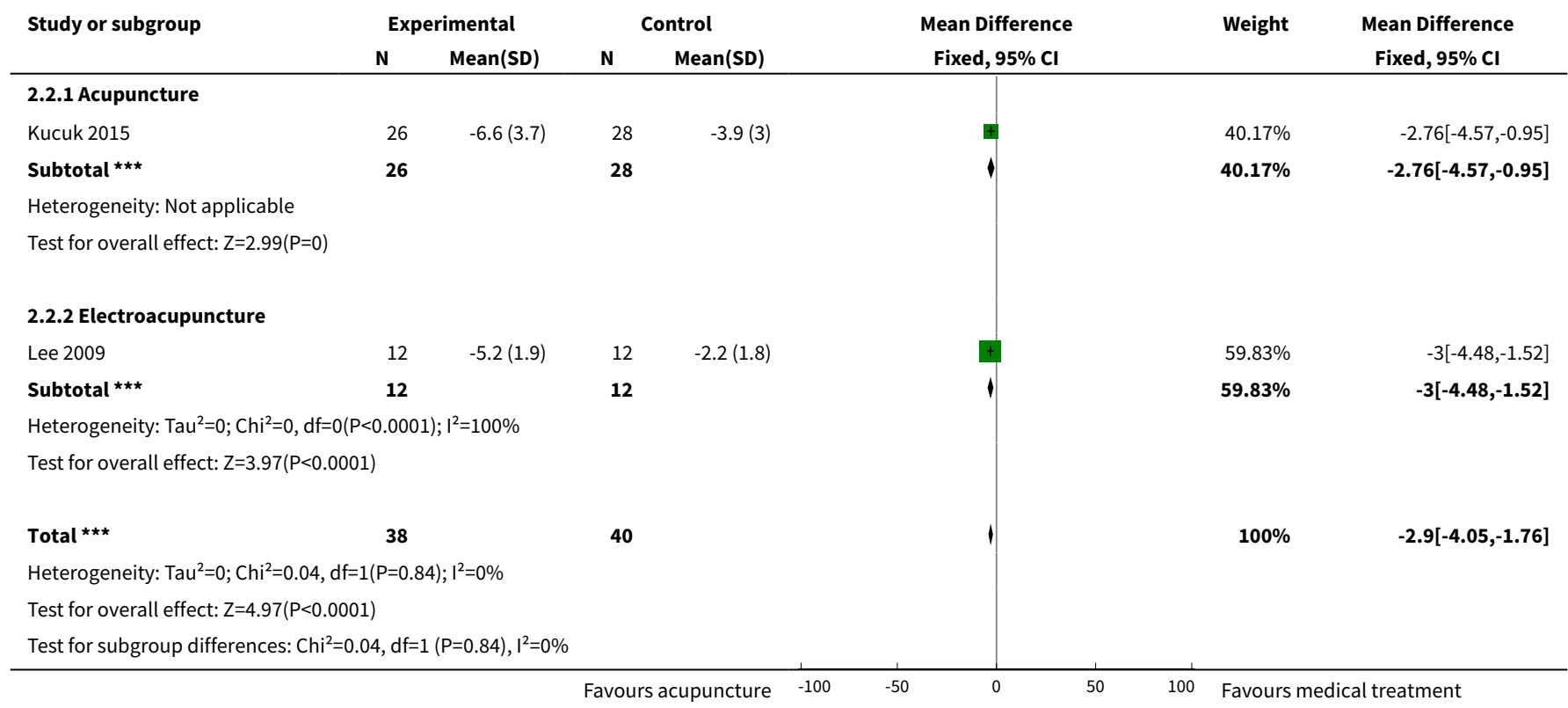

\section{Analysis 2.3. Comparison 2 Acupuncture treatments versus medical treatment, Outcome 3 Prostatitis symptoms: micturition subscore.}

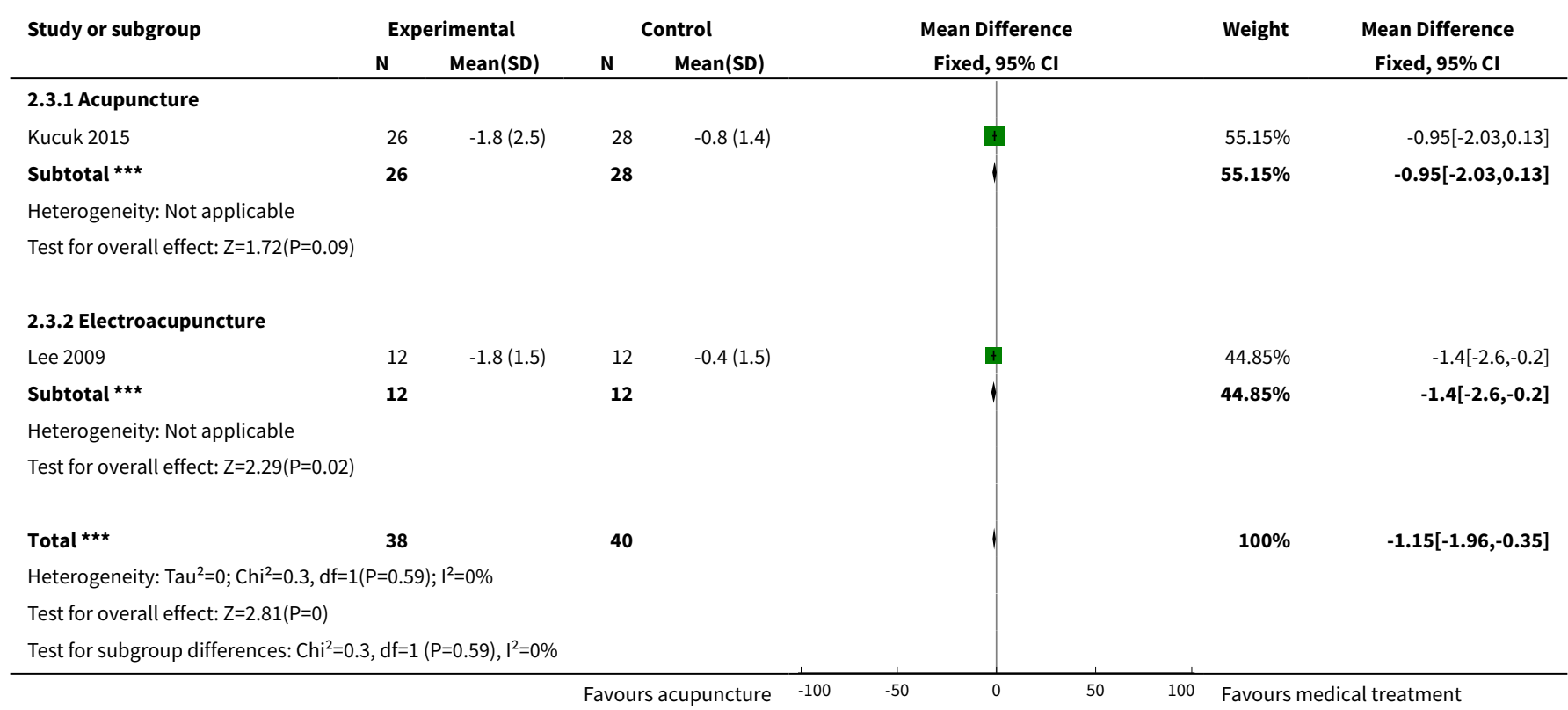


Analysis 2.4. Comparison 2 Acupuncture treatments versus medical treatment, Outcome 4 Prostatitis symptoms: quality of life subscore.

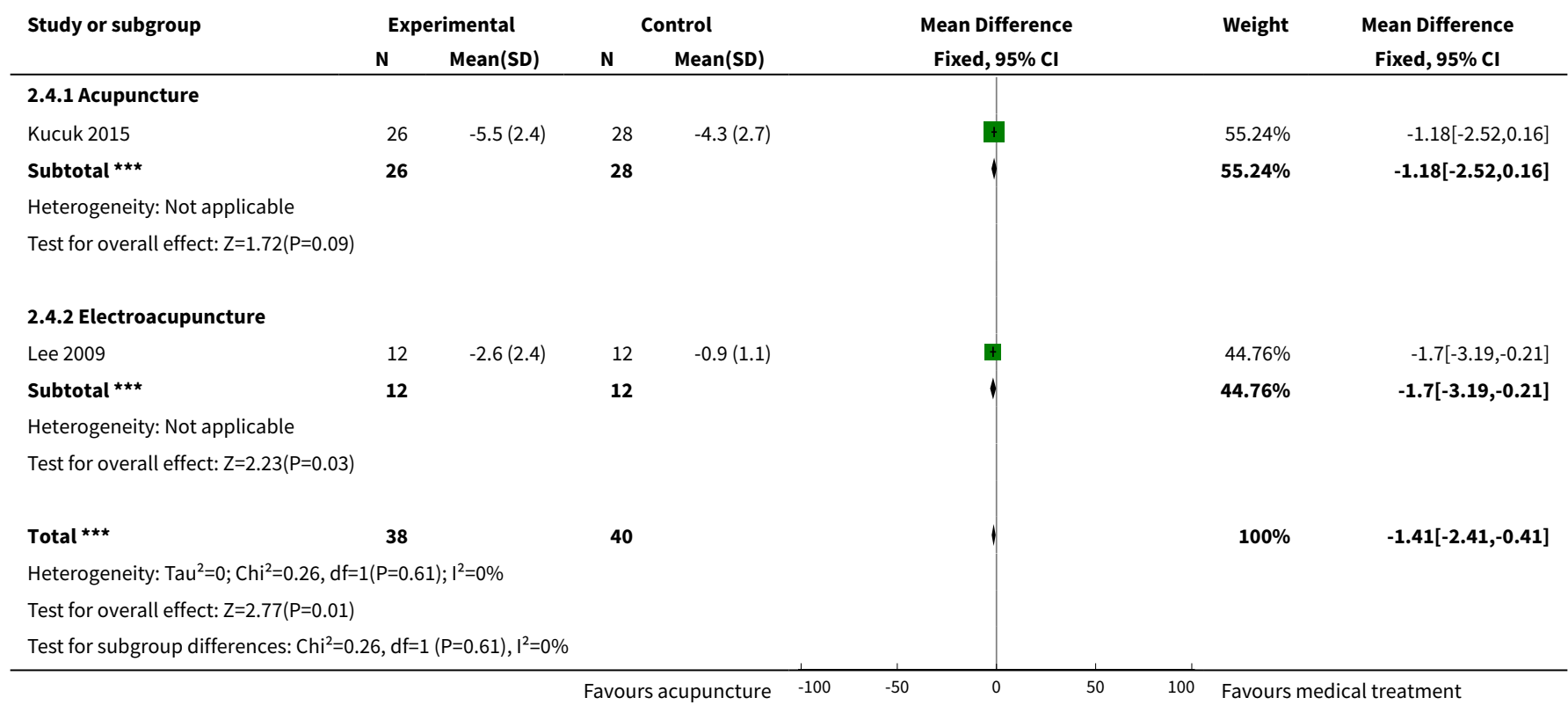

Analysis 2.5. Comparison 2 Acupuncture treatments versus medical treatment, Outcome 5 Prostatitis symptoms.

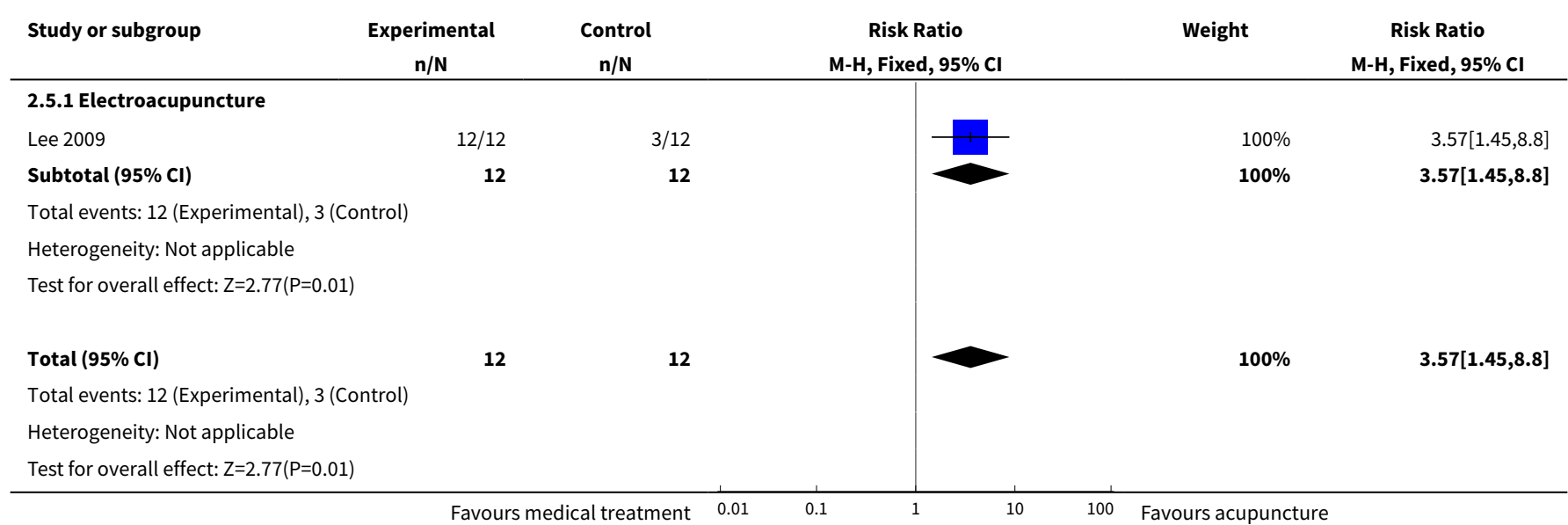

Analysis 2.6. Comparison 2 Acupuncture treatments versus medical treatment, Outcome 6 Adverse events.

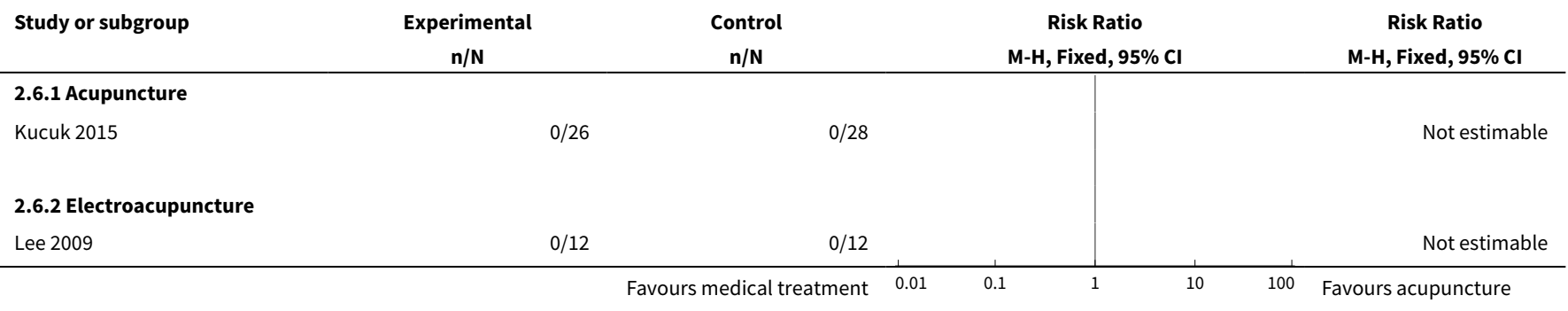


Analysis 2.7. Comparison 2 Acupuncture treatments versus medical treatment, Outcome 7 Urinary symptoms.

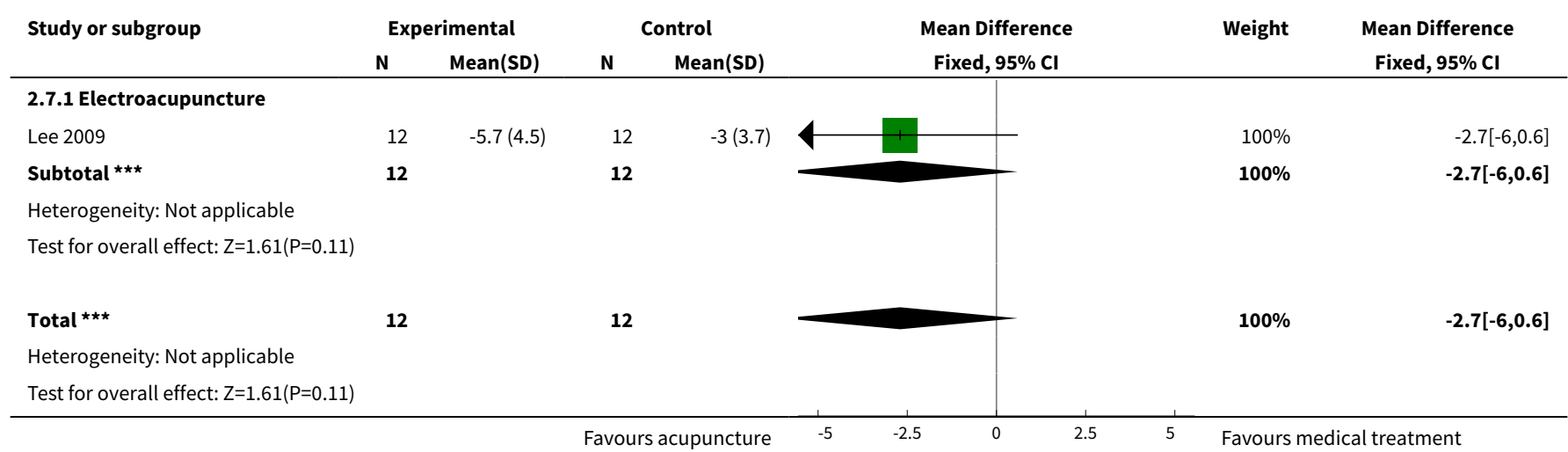

\section{Comparison 3. Acupuncture with or without moxibustion}

\begin{tabular}{lllll}
\hline Outcome or subgroup title & No. of studies & $\begin{array}{l}\text { No. of partici- } \\
\text { pants }\end{array}$ & Statistical method & Effect size \\
\hline $\begin{array}{l}1 \text { Prostatitis symptoms (NIH-CPSI to- } \\
\text { tal) }\end{array}$ & 1 & 83 & $\begin{array}{l}\text { Mean Difference (IV, Fixed, } \\
95 \% \mathrm{Cl})\end{array}$ & $-4.16[-7.16,-1.16]$ \\
\hline
\end{tabular}

Analysis 3.1. Comparison 3 Acupuncture with or without moxibustion, Outcome 1 Prostatitis symptoms (NIH-CPSI total).

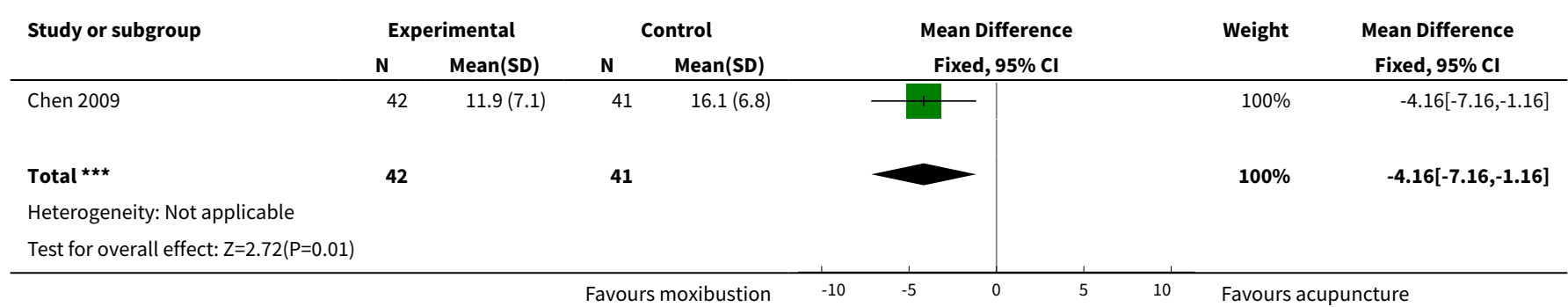

\section{Comparison 4. Circumcision versus waiting list}

\begin{tabular}{|c|c|c|c|c|}
\hline Outcome or subgroup title & No. of studies & $\begin{array}{l}\text { No. of partici- } \\
\text { pants }\end{array}$ & Statistical method & Effect size \\
\hline 1 Prostatitis symptoms & 1 & 713 & $\begin{array}{l}\text { Mean Difference (IV, Fixed, 95\% } \\
\mathrm{CI} \text { ) }\end{array}$ & $-3.0[-3.82,-2.18]$ \\
\hline $\begin{array}{l}2 \text { Prostatitis symptoms: pain sub- } \\
\text { score }\end{array}$ & 1 & 713 & $\begin{array}{l}\text { Mean Difference (IV, Fixed, 95\% } \\
\mathrm{CI} \text { ) }\end{array}$ & $-1.0[-1.33,-0.67]$ \\
\hline
\end{tabular}




\begin{tabular}{lllll}
\hline Outcome or subgroup title & No. of studies & $\begin{array}{l}\text { No. of partici- } \\
\text { pants }\end{array}$ & Statistical method & Effect size \\
\hline $\begin{array}{l}\text { 3 Prostatitis symptoms: micturi- } \\
\text { tion subscore }\end{array}$ & 1 & 713 & $\begin{array}{l}\text { Mean Difference (IV, Fixed, 95\% } \\
\text { CI) }\end{array}$ & $-1.0[-1.22,-0.78]$ \\
\hline $\begin{array}{l}\text { 4 Prostatitis symptoms: quality of } \\
\text { life subscore }\end{array}$ & 1 & 713 & $\begin{array}{l}\text { Mean Difference (IV, Fixed, 95\% } \\
\text { Cl) }\end{array}$ & $-3.0[-3.38,-2.62]$ \\
\hline 5 Adverse events & 1 & 713 & Risk Ratio (M-H, Fixed, 95\% Cl) & 1.23 [0.86, 1.76] \\
\hline
\end{tabular}

Analysis 4.1. Comparison 4 Circumcision versus waiting list, Outcome 1 Prostatitis symptoms.

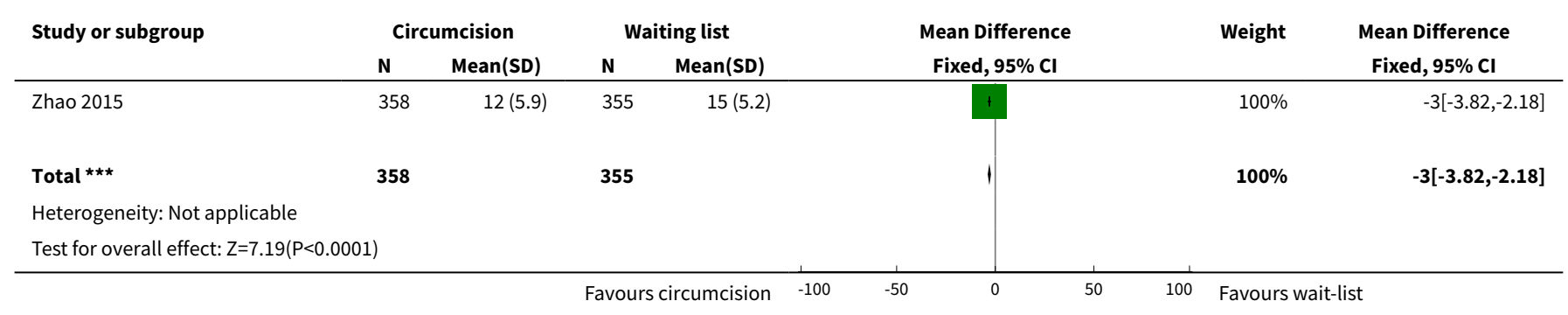

Analysis 4.2. Comparison 4 Circumcision versus waiting list, Outcome 2 Prostatitis symptoms: pain subscore.

\begin{tabular}{|c|c|c|c|c|c|c|c|c|}
\hline \multirow{3}{*}{$\begin{array}{l}\text { Study or subgroup } \\
\text { Zhao } 2015\end{array}$} & \multicolumn{2}{|c|}{ Circumcision } & \multicolumn{2}{|c|}{ Waiting list } & & \multirow{2}{*}{$\begin{array}{c}\text { Mean Difference } \\
\text { Fixed, } 95 \% \mathrm{Cl}\end{array}$} & \multirow[t]{2}{*}{ Weight } & \multirow{2}{*}{$\begin{array}{c}\text { Mean Difference } \\
\text { Fixed, } 95 \% \mathrm{Cl}\end{array}$} \\
\hline & $\mathbf{N}$ & $\operatorname{Mean}(S D)$ & $\mathbf{N}$ & Mean(SD) & & & & \\
\hline & 358 & $5(2.2)$ & 355 & $6(2.2)$ & & & $100 \%$ & $-1[-1.33,-0.67]$ \\
\hline 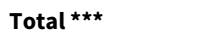 & 358 & & 355 & & & & $100 \%$ & $-1[-1.33,-0.67]$ \\
\hline \multicolumn{9}{|c|}{ Heterogeneity: Not applicable } \\
\hline \multicolumn{9}{|c|}{ Test for overall effect: $Z=6.01(P<0.0001)$} \\
\hline
\end{tabular}

Analysis 4.3. Comparison 4 Circumcision versus waiting list, Outcome 3 Prostatitis symptoms: micturition subscore.

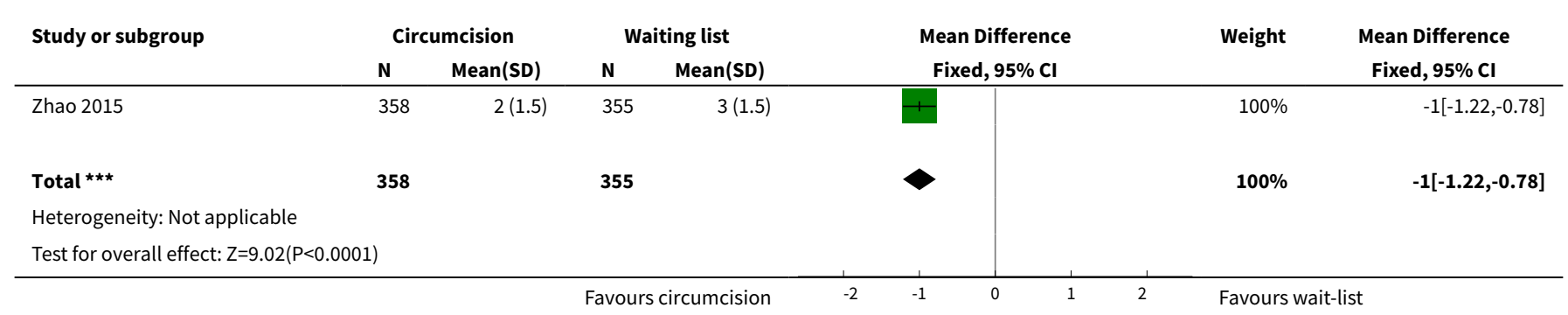


Analysis 4.4. Comparison 4 Circumcision versus waiting list,

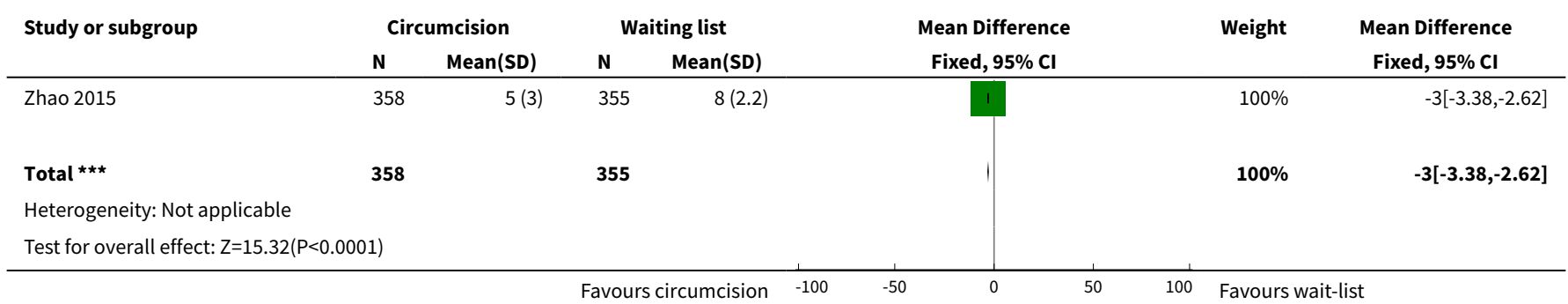

Analysis 4.5. Comparison 4 Circumcision versus waiting list, Outcome 5 Adverse events.

\begin{tabular}{|c|c|c|c|c|c|}
\hline Study or subgroup & $\begin{array}{c}\text { Circumcision } \\
n / N\end{array}$ & $\begin{array}{c}\text { Waiting list } \\
\mathrm{n} / \mathrm{N}\end{array}$ & $\begin{array}{c}\text { Risk Ratio } \\
\text { M-H, Fixed, 95\% Cl }\end{array}$ & Weight & $\begin{array}{c}\text { Risk Ratio } \\
\text { M-H, Fixed, 95\% Cl }\end{array}$ \\
\hline Zhao 2015 & $57 / 358$ & $46 / 355$ & & $100 \%$ & $1.23[0.86,1.76]$ \\
\hline Total $(95 \% \mathrm{Cl})$ & 358 & 355 & & $100 \%$ & $1.23[0.86,1.76]$ \\
\hline \multicolumn{6}{|c|}{ Total events: 57 (Circumcision), 46 (Waiting list) } \\
\hline \multicolumn{6}{|c|}{ Heterogeneity: Not applicable } \\
\hline \multicolumn{6}{|c|}{ Test for overall effect: $\mathrm{Z}=1.12(\mathrm{P}=0.26)$} \\
\hline
\end{tabular}

\section{Comparison 5. Electromagnetic chair versus sham procedure}

\begin{tabular}{|c|c|c|c|c|}
\hline Outcome or subgroup title & No. of studies & $\begin{array}{l}\text { No. of partici- } \\
\text { pants }\end{array}$ & Statistical method & Effect size \\
\hline $\begin{array}{l}1 \text { Prostatitis symptoms (NIH-CPSI } \\
\text { total) }\end{array}$ & 2 & 57 & $\begin{array}{l}\text { Mean Difference (IV, Fixed, 95\% } \\
\mathrm{CI} \text { ) }\end{array}$ & $-3.0[-7.75,1.75]$ \\
\hline $\begin{array}{l}2 \text { Prostatitis symptoms: pain sub- } \\
\text { score }\end{array}$ & 2 & 57 & $\begin{array}{l}\text { Std. Mean Difference (IV, Fixed, } \\
95 \% \mathrm{CI} \text { ) }\end{array}$ & $-0.44[-1.07,0.19]$ \\
\hline $\begin{array}{l}3 \text { Prostatitis symptoms: micturi- } \\
\text { tion subscore }\end{array}$ & 2 & 57 & $\begin{array}{l}\text { Std. Mean Difference (IV, Fixed, } \\
95 \% \mathrm{Cl} \text { ) }\end{array}$ & $0.22[-0.40,0.84]$ \\
\hline $\begin{array}{l}4 \text { Prostatitis symptoms: quality of } \\
\text { life subscore }\end{array}$ & 1 & & $\begin{array}{l}\text { Mean Difference (IV, Fixed, 95\% } \\
\mathrm{CI} \text { ) }\end{array}$ & Totals not selected \\
\hline 5 Adverse events & 2 & 57 & Risk Ratio (M-H, Fixed, 95\% Cl) & $2.18[0.10,46.92]$ \\
\hline 6 Urinary symptoms & 1 & 40 & $\begin{array}{l}\text { Mean Difference (IV, Fixed, 95\% } \\
\mathrm{CI})\end{array}$ & $0.0[-4.13,4.13]$ \\
\hline $\begin{array}{l}7 \text { Prostatitis symptoms (NIH-CPSI } \\
\text { total) - medium term }\end{array}$ & 1 & & $\begin{array}{l}\text { Mean Difference (IV, Fixed, 95\% } \\
\mathrm{CI} \text { ) }\end{array}$ & Totals not selected \\
\hline $\begin{array}{l}8 \text { Prostatitis symptoms: pain sub- } \\
\text { score - medium term }\end{array}$ & 1 & & $\begin{array}{l}\text { Mean Difference (IV, Fixed, 95\% } \\
\mathrm{CI} \text { ) }\end{array}$ & Totals not selected \\
\hline
\end{tabular}




\section{Analysis 5.1. Comparison 5 Electromagnetic chair versus sham} procedure, Outcome 1 Prostatitis symptoms (NIH-CPSI total).

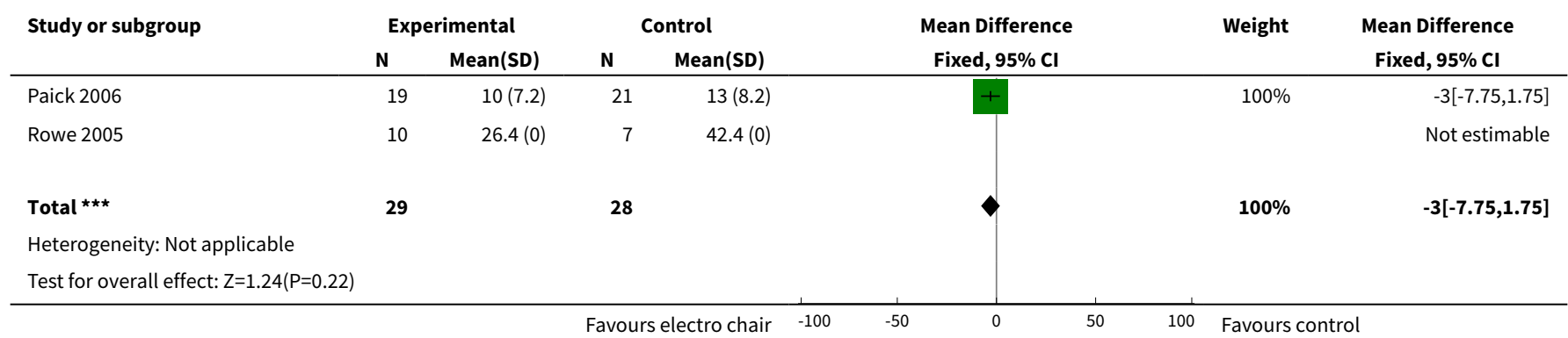

\section{Analysis 5.2. Comparison 5 Electromagnetic chair versus sham} procedure, Outcome 2 Prostatitis symptoms: pain subscore.

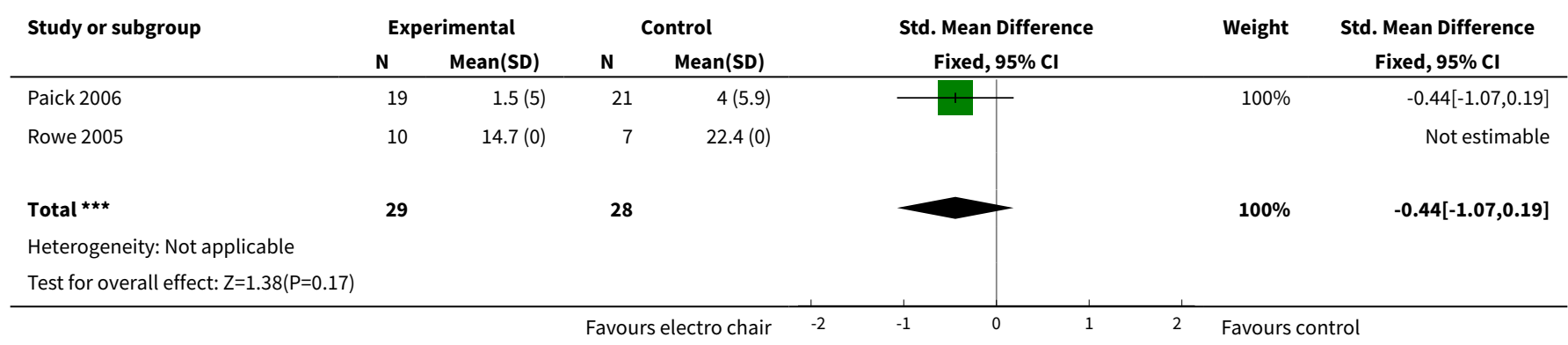

Analysis 5.3. Comparison 5 Electromagnetic chair versus sham procedure, Outcome 3 Prostatitis symptoms: micturition subscore.

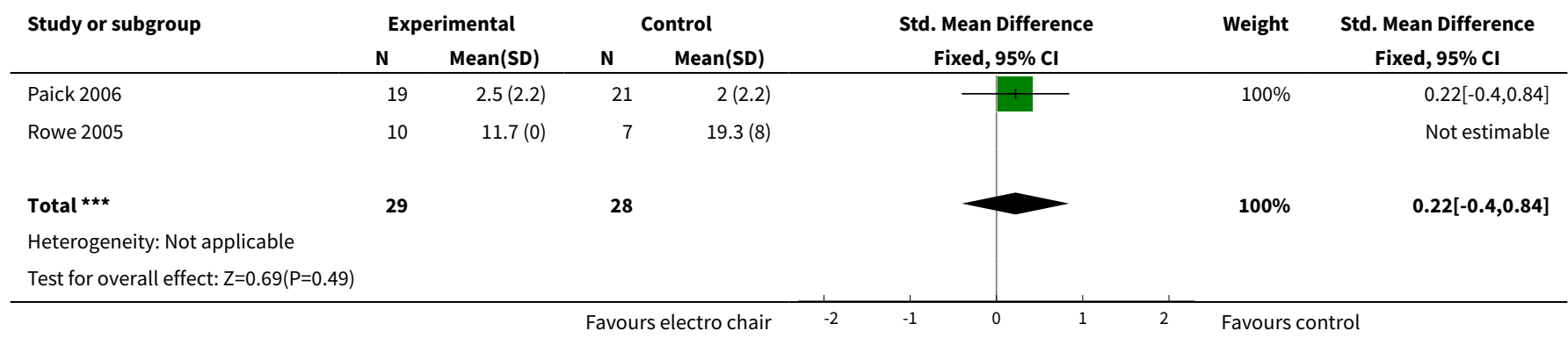

Analysis 5.4. Comparison 5 Electromagnetic chair versus sham procedure, Outcome 4 Prostatitis symptoms: quality of life subscore.

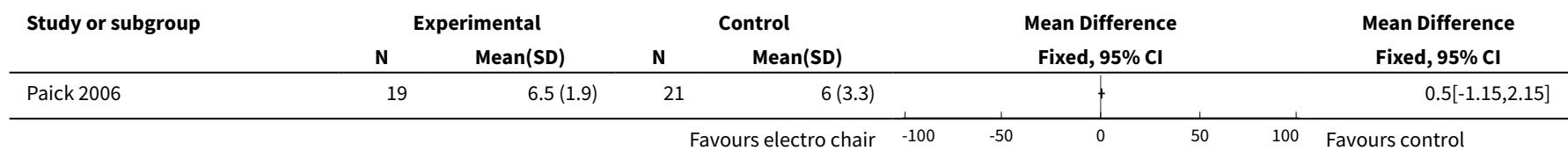


Analysis 5.5. Comparison 5 Electromagnetic chair versus sham procedure, Outcome 5 Adverse events.

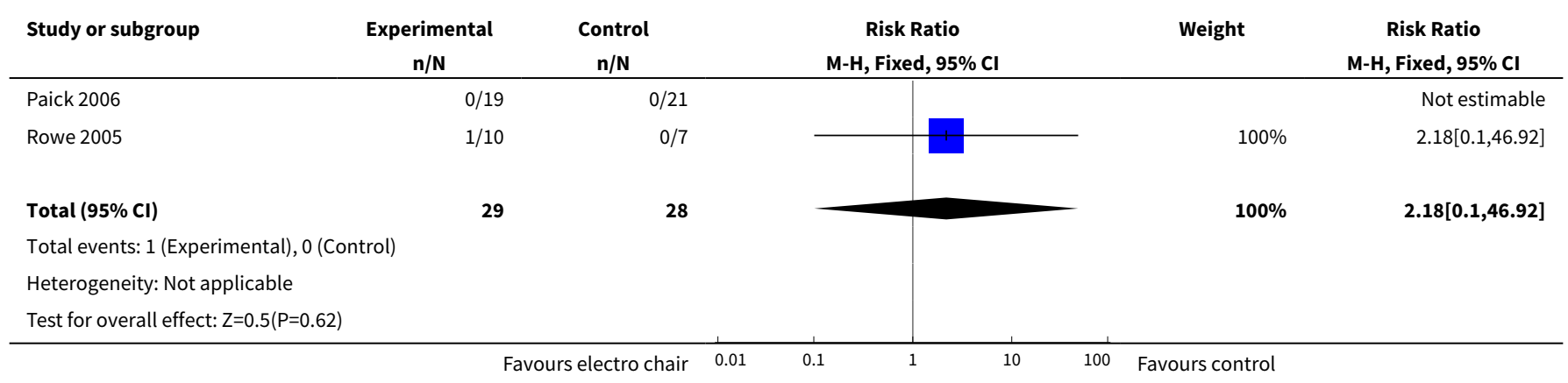

Analysis 5.6. Comparison 5 Electromagnetic chair versus sham procedure, Outcome 6 Urinary symptoms.

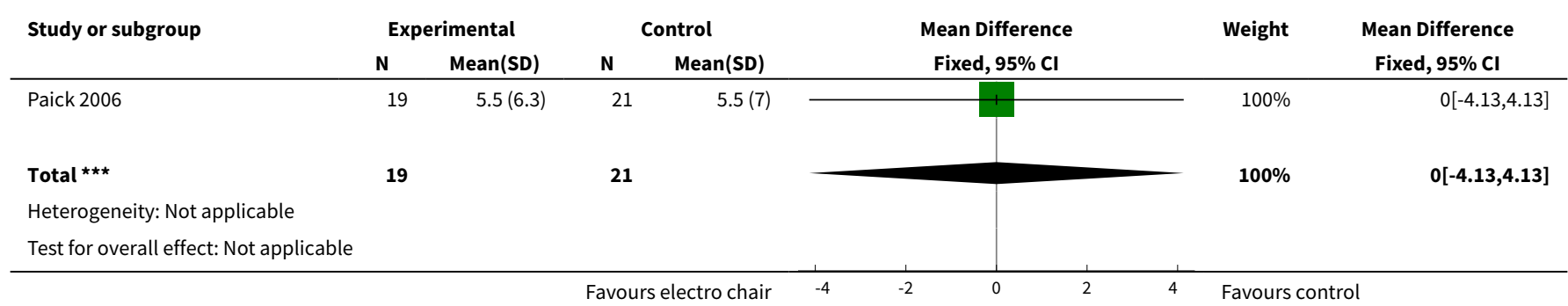

Analysis 5.7. Comparison 5 Electromagnetic chair versus sham procedure, Outcome 7 Prostatitis symptoms (NIH-CPSI total) - medium term.

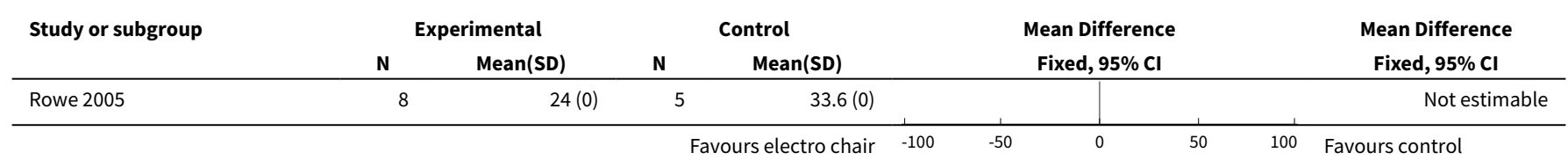

Analysis 5.8. Comparison 5 Electromagnetic chair versus sham procedure, Outcome 8 Prostatitis symptoms: pain subscore - medium term.

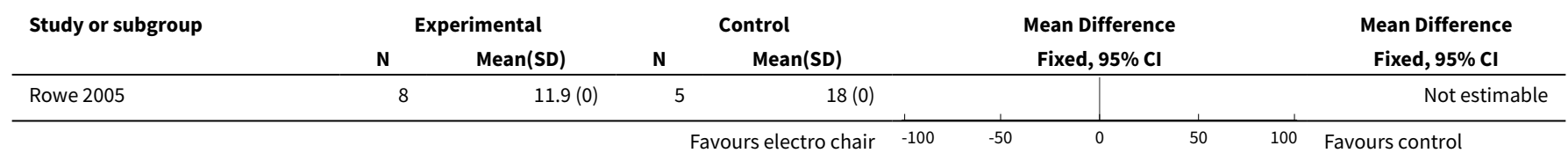


Comparison 6. Lifestyle modifications versus control

\begin{tabular}{lllll}
\hline Outcome or subgroup title & No. of studies & $\begin{array}{l}\text { No. of partici- } \\
\text { pants }\end{array}$ & Statistical method & Effect size \\
\hline 1 Prostatitis symptoms & 1 & 100 & Risk Ratio (M-H, Fixed, 95\% Cl) & $3.9[2.20,6.92]$ \\
\hline
\end{tabular}

Analysis 6.1. Comparison 6 Lifestyle modifications versus control, Outcome 1 Prostatitis symptoms.

\begin{tabular}{|c|c|c|c|c|c|}
\hline Study or subgroup & $\begin{array}{l}\text { Experimental } \\
n / N\end{array}$ & $\begin{array}{c}\text { Control } \\
n / N\end{array}$ & $\begin{array}{c}\text { Risk Ratio } \\
\text { M-H, Fixed, } 95 \% \mathrm{CI}\end{array}$ & Weight & $\begin{array}{c}\text { Risk Ratio } \\
\text { M-H, Fixed, 95\% Cl }\end{array}$ \\
\hline Gallo 2014 & $39 / 50$ & $10 / 50$ & & $100 \%$ & $3.9[2.2,6.92]$ \\
\hline Total $(95 \% \mathrm{Cl})$ & 50 & 50 & & $100 \%$ & $3.9[2.2,6.92]$ \\
\hline \multicolumn{6}{|c|}{ Total events: 39 (Experimental), 10 (Control) } \\
\hline \multicolumn{6}{|c|}{ Heterogeneity: Not applicable } \\
\hline
\end{tabular}

\section{Comparison 7. Physical activity versus control}

\begin{tabular}{lllll}
\hline Outcome or subgroup title & No. of studies & $\begin{array}{l}\text { No. of partici- } \\
\text { pants }\end{array}$ & Statistical method & Effect size \\
\hline 1 Prostatitis symptoms & 1 & 85 & Mean Difference (IV, Fixed, 95\% Cl) & $-2.50[-4.69,-0.31]$ \\
\hline $\begin{array}{l}\text { 2 Prostatitis symptoms: pain } \\
\text { subscore }\end{array}$ & 1 & Mean Difference (IV, Fixed, 95\% Cl) & Totals not selected \\
\hline $\begin{array}{l}\text { 3 Prostatitis symptoms: mic- } \\
\text { turition subscore }\end{array}$ & 1 & Mean Difference (IV, Fixed, 95\% Cl) & Totals not selected \\
\hline $\begin{array}{l}\text { 4 Prostatitis symptoms: qual- } \\
\text { ity of life subscore }\end{array}$ & 1 & Mean Difference (IV, Fixed, 95\% Cl) & Totals not selected \\
\hline $\begin{array}{l}\text { 5 Anxiety } \\
\text { 6 Depression }\end{array}$ & 1 & Mean Difference (IV, Fixed, 95\% Cl) & Totals not selected \\
\hline
\end{tabular}

Analysis 7.1. Comparison 7 Physical activity versus control, Outcome 1 Prostatitis symptoms.

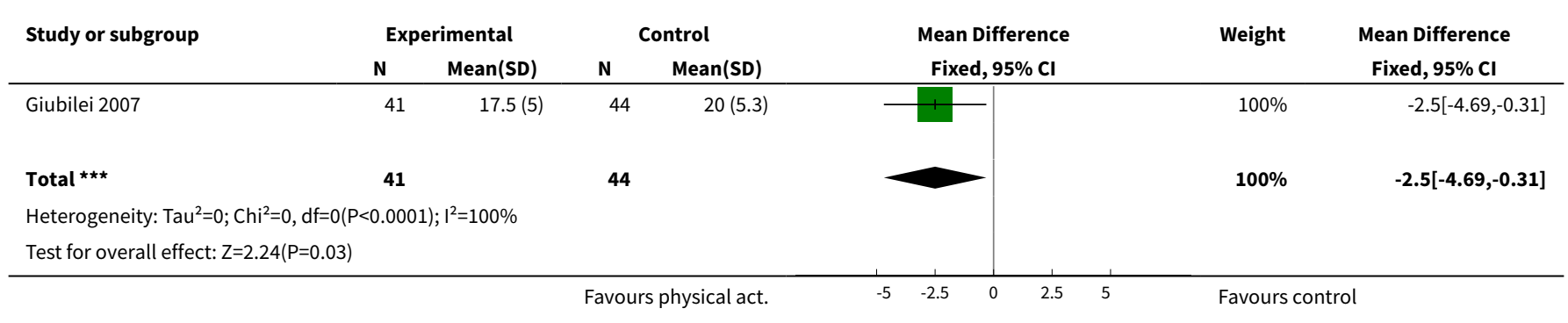


Analysis 7.2. Comparison 7 Physical activity versus control, Outcome 2 Prostatitis symptoms: pain subscore.

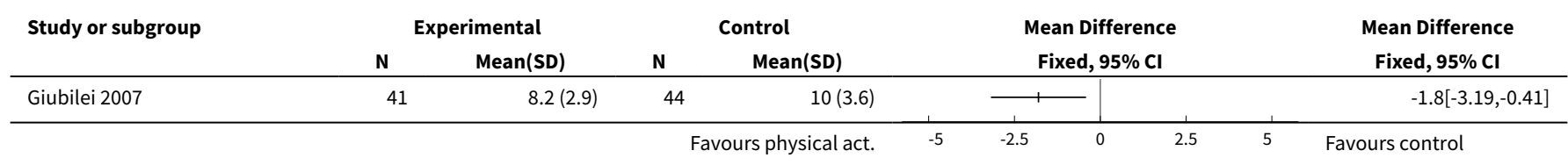

Analysis 7.3. Comparison 7 Physical activity versus control, Outcome 3 Prostatitis symptoms: micturition subscore.

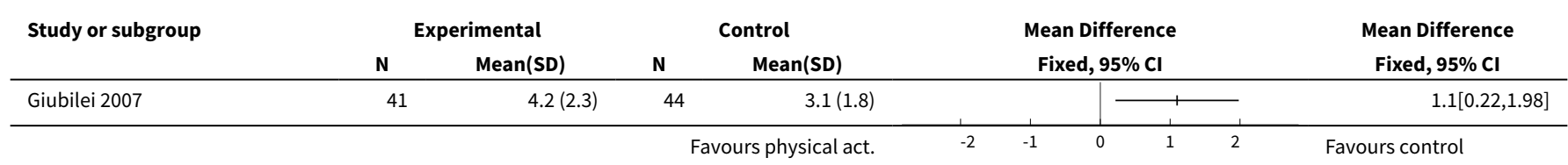

Analysis 7.4. Comparison 7 Physical activity versus control,

Outcome 4 Prostatitis symptoms: quality of life subscore.

\begin{tabular}{lcccccc|c} 
Study or subgroup & \multicolumn{2}{c}{$\begin{array}{c}\text { Experimental } \\
\text { Mean(SD) }\end{array}$} & N & $\begin{array}{c}\text { Control } \\
\text { Mean(SD) }\end{array}$ & \multicolumn{2}{c}{$\begin{array}{c}\text { Mean Difference } \\
\text { Fixed, 95\% Cl }\end{array}$} \\
\hline Giubilei 2007 & 41 & $5.1(2.1)$ & 44 & $6.9(2.1)$ & & Fixed, 95\% Cl & $-1.8[-2.69,-0.91]$ \\
\hline
\end{tabular}

Analysis 7.5. Comparison 7 Physical activity versus control, Outcome 5 Anxiety.

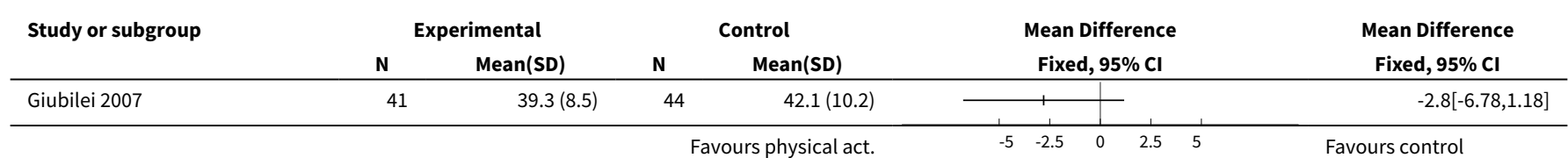

Analysis 7.6. Comparison 7 Physical activity versus control, Outcome 6 Depression.

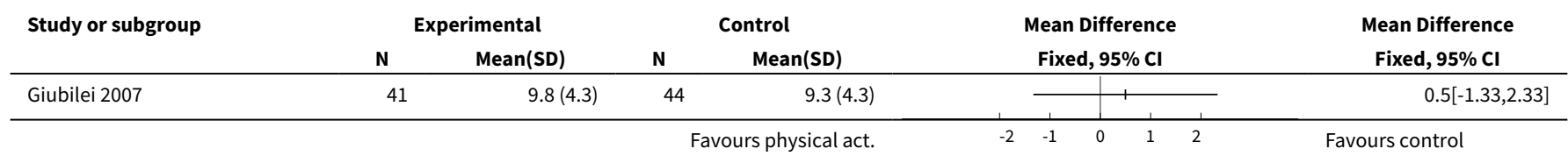


Comparison 8. Prostatic massage versus control

\begin{tabular}{llll}
\hline Outcome or subgroup title & No. of studies & $\begin{array}{l}\text { No. of partici- } \\
\text { pants }\end{array}$ & Statistical method \\
\hline $\begin{array}{llll}\text { 1 Prostatitis symptoms } & 4 & \text { Effect size } & \text { Mean Difference (IV, Fixed, 95\% } \\
\text { Cl) }\end{array}$ & $1.10[-5.63,3.43]$ & Mean Difference (IV, Fixed, 95\% & Totals not selected \\
\hline $\begin{array}{l}\text { 2 Prostatitis symptoms: pain sub- } \\
\text { score }\end{array}$ & 2 & Cl) \\
\hline $\begin{array}{l}3 \text { Prostatitis symptoms: micturi- } \\
\text { tion subscore }\end{array}$ & 2 & Mean Difference (IV, Fixed, 95\% \\
\hline $\begin{array}{l}\text { 4 Prostatitis symptoms: quality of } \\
\text { life subscore }\end{array}$ & 2 & Totals not selected \\
\hline
\end{tabular}

Analysis 8.1. Comparison 8 Prostatic massage versus control, Outcome 1 Prostatitis symptoms.

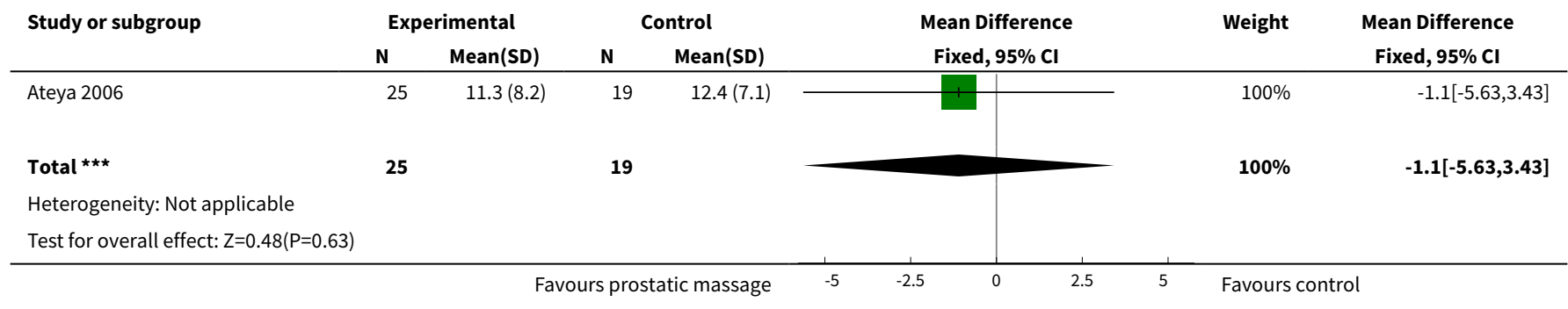

\section{Analysis 8.2. Comparison 8 Prostatic massage versus control, Outcome 2 Prostatitis symptoms: pain subscore.}

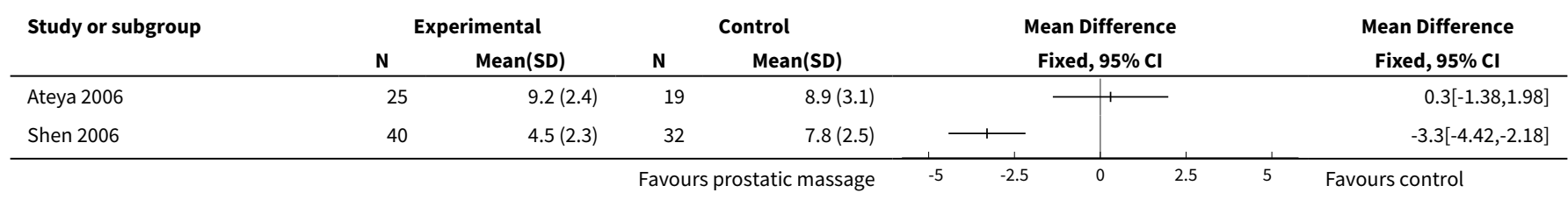

Analysis 8.3. Comparison 8 Prostatic massage versus control, Outcome 3 Prostatitis symptoms: micturition subscore.

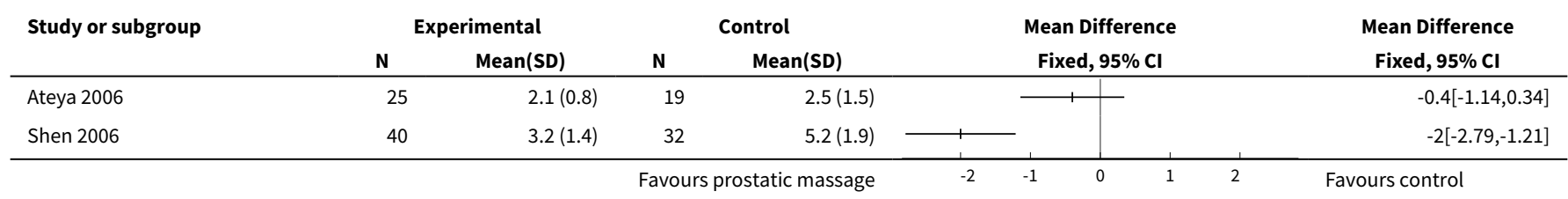


Analysis 8.4. Comparison 8 Prostatic massage versus control, Outcome 4 Prostatitis symptoms: quality of life subscore.

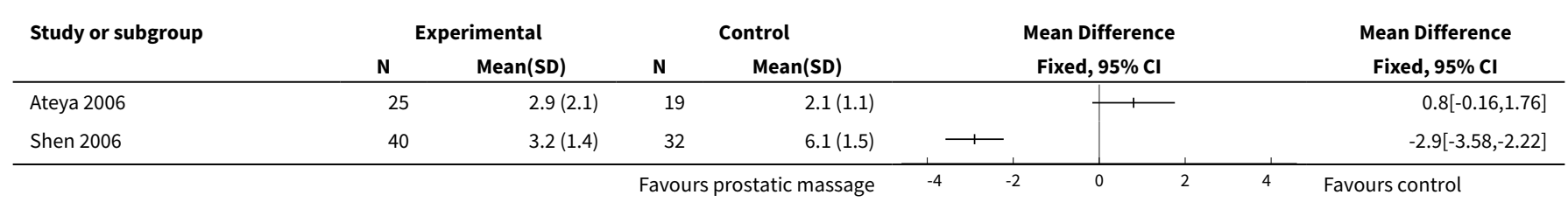

\section{Comparison 9. Extracorporeal shockwave therapy (ESWT) versus control}

\begin{tabular}{|c|c|c|c|c|}
\hline Outcome or subgroup title & No. of studies & $\begin{array}{l}\text { No. of partici- } \\
\text { pants }\end{array}$ & Statistical method & Effect size \\
\hline $\begin{array}{l}1 \text { Prostatitis symptoms }(\mathrm{NIH}- \\
\text { CPSI total) }\end{array}$ & 3 & 157 & $\begin{array}{l}\text { Mean Difference (IV, Random, } \\
95 \% \mathrm{CI} \text { ) }\end{array}$ & $-6.18[-7.46,-4.89]$ \\
\hline $\begin{array}{l}\text { 1.1 Compared to sham proce- } \\
\text { dure }\end{array}$ & 2 & 97 & $\begin{array}{l}\text { Mean Difference (IV, Random, } \\
95 \% \mathrm{CI})\end{array}$ & $-6.14[-7.87,-4.41]$ \\
\hline $\begin{array}{l}1.2 \text { Compared to no interven- } \\
\text { tion }\end{array}$ & 1 & 60 & $\begin{array}{l}\text { Mean Difference (IV, Random, } \\
95 \% \mathrm{CI})\end{array}$ & $-6.64[-10.17,-3.11]$ \\
\hline $\begin{array}{l}2 \text { Prostatitis symptoms: pain } \\
\text { subscore }\end{array}$ & 2 & 97 & $\begin{array}{l}\text { Mean Difference (IV, Random, } \\
95 \% \mathrm{CI} \text { ) }\end{array}$ & $-4.64[-5.38,-3.89]$ \\
\hline $\begin{array}{l}2.1 \text { Compared to sham proce- } \\
\text { dure }\end{array}$ & 1 & 37 & $\begin{array}{l}\text { Mean Difference (IV, Random, } \\
95 \% \mathrm{CI})\end{array}$ & $-4.74[-5.54,-3.94]$ \\
\hline $\begin{array}{l}2.2 \text { Compared to no interven- } \\
\text { tion }\end{array}$ & 1 & 60 & $\begin{array}{l}\text { Mean Difference (IV, Random, } \\
95 \% \mathrm{CI} \text { ) }\end{array}$ & $-3.83[-6.03,-1.63]$ \\
\hline $\begin{array}{l}3 \text { Prostatitis symptoms: micturi- } \\
\text { tion subscore }\end{array}$ & 2 & 97 & $\begin{array}{l}\text { Mean Difference (IV, Fixed, 95\% } \\
\mathrm{CI} \text { ) }\end{array}$ & $-0.53[1.00,-0.07]$ \\
\hline $\begin{array}{l}\text { 3.1 Compared to sham proce- } \\
\text { dure }\end{array}$ & 1 & 37 & $\begin{array}{l}\text { Mean Difference (IV, Fixed, 95\% } \\
\mathrm{CI})\end{array}$ & $-1.79[-2.59,-0.99]$ \\
\hline $\begin{array}{l}3.2 \text { Compared to no interven- } \\
\text { tion }\end{array}$ & 1 & 60 & $\begin{array}{l}\text { Mean Difference (IV, Fixed, 95\% } \\
\mathrm{CI})\end{array}$ & $0.10[-0.47,0.67]$ \\
\hline $\begin{array}{l}4 \text { Prostatitis symptoms: quality } \\
\text { of life subscore }\end{array}$ & 2 & 97 & $\begin{array}{l}\text { Mean Difference (IV, Random, } \\
95 \% \mathrm{CI})\end{array}$ & $-1.84[-2.41,-1.27]$ \\
\hline $\begin{array}{l}\text { 4.1 Compared to sham proce- } \\
\text { dure }\end{array}$ & 1 & 37 & $\begin{array}{l}\text { Mean Difference (IV, Random, } \\
95 \% \mathrm{CI})\end{array}$ & $-1.73[-2.35,-1.11]$ \\
\hline $\begin{array}{l}4.2 \text { Compared to no interven- } \\
\text { tion }\end{array}$ & 1 & 60 & $\begin{array}{l}\text { Mean Difference (IV, Random, } \\
95 \% \mathrm{CI})\end{array}$ & $-2.46[-3.94,-0.98]$ \\
\hline 5 Prostatitis symptoms & 2 & 135 & $\begin{array}{l}\text { Risk Ratio (M-H, Random, 95\% } \\
\text { Cl) }\end{array}$ & $6.20[0.48,79.79]$ \\
\hline $\begin{array}{l}6 \text { Prostatitis symptoms (total } \\
\text { score) - long term }\end{array}$ & 2 & 97 & $\begin{array}{l}\text { Mean Difference (IV, Random, } \\
95 \% \mathrm{CI} \text { ) }\end{array}$ & $-2.23[-5.98,1.53]$ \\
\hline
\end{tabular}




\begin{tabular}{|c|c|c|c|c|}
\hline Outcome or subgroup title & No. of studies & $\begin{array}{l}\text { No. of partici- } \\
\text { pants }\end{array}$ & Statistical method & Effect size \\
\hline $\begin{array}{l}\text { 6.1 Compared to sham proce- } \\
\text { dure }\end{array}$ & 1 & 37 & $\begin{array}{l}\text { Mean Difference (IV, Random, } \\
95 \% \mathrm{CI})\end{array}$ & $-0.59[-1.42,0.24]$ \\
\hline $\begin{array}{l}6.2 \text { Compared to no interven- } \\
\text { tion }\end{array}$ & 1 & 60 & $\begin{array}{l}\text { Mean Difference (IV, Random, } \\
95 \% \mathrm{CI} \text { ) }\end{array}$ & $-4.47[-7.60,-1.34]$ \\
\hline $\begin{array}{l}7 \text { Prostatitis symptoms: pain } \\
\text { subscore - long term }\end{array}$ & 2 & 97 & $\begin{array}{l}\text { Mean Difference (IV, Random, } \\
95 \% \mathrm{CI} \text { ) }\end{array}$ & $-2.08[-6.25,2.09]$ \\
\hline $\begin{array}{l}7.1 \text { Compared to sham proce- } \\
\text { dure }\end{array}$ & 1 & 37 & $\begin{array}{l}\text { Mean Difference (IV, Random, } \\
95 \% \mathrm{CI})\end{array}$ & $-0.01[-1.26,1.24]$ \\
\hline $\begin{array}{l}7.2 \text { Compared to no interven- } \\
\text { tion }\end{array}$ & 1 & 60 & $\begin{array}{l}\text { Mean Difference (IV, Random, } \\
95 \% \mathrm{CI} \text { ) }\end{array}$ & $-4.27[-6.15,-2.39]$ \\
\hline $\begin{array}{l}8 \text { Prostatitis symptoms: micturi- } \\
\text { tion subscore - long term }\end{array}$ & 2 & 97 & $\begin{array}{l}\text { Mean Difference (IV, Random, } \\
95 \% \mathrm{CI} \text { ) }\end{array}$ & $-0.64[-1.19,-0.10]$ \\
\hline $\begin{array}{l}\text { 8.1 Compared to sham proce- } \\
\text { dure }\end{array}$ & 1 & 37 & $\begin{array}{l}\text { Mean Difference (IV, Random, } \\
95 \% \mathrm{CI})\end{array}$ & $-0.35[-1.50,0.80]$ \\
\hline $\begin{array}{l}8.2 \text { Compared to no interven- } \\
\text { tion }\end{array}$ & 1 & 60 & $\begin{array}{l}\text { Mean Difference (IV, Random, } \\
95 \% \mathrm{CI} \text { ) }\end{array}$ & $-0.73[-1.35,-0.11]$ \\
\hline $\begin{array}{l}9 \text { Prostatitis symptoms: quality } \\
\text { of life subscore - long term }\end{array}$ & 2 & 97 & $\begin{array}{l}\text { Mean Difference (IV, Random, } \\
95 \% \mathrm{CI} \text { ) }\end{array}$ & $-0.97[-2.78,0.85]$ \\
\hline $\begin{array}{l}\text { 9.1 Compared to sham proce- } \\
\text { dure }\end{array}$ & 1 & 37 & $\begin{array}{l}\text { Mean Difference (IV, Random, } \\
95 \% \mathrm{CI})\end{array}$ & $-0.16[-0.98,0.66]$ \\
\hline $\begin{array}{l}9.2 \text { Compared to no interven- } \\
\text { tion }\end{array}$ & 1 & 60 & $\begin{array}{l}\text { Mean Difference (IV, Random, } \\
95 \% \mathrm{CI} \text { ) }\end{array}$ & $-2.03[-3.62,-0.44]$ \\
\hline 10 Adverse events & 3 & 195 & Risk Ratio (M-H, Fixed, 95\% Cl) & $1.22[0.59,2.51]$ \\
\hline 11 Sexual dysfunction & 1 & 60 & $\begin{array}{l}\text { Mean Difference (IV, Fixed, 95\% } \\
\mathrm{CI})\end{array}$ & $3.34[2.68,4.00]$ \\
\hline 12 Urinary symptoms & 1 & 60 & $\begin{array}{l}\text { Mean Difference (IV, Fixed, 95\% } \\
\text { CI) }\end{array}$ & $-4.50[-5.14,-3.86]$ \\
\hline
\end{tabular}

\section{Analysis 9.1. Comparison 9 Extracorporeal shockwave therapy (ESWT) versus control, Outcome 1 Prostatitis symptoms (NIH-CPSI total).}

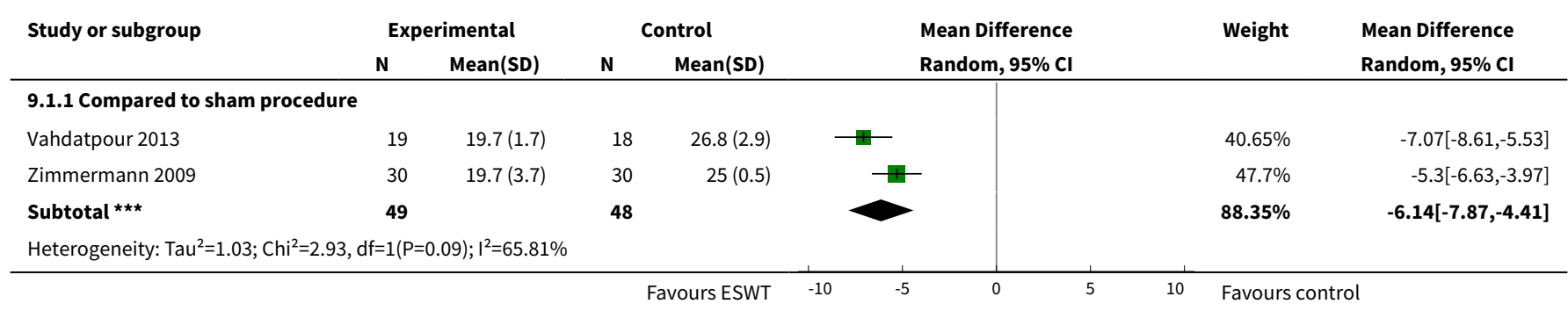




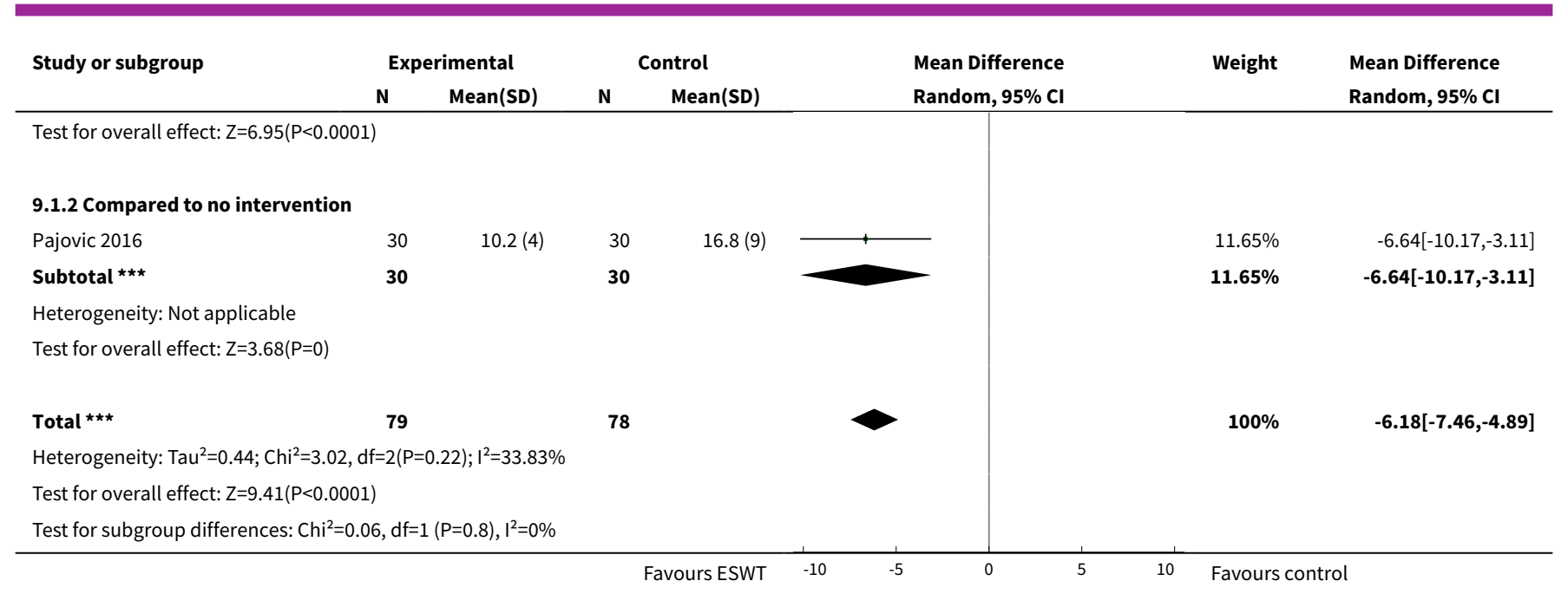

\section{Analysis 9.2. Comparison 9 Extracorporeal shockwave therapy (ESWT)} versus control, Outcome 2 Prostatitis symptoms: pain subscore.

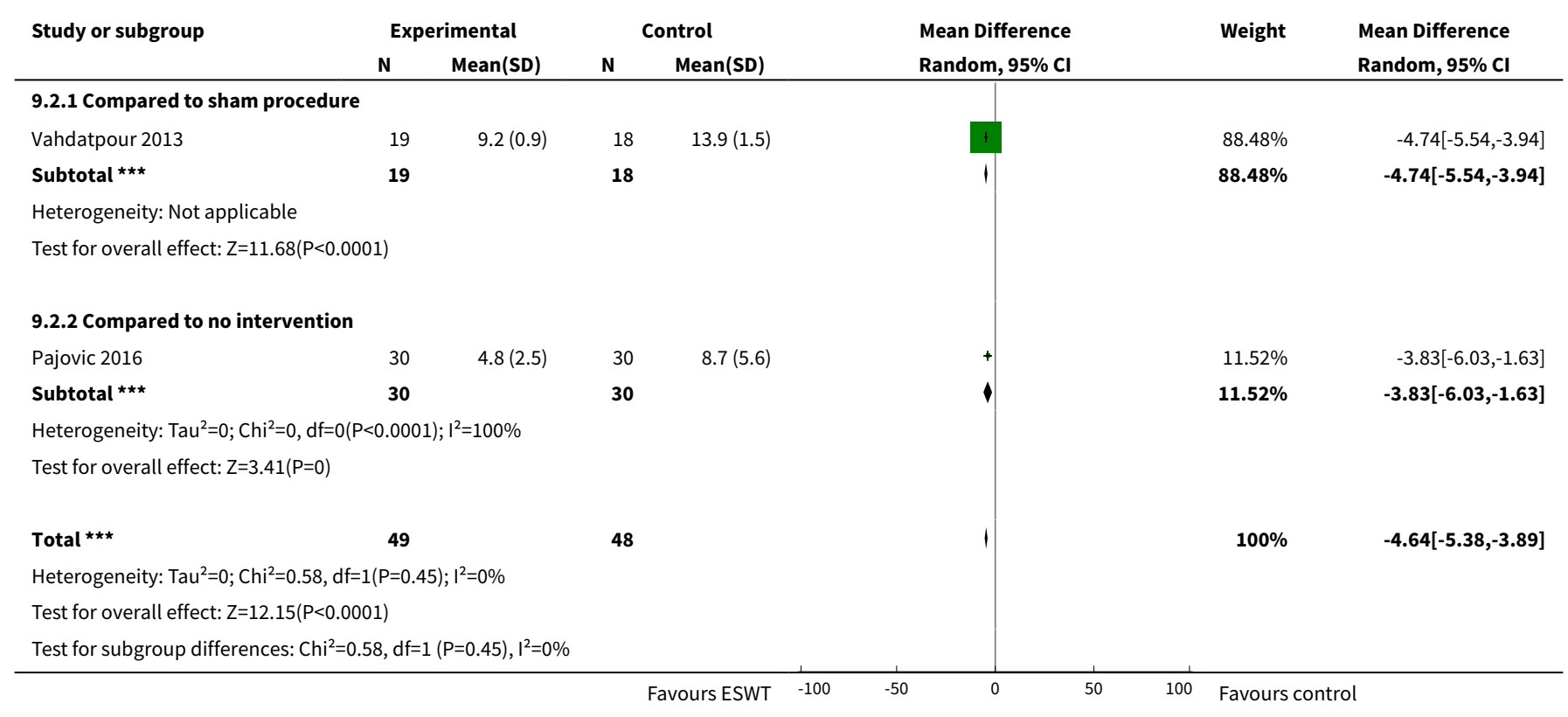

Analysis 9.3. Comparison 9 Extracorporeal shockwave therapy (ESWT) versus control, Outcome 3 Prostatitis symptoms: micturition subscore.

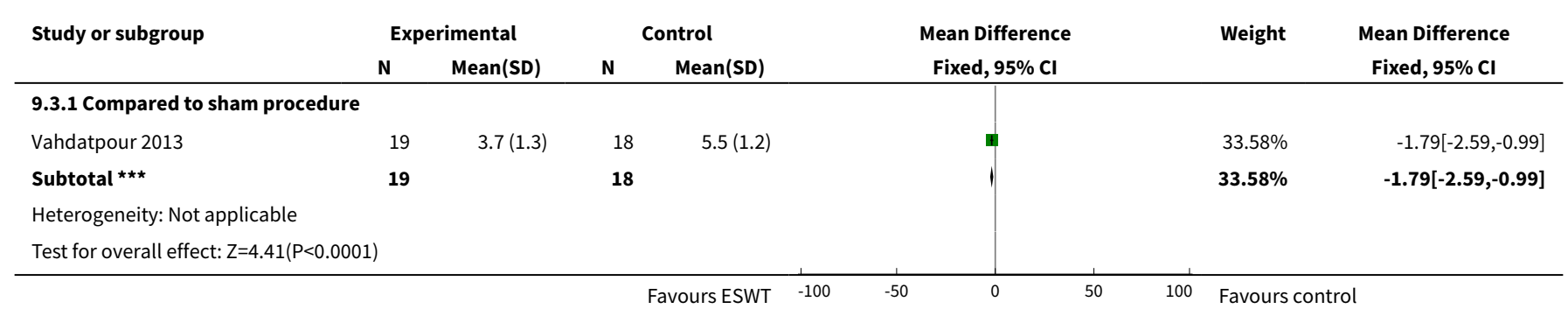




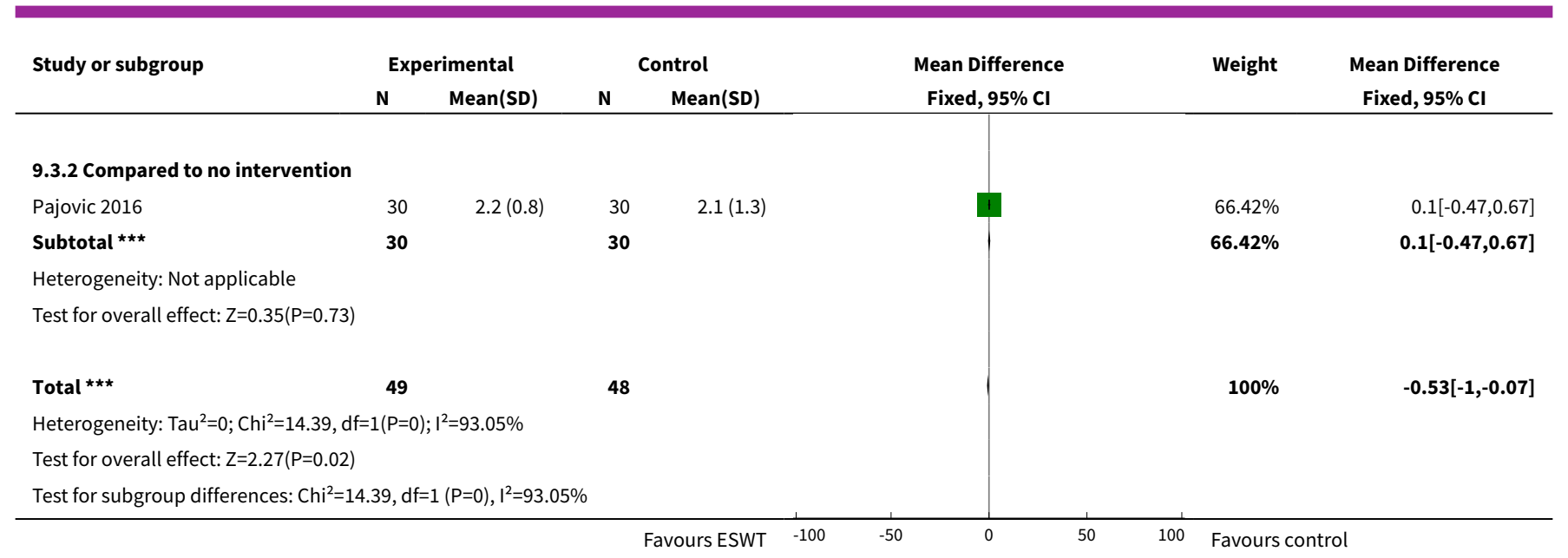

Analysis 9.4. Comparison 9 Extracorporeal shockwave therapy (ESWT) versus control, Outcome 4 Prostatitis symptoms: quality of life subscore.

\begin{tabular}{|c|c|c|c|c|c|c|c|}
\hline \multirow[t]{2}{*}{ Study or subgroup } & \multicolumn{2}{|c|}{ Experimental } & \multicolumn{2}{|c|}{ Control } & \multirow{2}{*}{$\begin{array}{l}\text { Mean Difference } \\
\text { Random, } 95 \% \mathrm{Cl}\end{array}$} & \multirow[t]{2}{*}{ Weight } & \multirow{2}{*}{$\begin{array}{l}\text { Mean Difference } \\
\text { Random, } 95 \% \mathrm{Cl}\end{array}$} \\
\hline & $\mathbf{N}$ & $\operatorname{Mean}(S D)$ & $\mathbf{N}$ & Mean(SD) & & & \\
\hline \multicolumn{8}{|c|}{ 9.4.1 Compared to sham procedure } \\
\hline Vahdatpour 2013 & 19 & $6.1(0.7)$ & 18 & $7.8(1.2)$ & & $85 \%$ & $-1.73[-2.35,-1.11]$ \\
\hline Subtotal $\star \star \star ~$ & 19 & & 18 & & & $85 \%$ & $-1.73[-2.35,-1.11]$ \\
\hline \multicolumn{8}{|c|}{ Heterogeneity: Not applicable } \\
\hline \multicolumn{8}{|c|}{ Test for overall effect: $Z=5.45(P<0.0001)$} \\
\hline \multicolumn{8}{|c|}{ 9.4.2 Compared to no intervention } \\
\hline Pajovic 2016 & 30 & $2.9(1.6)$ & 30 & $5.4(3.8)$ & & $15 \%$ & $-2.46[-3.94,-0.98]$ \\
\hline Subtotal $\star \star \star$ & 30 & & 30 & & & $15 \%$ & $-2.46[-3.94,-0.98]$ \\
\hline \multicolumn{8}{|c|}{ Heterogeneity: Not applicable } \\
\hline \multicolumn{8}{|c|}{ Test for overall effect: $Z=3.26(P=0)$} \\
\hline \multicolumn{8}{|c|}{ Heterogeneity: $\mathrm{Tau}^{2}=0 ; \mathrm{Chi}^{2}=0.79, \mathrm{df}=1(\mathrm{P}=0.37) ;\left.\right|^{2}=0 \%$} \\
\hline \multicolumn{8}{|c|}{ Test for overall effect: $Z=6.29(P<0.0001)$} \\
\hline Test for subgroup dif & $9, \mathrm{df}=$ & $P=0.37), I^{2}=09$ & & & & & \\
\hline
\end{tabular}

Analysis 9.5. Comparison 9 Extracorporeal shockwave therapy (ESWT) versus control, Outcome 5 Prostatitis symptoms.

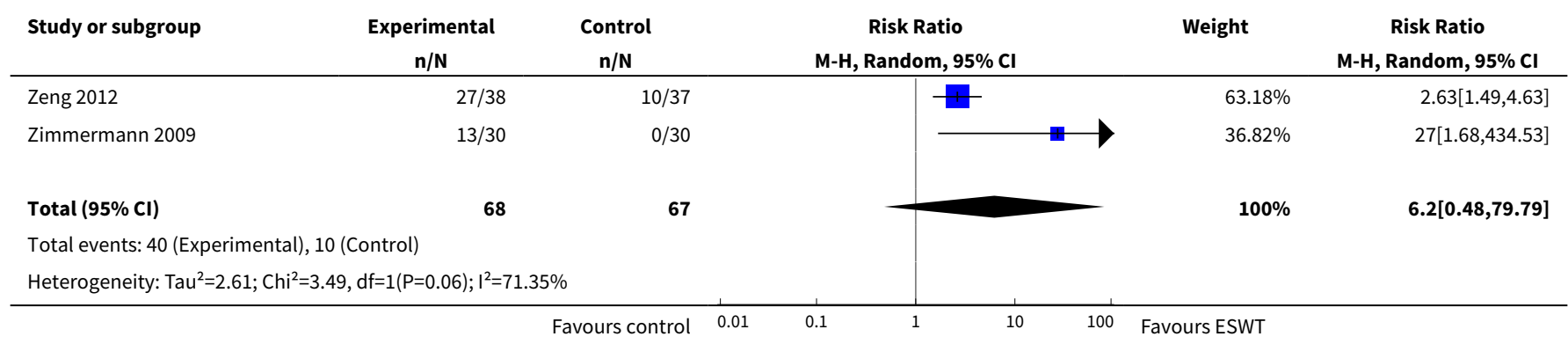




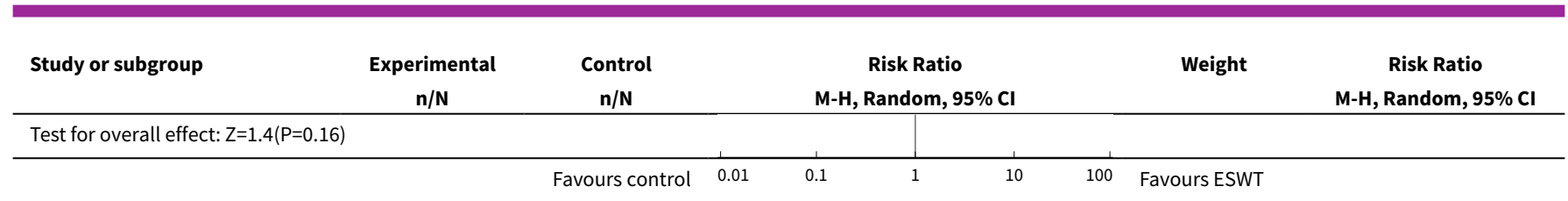

Analysis 9.6. Comparison 9 Extracorporeal shockwave therapy (ESWT) versus control, Outcome 6 Prostatitis symptoms (total score) - long term.

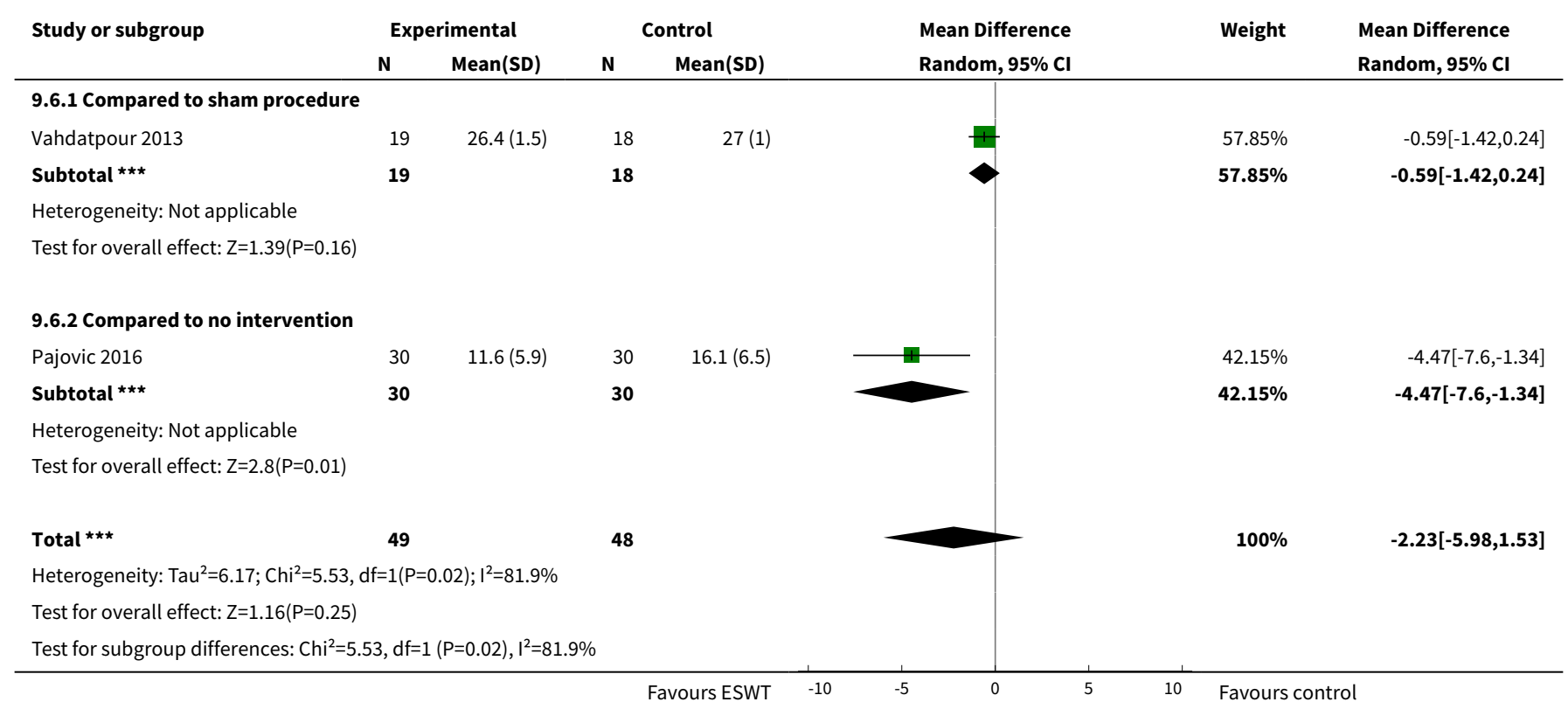

Analysis 9.7. Comparison 9 Extracorporeal shockwave therapy (ESWT) versus control, Outcome 7 Prostatitis symptoms: pain subscore - long term.

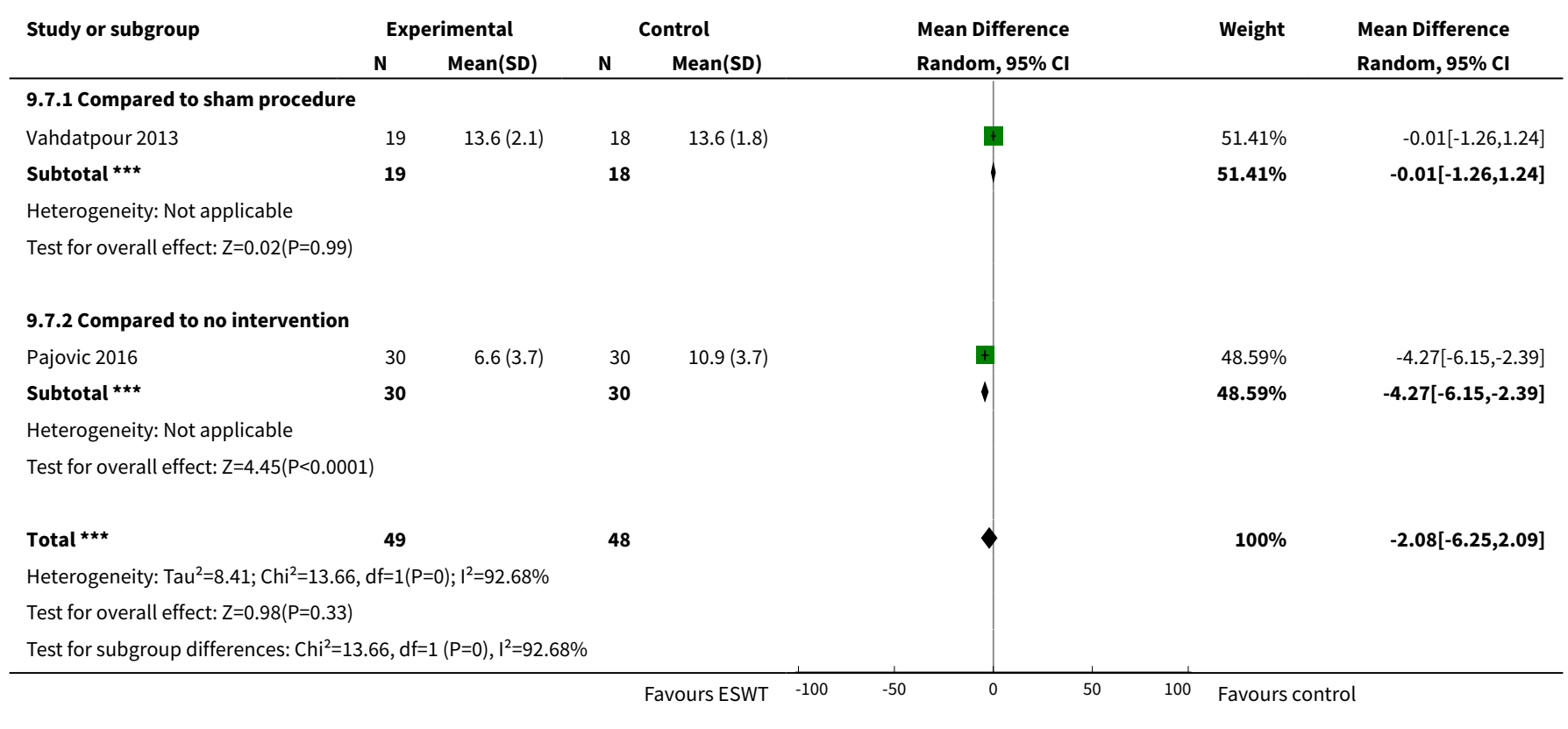


Analysis 9.8. Comparison 9 Extracorporeal shockwave therapy (ESWT) versus control, Outcome 8 Prostatitis symptoms: micturition subscore - long term.

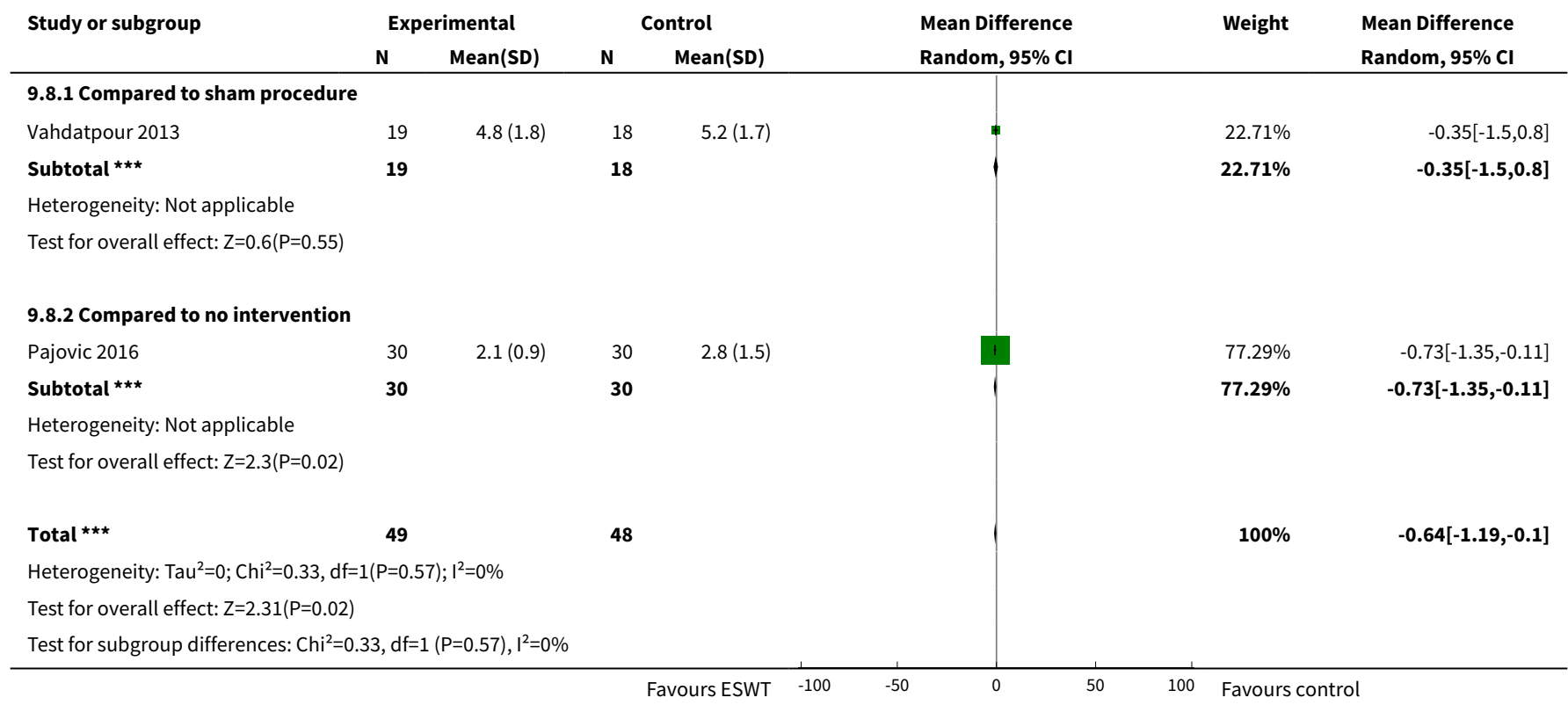

Analysis 9.9. Comparison 9 Extracorporeal shockwave therapy (ESWT) versus control, Outcome 9 Prostatitis symptoms: quality of life subscore - long term.

\begin{tabular}{|c|c|c|c|c|c|c|c|}
\hline \multirow[t]{2}{*}{ Study or subgroup } & \multicolumn{2}{|c|}{ Experimental } & \multicolumn{2}{|c|}{ Control } & \multirow{2}{*}{$\begin{array}{l}\text { Mean Difference } \\
\text { Random, } 95 \% \mathrm{Cl}\end{array}$} & \multirow[t]{2}{*}{ Weight } & \multirow{2}{*}{$\begin{array}{l}\text { Mean Difference } \\
\text { Random, } 95 \% \mathrm{Cl}\end{array}$} \\
\hline & $\mathbf{N}$ & Mean(SD) & $\mathbf{N}$ & Mean(SD) & & & \\
\hline \multicolumn{8}{|c|}{ 9.9.1 Compared to sham procedure } \\
\hline Vahdatpour 2013 & 19 & $8(1.2)$ & 18 & $8.2(1.4)$ & & $56.92 \%$ & $-0.16[-0.98,0.66]$ \\
\hline Subtotal $* \star \star$ & 19 & & 18 & & & $56.92 \%$ & $-0.16[-0.98,0.66]$ \\
\hline \multicolumn{8}{|c|}{ Heterogeneity: Not applicable } \\
\hline \multicolumn{8}{|c|}{ Test for overall effect: $\mathrm{Z}=0.38(\mathrm{P}=0.7)$} \\
\hline \multicolumn{8}{|c|}{ 9.9.2 Compared to no intervention } \\
\hline Pajovic 2016 & 30 & $4(2.6)$ & 30 & $6.1(3.6)$ & & $43.08 \%$ & $-2.03[-3.62,-0.44]$ \\
\hline Subtotal ${ }^{\star \star \star}$ & 30 & & 30 & & & $43.08 \%$ & $-2.03[-3.62,-0.44]$ \\
\hline \multicolumn{8}{|c|}{ Heterogeneity: Not applicable } \\
\hline \multicolumn{8}{|c|}{ Test for overall effect: $Z=2.5(P=0.01)$} \\
\hline \multicolumn{8}{|c|}{ Heterogeneity: $\mathrm{Tau}^{2}=1.33 ; \mathrm{Chi}^{2}=4.2, \mathrm{df}=1(\mathrm{P}=0.04) ; \mathrm{I}^{2}=76.18 \%$} \\
\hline \multicolumn{8}{|c|}{ Test for overall effect: $\mathrm{Z}=1.04(\mathrm{P}=0.3)$} \\
\hline Test for subgroup dif & $\mathrm{df}=1$ & $=0.04), I^{2}=76$. & & & & & \\
\hline
\end{tabular}


Analysis 9.10. Comparison 9 Extracorporeal shockwave therapy (ESWT) versus control, Outcome 10 Adverse events.

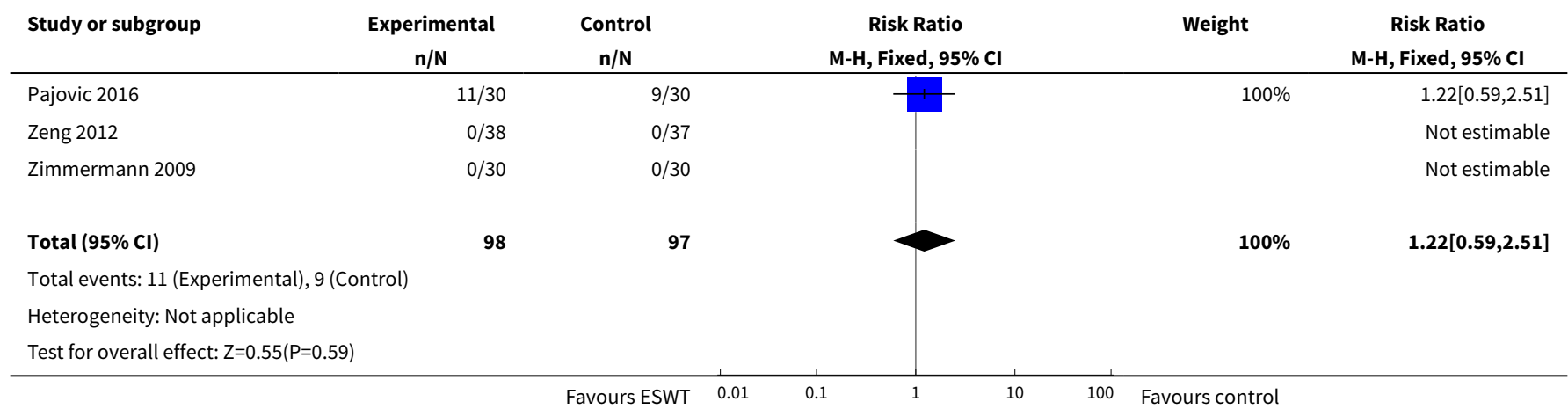

Analysis 9.11. Comparison 9 Extracorporeal shockwave therapy (ESWT) versus control, Outcome 11 Sexual dysfunction.

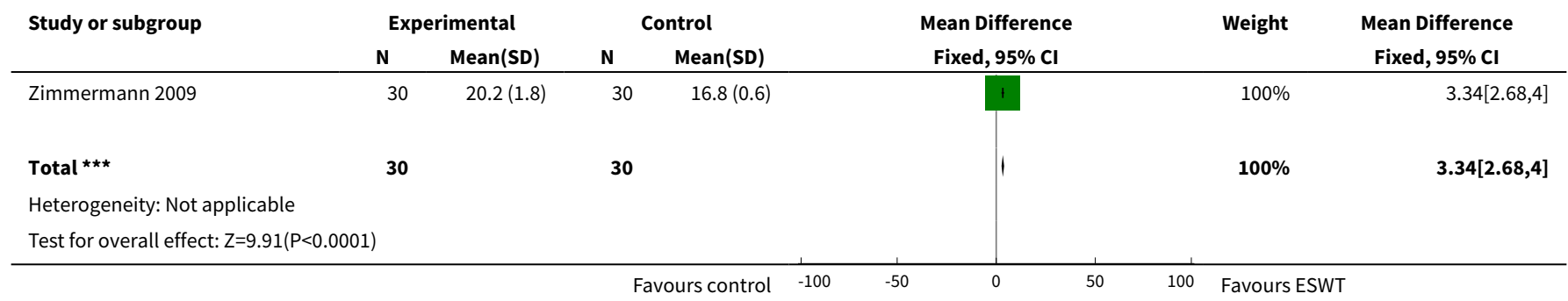

Analysis 9.12. Comparison 9 Extracorporeal shockwave therapy (ESWT) versus control, Outcome 12 Urinary symptoms.

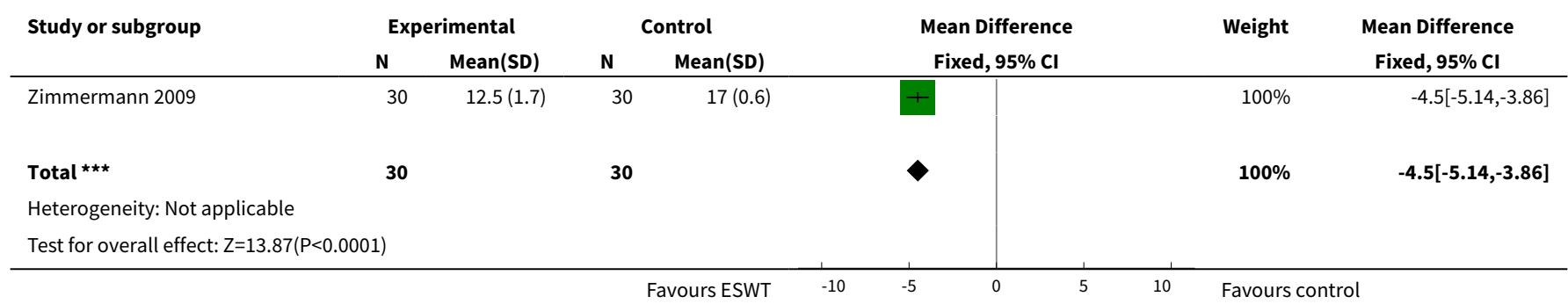

\section{Comparison 10. Transrectal thermotherapy (TRT) versus medical treatment}

\begin{tabular}{|c|c|c|c|c|}
\hline Outcome or subgroup title & No. of studies & $\begin{array}{l}\text { No. of partici- } \\
\text { pants }\end{array}$ & Statistical method & Effect size \\
\hline $\begin{array}{l}1 \text { Prostatitis symptoms (NIH-CPSI to- } \\
\text { tal) }\end{array}$ & 2 & & $\begin{array}{l}\text { Mean Difference (IV, Fixed, 95\% } \\
\mathrm{CI})\end{array}$ & Subtotals only \\
\hline 1.1 Compared to medical therapy & 2 & 140 & $\begin{array}{l}\text { Mean Difference (IV, Fixed, 95\% } \\
\mathrm{Cl} \text { ) }\end{array}$ & $\begin{array}{l}-2.50[-3.82 \\
-1.18]\end{array}$ \\
\hline
\end{tabular}




\begin{tabular}{|c|c|c|c|c|}
\hline Outcome or subgroup title & No. of studies & $\begin{array}{l}\text { No. of partici- } \\
\text { pants }\end{array}$ & Statistical method & Effect size \\
\hline $\begin{array}{l}1.2 \text { Add-on to medical therapy vs } \\
\text { medical therapy alone }\end{array}$ & 2 & 145 & $\begin{array}{l}\text { Mean Difference (IV, Fixed, 95\% } \\
\mathrm{CI})\end{array}$ & $\begin{array}{l}-4.34[-5.65 \\
-3.04]\end{array}$ \\
\hline $\begin{array}{l}2 \text { Prostatitis symptoms: pain sub- } \\
\text { score }\end{array}$ & 1 & & $\begin{array}{l}\text { Mean Difference (IV, Fixed, 95\% } \\
\mathrm{CI} \text { ) }\end{array}$ & $\begin{array}{l}\text { Totals not select- } \\
\text { ed }\end{array}$ \\
\hline 2.1 Compared to medical therapy & 1 & & $\begin{array}{l}\text { Mean Difference (IV, Fixed, 95\% } \\
\mathrm{CI} \text { ) }\end{array}$ & $0.0[0.0,0.0]$ \\
\hline $\begin{array}{l}2.2 \text { Add-on to medical therapy vs } \\
\text { medical therapy alone }\end{array}$ & 1 & & $\begin{array}{l}\text { Mean Difference (IV, Fixed, 95\% } \\
\mathrm{Cl} \text { ) }\end{array}$ & $0.0[0.0,0.0]$ \\
\hline $\begin{array}{l}3 \text { Prostatitis symptoms: micturition } \\
\text { subscore }\end{array}$ & 1 & & $\begin{array}{l}\text { Mean Difference (IV, Fixed, 95\% } \\
\mathrm{Cl} \text { ) }\end{array}$ & $\begin{array}{l}\text { Totals not select- } \\
\text { ed }\end{array}$ \\
\hline 3.1 Compared to medical therapy & 1 & & $\begin{array}{l}\text { Mean Difference (IV, Fixed, 95\% } \\
\mathrm{CI} \text { ) }\end{array}$ & $0.0[0.0,0.0]$ \\
\hline $\begin{array}{l}\text { 3.2 Add-on to medical therapy vs } \\
\text { medical therapy alone }\end{array}$ & 1 & & $\begin{array}{l}\text { Mean Difference (IV, Fixed, 95\% } \\
\mathrm{CI} \text { ) }\end{array}$ & $0.0[0.0,0.0]$ \\
\hline $\begin{array}{l}4 \text { Prostatitis symptoms: quality of life } \\
\text { subscore }\end{array}$ & 1 & & $\begin{array}{l}\text { Mean Difference (IV, Fixed, 95\% } \\
\mathrm{CI} \text { ) }\end{array}$ & $\begin{array}{l}\text { Totals not select- } \\
\text { ed }\end{array}$ \\
\hline 4.1 Compared to medical therapy & 1 & & $\begin{array}{l}\text { Mean Difference (IV, Fixed, 95\% } \\
\mathrm{Cl} \text { ) }\end{array}$ & $0.0[0.0,0.0]$ \\
\hline $\begin{array}{l}4.2 \text { Add-on to medical therapy vs } \\
\text { medical therapy alone }\end{array}$ & 1 & & $\begin{array}{l}\text { Mean Difference (IV, Fixed, 95\% } \\
\mathrm{Cl} \text { ) }\end{array}$ & $0.0[0.0,0.0]$ \\
\hline
\end{tabular}

Analysis 10.1. Comparison 10 Transrectal thermotherapy (TRT) versus medical treatment, Outcome 1 Prostatitis symptoms (NIH-CPSI total).

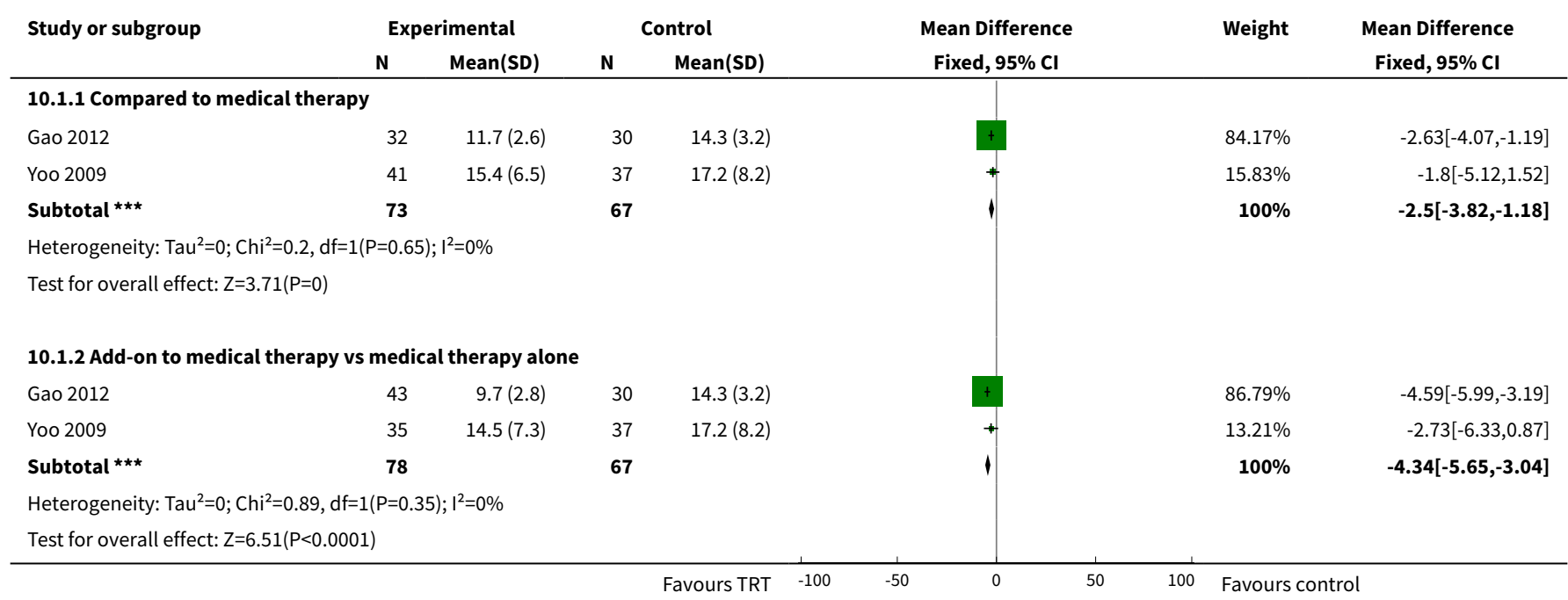


Analysis 10.2. Comparison 10 Transrectal thermotherapy (TRT) versus medical treatment, Outcome 2 Prostatitis symptoms: pain subscore.

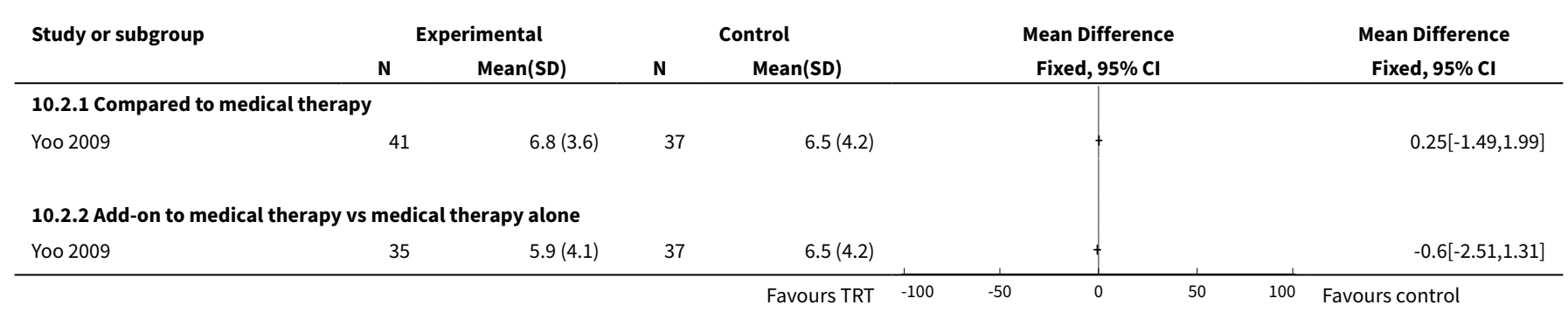

Analysis 10.3. Comparison 10 Transrectal thermotherapy (TRT) versus medical treatment, Outcome 3 Prostatitis symptoms: micturition subscore.

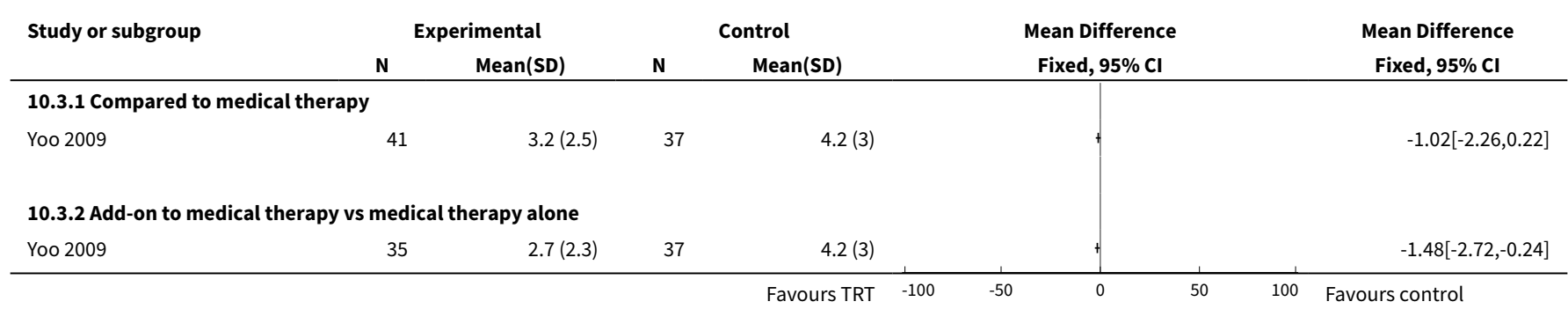

Analysis 10.4. Comparison 10 Transrectal thermotherapy (TRT) versus medical treatment, Outcome 4 Prostatitis symptoms: quality of life subscore.

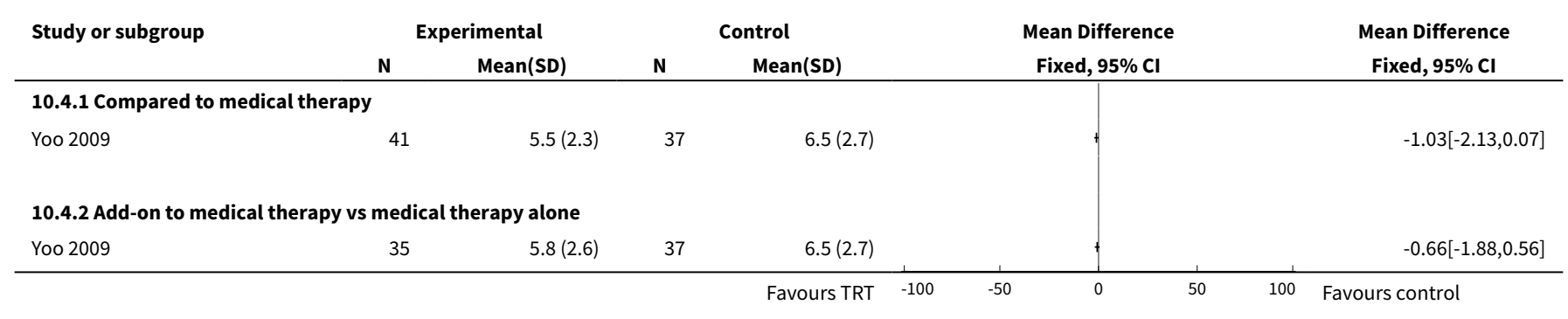

Comparison 11. Biofeedback with or without electrical stimulation versus usual care

\begin{tabular}{lllll}
\hline Outcome or subgroup title & No. of studies & $\begin{array}{l}\text { No. of partici- } \\
\text { pants }\end{array}$ & Statistical method & Effect size \\
\hline $\begin{array}{l}1 \text { Prostatitis symptoms (NIH-CPSI to- } \\
\text { tal) }\end{array}$ & 1 & $\begin{array}{l}\text { Mean Difference (IV, Fixed, 95\% } \\
\text { Cl) }\end{array}$ & $\begin{array}{l}\text { Totals not select- } \\
\text { ed }\end{array}$ \\
\hline $\begin{array}{l}1.1 \text { Biofeedback + electrical stimula- } \\
\text { tion vs usual care }\end{array}$ & 1 & $\begin{array}{l}\text { Mean Difference (IV, Fixed, 95\% } \\
\text { CI) }\end{array}$ & $0.0[0.0,0.0]$ \\
\hline
\end{tabular}




\begin{tabular}{|c|c|c|c|c|}
\hline Outcome or subgroup title & No. of studies & $\begin{array}{l}\text { No. of partici- } \\
\text { pants }\end{array}$ & Statistical method & Effect size \\
\hline 1.2 Biofeedback vs usual care & 1 & & $\begin{array}{l}\text { Mean Difference (IV, Fixed, 95\% } \\
\mathrm{CI} \text { ) }\end{array}$ & $0.0[0.0,0.0]$ \\
\hline $\begin{array}{l}1.3 \text { Electrical stimulation vs usual } \\
\text { care }\end{array}$ & 1 & & $\begin{array}{l}\text { Mean Difference (IV, Fixed, 95\% } \\
\mathrm{CI})\end{array}$ & $0.0[0.0,0.0]$ \\
\hline $\begin{array}{l}2 \text { Prostatitis symptoms: pain sub- } \\
\text { score }\end{array}$ & 1 & & $\begin{array}{l}\text { Mean Difference (IV, Fixed, 95\% } \\
\mathrm{CI} \text { ) }\end{array}$ & $\begin{array}{l}\text { Totals not select- } \\
\text { ed }\end{array}$ \\
\hline $\begin{array}{l}2.1 \text { Biofeedback + electrical stimula- } \\
\text { tion vs usual care }\end{array}$ & 1 & & $\begin{array}{l}\text { Mean Difference (IV, Fixed, 95\% } \\
\mathrm{CI} \text { ) }\end{array}$ & $0.0[0.0,0.0]$ \\
\hline 2.2 Biofeedback vs usual care & 1 & & $\begin{array}{l}\text { Mean Difference (IV, Fixed, 95\% } \\
\mathrm{CI} \text { ) }\end{array}$ & $0.0[0.0,0.0]$ \\
\hline $\begin{array}{l}2.3 \text { Electrical stimulation vs usual } \\
\text { care }\end{array}$ & 1 & & $\begin{array}{l}\text { Mean Difference (IV, Fixed, 95\% } \\
\mathrm{CI} \text { ) }\end{array}$ & $0.0[0.0,0.0]$ \\
\hline $\begin{array}{l}3 \text { Prostatitis symptoms: micturition } \\
\text { subscore }\end{array}$ & 1 & & $\begin{array}{l}\text { Mean Difference (IV, Fixed, 95\% } \\
\mathrm{CI} \text { ) }\end{array}$ & $\begin{array}{l}\text { Totals not select- } \\
\text { ed }\end{array}$ \\
\hline $\begin{array}{l}\text { 3.1 Biofeedback + electrical Stimula- } \\
\text { tion vs usual care }\end{array}$ & 1 & & $\begin{array}{l}\text { Mean Difference (IV, Fixed, 95\% } \\
\mathrm{CI} \text { ) }\end{array}$ & $0.0[0.0,0.0]$ \\
\hline 3.2 Biofeedback vs usual care & 1 & & $\begin{array}{l}\text { Mean Difference (IV, Fixed, 95\% } \\
\mathrm{CI})\end{array}$ & $0.0[0.0,0.0]$ \\
\hline $\begin{array}{l}3.3 \text { Electrical stimulation vs usual } \\
\text { care }\end{array}$ & 1 & & $\begin{array}{l}\text { Mean Difference (IV, Fixed, 95\% } \\
\mathrm{CI})\end{array}$ & $0.0[0.0,0.0]$ \\
\hline $\begin{array}{l}4 \text { Prostatitis symptoms: quality of life } \\
\text { subscore }\end{array}$ & 1 & & $\begin{array}{l}\text { Mean Difference (IV, Fixed, 95\% } \\
\mathrm{CI})\end{array}$ & $\begin{array}{l}\text { Totals not select- } \\
\text { ed }\end{array}$ \\
\hline $\begin{array}{l}4.1 \text { Biofeedback + electrical stimula- } \\
\text { tion vs usual care }\end{array}$ & 1 & & $\begin{array}{l}\text { Mean Difference (IV, Fixed, 95\% } \\
\mathrm{CI})\end{array}$ & $0.0[0.0,0.0]$ \\
\hline 4.2 Biofeedback vs usual care & 1 & & $\begin{array}{l}\text { Mean Difference (IV, Fixed, 95\% } \\
\mathrm{CI} \text { ) }\end{array}$ & $0.0[0.0,0.0]$ \\
\hline $\begin{array}{l}4.3 \text { Electrical stimulation vs usual } \\
\text { care }\end{array}$ & 1 & & $\begin{array}{l}\text { Mean Difference (IV, Fixed, 95\% } \\
\mathrm{CI} \text { ) }\end{array}$ & $0.0[0.0,0.0]$ \\
\hline
\end{tabular}

Analysis 11.1. Comparison 11 Biofeedback with or without electrical stimulation versus usual care, Outcome 1 Prostatitis symptoms (NIH-CPSI total).

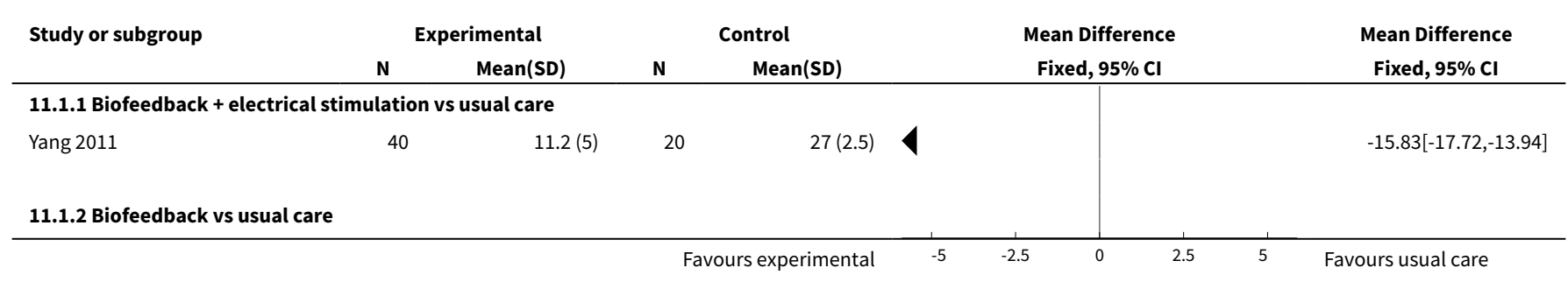




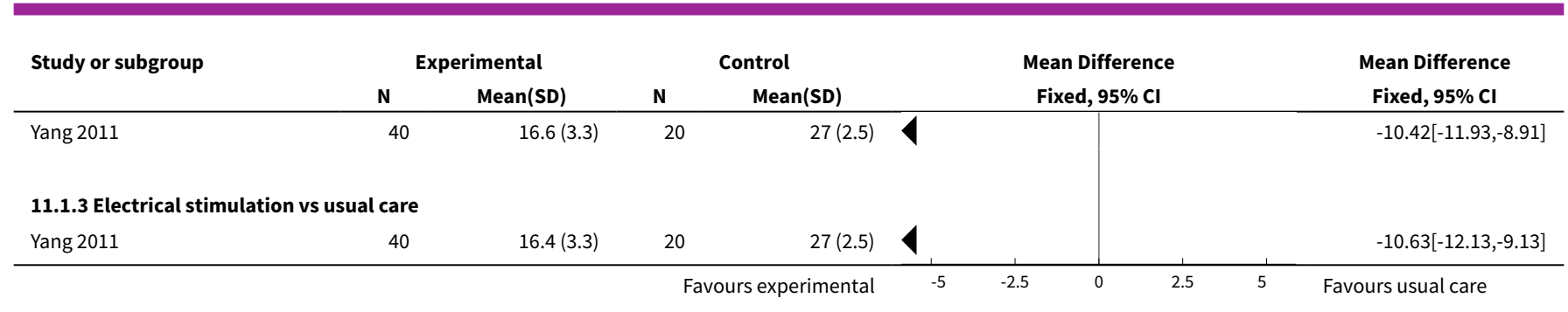

Analysis 11.2. Comparison 11 Biofeedback with or without electrical stimulation versus usual care, Outcome 2 Prostatitis symptoms: pain subscore.

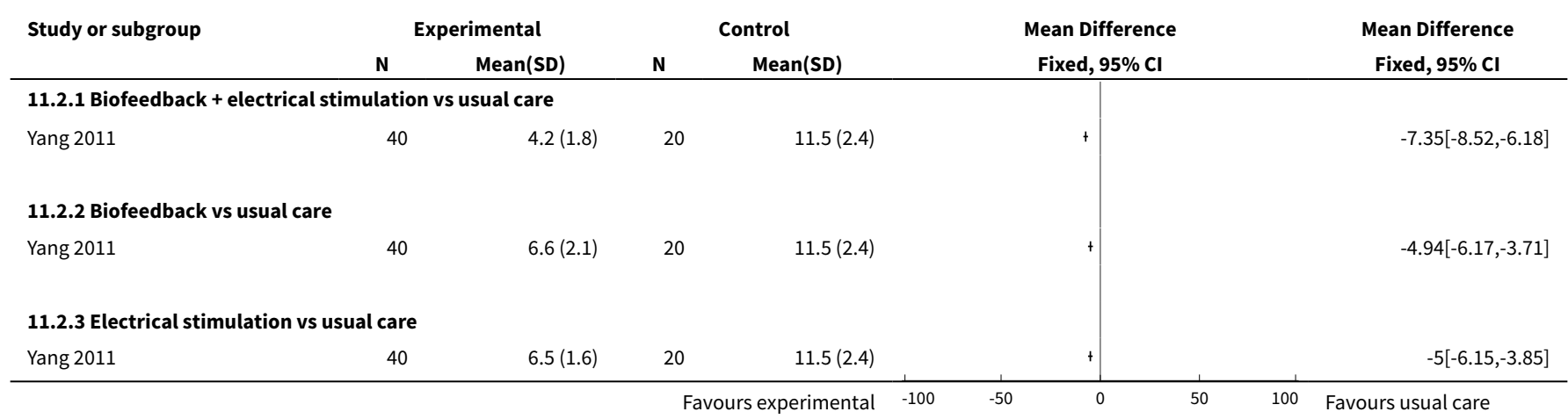

Analysis 11.3. Comparison 11 Biofeedback with or without electrical stimulation versus usual care, Outcome 3 Prostatitis symptoms: micturition subscore.

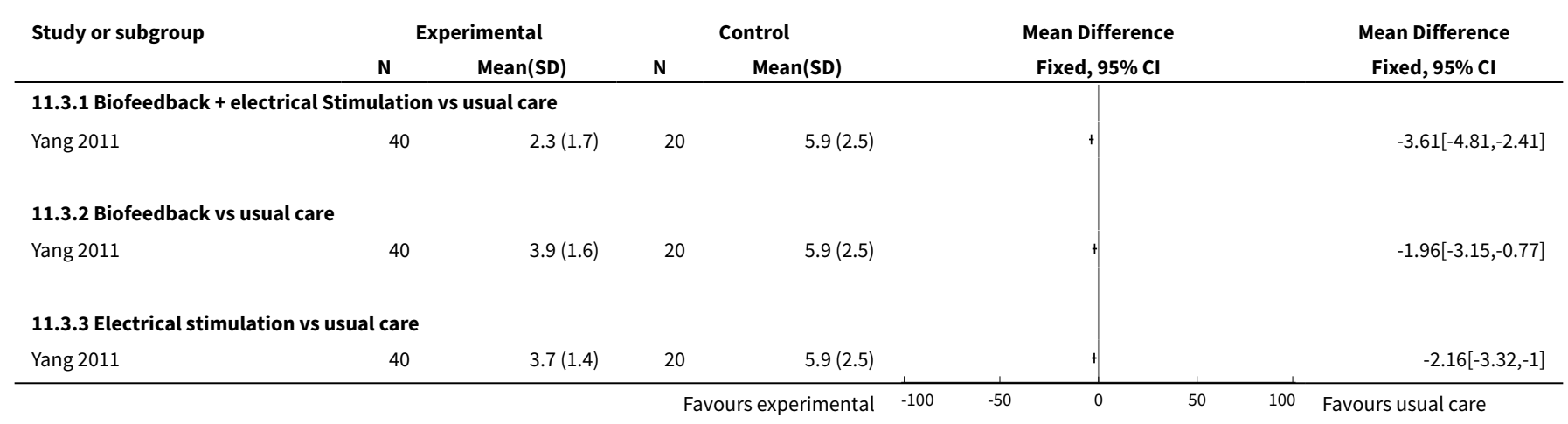

Analysis 11.4. Comparison 11 Biofeedback with or without electrical stimulation versus usual care, Outcome 4 Prostatitis symptoms: quality of life subscore.

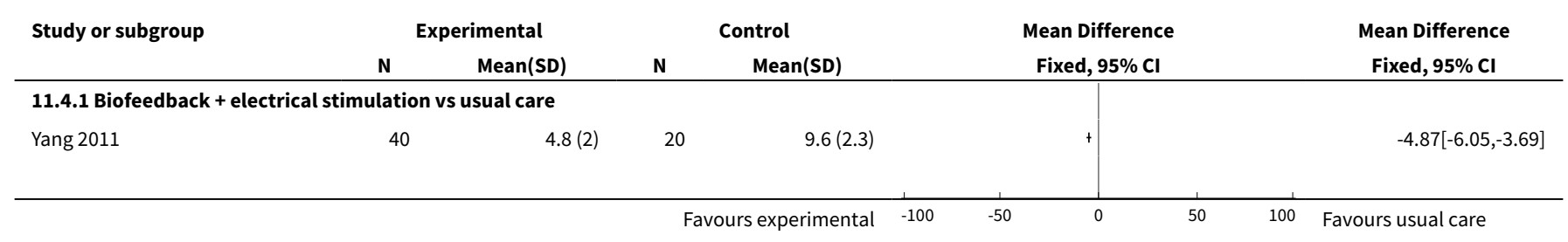




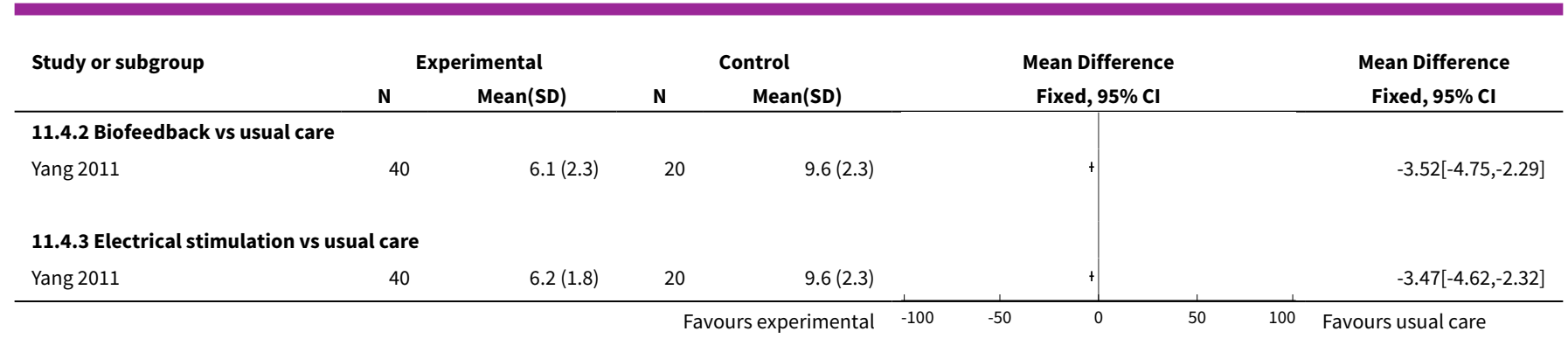

\section{Comparison 12. External radiofrequency hyperthermia with or without terazosin}

\begin{tabular}{lllll}
\hline $\begin{array}{l}\text { Outcome or subgroup ti- } \\
\text { tle }\end{array}$ & No. of studies & $\begin{array}{l}\text { No. of partici- } \\
\text { pants }\end{array}$ & Statistical method & Effect size \\
\hline 1 Prostatitis symptoms & 1 & Mean Difference (IV, Fixed, 95\% Cl) & Totals not selected \\
\hline 2 Adverse events & 1 & Risk Ratio (M-H, Fixed, 95\% Cl) & Totals not selected \\
\hline
\end{tabular}

\section{Analysis 12.1. Comparison 12 External radiofrequency hyperthermia with or without terazosin, Outcome 1 Prostatitis symptoms.}

\begin{tabular}{|c|c|c|c|c|c|c|c|}
\hline \multirow[t]{2}{*}{ Study or subgroup } & \multicolumn{2}{|c|}{ Experimental } & \multicolumn{2}{|c|}{ Control } & \multirow{2}{*}{\multicolumn{2}{|c|}{$\begin{array}{c}\text { Mean Difference } \\
\text { Fixed, } 95 \% \mathrm{Cl}\end{array}$}} & \multirow{2}{*}{$\begin{array}{c}\text { Mean Difference } \\
\text { Fixed, } 95 \% \mathrm{Cl}\end{array}$} \\
\hline & $\mathbf{N}$ & Mean(SD) & $\mathbf{N}$ & Mean(SD) & & & \\
\hline Wang 2002 & 74 & $2.1(1.4)$ & 60 & $4.1(1.4)$ & $\longrightarrow$ & & $-2[-2.48,-1.52]$ \\
\hline
\end{tabular}

Analysis 12.2. Comparison 12 External radiofrequency hyperthermia with or without terazosin, Outcome 2 Adverse events.

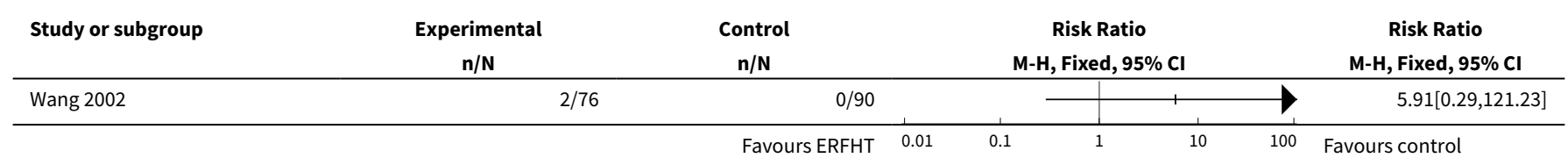

Comparison 13. Laser therapy versus medical treatment

\begin{tabular}{lllll}
\hline Outcome or subgroup title & No. of studies & $\begin{array}{l}\text { No. of partici- } \\
\text { pants }\end{array}$ & Statistical method & Effect size \\
\hline 1 Prostatitis symptoms & 1 & & Risk Ratio (M-H, Fixed, 95\% Cl) & Totals not selected \\
\hline 2 Adverse events & 1 & 112 & Odds Ratio (M-H, Fixed, 95\% Cl) & $0.0[0.0,0.0]$ \\
\hline
\end{tabular}


Analysis 13.1. Comparison 13 Laser therapy versus medical treatment, Outcome 1 Prostatitis symptoms.

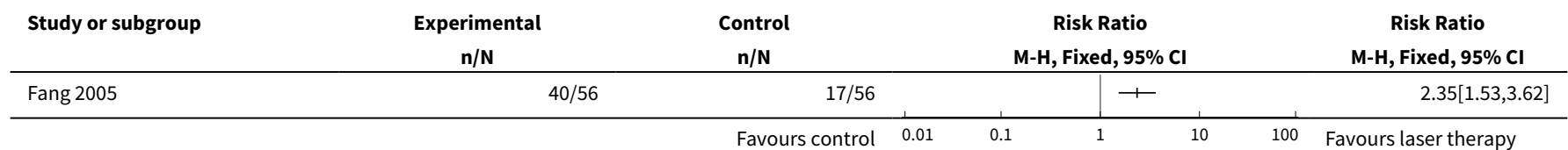

Analysis 13.2. Comparison 13 Laser therapy versus medical treatment, Outcome 2 Adverse events.

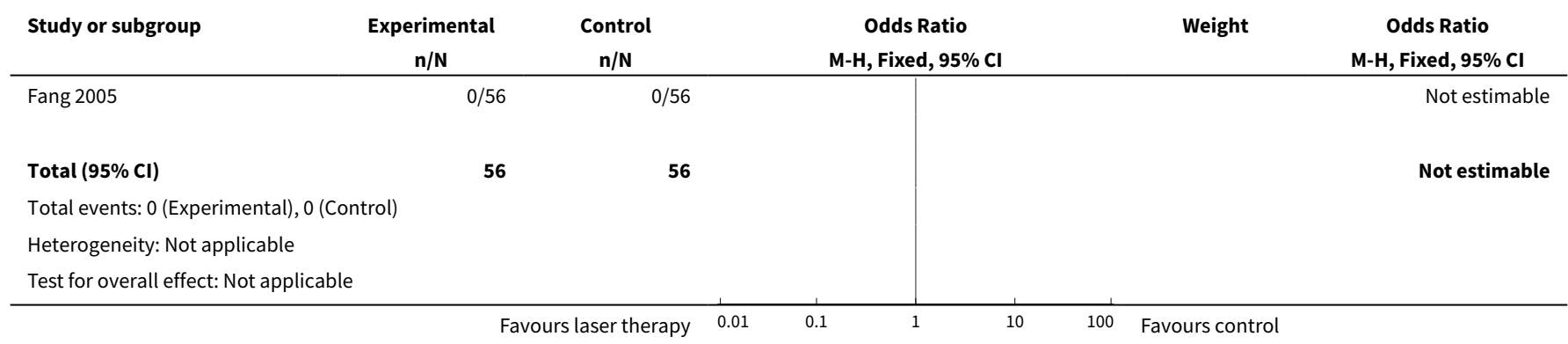

\section{Comparison 14. Tibial nerve stimulation versus no intervention}

\begin{tabular}{lllll}
\hline Outcome or subgroup title & No. of studies & $\begin{array}{l}\text { No. of partici- } \\
\text { pants }\end{array}$ & Statistical method & Effect size \\
\hline $\begin{array}{l}1 \text { Prostatitis symptoms (NIH-CPSI } \\
\text { total) }\end{array}$ & 1 & 89 & $\begin{array}{l}\text { Mean Difference (IV, Fixed, } \\
95 \% \mathrm{CI})\end{array}$ & $-11.2[-12.92,-9.48]$ \\
\hline $\begin{array}{l}\text { P Prostatitis symptoms: pain sub- } \\
\text { score }\end{array}$ & 1 & 89 & $\begin{array}{l}\text { Mean Difference (IV, Fixed, } \\
95 \% \mathrm{CI})\end{array}$ & $-5.70[-6.90,-4.50]$ \\
\hline $\begin{array}{l}\text { 3 Prostatitis symptoms: micturition } \\
\text { subscore }\end{array}$ & 1 & 89 & $\begin{array}{l}\text { Mean Difference (IV, Fixed, } \\
95 \% \mathrm{CI})\end{array}$ & $-3.20[-3.77,-2.63]$ \\
\hline $\begin{array}{l}\text { 4 Prostatitis symptoms: quality of } \\
\text { life subscore }\end{array}$ & 1 & 89 & $\begin{array}{l}\text { Mean Difference (IV, Fixed, } \\
95 \% \mathrm{CI})\end{array}$ & $-4.6[-5.27,-3.93]$ \\
\hline
\end{tabular}

\section{Analysis 14.1. Comparison 14 Tibial nerve stimulation versus no} intervention, Outcome 1 Prostatitis symptoms (NIH-CPSI total).

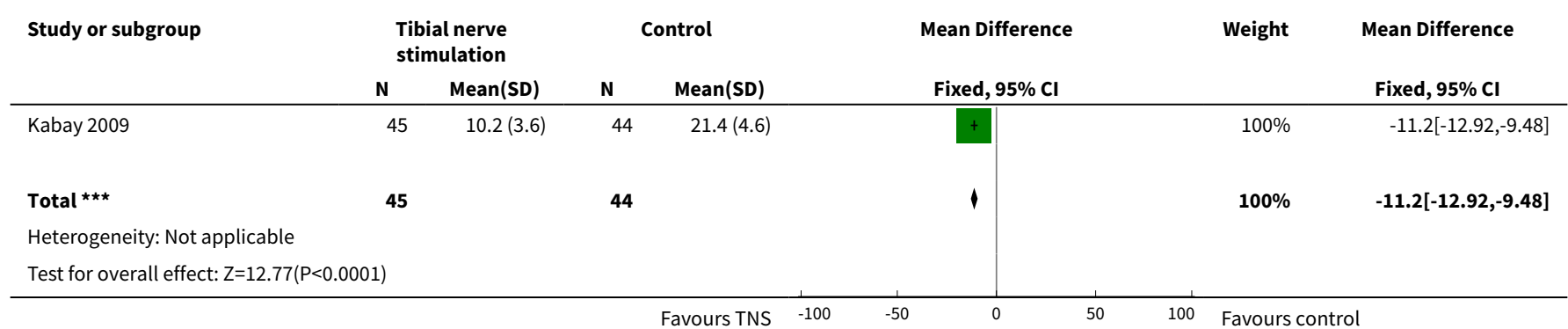




\section{Analysis 14.2. Comparison 14 Tibial nerve stimulation versus no} intervention, Outcome 2 Prostatitis symptoms: pain subscore.

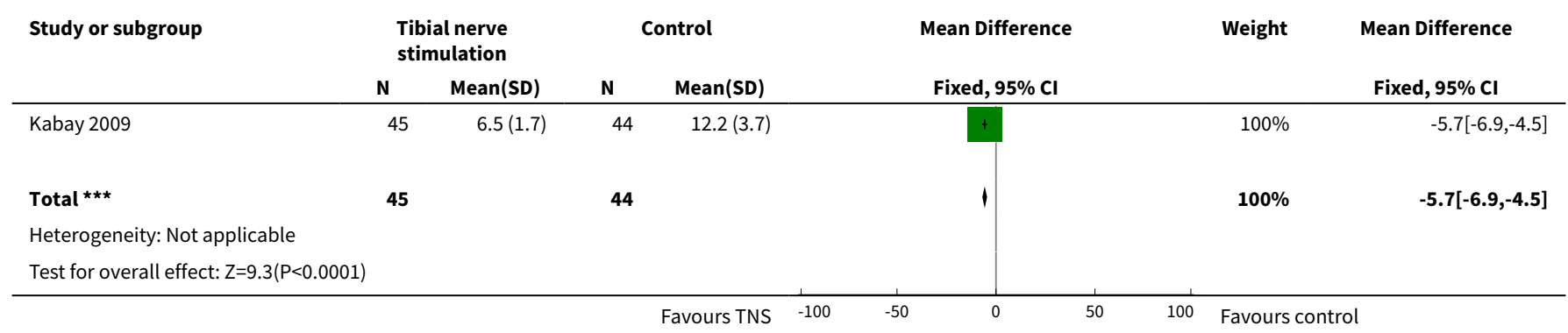

Analysis 14.3. Comparison 14 Tibial nerve stimulation versus no intervention, Outcome 3 Prostatitis symptoms: micturition subscore.

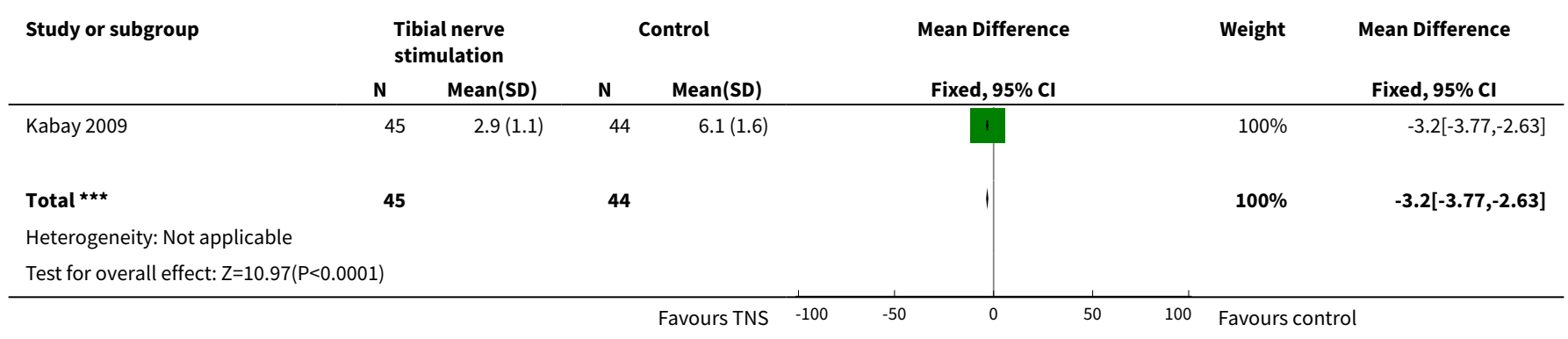

Analysis 14.4. Comparison 14 Tibial nerve stimulation versus no intervention, Outcome 4 Prostatitis symptoms: quality of life subscore.

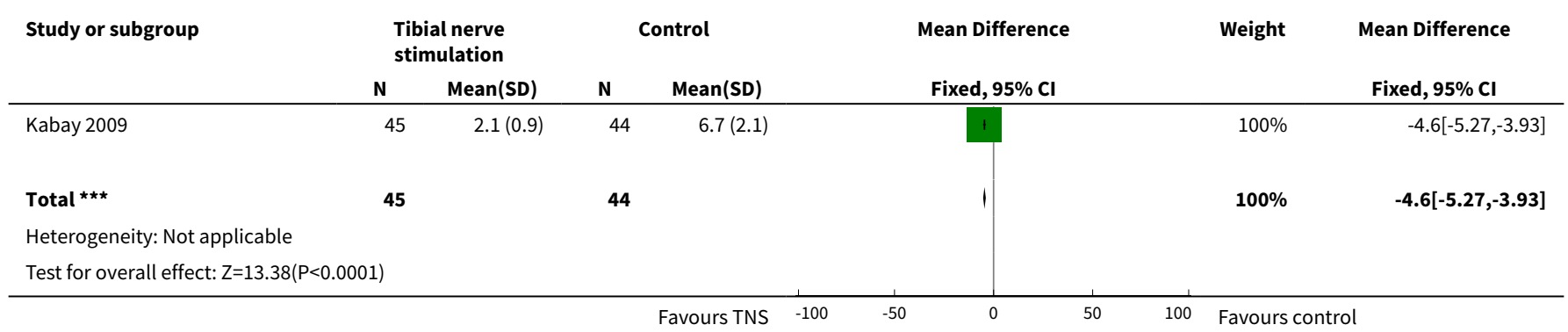

Comparison 15. Myofascial therapy versus control intervention

\begin{tabular}{lllll}
\hline Outcome or subgroup title & No. of studies & $\begin{array}{l}\text { No. of partici- } \\
\text { pants }\end{array}$ & Statistical method & Effect size \\
\hline $\begin{array}{l}1 \text { Prostatitis symptoms (NIH- } \\
\text { CPSI total) }\end{array}$ & 1 & 21 & $\begin{array}{l}\text { Mean Difference (IV, Fixed, 95\% } \\
\text { Cl) }\end{array}$ & $1.0[-6.45,8.45]$ \\
\hline
\end{tabular}




\begin{tabular}{|c|c|c|c|c|}
\hline Outcome or subgroup title & No. of studies & $\begin{array}{l}\text { No. of partici- } \\
\text { pants }\end{array}$ & Statistical method & Effect size \\
\hline $\begin{array}{l}2 \text { Prostatitis symptoms: pain } \\
\text { subscore }\end{array}$ & 1 & 21 & $\begin{array}{l}\text { Mean Difference (IV, Fixed, 95\% } \\
\mathrm{CI})\end{array}$ & $-0.20[-5.04,4.64]$ \\
\hline $\begin{array}{l}3 \text { Prostatitis symptoms: micturi- } \\
\text { tion subscore }\end{array}$ & 1 & 21 & $\begin{array}{l}\text { Mean Difference (IV, Fixed, 95\% } \\
\mathrm{CI})\end{array}$ & $1.1[-1.12,3.32]$ \\
\hline $\begin{array}{l}4 \text { Prostatitis symptoms: quality } \\
\text { of life subscore }\end{array}$ & 1 & 21 & $\begin{array}{l}\text { Mean Difference (IV, Fixed, 95\% } \\
\mathrm{CI})\end{array}$ & $0.10[-2.90,3.10]$ \\
\hline 5 Sexual dysfunction & 1 & 21 & $\begin{array}{l}\text { Mean Difference (IV, Fixed, 95\% } \\
\mathrm{CI})\end{array}$ & $-2.20[-9.24,4.84]$ \\
\hline 6 Quality of life - physical & 1 & & $\begin{array}{l}\text { Mean Difference (IV, Fixed, 95\% } \\
\mathrm{CI})\end{array}$ & Totals not selected \\
\hline 7 Quality of life - mental & 1 & 19 & $\begin{array}{l}\text { Mean Difference (IV, Fixed, 95\% } \\
\mathrm{CI} \text { ) }\end{array}$ & $0.80[-9.25,10.85]$ \\
\hline
\end{tabular}

Analysis 15.1. Comparison 15 Myofascial therapy versus control intervention, Outcome 1 Prostatitis symptoms (NIH-CPSI total).

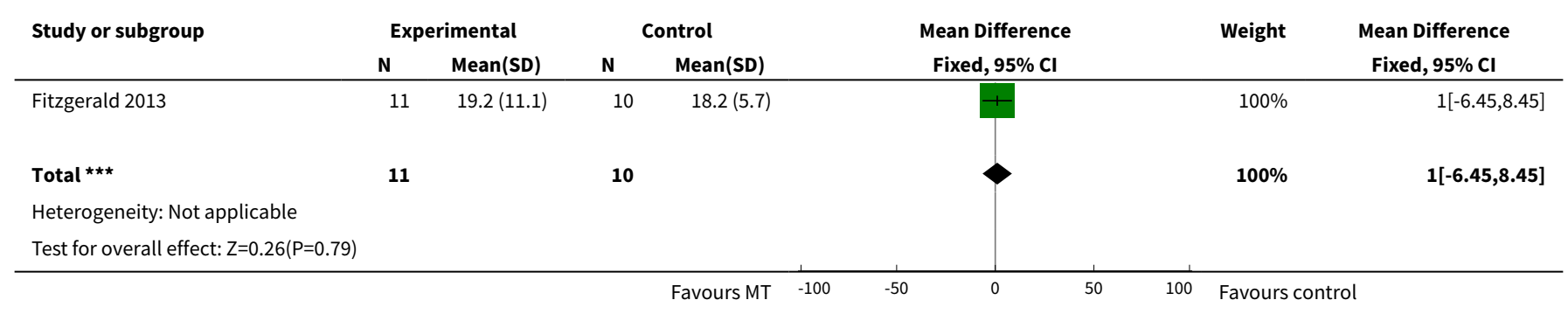

\section{Analysis 15.2. Comparison 15 Myofascial therapy versus control} intervention, Outcome 2 Prostatitis symptoms: pain subscore.

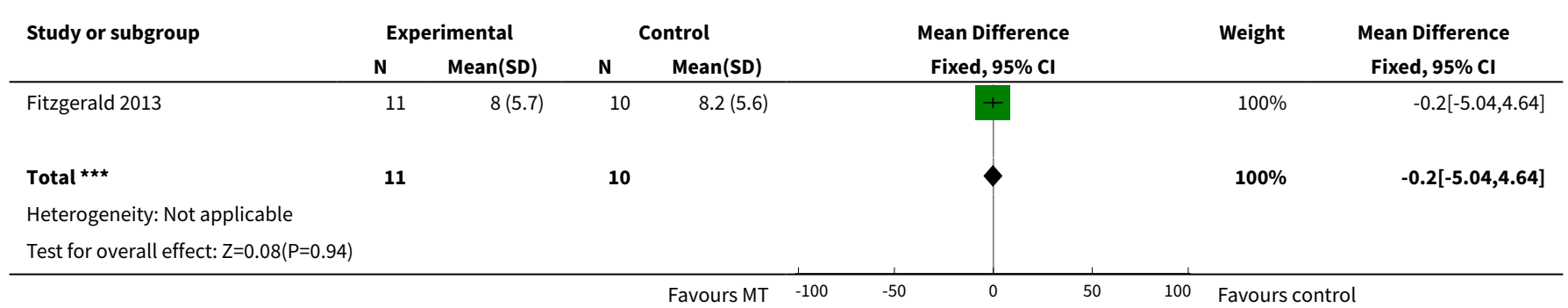


Analysis 15.3. Comparison 15 Myofascial therapy versus control intervention, Outcome 3 Prostatitis symptoms: micturition subscore.

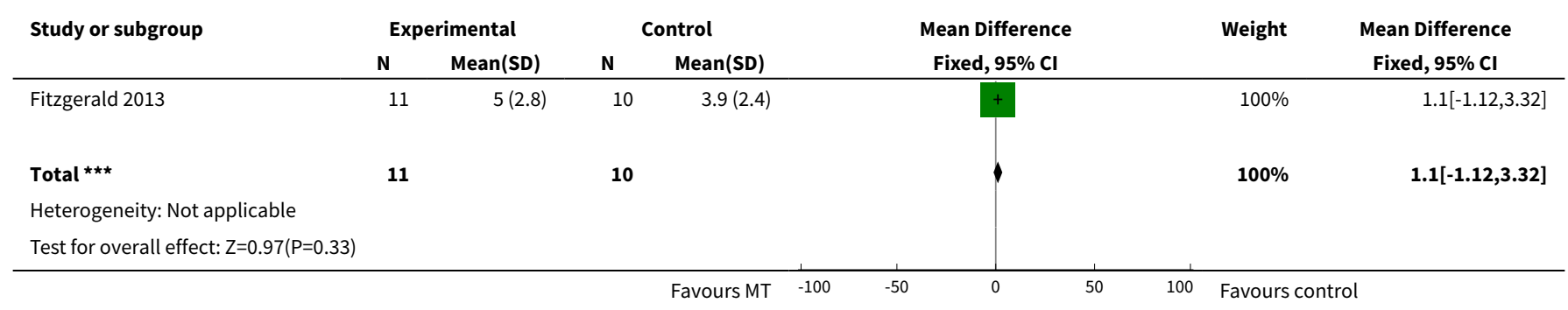

Analysis 15.4. Comparison 15 Myofascial therapy versus control intervention, Outcome 4 Prostatitis symptoms: quality of life subscore.

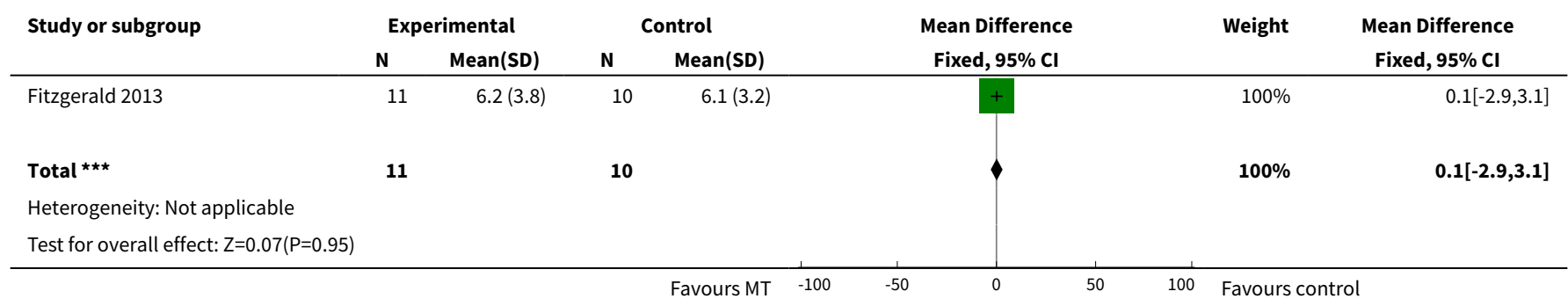

Analysis 15.5. Comparison 15 Myofascial therapy versus control intervention, Outcome 5 Sexual dysfunction.

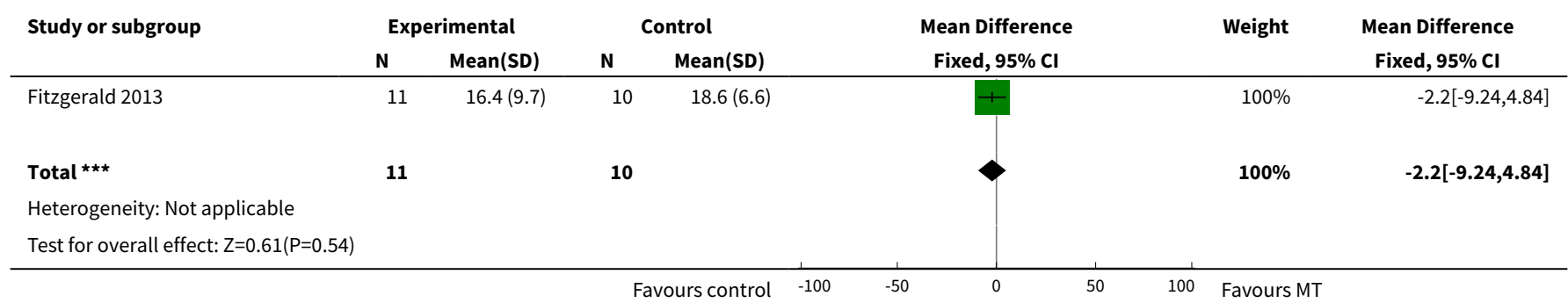

Analysis 15.6. Comparison 15 Myofascial therapy versus control intervention, Outcome 6 Quality of life - physical.

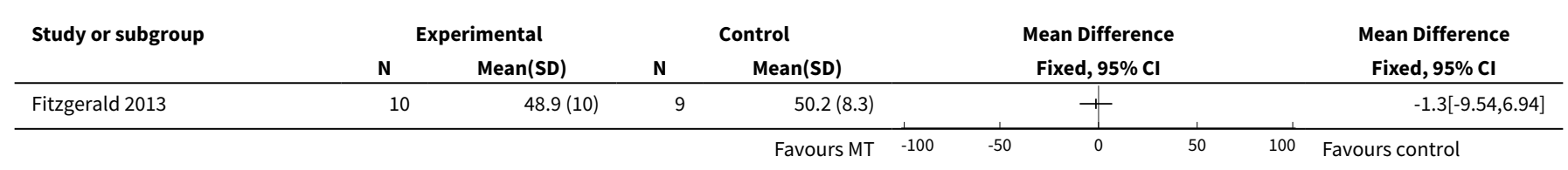


Analysis 15.7. Comparison 15 Myofascial therapy versus control intervention, Outcome 7 Quality of life - mental.

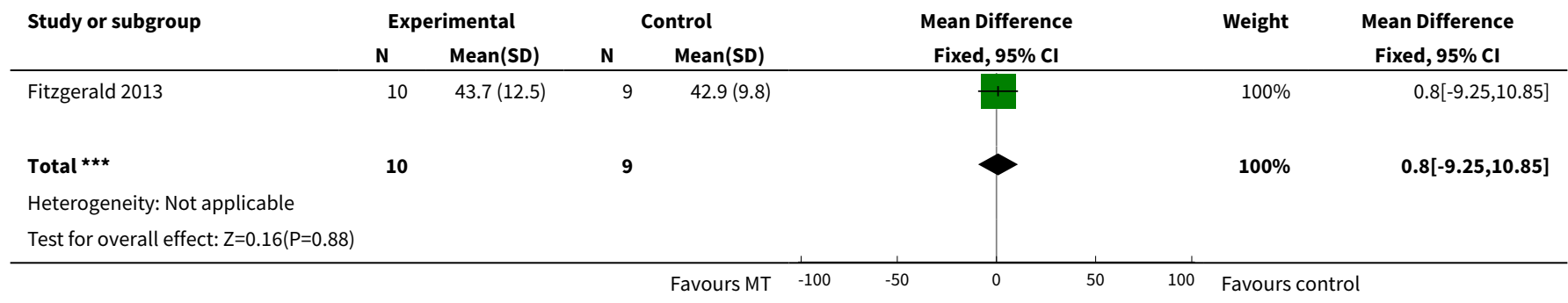

Comparison 16. Osteopathy versus sham procedure

\begin{tabular}{lllll}
\hline Outcome or subgroup title & No. of studies & $\begin{array}{l}\text { No. of partici- } \\
\text { pants }\end{array}$ & Statistical method & Effect size \\
\hline $\begin{array}{l}1 \text { Prostatitis symptoms (NIH-CPSI } \\
\text { total) }\end{array}$ & 1 & $\begin{array}{l}\text { Mean Difference (IV, Fixed, 95\% } \\
\text { Cl) }\end{array}$ & Totals not selected \\
\hline $\begin{array}{l}2 \text { Prostatitis symptoms: quality of } \\
\text { life subscore }\end{array}$ & 1 & $\begin{array}{l}\text { Mean Difference (IV, Fixed, 95\% } \\
\text { Cl) }\end{array}$ & Totals not selected \\
\hline $\begin{array}{l}\text { U Urinary symptoms } \\
\text { n }\end{array}$ & 1 & $\begin{array}{l}\text { Mean Difference (IV, Fixed, 95\% } \\
\text { Cl) }\end{array}$ & Totals not selected \\
\hline
\end{tabular}

Analysis 16.1. Comparison 16 Osteopathy versus sham procedure, Outcome 1 Prostatitis symptoms (NIH-CPSI total).

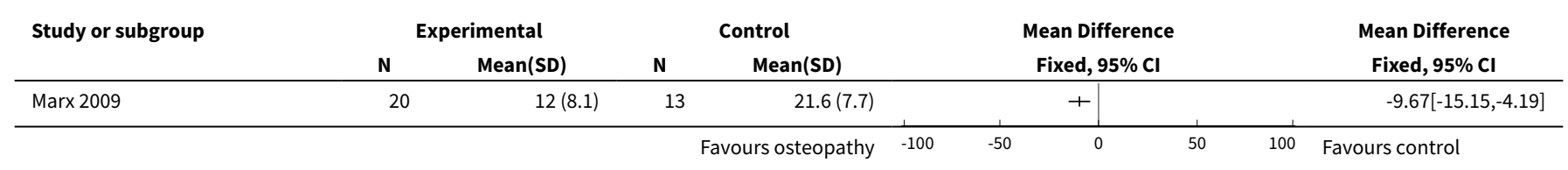

Analysis 16.2. Comparison 16 Osteopathy versus sham procedure, Outcome 2 Prostatitis symptoms: quality of life subscore.

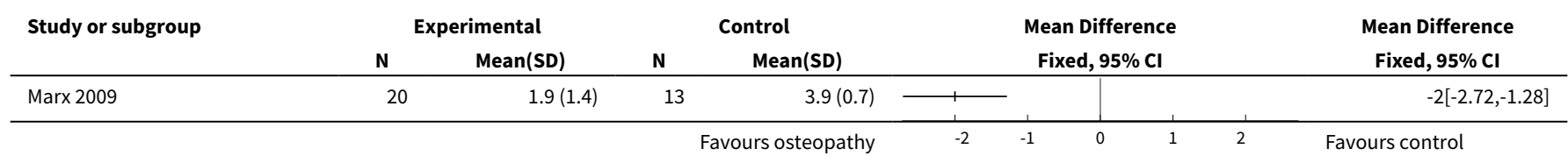


Analysis 16.3. Comparison 16 Osteopathy versus sham procedure, Outcome 3 Urinary symptoms.

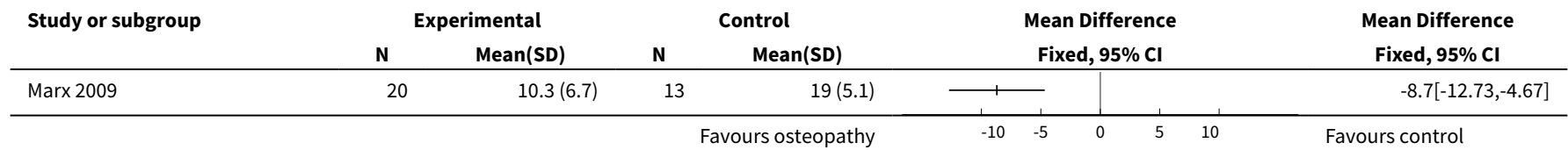

Comparison 17. Sono-electromagnetic therapy versus placebo

\begin{tabular}{lllll}
\hline Outcome or subgroup title & No. of studies & $\begin{array}{l}\text { No. of partici- } \\
\text { pants }\end{array}$ & Statistical method & Effect size \\
\hline $\begin{array}{l}1 \text { Prostatitis symptoms (NIH-CPSI to- } \\
\text { tal) }\end{array}$ & 1 & 60 & $\begin{array}{l}\text { Mean Difference (IV, Fixed, } \\
95 \% \text { CI) }\end{array}$ & -2.80 [-6.75, 1.15] \\
\hline 2 Prostatitis symptoms & 1 & 60 & $\begin{array}{l}\text { Risk Ratio (M-H, Fixed, 95\% } \\
\text { CI) }\end{array}$ & 1.4 [0.91, 2.15] \\
\hline $\begin{array}{l}\text { 3 Prostatitis symptoms: pain sub- } \\
\text { score }\end{array}$ & 1 & 60 & $\begin{array}{l}\text { Mean Difference (IV, Fixed, } \\
95 \% \text { Cl) }\end{array}$ & $-1.30[-3.44,0.84]$ \\
\hline $\begin{array}{l}\text { 4 Prostatitis symptoms: micturition } \\
\text { subscore }\end{array}$ & 1 & 60 & $\begin{array}{l}\text { Mean Difference (IV, Fixed, } \\
95 \% \text { Cl) }\end{array}$ & 0.0 [-1.26, 1.26] \\
\hline $\begin{array}{l}\text { 5 Prostatitis symptoms: quality of life } \\
\text { subscore }\end{array}$ & 1 & 60 & $\begin{array}{l}\text { Mean Difference (IV, Fixed, } \\
95 \% \text { Cl) }\end{array}$ & -1.40 [-2.76, -0.04] \\
\hline
\end{tabular}

Analysis 17.1. Comparison 17 Sono-electromagnetic therapy versus placebo, Outcome 1 Prostatitis symptoms (NIH-CPSI total).

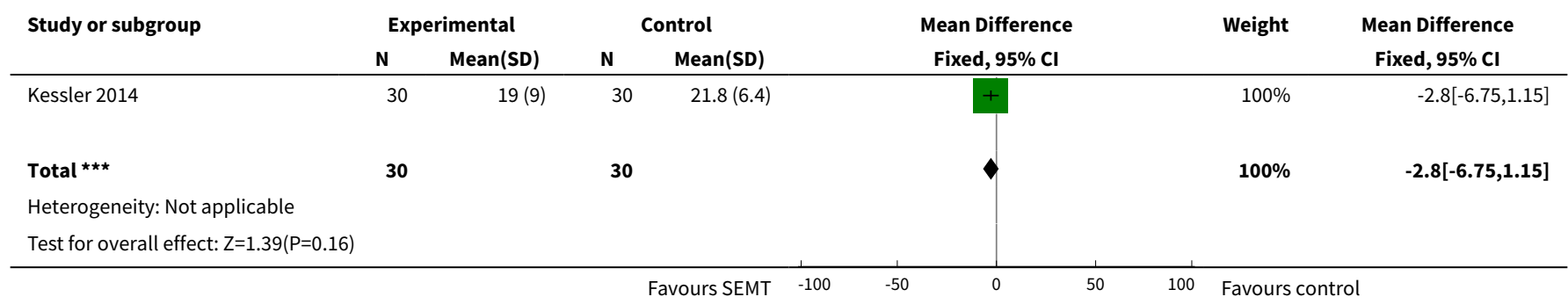

\section{Analysis 17.2. Comparison 17 Sono-electromagnetic therapy versus placebo, Outcome 2 Prostatitis symptoms.}

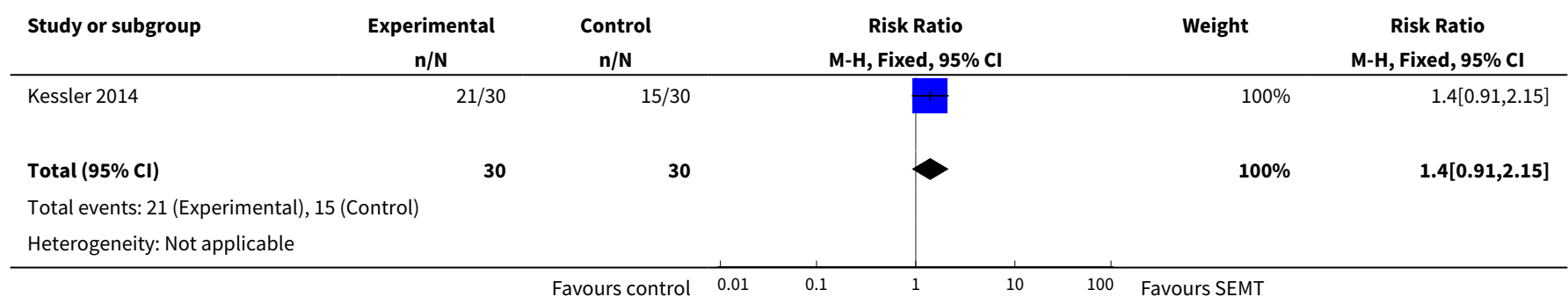




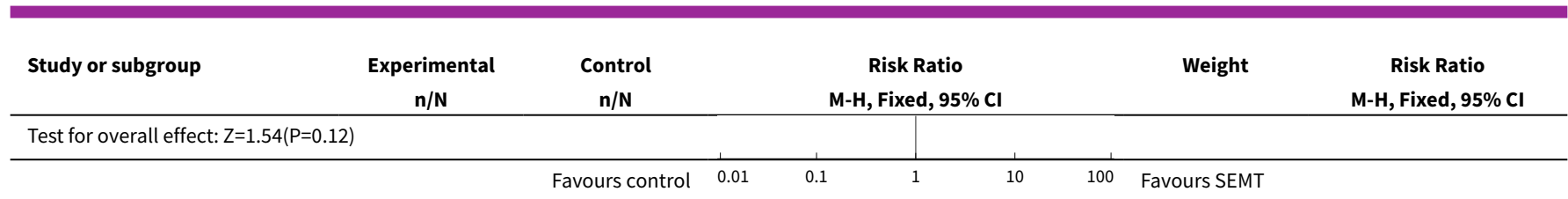

Analysis 17.3. Comparison 17 Sono-electromagnetic therapy versus placebo, Outcome 3 Prostatitis symptoms: pain subscore.

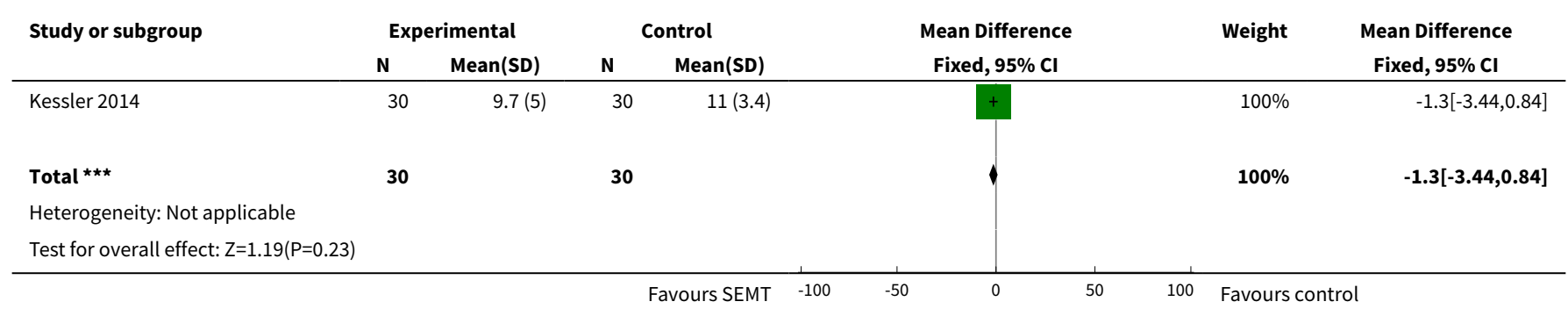

Analysis 17.4. Comparison 17 Sono-electromagnetic therapy versus placebo, Outcome 4 Prostatitis symptoms: micturition subscore.

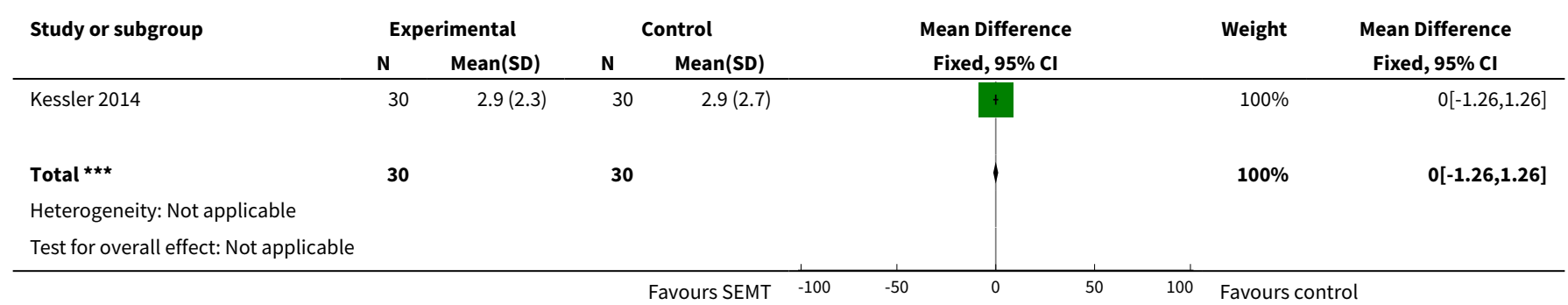

Analysis 17.5. Comparison 17 Sono-electromagnetic therapy versus placebo, Outcome 5 Prostatitis symptoms: quality of life subscore.

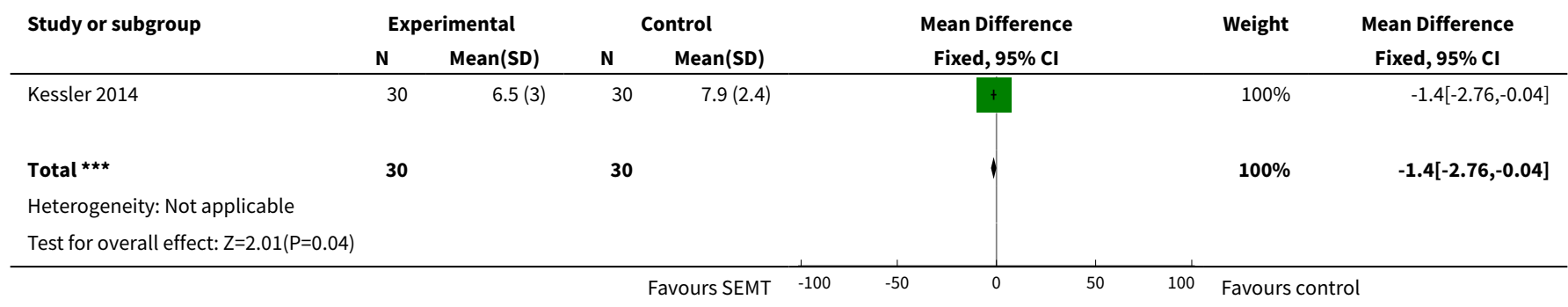


Comparison 18. Transelectrical nerve stimulation (TENS) versus control

\begin{tabular}{|c|c|c|c|c|}
\hline Outcome or subgroup title & No. of studies & $\begin{array}{l}\text { No. of partici- } \\
\text { pants }\end{array}$ & Statistical method & Effect size \\
\hline $\begin{array}{l}1 \text { Prostatitis symptoms: pain } \\
\text { subscore }\end{array}$ & 2 & & $\begin{array}{l}\text { Mean Difference (IV, Fixed, 95\% } \\
\mathrm{CI} \text { ) }\end{array}$ & Subtotals only \\
\hline $\begin{array}{l}\text { 1.1 Compared to sham proce- } \\
\text { dure }\end{array}$ & 1 & 40 & $\begin{array}{l}\text { Mean Difference (IV, Fixed, 95\% } \\
\mathrm{CI} \text { ) }\end{array}$ & $\begin{array}{l}-15.25[-17.71 \\
-12.79]\end{array}$ \\
\hline 1.2 Compared to no intervention & 1 & 16 & $\begin{array}{l}\text { Mean Difference (IV, Fixed, 95\% } \\
\text { CI) }\end{array}$ & $-6.88[-8.13,-5.63]$ \\
\hline
\end{tabular}

Analysis 18.1. Comparison 18 Transelectrical nerve stimulation (TENS) versus control, Outcome 1 Prostatitis symptoms: pain subscore.

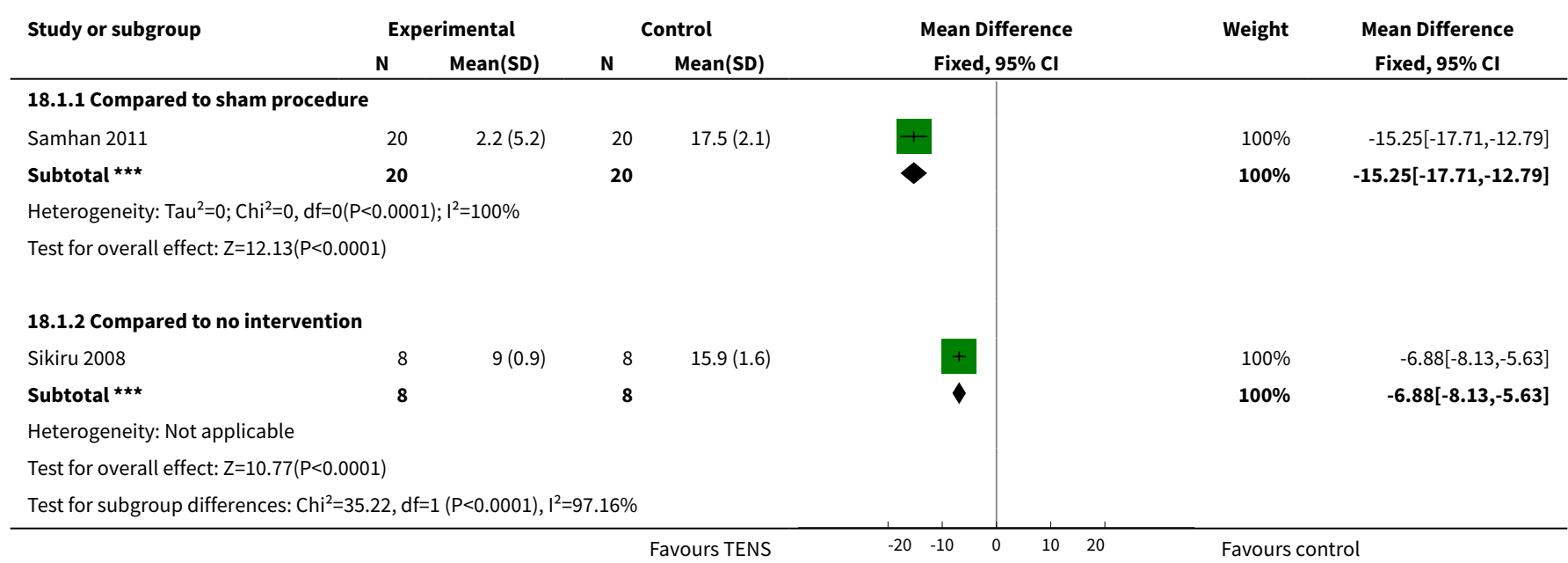

\section{Comparison 19. Transurethral microwave thermotherapy}

\begin{tabular}{|c|c|c|c|c|}
\hline Outcome or subgroup title & No. of studies & $\begin{array}{l}\text { No. of partici- } \\
\text { pants }\end{array}$ & Statistical method & Effect size \\
\hline $1.170^{\circ} \mathrm{C}$ vs $55^{\circ} \mathrm{C}$ & 1 & 39 & Mean Difference (IV, Fixed, 95\% CI) & $-1.10[-6.50,4.30]$ \\
\hline 1.2 Compared to control & 1 & 20 & Mean Difference (IV, Fixed, 95\% CI) & $0.0[0.0,0.0]$ \\
\hline $\begin{array}{l}2 \text { Prostatitis symptoms: pain } \\
\text { subscore }\end{array}$ & 1 & & Mean Difference (IV, Fixed, 95\% CI) & Totals not selected \\
\hline $\begin{array}{l}3 \text { Prostatitis symptoms: mic- } \\
\text { turition subscore }\end{array}$ & 1 & & Mean Difference (IV, Fixed, 95\% CI) & Totals not selected \\
\hline
\end{tabular}




\begin{tabular}{lllll}
\hline Outcome or subgroup title & No. of studies & $\begin{array}{l}\text { No. of partici- } \\
\text { pants }\end{array}$ & Statistical method & Effect size \\
\hline $\begin{array}{l}\text { 4 Prostatitis symptoms: quali- } \\
\text { ty of life subscore }\end{array}$ & 1 & & Mean Difference (IV, Fixed, 95\% Cl) & Totals not selected \\
\hline 5 Urinary symptoms & 2 & Mean Difference (IV, Fixed, 95\% Cl) & Subtotals only \\
\hline $5.170{ }^{\circ} \mathrm{C}$ vs $55{ }^{\circ} \mathrm{C}$ & 1 & Mean Difference (IV, Fixed, 95\% Cl) & $-2.10[-6.34,2.14]$ \\
\hline 5.2 Compared to control & 1 & 20 & Mean Difference (IV, Fixed, 95\% Cl) & $0.0[0.0,0.0]$ \\
\hline
\end{tabular}

Analysis 19.1. Comparison 19 Transurethral microwave thermotherapy, Outcome 1 Prostatitis symptoms (NIH-CPSI total).

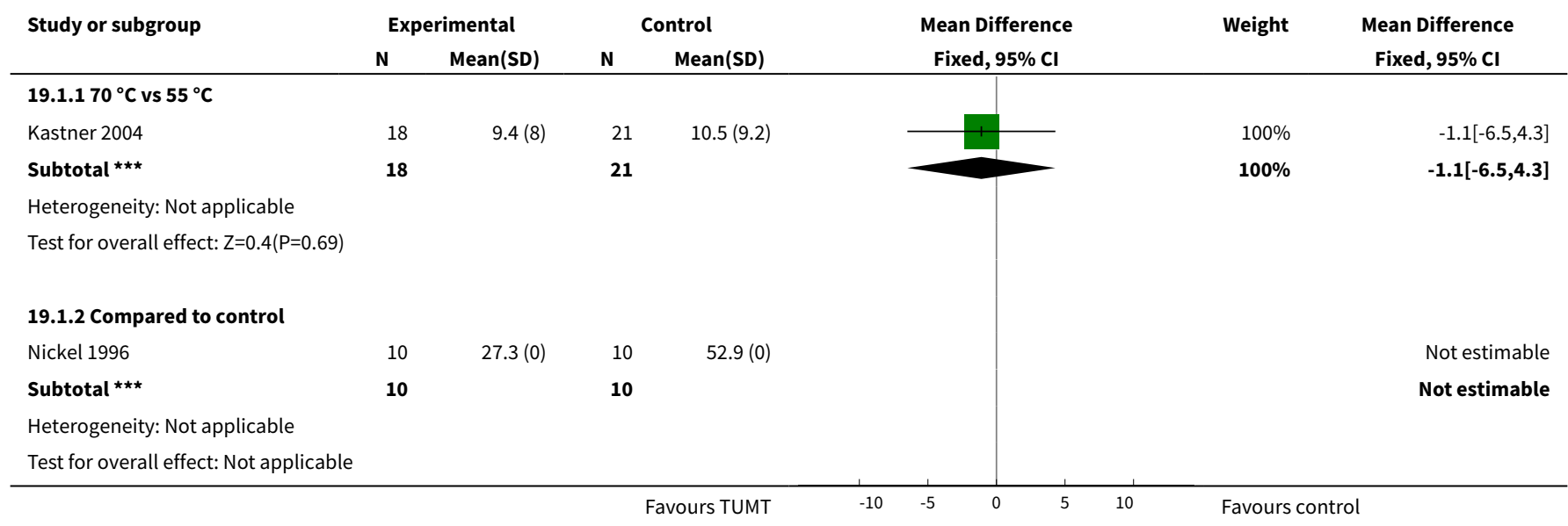

Analysis 19.2. Comparison 19 Transurethral microwave thermotherapy, Outcome 2 Prostatitis symptoms: pain subscore.

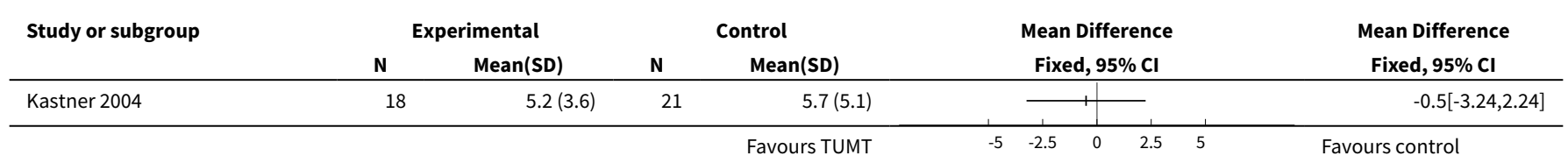

Analysis 19.3. Comparison 19 Transurethral microwave thermotherapy, Outcome 3 Prostatitis symptoms: micturition subscore.

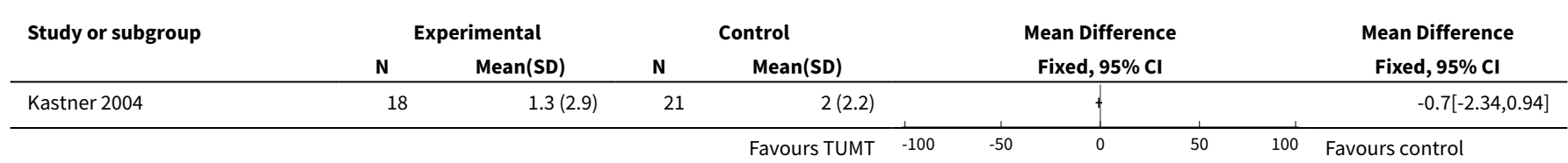


Analysis 19.4. Comparison 19 Transurethral microwave thermotherapy, Outcome 4 Prostatitis symptoms: quality of life subscore.

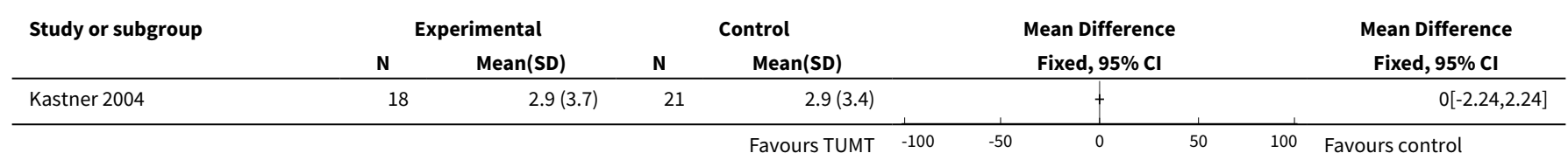

Analysis 19.5. Comparison 19 Transurethral microwave thermotherapy, Outcome 5 Urinary symptoms.

\begin{tabular}{|c|c|c|c|c|c|c|c|c|}
\hline \multirow[t]{2}{*}{ Study or subgroup } & \multicolumn{2}{|c|}{ Experimental } & \multicolumn{2}{|c|}{ Control } & & \multirow{2}{*}{$\begin{array}{c}\text { Mean Difference } \\
\text { Fixed, } 95 \% \mathrm{Cl}\end{array}$} & \multirow[t]{2}{*}{ Weight } & \multirow{2}{*}{$\begin{array}{c}\text { Mean Difference } \\
\text { Fixed, } 95 \% \mathrm{Cl}\end{array}$} \\
\hline & $\mathbf{N}$ & Mean(SD) & $\mathbf{N}$ & Mean(SD) & & & & \\
\hline \multicolumn{9}{|l|}{ 19.5.1 $70^{\circ} \mathrm{C}$ vs $55^{\circ} \mathrm{C}$} \\
\hline Kastner 2004 & 18 & $4.5(6.1)$ & 21 & $6.6(7.4)$ & & + & $100 \%$ & $-2.1[-6.34,2.14]$ \\
\hline Subtotal $* \star \star$ & 18 & & 21 & & & & $100 \%$ & $-2.1[-6.34,2.14]$ \\
\hline \multicolumn{9}{|c|}{ Heterogeneity: Not applicable } \\
\hline \multicolumn{9}{|c|}{ Test for overall effect: $Z=0.97(P=0.33)$} \\
\hline \multicolumn{9}{|c|}{ 19.5.2 Compared to control } \\
\hline Nickel 1996 & 10 & $12.8(0)$ & 10 & $21.9(0)$ & & & & Not estimable \\
\hline Subtotal $\star \star \star ~$ & 10 & & 10 & & & & & Not estimable \\
\hline \multicolumn{9}{|c|}{ Heterogeneity: Not applicable } \\
\hline \multicolumn{9}{|c|}{ Test for overall effect: Not applicable } \\
\hline & & & & ours TUMT & -100 & -50 & $100 \quad$ Favour & \\
\hline
\end{tabular}

Comparison 20. Transurethral needle ablation (TUNA) versus sham procedure

\begin{tabular}{lllll}
\hline Outcome or subgroup title & No. of studies & $\begin{array}{l}\text { No. of partici- } \\
\text { pants }\end{array}$ & Statistical method & Effect size \\
\hline $\begin{array}{l}\text { 1 Prostatitis symptoms (NIH- } \\
\text { CPSI total) }\end{array}$ & 1 & 33 & Mean Difference (IV, Fixed, 95\% Cl) & $2.30[-8.02,12.62]$ \\
\hline 2 Urinary symptoms & 1 & 33 & Mean Difference (IV, Fixed, 95\% Cl) & $0.40[-5.09,5.89]$ \\
\hline
\end{tabular}

Analysis 20.1. Comparison 20 Transurethral needle ablation (TUNA) versus sham procedure, Outcome 1 Prostatitis symptoms (NIH-CPSI total).

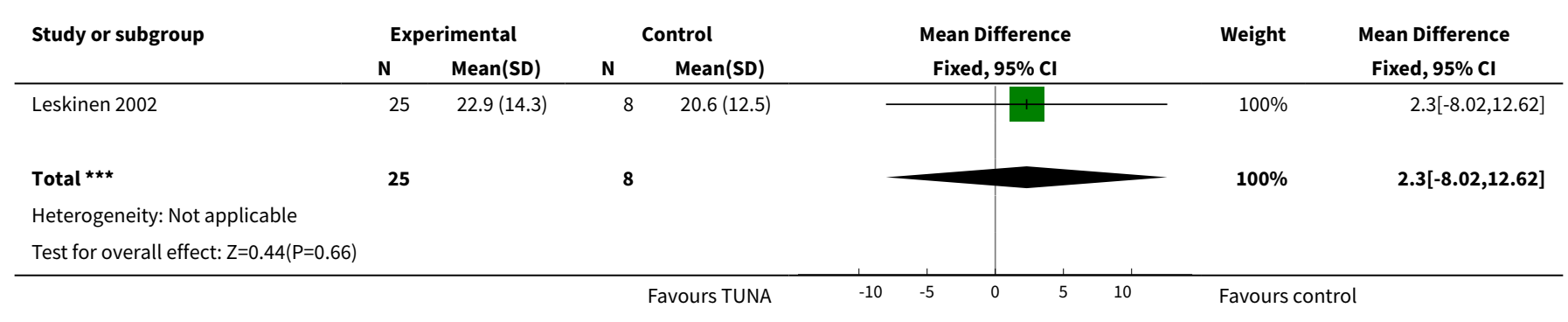


Analysis 20.2. Comparison 20 Transurethral needle ablation (TUNA) versus sham procedure, Outcome 2 Urinary symptoms.

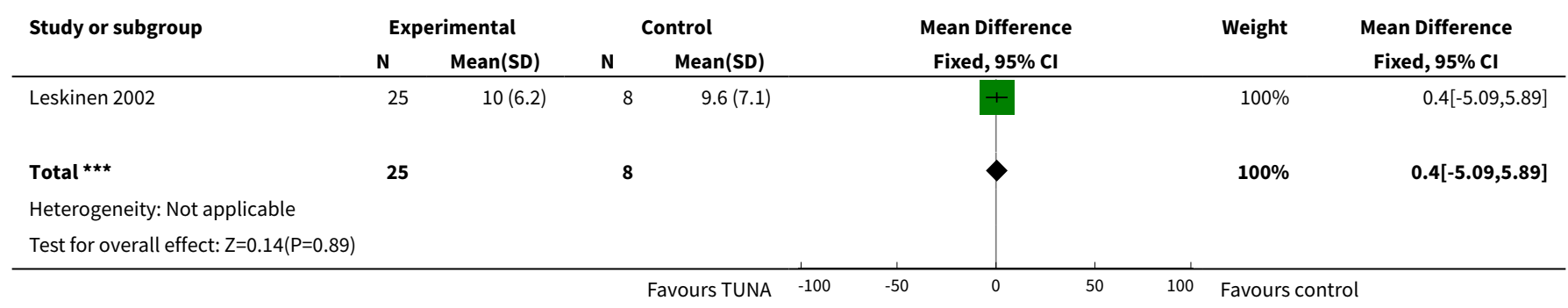

\section{Comparison 21. Ultrasound (non-intrusive)}

\begin{tabular}{|c|c|c|c|c|}
\hline Outcome or subgroup title & No. of studies & $\begin{array}{l}\text { No. of partici- } \\
\text { pants }\end{array}$ & Statistical method & Effect size \\
\hline 1 Prostatitis symptoms (NIH-CPSI total) & 1 & & $\begin{array}{l}\text { Mean Difference (IV, Fixed, } \\
95 \% \mathrm{Cl})\end{array}$ & Subtotals only \\
\hline 1.1 Ultrasound vs medical therapy & 1 & 70 & $\begin{array}{l}\text { Mean Difference (IV, Fixed, } \\
95 \% \mathrm{Cl})\end{array}$ & $1.09[0.16,2.02]$ \\
\hline $\begin{array}{l}1.2 \text { Ultrasound in combination with } \\
\text { medical therapy vs medical therapy } \\
\text { alone }\end{array}$ & 1 & 70 & $\begin{array}{l}\text { Mean Difference (IV, Fixed, } \\
95 \% \mathrm{CI})\end{array}$ & $\begin{array}{l}-6.67[-7.62 \\
-5.72]\end{array}$ \\
\hline 2 Prostatitis symptoms: pain subscore & 1 & & $\begin{array}{l}\text { Mean Difference (IV, Fixed, } \\
95 \% \mathrm{Cl} \text { ) }\end{array}$ & $\begin{array}{l}\text { Totals not select- } \\
\text { ed }\end{array}$ \\
\hline 2.1 Ultrasound vs medical therapy & 1 & & $\begin{array}{l}\text { Mean Difference (IV, Fixed, } \\
95 \% \mathrm{Cl})\end{array}$ & $0.0[0.0,0.0]$ \\
\hline $\begin{array}{l}2.2 \text { Ultrasound in combination with } \\
\text { medical therapy vs medical therapy } \\
\text { alone }\end{array}$ & 1 & & $\begin{array}{l}\text { Mean Difference (IV, Fixed, } \\
95 \% \mathrm{Cl})\end{array}$ & $0.0[0.0,0.0]$ \\
\hline $\begin{array}{l}3 \text { Prostatitis symptoms: micturition sub- } \\
\text { score }\end{array}$ & 1 & & $\begin{array}{l}\text { Mean Difference (IV, Fixed, } \\
95 \% \mathrm{CI})\end{array}$ & $\begin{array}{l}\text { Totals not select- } \\
\text { ed }\end{array}$ \\
\hline 3.1 Ultrasound vs medical therapy & 1 & & $\begin{array}{l}\text { Mean Difference (IV, Fixed, } \\
95 \% \mathrm{CI})\end{array}$ & $0.0[0.0,0.0]$ \\
\hline $\begin{array}{l}3.2 \text { Ultrasound in combination with } \\
\text { medical therapy vs medical therapy } \\
\text { alone }\end{array}$ & 1 & & $\begin{array}{l}\text { Mean Difference (IV, Fixed, } \\
95 \% \mathrm{Cl} \text { ) }\end{array}$ & $0.0[0.0,0.0]$ \\
\hline $\begin{array}{l}4 \text { Prostatitis symptoms: quality of life } \\
\text { subscore }\end{array}$ & 1 & & $\begin{array}{l}\text { Mean Difference (IV, Fixed, } \\
95 \% \mathrm{Cl})\end{array}$ & $\begin{array}{l}\text { Totals not select- } \\
\text { ed }\end{array}$ \\
\hline 4.1 Ultrasound vs medical therapy & 1 & & $\begin{array}{l}\text { Mean Difference (IV, Fixed, } \\
95 \% \mathrm{Cl} \text { ) }\end{array}$ & $0.0[0.0,0.0]$ \\
\hline
\end{tabular}




\begin{tabular}{|c|c|c|c|c|}
\hline Outcome or subgroup title & No. of studies & $\begin{array}{l}\text { No. of partici- } \\
\text { pants }\end{array}$ & Statistical method & Effect size \\
\hline $\begin{array}{l}4.2 \text { Ultrasound in combination with } \\
\text { medical therapy vs medical therapy } \\
\text { alone }\end{array}$ & 1 & & $\begin{array}{l}\text { Mean Difference (IV, Fixed, } \\
95 \% \mathrm{CI})\end{array}$ & $0.0[0.0,0.0]$ \\
\hline 5 Prostatitis symptoms & 1 & & $\begin{array}{l}\text { Risk Ratio (M-H, Fixed, 95\% } \\
\mathrm{Cl} \text { ) }\end{array}$ & $\begin{array}{l}\text { Totals not select- } \\
\text { ed }\end{array}$ \\
\hline 5.1 Ultrasound vs medical therapy & 1 & & $\begin{array}{l}\text { Risk Ratio (M-H, Fixed, 95\% } \\
\mathrm{Cl})\end{array}$ & $0.0[0.0,0.0]$ \\
\hline $\begin{array}{l}5.2 \text { Ultrasound in combination with } \\
\text { medical therapy vs medical therapy } \\
\text { alone }\end{array}$ & 1 & & $\begin{array}{l}\text { Risk Ratio (M-H, Fixed, 95\% } \\
\mathrm{Cl})\end{array}$ & $0.0[0.0,0.0]$ \\
\hline
\end{tabular}

Analysis 21.1. Comparison 21 Ultrasound (non-intrusive), Outcome 1 Prostatitis symptoms (NIH-CPSI total).

\begin{tabular}{|c|c|c|c|c|c|c|c|}
\hline \multirow[t]{2}{*}{ Study or subgroup } & \multicolumn{2}{|c|}{ Experimental } & \multicolumn{2}{|c|}{ Control } & \multirow{2}{*}{$\begin{array}{l}\text { Mean Difference } \\
\text { Fixed, } 95 \% \mathrm{Cl}\end{array}$} & \multirow[t]{2}{*}{ Weight } & \multirow{2}{*}{$\begin{array}{l}\text { Mean Difference } \\
\text { Fixed, } 95 \% \mathrm{Cl}\end{array}$} \\
\hline & $\mathbf{N}$ & Mean(SD) & $\mathbf{N}$ & Mean(SD) & & & \\
\hline \multicolumn{8}{|c|}{ 21.1.1 Ultrasound vs medical therapy } \\
\hline Kaikai 2014 & 35 & $19.7(2.1)$ & 35 & $18.6(1.9)$ & & $100 \%$ & $1.09[0.16,2.02]$ \\
\hline 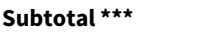 & 35 & & 35 & & & $100 \%$ & $1.09[0.16,2.02]$ \\
\hline \multicolumn{8}{|c|}{ Heterogeneity: Not applicable } \\
\hline \multicolumn{8}{|c|}{ Test for overall effect: $Z=2.31(P=0.02)$} \\
\hline \multicolumn{8}{|c|}{$\begin{array}{l}\text { 21.1.2 Ultrasound in combination with medical therapy vs medical therapy } \\
\text { alone }\end{array}$} \\
\hline Kaikai 2014 & 35 & $11.9(2.2)$ & 35 & $18.6(1.9)$ & & $100 \%$ & $-6.67[-7.62,-5.72]$ \\
\hline Subtotal $\star \star \star$ & 35 & & 35 & & & $100 \%$ & $-6.67[-7.62,-5.72]$ \\
\hline \multicolumn{8}{|c|}{ Heterogeneity: Not applicable } \\
\hline \multicolumn{8}{|c|}{ Test for overall effect: $Z=13.74(P<0.0001)$} \\
\hline
\end{tabular}

Analysis 21.2. Comparison 21 Ultrasound (non-intrusive), Outcome 2 Prostatitis symptoms: pain subscore.

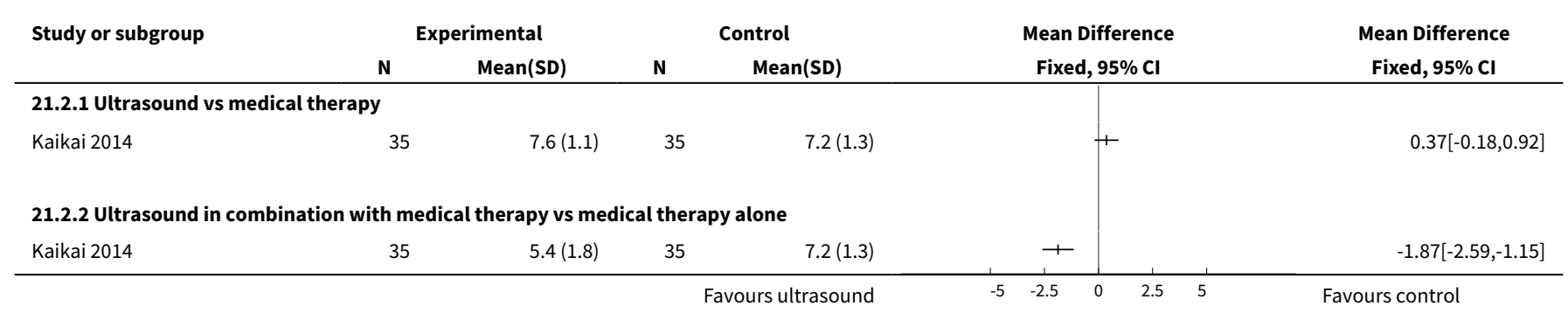


Analysis 21.3. Comparison 21 Ultrasound (non-intrusive), Outcome 3 Prostatitis symptoms: micturition subscore.

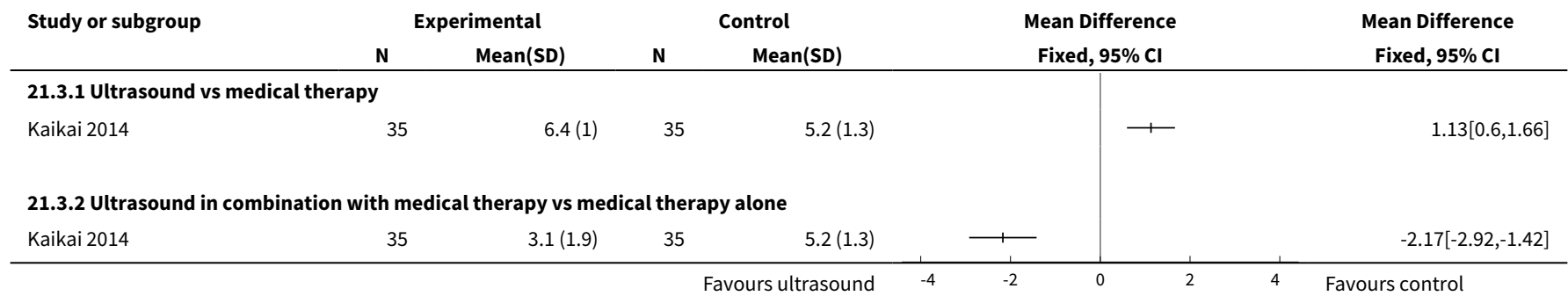

Analysis 21.4. Comparison 21 Ultrasound (non-intrusive), Outcome 4 Prostatitis symptoms: quality of life subscore.

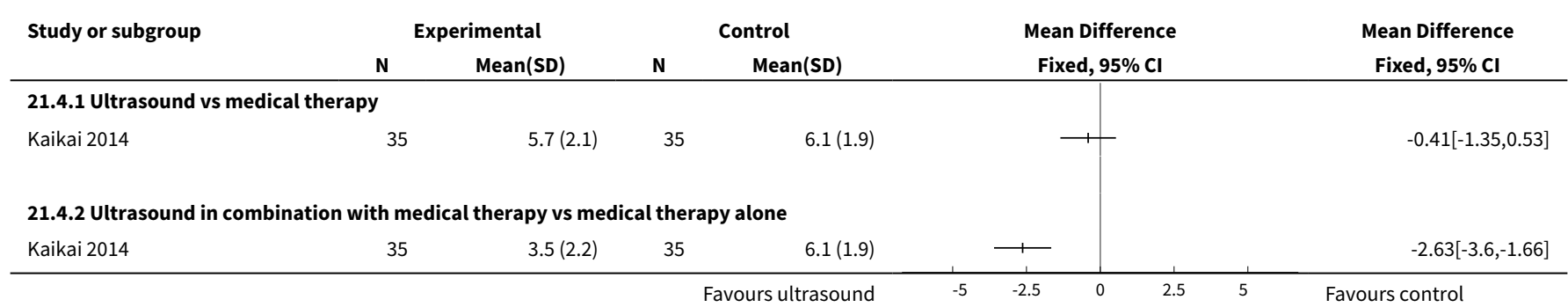

Analysis 21.5. Comparison 21 Ultrasound (non-intrusive), Outcome 5 Prostatitis symptoms.

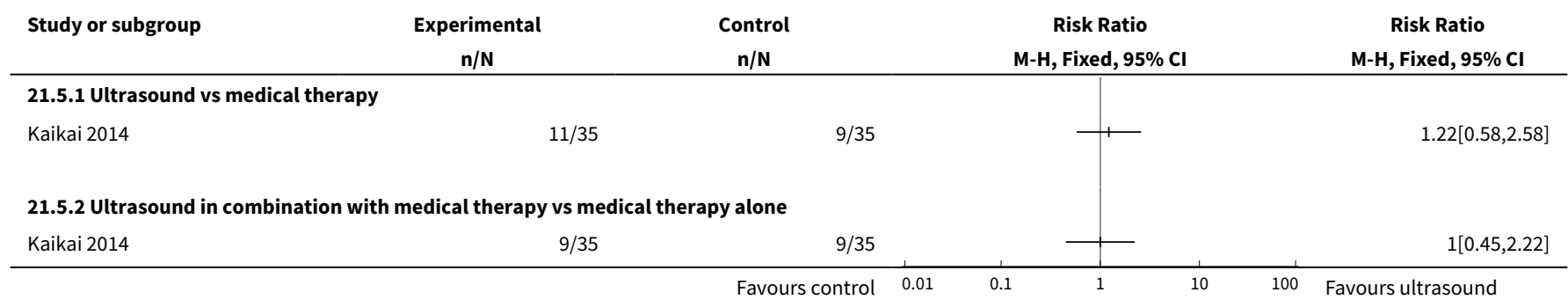

\section{Comparison 22. Acupuncture treatments versus medical treatment - sensitivity analysis}

\begin{tabular}{lllll}
\hline Outcome or subgroup title & No. of studies & $\begin{array}{l}\text { No. of partici- } \\
\text { pants }\end{array}$ & Statistical method & Effect size \\
\hline $\begin{array}{l}\text { 1 Prostatitis symptoms } \\
\text { (NIH-CPSI total) }\end{array}$ & 2 & 78 & $\begin{array}{l}\text { Mean Difference (IV, Random, 95\% } \\
\text { CI) }\end{array}$ & $-6.05[-7.87,-4.24]$ \\
\hline $\begin{array}{llll}1.1 \text { Acupuncture } \\
\text { I.2 Electroacupuncture }\end{array}$ & 1 & 54 & $\begin{array}{l}\text { Mean Difference (IV, Random, 95\% } \\
\text { CI) }\end{array}$ & $-6.11[-8.75,-3.47]$ \\
\hline
\end{tabular}


Analysis 22.1. Comparison 22 Acupuncture treatments versus medical treatment - sensitivity analysis, Outcome 1 Prostatitis symptoms (NIH-CPSI total).

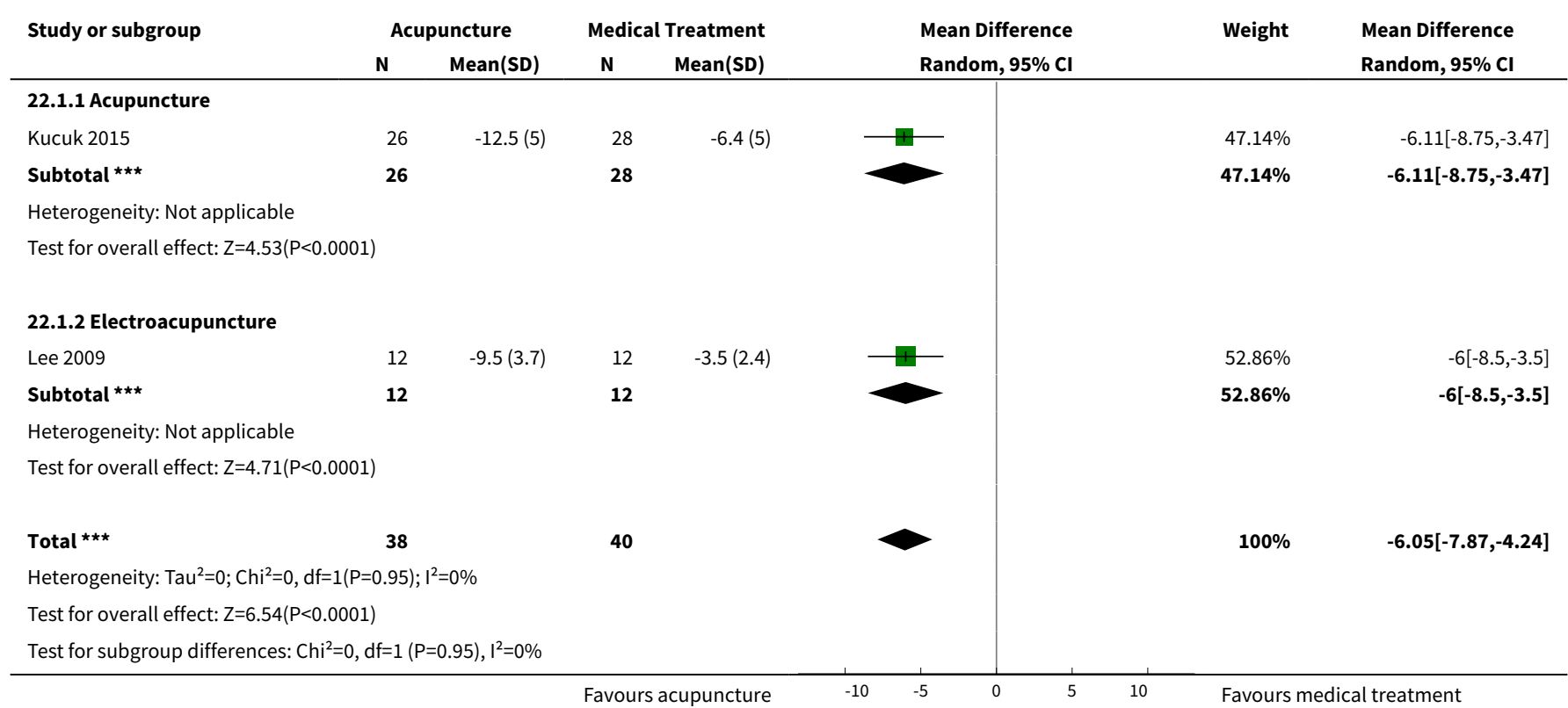

\section{ADDITIONAL TABLES}

Table 1. Description of interventions

\begin{tabular}{|c|c|c|c|c|c|c|}
\hline Study ID & $\begin{array}{l}\text { Intervention(s) (route, fre- } \\
\text { quency, total dose/day) }\end{array}$ & $\begin{array}{l}\text { Interven- } \\
\text { tion(s) appro- } \\
\text { priate as ap- } \\
\text { plied in a clin- } \\
\text { ical practice } \\
\text { setting (de- } \\
\text { scription) }\end{array}$ & $\begin{array}{l}\text { Interven- } \\
\text { tion(s) du- } \\
\text { ration }\end{array}$ & $\begin{array}{l}\text { Comparator(s) } \\
\text { (route, frequen- } \\
\text { cy, total dose/ } \\
\text { day) }\end{array}$ & $\begin{array}{l}\text { Compara- } \\
\text { tor(s) ap- } \\
\text { propriate as } \\
\text { applied in a } \\
\text { clinical prac- } \\
\text { tice setting } \\
\text { (description) }\end{array}$ & $\begin{array}{l}\text { Compara- } \\
\text { tor(s) du- } \\
\text { ration }\end{array}$ \\
\hline $\begin{array}{l}\text { Neimark } \\
2016\end{array}$ & $\begin{array}{l}\text { Breathing exercises using 'Kar- } \\
\text { bonik' apparatus (hypercap- } \\
\text { nic hypoxia) 10-20 min daily + } \\
\text { medical therapy (see compari- } \\
\text { son). }\end{array}$ & $\begin{array}{l}\text { No information } \\
\text { regarding dose- } \\
\text { scaling or con- } \\
\text { traindications. }\end{array}$ & 10 days. & $\begin{array}{l}\text { Levofloxacin } 500 \\
\text { mg/day, tam- } \\
\text { sulosin } 0.4 \mathrm{mg} / \\
\text { day, Samprost } \\
\text { daily supposi- } \\
\text { tory, Serenoa } \\
\text { repens fructu- } \\
\text { um extract } 1 \text { cap- } \\
\text { sule/day for } 10 \\
\text { days, nimesulide } \\
1-2 \text { tablets/day } \\
\text { for } 5-7 \text { days. }\end{array}$ & $\begin{array}{l}\text { No informa- } \\
\text { tion regarding } \\
\text { dose-scaling } \\
\text { or contraindi- } \\
\text { cations. }\end{array}$ & 10 days. \\
\hline $\begin{array}{l}\text { Pajovic } \\
2016\end{array}$ & $\begin{array}{l}\text { ESWT + medical therapy: } \\
\text { each session had 12-min du- } \\
\text { ration and } 3000 \text { impulses with } \\
\text { total energy flow } 0.25 \mathrm{~mJ} / \\
\mathrm{mm}^{2} 3 \mathrm{~Hz} \text {, weekly in supine } \\
\text { position without anaesthesia. }\end{array}$ & $\begin{array}{l}\text { No information } \\
\text { regarding dose- } \\
\text { scaling or con- } \\
\text { traindications. }\end{array}$ & 12 weeks. & $\begin{array}{l}\text { Medical therapy } \\
\text { with doxazosin } 4 \\
\mathrm{mg} \text { daily, ibupro- } \\
\text { fen } 400 \mathrm{mg} \text { daily } \\
\text { and tiocolchico- } \\
\text { side } 12 \mathrm{mg} \text { daily. }\end{array}$ & $\begin{array}{l}\text { Ranitidine } \\
\text { was allowed } \\
\text { for gastric } \\
\text { complaints. } \\
\text { No informa- } \\
\text { tion regarding }\end{array}$ & 12 weeks. \\
\hline
\end{tabular}

Non-pharmacological interventions for treating chronic prostatitis/chronic pelvic pain syndrome (Review) 
Table 1. Description of interventions (Continued)

dose-scaling or contraindications.

\begin{tabular}{ll}
\hline Kucuk & Acupuncture group: \\
2015 & UB28 bladder meridian \\
& GB41 gallbladder meridian \\
LIV3 liver 3 meridian \\
LI4 large intestine 4 meridian \\
SP6 spleen 6 meridian \\
SP8 spleen 8 meridian \\
Stimulation using dispos- \\
able acupuncture needles \\
(Hua Long, 25 40 mm Sterile \\
Acupuncture Needles, China) \\
and electrical pulse generator \\
(Agistim Duo, 44 mA rms max/ \\
99 Hz max, France).
\end{tabular}

\section{Sahin 2015 Acupuncture, using 2 dispos-} able stainless steel needles $0.3 \mathrm{~mm}$ diameter, $60 \mathrm{~mm}$ length, Suzhou, Jiangsu, China) that were inserted to a depth of maximum $2.5-3 \mathrm{~cm}$ in 7 acupoints bilaterally: BL33 Zhongliao

BL34 Xialiao

BL54 Zhibian

CV1 Huiyin

CV4 Guanyuan

SP6 Sanyinjiao

SP9 Yinlingquan. Duration: 20 min (with rotation).

$\begin{array}{lll}\begin{array}{l}\text { No information } \\ \text { regarding dose- }\end{array} & 7 \text { weeks. } & \begin{array}{l}\text { Medical thera- } \\ \text { py levofloxacin } \\ \text { scaling. }\end{array} \\ \begin{array}{l}500 \mathrm{mg} \text { daily and } \\ \text { ibuprofen } 200\end{array} \\ \text { "Localized } & \mathrm{mg} .\end{array}$

tions concern-

ing the acu-

points, bleed-

ing diathe-

sis and use of

anticoagula-

tion" were con-

traindications.
Orally admin- 7 weeks. istrated twice daily for 7 weeks.

No information regarding dose-scaling or contraindications.

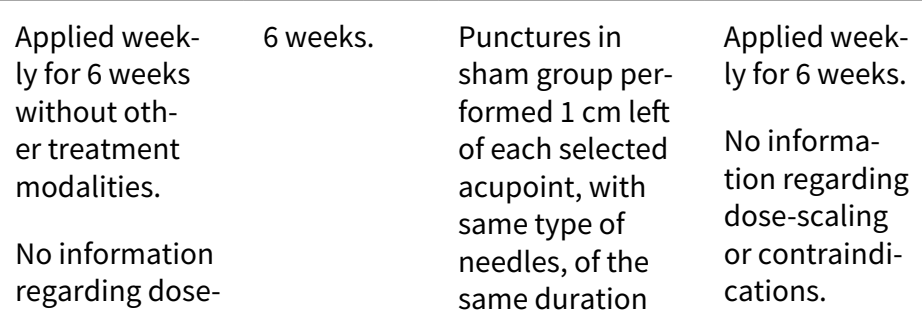
scaling or contraindications. and frequency. cations.
6 weeks. (n)

\begin{tabular}{|c|c|c|c|c|c|c|}
\hline Zhao 2015 & $\begin{array}{l}\text { Medical therapy (4 weeks of } \\
\text { ciprofloxacin } 500 \text { mg bid, } 3 \\
\text { months of ibuprofen } 400 \mathrm{mg} / \\
\text { day, } 3 \text { months of tamsulosin } \\
0.4 \mathrm{mg} / \text { day) and scheduled } \\
\text { for surgery during the same } \\
\text { period in each of the sites by } \\
\text { study clinicians; given written } \\
\text { instructions about postopera- } \\
\text { tive wound care. }\end{array}$ & $\begin{array}{l}\text { No information } \\
\text { regarding dose- } \\
\text { scaling or con- } \\
\text { traindications. }\end{array}$ & 3 months. & $\begin{array}{l}\text { Same medica- } \\
\text { tions ( } 4 \text { weeks } \\
\text { of ciprofloxacin } \\
500 \mathrm{mg} \text { bid, } \\
3 \text { months of } \\
\text { ibuprofen } 400 \\
\mathrm{mg} / \mathrm{day}, 3 \\
\text { months of tam- } \\
\text { sulosin } 0.4 \mathrm{mg} / \\
\text { day) and remain } \\
\text { uncircumcised } \\
\text { until end of } 3- \\
\text { month study par- } \\
\text { ticipation, when } \\
\text { they were sched- } \\
\text { uled to undergo } \\
\text { circumcision. }\end{array}$ & $\begin{array}{l}\text { No informa- } \\
\text { tion regarding } \\
\text { dose-scaling } \\
\text { or contraindi- } \\
\text { cations. }\end{array}$ & 3 months. \\
\hline Gallo 2014 & $\begin{array}{l}\text { Individually discussed risk fac- } \\
\text { tors detected at history by } \\
\text { the completed questionnaire, } \\
\text { informed that such risk fac- } \\
\text { tors were potential causes of } \\
\text { their disease symptoms and }\end{array}$ & $\begin{array}{l}\text { No information } \\
\text { regarding dose- } \\
\text { scaling or con- } \\
\text { traindications. }\end{array}$ & 3 months. & $\begin{array}{l}\text { Followed same } \\
\text { diet, sexual be- } \\
\text { haviours and } \\
\text { lifestyle as those } \\
\text { of the previous } \\
\text { months. }\end{array}$ & $\begin{array}{l}\text { No informa- } \\
\text { tion regarding } \\
\text { dose-scaling } \\
\text { or contraindi- } \\
\text { cations. }\end{array}$ & 3 months. \\
\hline
\end{tabular}


Table 1. Description of interventions (Continued)

it was strongly recommended to avoid them, distributed a vademecum with 13 rules relating to diet, sexual habits and lifestyle.

\begin{tabular}{|c|c|c|c|c|c|c|}
\hline $\begin{array}{l}\text { Kessler } \\
2014\end{array}$ & $\begin{array}{l}\text { Sono-electromagnetic ther- } \\
\text { apy at home using Sonodyn } \\
\text { Device: intensity } 100 \mathrm{~mW} / \mathrm{cm}^{2} \\
\text { with ultrasonic power of } 12 \\
\mathrm{~mW} \text { and frequency } 1.9 \mathrm{MHz} \text {, } \\
\text { electric field force } 0.3 \mathrm{~V} / \mathrm{m} \text { and } \\
\text { magnetic field force } 0.4 \mathrm{~A} / \mathrm{m} \text {; } \\
\text { for } 10 \text { min, twice daily. }\end{array}$ & $\begin{array}{l}\text { Participants } \\
\text { could not see } \\
\text { settings or per- } \\
\text { ceive the de- } \\
\text { vice. No in- } \\
\text { formation re- } \\
\text { garding dose- } \\
\text { scaling or con- } \\
\text { traindications. }\end{array}$ & 12 weeks. & $\begin{array}{l}\text { Similar placebo } \\
\text { device. Partic- } \\
\text { ipants applied } \\
\text { same procedure, } \\
\text { but device was } \\
\text { not active during } \\
\text { session. }\end{array}$ & $\begin{array}{l}\text { Participants } \\
\text { could not see } \\
\text { settings or } \\
\text { perceive de- } \\
\text { vice. No in- } \\
\text { formation re- } \\
\text { garding dose- } \\
\text { scaling or con- } \\
\text { traindications. }\end{array}$ & 12 weeks. \\
\hline $\begin{array}{l}\text { Kaikai } \\
2014\end{array}$ & $\begin{array}{l}\text { Non-intrusive ultrasound. Out- } \\
\text { put frequency } 1.79 \mathrm{MHz} \text {; out- } \\
\text { put power: } 3.15 \mathrm{~W} / \mathrm{cm}^{2} \text {. Du- } \\
\text { ration: } 20 \mathrm{~min} \text {, administered } \\
\text { every } 3 \text { days (total of } 7 \text { times) } \\
\text { + integrated Chinese-Western } \\
\text { medications. }\end{array}$ & $\begin{array}{l}\text { No information } \\
\text { regarding dose- } \\
\text { scaling or con- } \\
\text { traindications. }\end{array}$ & - & $\begin{array}{l}\text { Integrated Chi- } \\
\text { nese-Western } \\
\text { medications } \\
\text { alone; Qian- } \\
\text { LieShuTong Cap- } \\
\text { sule, orally } 3 \\
\text { times daily, } 3 \\
\text { capsules each } \\
\text { time, tamsulosin } \\
\text { delayed-release } \\
\text { capsule, } 0.2 \text { mg, } \\
\text { orally } 4 \text { times } \\
\text { daily. }\end{array}$ & $\begin{array}{l}\text { No informa- } \\
\text { tion regarding } \\
\text { dose-scaling } \\
\text { or contraindi- } \\
\text { cations. }\end{array}$ & 1 month. \\
\hline $\begin{array}{l}\text { Vahdat- } \\
\text { pour } 2013\end{array}$ & $\begin{array}{l}\text { ESWT (DUOLITH SD1, Storz } \\
\text { Medical, Tägerwilen, Switzer- } \\
\text { land) once weekly for } 4 \text { weeks. } \\
\text { Each time } 3000 \text { impulses, } \\
\text { with } 0.25 \mathrm{~mJ} / \mathrm{mm}^{2} \text { and } 3 \mathrm{~Hz}\end{array}$ & $\begin{array}{l}\text { No information } \\
\text { regarding dose- } \\
\text { scaling or con- } \\
\text { traindications. }\end{array}$ & 4 weeks. & $\begin{array}{l}\text { In sham group, } \\
\text { same proto- } \\
\text { col applied but } \\
\text { with probe being } \\
\text { turned off. }\end{array}$ & $\begin{array}{l}\text { No informa- } \\
\text { tion regarding } \\
\text { dose-scaling } \\
\text { or contraindi- } \\
\text { cations. }\end{array}$ & 4 weeks. \\
\hline
\end{tabular}
of frequency were delivered, although $0.5 \mathrm{~mJ} / \mathrm{mm}^{2}$ was added in each week $(0.3 \mathrm{~mJ} /$ $\mathrm{mm}^{2}$ in week $2,0.35 \mathrm{~mJ} / \mathrm{mm}^{2}$ in week 3 and $0.4 \mathrm{~mJ} / \mathrm{mm}^{2}$ in week 4). After each 500 pulses, probe position was corrected, using transperineal ultrasound.

\begin{tabular}{|c|c|c|c|c|c|}
\hline $\begin{array}{l}\text { Fitzgerald } \\
2013\end{array}$ & $\begin{array}{l}10 \times 1 \text {-hour sessions of my- } \\
\text { ofascial physical therapy in- } \\
\text { volved connective tissue ma- } \\
\text { nipulation of the abdominal } \\
\text { wall, back, buttocks and thighs } \\
\text { with connective tissue abnor- } \\
\text { malities in prone and supine } \\
\text { position; double voiding and } \\
\text { squatting (as home exercises); } \\
\text { transrectal manipulation. }\end{array}$ & $\begin{array}{l}\text { No information } \\
\text { regarding dose- } \\
\text { scaling. } \\
\text { "The presence } \\
\text { of painful scars } \\
\text { on lower ab- } \\
\text { dominal wall } \\
\text { that according } \\
\text { to the health } \\
\text { care personnel } \\
\text { were unlikely } \\
\text { to respond to } \\
\text { physical ther- } \\
\text { apy" was used }\end{array}$ & - & $\begin{array}{l}10 \times 1 \text {-hour ses- } \\
\text { sions of glob- } \\
\text { al therapeutic } \\
\text { Western mas- } \\
\text { sage, includ- } \\
\text { ing effleurage, } \\
\text { petrissage, fric- } \\
\text { tion, tapote- } \\
\text { ment, vibration } \\
\text { and kneading, in } \\
\text { upper and low- } \\
\text { er limbs, trunk, } \\
\text { buttocks, ab- } \\
\text { domen, head } \\
\text { and neck each }\end{array}$ & $\begin{array}{l}\text { No informa- } \\
\text { tion regarding } \\
\text { dose-scaling } \\
\text { or contraindi- } \\
\text { cations. }\end{array}$ \\
\hline
\end{tabular}


Table 1. Description of interventions (Continued)

as an exclusion
criterion. for prescribed

time periods;

participants not

provided with

home exercise

programme.

\begin{tabular}{|c|c|c|c|c|c|c|}
\hline Zeng 2012 & $\begin{array}{l}20,000 \text { ESWT (HB-ESWT-01, } \\
\text { Haibin Medical Equipment Co. } \\
\text { Ltd., China) impulses in } 10 \text { ses- } \\
\text { sions, applied directly to per- } \\
\text { ineal area in which pain was } \\
\text { localised (from anus to scro- } \\
\text { tum); starting energy densi- } \\
\text { ty was } 0.06 \mathrm{~mJ} / \mathrm{mm}^{2} \text { and fre- } \\
\text { quency of } 2 \mathrm{~Hz} \text { was used for all } \\
\text { treatments. }\end{array}$ & $\begin{array}{l}\text { Energy densi- } \\
\text { ty gradually } \\
\text { increased un- } \\
\text { til it reached } \\
\text { the maximum } \\
\text { possible toler- } \\
\text { able pain lev- } \\
\text { el reported by } \\
\text { participant } \\
\text { (recorded and } \\
\text { used in all sub- } \\
\text { sequent ses- } \\
\text { sions). } \\
\text { No information } \\
\text { regarding con- } \\
\text { traindications. }\end{array}$ & 2 weeks. & $\begin{array}{l}\text { Sham ESWT, } \\
\text { which was con- } \\
\text { ducted by set- } \\
\text { ting the ener- } \\
\text { gy level to } 0 \text { (no } \\
\text { shockwave ener- } \\
\text { gy transmission), } \\
\text { under conditions } \\
\text { identical to ac- } \\
\text { tive treatment } \\
\text { group. }\end{array}$ & $\begin{array}{l}\text { No informa- } \\
\text { tion regarding } \\
\text { dose-scaling } \\
\text { or contraindi- } \\
\text { cations. }\end{array}$ & 2 weeks. \\
\hline \multirow[t]{2}{*}{ Gao 2012} & $\begin{array}{l}\text { 60-min treatment with TRFH } \\
\text { (ZRL-II-A cavity intervention } \\
\text { treatment instrument provid- } \\
\text { ed by Shanghai Songhang In- } \\
\text { dustry, Co. Ltd. (Shanghai, Chi- } \\
\text { na), temperature } 40-43^{\circ} \mathrm{C} \text { ) } \\
\text { every day. }\end{array}$ & \multirow[t]{2}{*}{$\begin{array}{l}\text { No information } \\
\text { regarding dose- } \\
\text { scaling or con- } \\
\text { traindications. }\end{array}$} & \multirow[t]{2}{*}{5 days. } & \multirow[t]{2}{*}{$\begin{array}{l}\text { Tamsulosin } 0.2 \\
\text { mg once daily + } \\
\text { clarithromycin } \\
0.25 \text { g bid. }\end{array}$} & \multirow[t]{2}{*}{$\begin{array}{l}\text { No informa- } \\
\text { tion regarding } \\
\text { dose-scaling } \\
\text { or contraindi- } \\
\text { cations. }\end{array}$} & \multirow[t]{2}{*}{6 weeks. } \\
\hline & $\begin{array}{l}\text { TRFH + tamsulosin + clar- } \\
\text { ithromycin ( } 6 \text { weeks). }\end{array}$ & & & & & \\
\hline \multirow[t]{3}{*}{ Yang 2011} & $\begin{array}{l}\text { Biofeedback group, partic- } \\
\text { ipant displayed the elec- } \\
\text { tromyography of pelvic floor } \\
\text { muscle and was instructed to } \\
\text { contract and relax the anus ac- } \\
\text { cording to instructions on dis- } \\
\text { play ( } 20 \text {-min sessions, } 5 \text { times } \\
\text { weekly for } 2 \text { weeks). }\end{array}$ & \multirow[t]{3}{*}{$\begin{array}{l}\text { No information } \\
\text { regarding dose- } \\
\text { scaling or con- } \\
\text { traindications. } \\
\text { All participants } \\
\text { received in- } \\
\text { terventions in } \\
\text { comparison } \\
\text { group. }\end{array}$} & \multirow[t]{3}{*}{2 weeks. } & \multirow{3}{*}{$\begin{array}{l}\text { All participants, } \\
\text { including those } \\
\text { in the "usual } \\
\text { care group" in- } \\
\text { structed to avoid } \\
\text { alcohol and } \\
\text { spicy food, sit- } \\
\text { ting too long and } \\
\text { holding urine, } \\
\text { catching a cold. } \\
\text { Advised to be } \\
\text { physically active } \\
\text { and do exercise, } \\
\text { have sex regu- } \\
\text { larly, have warm } \\
\text { sitz baths. }\end{array}$} & \multirow{3}{*}{$\begin{array}{l}\text { They were in- } \\
\text { structed to } \\
\text { discontinue } \\
\text { antibiotics, } \\
\text { alpha-block- } \\
\text { ers and other } \\
\text { medications } \\
\text { during the tri- } \\
\text { al. } \\
\text { No informa- } \\
\text { tion regarding } \\
\text { dose-scaling } \\
\text { or contraindi- } \\
\text { cations. }\end{array}$} & \multirow[t]{3}{*}{-} \\
\hline & $\begin{array}{l}\text { Electrical stimulation group, } \\
\text { anal electrodes using intensi- } \\
\text { ty of } 6^{\sim} 23 \mathrm{~mA} \text {, for } 10^{\sim} 20 \mathrm{~s} \text { and } \\
\text { relaxation for } 10^{\sim} 20 \mathrm{~s},(20-\mathrm{min} \\
\text { sessions; } 5 \text { times each week } \\
\text { for } 2 \text { weeks). }\end{array}$ & & & & & \\
\hline & $\begin{array}{l}\text { Biofeedback + electrical stimu- } \\
\text { lation (factorial design). }\end{array}$ & & & & & \\
\hline $\begin{array}{l}\text { Zhang } \\
\text { 2011a }\end{array}$ & $\begin{array}{l}\text { Taijiquan (Tai Chi) exercises for } \\
20-40 \text { min every day. All partic- } \\
\text { ipants received herbal thera- } \\
\text { py (no further specifications } \\
\text { available) for } 1 \text { month after. }\end{array}$ & $\begin{array}{l}\text { No information } \\
\text { regarding dose- } \\
\text { scaling or con- } \\
\text { traindications. }\end{array}$ & 1 month. & $\begin{array}{l}\text { All participants } \\
\text { received herbal } \\
\text { therapy (no fur- } \\
\text { ther specifica- } \\
\text { tions available) }\end{array}$ & $\begin{array}{l}\text { No informa- } \\
\text { tion regarding } \\
\text { dose-scaling } \\
\text { or contraindi- } \\
\text { cations. }\end{array}$ & 1 month. \\
\hline
\end{tabular}


Table 1. Description of interventions (Continued)

for 1 month af-

ter.

\begin{tabular}{|c|c|}
\hline $\begin{array}{l}\text { Samhan } \\
2011\end{array}$ & $\begin{array}{l}\text { TENS daily for mean of } 20 \text { min } \\
\text { in painful area, mean frequen- } \\
\text { cy } 100 \mathrm{~Hz} \text {, pulse width } 100 \mu \mathrm{s} \\
\text { and intensity } 25 \mathrm{~mA} \text { daily, } 5 \\
\text { times weekly. }\end{array}$ \\
\hline \multirow[t]{4}{*}{ Lee 2009} & $\begin{array}{l}\text { Advice of hot sitz baths and } \\
\text { exercise and } 12 \times 20 \text {-min ses- } \\
\text { sions of electroacupuncture in } \\
6 \text { weeks: } 6 \text { acupoints at bilater- } \\
\text { al: }\end{array}$ \\
\hline & BL32 zhongliao \\
\hline & BL33 ciliao \\
\hline & $\begin{array}{l}\text { GB30 huantiao; preparation } \\
\text { alcohol pads and disposable } \\
\text { stainless steel needles ( } 400.25 \\
\text { mm, Dongbang Acupuncture, } \\
\text { Chungnam, Republic of Ko- } \\
\text { rea); at GB30, disposable stain- } \\
\text { less steel needles ( } 700.30 \mathrm{~mm} \text {, } \\
\text { Dongbang Acupuncture) were } \\
\text { inserted deeply to reach the } \\
\text { myofascial trigger point of piri- } \\
\text { formis muscle. }\end{array}$ \\
\hline
\end{tabular}

No information regarding dosescaling or contraindications.

1 month.

Same as active
treatment group
but with ma-

chine turned off.

No information
regarding dose-
scaling or con-
traindications.

6 weeks.

Advice and exercise and 12 sessions of sham electroacupuncture, which included the same number and type of needle, duration and frequency of sessions as for the advice and exercise treatment, but treatment was delivered superficially at non-acupoints $15 \mathrm{~mm}$ to the lateral of each corresponding acupoint; points were not stimulated electrically, but sound of pulse generator was heard by the participants.

\begin{tabular}{|c|c|c|c|c|c|c|}
\hline Chen 2009 & $\begin{array}{l}\text { Acupuncture + warm needle } \\
\text { moxibustion. } \\
\text { Acupuncture at acupoints: } \\
\text { BI18 GanYu } \\
\text { BI23 ShenYu } \\
\text { BI54 ZhiBian } \\
\text { BI26 GuanYuan } \\
\text { Ren3 ZhongJi } \\
\text { SP9 YinLingQuan } \\
\text { SP6 SanYinJiao } \\
\text { For } 5 \text { s each; needles were re- } \\
\text { moved afterwards; once daily } \\
\text { for } 1 \text { month. } \\
\text { Acupuncture procedures same } \\
\text { as acupuncture + warm needle } \\
\text { moxibustion. Moxibustion not } \\
\text { performed. Treated once daily. } \\
\text { Course of treatment: } 1 \text { month. }\end{array}$ & $\begin{array}{l}\text { For those treat- } \\
\text { ed with moxi- } \\
\text { bustion: } \\
\text { needles were } \\
\text { left afterwards } \\
\text { and the tails } \\
\text { of which were } \\
\text { then covered } \\
\text { with } 2 \text { cm moxa } \\
\text { sticks. Moxa } \\
\text { sticks were } \\
\text { then ignited. } \\
\text { Repeated mox- } \\
\text { ibustion twice } \\
\text { more for each } \\
\text { acupoint. } \\
\text { No information } \\
\text { regarding dose- } \\
\text { scaling or con- } \\
\text { traindications. }\end{array}$ & 1 month. & $\begin{array}{l}\text { Prostat tablet } \\
\text { orally twice dai- } \\
\text { ly: pollen extract. } \\
\text { Course of treat- } \\
\text { ment; } 1 \text { month. }\end{array}$ & $\begin{array}{l}\text { No informa- } \\
\text { tion regarding } \\
\text { dose-scaling } \\
\text { or contraindi- } \\
\text { cations. }\end{array}$ & 1 month. \\
\hline
\end{tabular}

No informa- 1 month.
tion regarding
dose-scaling
or contraindi-
cations.

No informa- 6 weeks
tion regarding dose-scaling or contraindications. 
Table 1. Description of interventions (Continued)

Zimmer- Perineally applied ESWT weekmann 2009 ly (3000 pulses each; maximum total energy flow density: $0.25 \mathrm{~mJ} / \mathrm{mm}^{2}$; frequency: 3 $\mathrm{Hz}$ ); position of transducer was changed after every 500 pulses to scan prostatic and pelvic floor region (Duolith SD1, Storz Medical, Tägerwilen, Switzerland); penetration depth 35-65 $\mathrm{mm}$.
No information regarding dosescaling or contraindications.
4 weeks.

\section{ment performed} with same therapy head, which was also fitted with a placebo stand-off. Standoff contained shock wave-absorbing material, layer of air and air-filled microspheres.

\begin{tabular}{ll}
\hline Marx 2009 & Osteopathic theory of struc- \\
& tural dysfunction using direct \\
& and indirect techniques of ma- \\
& nipulation; including rectal \\
& manipulation of the prostate \\
& and coccyx; 45-min sessions \\
& for 5 weeks.
\end{tabular}

$\begin{array}{llll}\begin{array}{l}\text { No information } \\ \text { regarding dose- }\end{array} & 5 \text { weeks. } & \text { Sham exercise } & \text { No informa- } \\ \text { scaling or con- } & \text { program includ- } & \text { tion regarding } & \\ \text { traindications. } & \text { ing a warm-up, } & \text { dose-scaling } & \\ & \text { stretching, limb, } & \text { or contraindi- } \\ & \text { breathing and } & \text { cations. } \\ \text { pelvic floor mus- } & \\ \text { cle exercises; } 30 & \\ \text { min sessions in 5 } & \\ & & \\ & & \end{array}$

\begin{tabular}{|c|c|c|c|c|c|c|}
\hline Oh 2009 & 'Radiofrequency.' & (abstract only) & - & $\begin{array}{l}\text { "Placebo thera- } \\
\text { py." }\end{array}$ & (abstract only) & - \\
\hline $\begin{array}{l}\text { Kabay } \\
2009\end{array}$ & $\begin{array}{l}\text { PTNS applied unilaterally with } \\
26 \text {-gauge stainless steel nee- } \\
\text { dles inserted } 5 \mathrm{~cm} \text { cephalad } \\
\text { from medial malleolus and } \\
\text { posterior to edge of tibia with }\end{array}$ & $\begin{array}{l}\text { No information } \\
\text { regarding dose- } \\
\text { scaling or con- } \\
\text { traindications. }\end{array}$ & 12 weeks. & $\begin{array}{l}\text { Same electrode } \\
\text { procedure for } \\
\text { PTNS; however, } \\
\text { stimulator not } \\
\text { connected. }\end{array}$ & $\begin{array}{l}\text { No informa- } \\
\text { tion regarding } \\
\text { dose-scaling } \\
\text { or contraindi- } \\
\text { cations. }\end{array}$ & 12 weeks. \\
\hline
\end{tabular}
ground neutral electrode placed on same leg near arch of foot; both connected to a stimulator at $200 \mu \mathrm{s}$ with pulse rate $20 \mathrm{~Hz}$ (Medtronic Key Point Net, Medtronic), for $30 \mathrm{~min}$.

\begin{tabular}{|c|c|c|c|c|c|c|}
\hline Yoo 2009 & $\begin{array}{l}\text { TRMT alone for } 12 \text { weeks; us- } \\
\text { ing a Uro-DR Device (Somang } \\
\text { Medical; Kangreung, Korea), } \\
\text { at intrarectal temperature of } \\
43^{\circ} \mathrm{C} \text { for } 30 \mathrm{~min} \text {, at a medium } \\
\text { heating rate. }\end{array}$ & $\begin{array}{l}\text { No information } \\
\text { regarding dose- } \\
\text { scaling or con- } \\
\text { traindications. }\end{array}$ & 12 weeks. & $\begin{array}{l}\text { Medical thera- } \\
\text { py: ciprofloxacin } \\
500 \text { mg bid and } \\
\text { NSAIDs } \\
\text { C: TRMT + med- } \\
\text { ical therapy. }\end{array}$ & $\begin{array}{l}\text { No informa- } \\
\text { tion regarding } \\
\text { dose-scaling } \\
\text { or contraindi- } \\
\text { cations. }\end{array}$ & 12 weeks. \\
\hline Lee 2008 & $\begin{array}{l}4 \text { acupoints prepared, then } \\
\text { sterile, disposable stainless } \\
\text { steel needles (Suzhou Huan- } \\
\text { Qiu Acupuncture Medical Sup- } \\
\text { plies, Suzhou, China) placed } \\
\text { perpendicularly in 30-min } \\
\text { sessions twice weekly in acu- } \\
\text { points: } \\
\text { CV1 Guan Yuan } \\
\text { CV4 Huiyin } \\
\text { SP6 Sanyinjiao }\end{array}$ & $\begin{array}{l}\text { No information } \\
\text { regarding dose- } \\
\text { scaling or con- } \\
\text { traindications. }\end{array}$ & 10 weeks. & $\begin{array}{l}\text { Sham acupunc- } \\
\text { ture includ- } \\
\text { ed same num- } \\
\text { ber, duration } \\
\text { and frequen- } \\
\text { cy of sessions } \\
\text { as acupuncture } \\
\text { group at non- } \\
\text { acupoints (15 } \\
\text { mm to left). }\end{array}$ & $\begin{array}{l}\text { No informa- } \\
\text { tion regarding } \\
\text { dose-scaling } \\
\text { or contraindi- } \\
\text { cations. }\end{array}$ & 10 weeks. \\
\hline
\end{tabular}

Non-pharmacological interventions for treating chronic prostatitis/chronic pelvic pain syndrome (Review) 
Table 1. Description of interventions (Continued) SP9 Yinlingquan.

\begin{tabular}{|c|c|c|c|c|c|c|}
\hline Sikiru 2008 & $\begin{array}{l}\text { Antibiotics plus TENS; with } \\
\text { high TENS over painful area, } \\
\text { daily for mean of } 20 \text { min, mean } \\
\text { frequency } 100 \mathrm{~Hz} \text {, pulse width } \\
100 \text { us and intensity } 25 \mathrm{~mA}, 5 \\
\text { times weekly. }\end{array}$ & $\begin{array}{l}\text { Contraindica- } \\
\text { tions: loss of } \\
\text { skin sensation } \\
\text { at and around } \\
\text { painful area, } \\
\text { cardiac pace } \\
\text { maker, previ- } \\
\text { ous exposure to } \\
\text { TENS and other } \\
\text { electroanalge- } \\
\text { sia. }\end{array}$ & 4 weeks. & $\begin{array}{l}\text { Ofloxacin } 300 \text { mg } \\
3 \text { times daily + } \\
\text { placebo tablets. }\end{array}$ & $\begin{array}{l}\text { No informa- } \\
\text { tion regarding } \\
\text { dose-scaling } \\
\text { or contraindi- } \\
\text { cation. }\end{array}$ & 4 weeks. \\
\hline $\begin{array}{l}\text { Giubilei } \\
2007\end{array}$ & $\begin{array}{l}\text { Aerobic exercise group, which } \\
\text { included an } 18 \text {-week walking } \\
\text { programme, } 3 \text { times weekly; } \\
\text { with a warm up and cool-down } \\
\text { regimen, postural muscle and } \\
\text { isometric strengthening ex- } \\
\text { ercises, } 40 \text { min of fast-paced } \\
\text { walking on 'in-outdoor' track } \\
\text { (achieving } 70 / 80 \% \text { of predict- } \\
\text { ed maximum heart rate for } \\
\text { their age). }\end{array}$ & $\begin{array}{l}\text { Participants } \\
\text { with a 'lack of } \\
\text { interest,' 'lack } \\
\text { of time' and } \\
\text { 'lack of confi- } \\
\text { dence' to en- } \\
\text { gage physical } \\
\text { activity exclud- } \\
\text { ed from study. } \\
\text { No information } \\
\text { regarding dose- } \\
\text { scaling. }\end{array}$ & 18 weeks. & $\begin{array}{l}\text { Placebo/flexi- } \\
\text { bility and mo- } \\
\text { tion exercise pro- } \\
\text { gramme; with } \\
\text { same period and } \\
\text { with same fre- } \\
\text { quency of aer- } \\
\text { obic exercise } \\
\text { group, maintain- } \\
\text { ing their heart } \\
\text { rate under } 100 \\
\text { beats per min. }\end{array}$ & $\begin{array}{l}\text { Participants } \\
\text { with a 'lack of } \\
\text { interest,' 'lack } \\
\text { of time' and } \\
\text { 'lack of confi- } \\
\text { dence' to en- } \\
\text { gage physical } \\
\text { activity were } \\
\text { excluded from } \\
\text { the study. } \\
\text { No informa- } \\
\text { tion regarding } \\
\text { dose-scaling. }\end{array}$ & 18 weeks. \\
\hline Paick 2006 & $\begin{array}{l}\text { Extracorporeal magnetic in- } \\
\text { nervation using Neoconrol sys- } \\
\text { tem (Neotonus Inc., Mariet- } \\
\text { ta, GA, USA) that generates a } \\
\text { magnetic field directed in the } \\
\text { seat of chair and concentrated } \\
\text { in region of pelvic muscles; } 2 \\
\text { sessions weekly lasting } 20 \text { min } \\
\text { each. }\end{array}$ & $\begin{array}{l}1 \text { st } 10 \text { min used } \\
10 \mathrm{~Hz} \text { field, } 2 \\
\text { min rest and } \\
\text { then } 10 \text { min of } \\
50 \mathrm{~Hz} \text { field. } \\
\text { No information } \\
\text { regarding con- } \\
\text { traindications. }\end{array}$ & 6 weeks. & $\begin{array}{l}\text { Medical therapy } \\
\text { with terazosin } 4 \\
\text { mg daily. }\end{array}$ & $\begin{array}{l}\text { They start- } \\
\text { ed } 2 \text { mg daily } \\
\text { for } 1 \text { st } 7 \text { days, } \\
\text { and continued } \\
\text { to receive } 4 \\
\text { mg daily for } \\
\text { the following } \\
5 \text { weeks. } \\
\text { No informa- } \\
\text { tion regarding } \\
\text { contraindica- } \\
\text { tions. }\end{array}$ & 6 weeks. \\
\hline Ateya 2006 & $\begin{array}{l}\text { Participants received antibi- } \\
\text { otics empirically and prosta- } \\
\text { tic massage ( } 3 \text { times week- } \\
\text { ly for } 4 \text { weeks). Prostate mas- } \\
\text { saged from above and lateral } \\
\text { to gland, } 6 \text { times on each side, } \\
\text { by gentle and firm pressure } \\
\text { of finger directed downwards } \\
\text { and inwards, followed by a few } \\
\text { strokes in middle from above } \\
\text { downwards. }\end{array}$ & $\begin{array}{l}\text { No information } \\
\text { regarding dose- } \\
\text { scaling or con- } \\
\text { traindications. }\end{array}$ & - & $\begin{array}{l}\text { Empirical an- } \\
\text { tibiotic therapy } \\
\text { alone without } \\
\text { prostatic mas- } \\
\text { sage. }\end{array}$ & $\begin{array}{l}\text { 'In the } 44 \text { par- } \\
\text { ticipants with } \\
\text { negative cul- } \\
\text { tures, antibi- } \\
\text { otic selec- } \\
\text { tion included } \\
\text { quinolones } \\
\text { ( } 28 \text { partici- } \\
\text { pants), clin- } \\
\text { damycin/metron- } \\
\text { idazole (11 } \\
\text { participants), } \\
\text { ampicillin/ } \\
\text { clavulanic } \\
\text { acid ( } 2 \text { partic- } \\
\text { ipants), and } \\
\text { azithromycin } \\
\text { (1 partici- }\end{array}$ & - \\
\hline
\end{tabular}


Table 1. Description of interventions (Continued)

pant). All par-

ticipants con-

tinued the 1

month of

treatment.'

\begin{tabular}{|c|c|c|c|c|c|c|}
\hline Shen 2006 & $\begin{array}{l}\text { Medical therapy with modified } \\
\text { BiXieFenQing Drink in morning } \\
\text { and evening, } 200 \mathrm{~mL} \text { each + } \\
\text { prostate massage once week- } \\
\text { ly. }\end{array}$ & $\begin{array}{l}\text { No information } \\
\text { regarding dose- } \\
\text { scaling or con- } \\
\text { traindications. }\end{array}$ & - & $\begin{array}{l}\text { Medical therapy } \\
\text { alone. }\end{array}$ & $\begin{array}{l}\text { No informa- } \\
\text { tion regarding } \\
\text { dose-scaling } \\
\text { or contraindi- } \\
\text { cations. }\end{array}$ & - \\
\hline Rowe 2005 & $\begin{array}{l}\text { Participant seated in a } \\
\text { Neotonus Electromagnetic } \\
\text { Chair (Neotonus Inc., Marietta, } \\
\text { GA, USA), for } 2 \text { consecutive 15- } \\
\text { min periods; } 2 \text { sessions week- } \\
\text { ly. }\end{array}$ & $\begin{array}{l}\text { 1st period } 10 \\
\mathrm{~Hz}, 2 \text { nd period } \\
50 \mathrm{~Hz} \text {. } \\
\text { No information } \\
\text { regarding con- } \\
\text { traindications. }\end{array}$ & 4 weeks. & $\begin{array}{l}\text { Participants } \\
\text { seated in chair, } \\
\text { ventilation } \\
\text { mechanism acti- } \\
\text { vated, but no ac- } \\
\text { tive stimulation } \\
\text { applied. }\end{array}$ & $\begin{array}{l}\text { No informa- } \\
\text { tion regarding } \\
\text { dose-scaling } \\
\text { or contraindi- } \\
\text { cations. }\end{array}$ & 4 weeks. \\
\hline Fang 2005 & $\begin{array}{l}\text { He-Ne laser directed to } \\
\text { prostate. Optic fibre was in- } \\
\text { serted from urethra and locat- } \\
\text { ed at prostatic urethra; } 10 \mathrm{~mW} \\
\text { output at } 18 \mathrm{~J} \text { each time. }\end{array}$ & $\begin{array}{l}1 \text { course of } \\
\text { treatment: } 10 \\
\text { doses of radia- } \\
\text { tion ( } 2 \text { sessions } \\
\text { weekly). } \\
\text { Participants } \\
\text { discontinued } \\
\text { all other treat- } \\
\text { ments, except } \\
\text { for some of the } \\
\text { participants, } \\
\text { short-term sul- } \\
\text { fa-drugs were } \\
\text { administered } \\
\text { temporarily to } \\
\text { prevent infec- } \\
\text { tion. }\end{array}$ & - & $\begin{array}{l}\text { 'Routine care' } \\
\text { for prostatitis in- } \\
\text { cluding antibi- } \\
\text { otics, pollen ex- } \\
\text { tract, Chinese } \\
\text { medicine, physi- } \\
\text { cal therapy and } \\
\text { lifestyle interven- } \\
\text { tions. }\end{array}$ & $\begin{array}{l}\text { Sulfamethox- } \\
\text { azole tablets, } \\
2 \text { tablets, } \\
\text { orally bid, } 60 \\
\text { days of fluo- } \\
\text { roquinolones } \\
\text { such as lev- } \\
\text { ofloxacin } \\
0.2 \text { g, bid, } 14 \\
\text { days of pollen } \\
\text { drugs such as } \\
\text { Prostat; Chi- } \\
\text { nese patent } \\
\text { drugs such } \\
\text { as salviano- } \\
\text { lic acid B and } \\
\text { saponins of } \\
\text { panax noto- } \\
\text { ginseng mix- } \\
\text { ture (SalB/ } \\
\text { PNS); hot wa- } \\
\text { ter bath and } \\
\text { advise on } \\
\text { lifestyle. }\end{array}$ & - \\
\hline $\begin{array}{l}\text { Kastner } \\
2004\end{array}$ & $\begin{array}{l}\text { Transurethral microwave ther- } \\
\text { motherapy through catheter } \\
\text { connected to a Targis System } \\
\text { estimated peak intraprostatic } \\
\text { temperatures of } 55^{\circ} \mathrm{C} \text {. }\end{array}$ & $\begin{array}{l}\text { No information } \\
\text { regarding dose- } \\
\text { scaling or con- } \\
\text { traindications. }\end{array}$ & - & $\begin{array}{l}\text { Transurethral mi- } \\
\text { crowave ther- } \\
\text { motherapy } \\
\text { through catheter } \\
\text { connected to a } \\
\text { Targis System } \\
\text { estimated peak } \\
\text { intraprostatic } \\
\text { temperatures of } \\
70^{\circ} \mathrm{C} \text {. }\end{array}$ & $\begin{array}{l}\text { No informa- } \\
\text { tion regarding } \\
\text { dose-scaling } \\
\text { or contraindi- } \\
\text { cations. }\end{array}$ & - \\
\hline Wang 2002 & $\begin{array}{l}\text { External radiofrequency hy- } \\
\text { perthermia. }\end{array}$ & $\begin{array}{l}\text { Applied exter- } \\
\text { nally: } 2 \text { elec- } \\
\text { trodes placed } \\
\text { at hip and low- }\end{array}$ & $1^{\sim} 2$ weeks. & $\begin{array}{l}\text { Same external } \\
\text { radiofrequency } \\
\text { hyperthermia + } \\
\text { terazosin } 2 \mathrm{mg} \text {. }\end{array}$ & $\begin{array}{l}\text { Daily for } 2 \\
\text { days and then } \\
\text { dose was in- } \\
\text { creased to } 2\end{array}$ & 12 weeks. \\
\hline
\end{tabular}

Non-pharmacological interventions for treating chronic prostatitis/chronic pelvic pain syndrome (Review) Copyright $\odot 2018$ The Cochrane Collaboration. Published by John Wiley \& Sons, Ltd. 
Table 1. Description of interventions (Continued)

$$
\begin{aligned}
& \text { er abdomen, } \\
& 5^{\sim} 7 \mathrm{~cm} \text { away } \\
& \text { from skin, with } \\
& \text { pubic symph- } \\
& \text { ysis as centre } \\
& \left(42.5^{\sim} 43.5^{\circ} \mathrm{C}\right) \\
& 1^{\sim} 2 \text { hours each } \\
& \text { time, course of } \\
& \text { treatment: } 2^{\sim} 3 \\
& \text { times, interval: } \\
& 1^{\sim} 2 \text { weeks. }
\end{aligned}
$$

No information regarding dosescaling or contraindications. mg bid for 12 weeks.

No information regarding contraindications for terazosin.

\begin{tabular}{ll}
\hline Leskinen & TUNA using 465-kHz radiofre- \\
2002 & quency energy and formal \\
& needle insertion technique as \\
& described by Issa 1996; treat- \\
& ment applied on 2 planes on \\
& both lateral lobes of prostate \\
& in all participants so target \\
& temperature of $50^{\circ} \mathrm{C}$ at needle \\
& tip was achieved for $\geq 1$ min; \\
& under spinal analgesia and an- \\
& tibiotic prophylaxis.
\end{tabular}

No information regarding dosescaling or contraindications.

3 sham ure-
throscopy per-
formed so it was
seemingly iden-
tical to TUNA
in participant's
opinion; under
spinal analgesia
and antibiotic
prophylaxis.

No information regarding dose-scaling or contraindi-

\begin{tabular}{|c|c|c|c|c|c|c|}
\hline $\begin{array}{l}\text { Nickel } \\
1996\end{array}$ & $\begin{array}{l}\text { Transurethral microwave ther- } \\
\text { motherapy consisted of } 1 \times 1- \\
\text { hour treatment with comput- } \\
\text { er-driven device that elevat- } \\
\text { ed prostate interstitial tem- } \\
\text { peratures to } 45-60^{\circ} \mathrm{C} \text {, a range } \\
\text { that does not cause significant } \\
\text { necrosis of normal prostatic } \\
\text { tissue. }\end{array}$ & $\begin{array}{l}\text { No information } \\
\text { regarding dose- } \\
\text { scaling or con- } \\
\text { traindications. }\end{array}$ & $\begin{array}{l}\text { Single ses- } \\
\text { sion. }\end{array}$ & $\begin{array}{l}\text { Sham therapy } \\
\text { consisted of } 1 \times \\
\text { 1-hour session } \\
\text { with same device } \\
\text { using sham soft- } \\
\text { ware. }\end{array}$ & $\begin{array}{l}\text { No informa- } \\
\text { tion regarding } \\
\text { dose-scaling } \\
\text { or contraindi- } \\
\text { cations. }\end{array}$ & $\begin{array}{l}\text { Single ses- } \\
\text { sion. }\end{array}$ \\
\hline $\begin{array}{l}\text { Muraro } \\
1995\end{array}$ & $\begin{array}{l}\text { Seaprose S (Flaminase, For- } \\
\text { menti) } 30 \mathrm{mg} 3 \text { times daily in } \\
\text { combination with local hyper- } \\
\text { thermia, total of } 7 \text { sessions on } \\
\text { alternate days of } 60 \text {-min dura- } \\
\text { tion, reaching local tempera- } \\
\text { ture of } 42.5-43.5^{\circ} \mathrm{C} \text {. }\end{array}$ & $\begin{array}{l}\text { No information } \\
\text { regarding dose- } \\
\text { scaling. } \\
\text { Contraindica- } \\
\text { tion to those } \\
\text { with hypersen- } \\
\text { sitivity to the } \\
\text { drug. }\end{array}$ & - & $\begin{array}{l}7 \text { sessions of lo- } \\
\text { cal hyperthermia } \\
\text { alone. }\end{array}$ & $\begin{array}{l}\text { No informa- } \\
\text { tion regarding } \\
\text { dose-scaling } \\
\text { or contraindi- } \\
\text { cations. }\end{array}$ & - \\
\hline
\end{tabular}
cations.

Vassily 2 TRMT 6 sessions over 2 weeks
1999 each.

No information
regarding dose-
scaling or con-
traindications.

\author{
Sham procedure. No informa- \\ tion regarding \\ dose-scaling \\ or contraindi- \\ cations.
}

Montorsi I1: 1 session of transrectal hyperthermia weekly for 4 weeks.

12: 1 session of transrectal hyperthermia weekly for 6 weeks.

13: 2 sessions of transrectal hyperthermia weekly for 3 weeks. 
Table 1. Description of interventions (Continued)

Prostathermer 99D system (Biodan Ltd, Rehovot, Israel), target temperature reached within 1st 10 min of treatment, microwaves at $915 \mathrm{MHz}$, thermosensors for monitoring rectal temperature, cooling system for anterior rectal wall, thermosensors for urethra temperatures, outpatient basis, required local anaesthesia, with $2 \%$ xylocaine jelly before insertion of the catheter.

\begin{tabular}{lllll}
\hline Shah 1993 & $\begin{array}{l}\text { TRMT, } 4 \times 1 \text {-hour treatment } \\
\text { over } 2 \text { or } 3 \text { weeks. Tempera- } \\
\text { ture raised to } 43.8{ }^{\circ} \mathrm{C} \text { with in- } \\
\text { put of } 40 \mathrm{~W} .\end{array}$ & $\begin{array}{l}\text { No information } \\
\text { regarding dose- } \\
\text { scaling or con- } \\
\text { traindications. } \\
\text { (abstract only) }\end{array}$ & $\begin{array}{l}2^{\sim} 3 \text { weeks } \\
\text { temperature }<37\end{array}$ & $\begin{array}{l}\text { Sham group with } \\
\text { tion regarding } \\
\text { dose-scaling } \\
\text { or contraindi- } \\
\text { cations. }\end{array}$ \\
& & & (abstract only) \\
\hline
\end{tabular}

- denotes not reported.

bid: twice daily; C: comparator; ESWT: extracorporeal shockwave therapy; I: intervention; min: minute; NSAID: non-steroidal antiinflammatory drug; PTNS: posterior tibial nerve stimulation; s: second; TENS: transcutaneous electrical nerve stimulation; TRFH: transrectal radiofrequency hyperthermia; TRMT: transrectal microwave thermotherapy; TUNA: transurethral needle ablation. 


\begin{tabular}{|c|c|c|c|c|c|c|c|c|c|}
\hline Study ID & $\begin{array}{l}\text { Intervention(s) and com- } \\
\text { parator(s) }\end{array}$ & $\begin{array}{l}\text { Ran- } \\
\text { domised } \\
\text { (n) }\end{array}$ & $\begin{array}{l}\text { Analysed } \\
\text { (n) }\end{array}$ & $\begin{array}{l}\text { Duration of } \\
\text { interven- } \\
\text { tion and fol- } \\
\text { low-up }\end{array}$ & $\begin{array}{l}\text { Description of partic- } \\
\text { ipants } a\end{array}$ & $\begin{array}{l}\text { Baseline } \\
\text { NIH-CPSIb } \\
\text { score }\end{array}$ & $\begin{array}{l}\text { Trial peri- } \\
\text { od (year } \\
\text { to year) }\end{array}$ & Country & Setting \\
\hline \multirow[t]{2}{*}{$\begin{array}{l}\text { Neimark } \\
2016\end{array}$} & $\begin{array}{l}\text { I: hypercapnic hypoxia + med- } \\
\text { ical therapy }\end{array}$ & 17 & 17 & \multirow{2}{*}{$\begin{array}{l}\text { D: } 10 \text { days } \\
\text { F: } 10 \text { days }\end{array}$} & \multirow{2}{*}{$\begin{array}{l}\text { Age: mean } 37 \\
\text { No specified previous } \\
\text { treatment }\end{array}$} & \multirow[t]{2}{*}{$\mathrm{N} / \mathrm{A}$} & \multirow[t]{2}{*}{$\mathrm{N} / \mathrm{A}$} & \multirow[t]{2}{*}{ Russia } & \multirow[t]{2}{*}{$\begin{array}{l}\text { Outpa- } \\
\text { tient }\end{array}$} \\
\hline & C: medical therapy & 20 & 20 & & & & & & \\
\hline \multirow[t]{2}{*}{$\begin{array}{l}\text { Pajovic } \\
2016\end{array}$} & $\begin{array}{l}\text { I: extracorporeal shockwave } \\
\text { therapy + medical care }\end{array}$ & 30 & 30 & \multirow{2}{*}{$\begin{array}{l}\text { D: } 12 \text { weeks } \\
\text { F: } 24 \text { weeks }\end{array}$} & \multirow{2}{*}{$\begin{array}{l}\text { Age: mean } 39.4 \\
\text { No specified previous } \\
\text { treatment }\end{array}$} & 29.3 & \multirow[t]{2}{*}{$2013-2015$} & \multirow[t]{2}{*}{$\begin{array}{l}\text { Montene- } \\
\text { gro }\end{array}$} & \multirow[t]{2}{*}{$\begin{array}{l}\text { Outpa- } \\
\text { tient }\end{array}$} \\
\hline & C: medical care & 30 & 30 & & & 31.06 & & & \\
\hline \multirow{2}{*}{$\begin{array}{l}\text { Kucuk } \\
2015\end{array}$} & I: acupuncture & 28 & 28 & \multirow{2}{*}{$\begin{array}{l}\text { D: } 7 \text { weeks } \\
\text { F: } 10 \text { weeks }\end{array}$} & \multirow{2}{*}{$\begin{array}{l}\text { Age: mean } 33 \\
\text { No previous treatment }\end{array}$} & 20.36 & \multirow[t]{2}{*}{ 2008-2009 } & \multirow[t]{2}{*}{ Turkey } & \multirow{2}{*}{$\begin{array}{l}\text { Outpa- } \\
\text { tient }\end{array}$} \\
\hline & C: medical care & 26 & 26 & & & 22.92 & & & \\
\hline \multirow{2}{*}{$\begin{array}{l}\text { Sahin } \\
2015\end{array}$} & I: acupuncture & 50 & 45 & \multirow{2}{*}{$\begin{array}{l}\text { D: } 6 \text { weeks } \\
\text { F: } 24 \text { weeks }\end{array}$} & \multirow{2}{*}{$\begin{array}{l}\text { Age: } 20-50 \\
\text { All had received med- } \\
\text { ical therapy }\end{array}$} & 26.5 & \multirow{2}{*}{$\begin{array}{l}\text { Not avail- } \\
\text { able }\end{array}$} & \multirow[t]{2}{*}{ Turkey } & \multirow{2}{*}{$\begin{array}{l}\text { Outpa- } \\
\text { tient }\end{array}$} \\
\hline & C: sham acupuncture & 50 & 46 & & & 27.0 & & & \\
\hline \multirow[t]{2}{*}{ Zhao 2015} & I: early circumcision & 384 & 358 & \multirow{2}{*}{$\begin{array}{l}\text { D: } 3 \text { months } \\
\text { F: } 3 \text { months }\end{array}$} & \multirow{2}{*}{$\begin{array}{l}\text { Age: mean } 33 \\
\text { No specified previous } \\
\text { treatment }\end{array}$} & 21 & \multirow[t]{2}{*}{$2013-2014$} & \multirow[t]{2}{*}{ China } & \multirow{2}{*}{$\begin{array}{l}\text { Outpa- } \\
\text { tient }\end{array}$} \\
\hline & C: delayed circumcision & 390 & 355 & & & 21 & & & \\
\hline \multirow{2}{*}{$\begin{array}{l}\text { Gallo } \\
2014\end{array}$} & I: lifestyle interventions & 50 & 39 & \multirow{2}{*}{$\begin{array}{l}\text { D: } 3 \text { months } \\
\text { F: } 3 \text { months }\end{array}$} & \multirow{2}{*}{$\begin{array}{l}\text { Age: mean 33-34 } \\
\text { No specified previous } \\
\text { treatment }\end{array}$} & 22.1 & \multirow[t]{2}{*}{$2012-2013$} & \multirow[t]{2}{*}{ Italy } & Outpa- \\
\hline & C: no intervention & 50 & 50 & & & 21.9 & & & \\
\hline $\begin{array}{l}\text { Kessler } \\
2014\end{array}$ & $\begin{array}{l}\text { I: sono-electromagnetic ther- } \\
\text { apy }\end{array}$ & 30 & 30 & D: 12 weeks & Age: mean 44-49 & 25.1 & $2009-2012$ & $\begin{array}{l}\text { Switzer- } \\
\text { land }\end{array}$ & $\begin{array}{l}\text { Outpa- } \\
\text { tient }\end{array}$ \\
\hline & C: placebo device & 30 & 30 & & ical therapy & 25.2 & & & \\
\hline Kaikai & I: ultrasound & 35 & 35 & D: 1 month & Age 18-55 & 25.9 & $2013-2014$ & China & Outpa- \\
\hline & $\mathrm{C} 1$ : medical therapy & 35 & 35 & F: 1 month & $\begin{array}{l}\text { All had received med- } \\
\text { ical therapy }\end{array}$ & 26.17 & & & \\
\hline
\end{tabular}




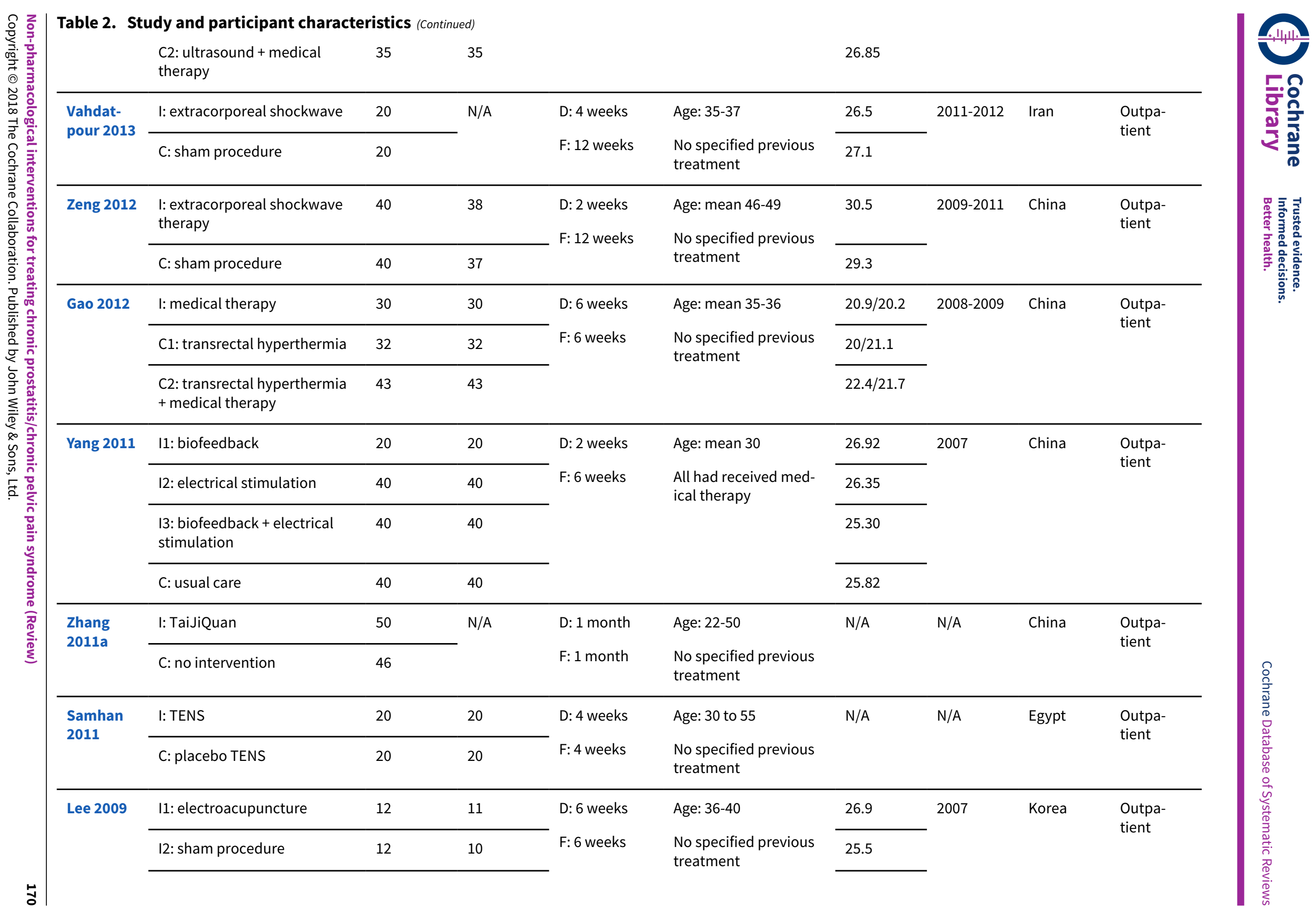




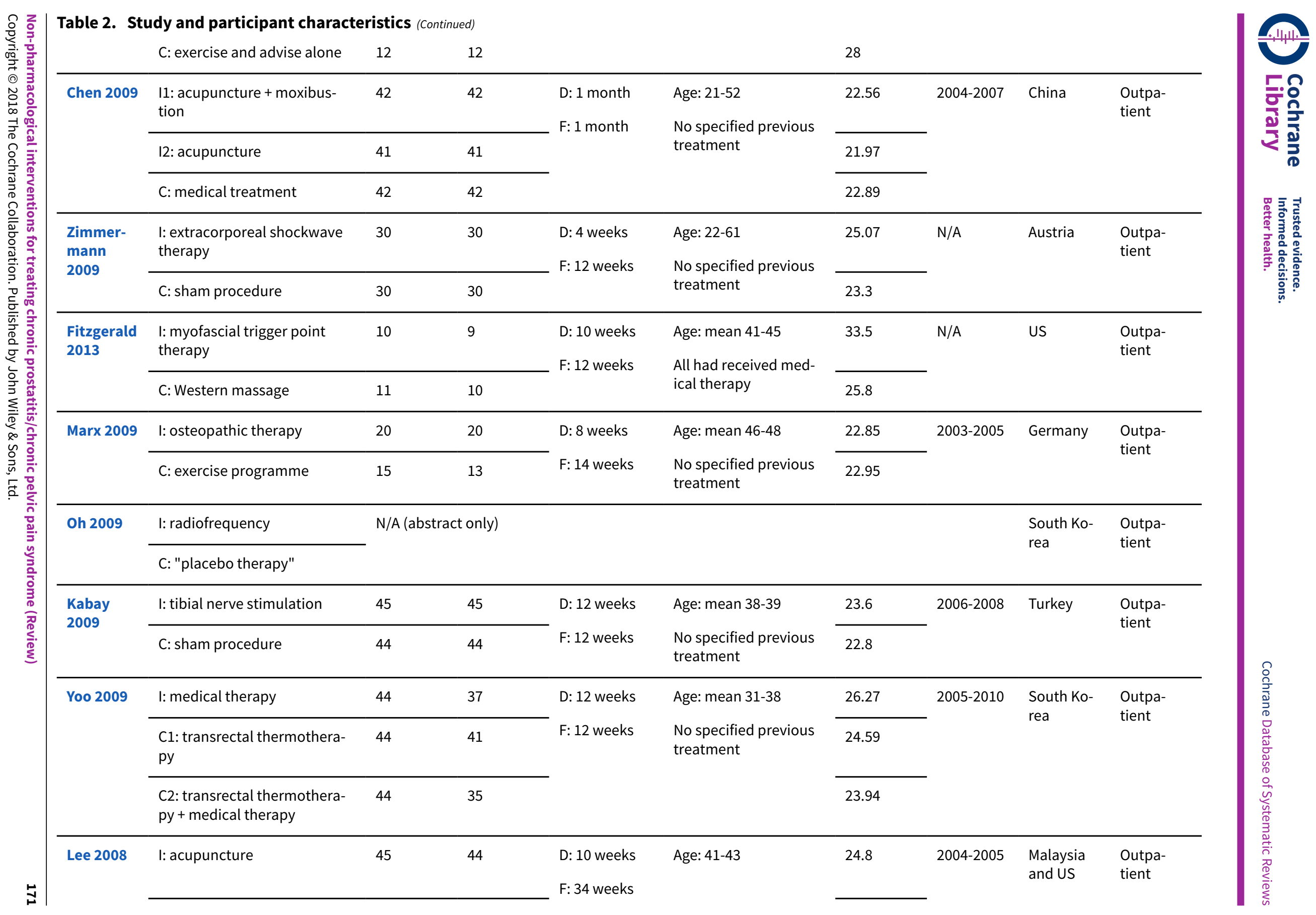




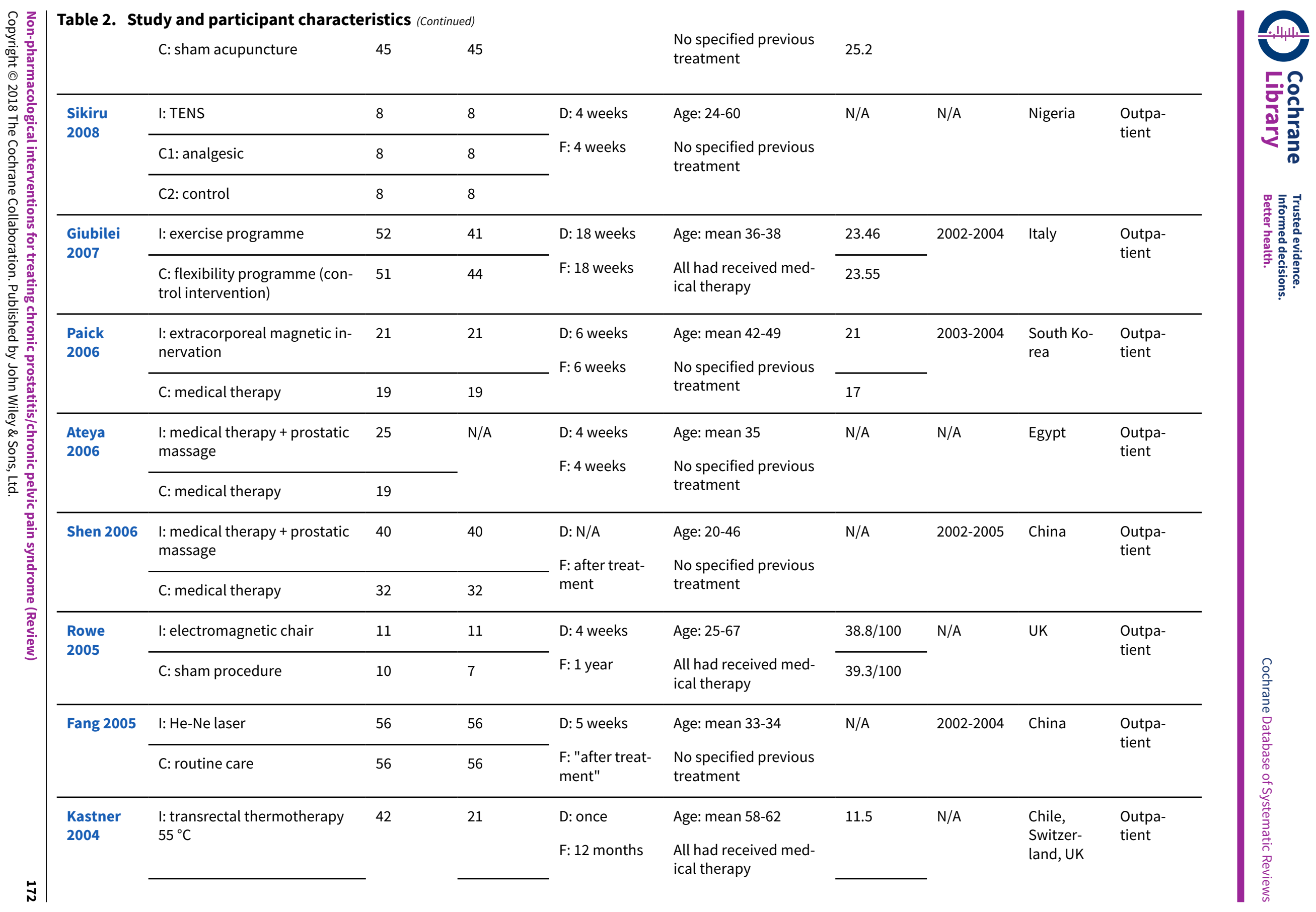




\begin{tabular}{|c|c|c|c|c|c|c|c|c|c|}
\hline & $\begin{array}{l}\text { C: transrectal thermotherapy } \\
70^{\circ} \mathrm{C}\end{array}$ & & 18 & & & 10.9 & & & \\
\hline \multirow[t]{2}{*}{$\begin{array}{l}\text { Wang } \\
2002\end{array}$} & $\begin{array}{l}\text { I: radiofrequency hyperther- } \\
\text { mia }\end{array}$ & 76 & 76 & \multirow{2}{*}{$\begin{array}{l}\text { D: } 12 \text { weeks } \\
\text { F: } 12 \text { weeks }\end{array}$} & \multirow{2}{*}{$\begin{array}{l}\text { Age: mean } 34.2 \\
\text { Not specified previous } \\
\text { treatment }\end{array}$} & \multirow[t]{2}{*}{ N/A } & \multirow[t]{2}{*}{$1998-2001$} & \multirow[t]{2}{*}{ China } & \multirow[t]{2}{*}{$\begin{array}{l}\text { Outpa- } \\
\text { tient }\end{array}$} \\
\hline & $\begin{array}{l}\text { C: radiofrequency hyperther- } \\
\text { mia + terazosin }\end{array}$ & 90 & 88 & & & & & & \\
\hline \multirow[t]{2}{*}{$\begin{array}{l}\text { Leskinen } \\
2002\end{array}$} & $\begin{array}{l}\text { I: transurethral needle abla- } \\
\text { tion }\end{array}$ & 25 & 25 & \multirow{2}{*}{$\begin{array}{l}\text { D: once } \\
\text { F: } 12 \text { months }\end{array}$} & \multirow{2}{*}{$\begin{array}{l}\text { Age: mean } 43-50 \\
\text { All had received med- } \\
\text { ical therapy }\end{array}$} & 37.3 & \multirow[t]{2}{*}{$1998-2001$} & \multirow[t]{2}{*}{ Finland } & \multirow[t]{2}{*}{$\begin{array}{l}\text { Outpa- } \\
\text { tient }\end{array}$} \\
\hline & C: sham procedure & 8 & 8 & & & 33.6 & & & \\
\hline \multirow{2}{*}{$\begin{array}{l}\text { Vassily } \\
1999\end{array}$} & I: transrectal thermotherapy & 80 & $\mathrm{~N} / \mathrm{A}$ & \multirow{2}{*}{$\begin{array}{l}\text { D: } 2 \text { weeks } \\
\text { F: } 6 \text { months }\end{array}$} & \multirow[t]{2}{*}{ N/A (abstract only) } & & & \multirow[t]{2}{*}{ Russia } & \multirow{2}{*}{$\begin{array}{l}\text { Outpa- } \\
\text { tient }\end{array}$} \\
\hline & C: sham procedure & 20 & & & & & & & \\
\hline \multirow[t]{2}{*}{$\begin{array}{l}\text { Nickel } \\
1996\end{array}$} & $\begin{array}{l}\text { I: transurethral thermothera- } \\
\text { py }\end{array}$ & 10 & 10 & \multirow{2}{*}{$\begin{array}{l}\text { D: } 1 \text { session } \\
\text { F: } 3 \text { months }\end{array}$} & \multirow{2}{*}{$\begin{array}{l}\text { Age: } 45-46 \\
\text { All had received med- } \\
\text { ical therapy }\end{array}$} & \multirow[t]{2}{*}{$\mathrm{N} / \mathrm{A}$} & \multirow[t]{2}{*}{$\mathrm{N} / \mathrm{A}$} & \multirow[t]{2}{*}{ Canada } & \multirow[t]{2}{*}{$\begin{array}{l}\text { Outpa- } \\
\text { tient }\end{array}$} \\
\hline & C: sham procedure & 10 & 10 & & & & & & \\
\hline \multirow[t]{2}{*}{$\begin{array}{l}\text { Muraro } \\
1995\end{array}$} & $\begin{array}{l}\text { I: medical therapy plus local } \\
\text { hyperthermia }\end{array}$ & 10 & $\mathrm{~N} / \mathrm{A}$ & \multirow{2}{*}{$\begin{array}{l}\text { D: } 15 \text { days } \\
\text { F: } 4 \text { weeks }\end{array}$} & \multirow{2}{*}{$\begin{array}{l}\text { Age: mean } 42.5 \\
\text { Not specified previous } \\
\text { treatment }\end{array}$} & \multirow[t]{2}{*}{$\mathrm{N} / \mathrm{A}$} & \multirow[t]{2}{*}{$\mathrm{N} / \mathrm{A}$} & \multirow[t]{2}{*}{ Italy } & \multirow[t]{2}{*}{$\begin{array}{l}\text { Outpa- } \\
\text { tient }\end{array}$} \\
\hline & C: local hyperthermia alone & 10 & & & & & & & \\
\hline \multirow{2}{*}{$\begin{array}{l}\text { Montorsi } \\
1993\end{array}$} & \multirow{2}{*}{$\begin{array}{l}\text { Transrectal hyperthermia in } \\
\text { different regimens }\end{array}$} & \multirow[t]{2}{*}{54} & \multirow[t]{2}{*}{54} & D: 3 to 6 & Age: $21-45$ & $\mathrm{~N} / \mathrm{A}$ & 1987-1991 & Italy & Outpa- \\
\hline & & & & F: 26 months & $\begin{array}{l}\text { All had received med- } \\
\text { ical therapy }\end{array}$ & & & & \\
\hline Shah 1993 & I: radiofrequency & 15 & $\mathrm{~N} / \mathrm{A}$ & D: 2-3 weeks & $\mathrm{N} / \mathrm{A}$ & & & UK & Outpa- \\
\hline & C: sham procedure & 15 & & F: 3 months & (abstract only) & & & & \\
\hline $\begin{array}{l}\text { C: comparato } \\
\text { of Health - Ch } \\
{ }^{a} \text { Age: measur } \\
\text { b NIH-CPSI: sc }\end{array}$ & $\begin{array}{l}\text {; D: duration of intervention; F: } \\
\text { onic Prostatitis Symptom Index } \\
\text { ement is in years. } \\
\text { ores were mean or median valu }\end{array}$ & & $\begin{array}{l}\text {-up } \\
\text { ineo }\end{array}$ & $\begin{array}{l}\text { represent the } \\
\text { nerve stimula }\end{array}$ & $\begin{array}{l}\text { ported time points); I: int } \\
\text { on. }\end{array}$ & & ot availabl & & \\
\hline
\end{tabular}




\section{AP PEN DICES}

\section{Appendix 1. CENTRAL search strategy}

\#1 [mh prostatitis]

\#2 prostatit*:ti,ab

\#3 prostatodyn*:ti,ab

\#4 \#1 or \#2 or \#3

\#5 [mh "pelvic pain"]

\#6 pelvic near/3 pain:ti,ab

\#7 CPP*:ti,ab

\#8 \#5 or \#6 or \#7

\#9 \#4 and \#8

\#10 [mh "asymptomatic diseases"]

\#11 asymptom*:ti,ab

\#12 nonsymptom*:ti,ab

\#13 non near/3 symptom*:ti,ab

\#14 abacterial*:ti,ab

\#15 nonbacterial ${ }^{*}:$ ti,ab

\#16 non near/3 bacterial* $:$ ti,ab

\#17 aseptic*:ti,ab

\#18 nonseptic*:ti,ab

\#19 non near/3 septic*:ti,ab

$\# 20 \# 10$ or \#11 or \#12 or \#13 or \#14 or \#15 or \#16 or \#17 or \#18 or \#19

$\# 21 \# 4$ and \#20

\#22 prostatit $^{\star}$ and (pelvic near/3 pain or $\mathrm{CP}^{\mathrm{CPP}}{ }^{\star}$ )

\#23 \#9 or \#21 or \#22

\section{Appendix 2. PubMed search strategy}

\#1 "Prostatitis"[Mesh]

\#2 prostatit* ${ }^{\star}[$ tiab]

\#3 prostatodyn ${ }^{\star}[$ tiab]

\#4 \#1 OR \#2 OR \#3

\#5 “Pelvic Pain"[Mesh]

\#6 pelvic pain[tiab]

$\# 7$ CPP*[tiab]

\#8 \#5 OR \#6 OR \#7 
\#9 \#4 AND \#8

\#10 "Asymptomatic Diseases"[Mesh]

\#11 asymptom ${ }^{\star}[$ tiab]

\#12 nonsymptom ${ }^{\star}[$ tiab]

\#13 non symptom[tiab]

$\# 14$ abacterial ${ }^{\star}[$ tiab]

\#15 nonbacterial* ${ }^{*}$ tiab]

\#16 non bacterial* ${ }^{*}$ tiab]

\#17 aseptic $^{\star}[$ tiab]

$\# 18$ nonseptic ${ }^{\star}[$ tiab]

\#19 non septic*[tiab]

\#20 \#10 OR \#11 OR \#12 OR \#13 OR \#14 OR \#15 OR \#16 OR \#17 OR \#18 OR \#19

\#21 \#4 AND \#20

\#22 chronic prostatitis chronic pelvic pain syndrome[tiab] OR CP CPP* [tiab]

\#23 \#9 OR \#21 OR \#22

\#24 "Randomized Controlled Trial"[Publication Type]

\#25 "Controlled Clinical Trial"[Publication Type]

\#26 randomized[tiab]

\#27 placebo[tiab]

\#28 "drug therapy"[Subheading]

\#29 randomly[tiab]

\#30 trial[tiab]

\#31 groups[tiab]

\#32 \#24 OR \#25 OR \#26 OR \#27 OR \#28 OR \#29 OR \#30 OR \#31

\#33 "Animals"[Mesh] NOT "Humans"[Mesh]

\#34 \#32 NOT \#33

\#35 \#23 AND \#34

\section{Appendix 3. Embase search strategy}

\#1 "prostatitis"/exp

\#2 prostatit*:ab,ti

\#3 prostatodyn*:ab,ti

\#4 \#1 OR \#2 OR \#3

\#5 “pelvic pain"/exp

\#6 $\mathrm{cpp}^{\star}: \mathrm{ab}, \mathrm{ti}$

\#7 (pelvi ${ }^{\star}$ NEAR/3 pain):ab,ti 
\#8 \#5 OR \#6 OR \#7

\#9 \#4 AND \#8

\#10 “asymptomatic disease"/exp

\#11 asymptom*:ab,ti

\#12 nonsymptom*:ab,ti

\#13 (non NEAR/3 symptom*):ab,ti

\#14 abacterial*:ab,ti

\#15 nonbacterial*:ab,ti

\#16 (non NEAR/3 bacterial*):ab,ti

\#17 aseptic*:ab,ti

\#18 nonseptic*:ab,ti

\#19 (non NEAR/3 septic*):ab,ti

\#20 \#10 OR \#11 OR \#12 OR \#13 OR \#14 OR \#15 OR \#16 OR \#17 OR \#18

OR \#19

\#21 \#4 AND \#20

\#22 chronic:ab,ti AND (prostatitis NEAR/3 pelvi ${ }^{\star}$ NEAR/3 pain):ab,ti

\#23 \#9 OR \#21 OR \#22

\#24 random*:ab,ti

\#25 “clinical trial*”

\#26 “health care quality"/exp

\#27 \#24 OR \#25 OR \#26

\#28 \#23 AND \#27

\section{Appendix 4. PsycINFO search strategy}

1 prostatitis.mp.

2 prostatit* $. a b, t i$.

3 prostatodyn*.ab,ti

41 or 2 or 3

5 pelvic pain.mp.

6 (pelvi* adj3 pain).mp.

7 cpp $^{\star}$.ab,ti.

85 or 6 or 7

94 and 8

10 asymptom*.ab,ti.

11 nonsymptom.ab,ti.

12 (non adj3 symptom*).ab,ti. 
13 abacterial $^{*}$.ab,ti.

14 nonbacterial $^{\star}$.ab,ti.

15 (non adj3 bacterial $\left.^{\star}\right)$.ab,ti.

16 aseptic ${ }^{\star} . a b, t i$.

17 nonseptic ${ }^{\star} \cdot a b, t i$.

18 (non adj3 septic*).ab,ti.

1910 or 12 or 13 or 14 or 15 or 16 or 17 or 18

204 and 19

21 (chronic prostatitis adj3 pelvi adj3 pain).ab,ti.

229 or 20 or 21

\section{Appendix 5. CINAHL search strategy}

S1 MH "Prostatitis"

S2 TI prostatit* OR AB prostatit*

S3 TI prostatodyn* OR AB prostatodyn*

\section{S4 S1 OR S2 OR S3}

S5 MH "Pelvic Pain"

S6 TI pelvic pain OR AB pelvic pain

S7 TI cpp* OR AB cpp*

S8 S5 OR S6 OR S7

S9 S4 AND S8

S10 TI asymptom* OR AB asymptom*

S11 TI nonsymptom* OR AB nonsymptom*

S12 TI non N3 symptom* OR AB non N3 symptom*

S13 $\mathrm{Tl}_{\text {abacterial }}{ }^{*}$ OR AB abacterial ${ }^{*}$

S14 TI nonbacterial ${ }^{*}$ OR AB nonbacterial *

S15 TI non N3 bacterial * OR AB non N3 bacterial *

S16 TI aseptic* OR AB aseptic*

S17 TI nonseptic* OR AB nonseptic*

S18 TI non N3 septic* OR AB non N3 septic*

S19 S10 OR S11 OR S12 OR S13 OR S14 OR S15 OR S16 OR S17 OR S18

\section{S20 S4 AND S19}

S21 TI chronic prostatitis/chronic pelvic pain syndrome OR chronic prostatitis

OR AB chronic prostatitis/chronic pelvic pain syndrome OR TI chronic prostatitis chronic pelvic pain syndrome OR chronic prostatitis OR AB chronic prostatitis chronic pelvic pain syndrome 
S22 S9 OR S20 OR S21

\section{Appendix 6. ClinicalTrials.gov/Controlled Trials/WHO ICTRP search strategy}

Prostatitis AND (Pelvic Pain OR CPP OR CP CPPS)

\section{Appendix 7. Abstract Proceedings}

\begin{tabular}{|c|c|}
\hline Conference & Website (last access October 2017) \\
\hline American Urology Association May 2017 & $\begin{array}{l}\text { www.jurology.com/is- } \\
\text { sue/S0022-5347(17)X0003-7 }\end{array}$ \\
\hline American Urology Association May 2016 & $\begin{array}{l}\text { www.jurology.com/is- } \\
\text { sue/S0022-5347(16)X0004-3 }\end{array}$ \\
\hline American Urology Association May 2015 & $\begin{array}{l}\text { www.jurology.com/is- } \\
\text { sue/S0022-5347(14)X0014-5 }\end{array}$ \\
\hline European Association of Urology 2017 & eau17.uroweb.org/ \\
\hline European Association of Urology 2016 & eaumunich2016.uroweb.org/resource-centre/ \\
\hline European Association of Urology 2015 & Not accessible \\
\hline Society of Sexual Medicine of North America 2017 annual meeting & www.smsna.org/V1/ \\
\hline \multicolumn{2}{|l|}{ Society of Sexual Medicine of North America 2016 annual meeting } \\
\hline Society of Sexual Medicine of North America 2015 annual meeting & \\
\hline
\end{tabular}

\section{WHAT'S NEW}

\begin{tabular}{lll}
\hline Date & Event & Description \\
\hline 4 April 2018 & $\begin{array}{l}\text { New citation required but conclusions } \\
\text { have not changed }\end{array}$ & $\begin{array}{l}\text { This version has received feedback from the Central Editorial } \\
\text { Unit regarding issues on GRADE judgements of Summary of Find- } \\
\text { ings table 1, abstract and plain language structure and language. } \\
\text { The authors have amended the review accordingly. }\end{array}$ \\
\hline A April 2018 & Amended & $\begin{array}{l}\text { This version has received feedback from the Central Editorial } \\
\text { Unit regarding issues on GRADE judgements of Summary of Find- } \\
\text { ings table 1, abstract and plain language structure and language. } \\
\text { The authors have amended the review accordingly. }\end{array}$ \\
&
\end{tabular}

\section{CONTRIBUTIONS OF AUTHORS}

JVAF: wrote the protocol, co-ordinated and performed all the tasks related to the review, and wrote the full report of the review.

TT: contributed to the methods section of the protocol, selected studies, extracted data and assessed risk of bias.

$\mathrm{JHJ}$ : retrieved studies, translated studies in Korean, selected studies, extracted data, assessed risk of bias, performed the GRADE assessments and provided clinical input in the conclusions. 
YX: retrieved studies, translated studies in Chinese, selected studies, extracted data and assessed risk of bias.

SI: retrieved studies, translated studies in Russian, assessed eligibility, selected studies, extracted data and assessed risk of bias.

VG: designed the search strategies and wrote the Search methods for identification of studies section, ran the searches and retrieved the included studies.

V: contributed to the methods section of the protocol, selected studies, extracted data and assessed risk of bias.

\section{DECLARATIONS OF INTEREST}

JVAF: none known.

TT: none known.

JHJ: none known.

YX: none known.

SI: none known.

VG: none known.

VV: none known.

\section{SOURCES OF SUPPORT}

\section{Internal sources}

- Instituto Universitario Hospital Italiano (IUHI), Argentina.

JVAF is the Cochrane Center Coordinator and VG is the Head Librarian; they both receive a salary from the IUHI.

\section{External sources}

- No sources of support supplied

\section{DIFFERENCES BETWEEN PROTOCOLANDREVIEW}

\section{Authors}

Federico Tirapegui, who was an author of the protocol, while contributing to some of the data extraction, was not an author of this review. We incorporated three additional authors who helped in the process of developing and writing this review.

\section{Background and methods: interventions}

We added more information about the included interventions in the 'Description of the intervention,' 'How the intervention might work' (Background) and the 'Type of interventions' (Methods). We deleted 'Clinical phenotyping' as an intervention in the methods section.

\section{Outcomes}

For clarity and a better understanding of the review and 'Summary of findings' tables, we renamed the 'Effectiveness of treatment' outcome to 'Prostatitis symptoms' and we specified the threshold for minimally clinical important differences for each outcome. This did not affect the assessment of the outcomes of the included studies since we have not changed their definition, but provided more clarity and transparency to the assessments. We included the response rate, defined as the number of participants achieving a minimal clinically important difference.

\section{Data collection and analysis}

We added two additional tables to summarise the 'Characteristics of the included studies' regarding participants and interventions.

We deleted the methods section regarding time-to-event data, since this type of information was not summarised in our review.

Due to the scarcity of data, we could not perform subgroup or sensitivity analysis except in the selected cases specified in the review.

As suggested by a peer reviewer, we added a sensitivity analysis taking into consideration the changes in the diagnostic criteria for CP/CPPS. 


\section{GRADE methods}

Due to the multiplicity of comparisons, we selected those comparisons with more than two RCTs for the drafting of the 'Summary of findings' table. Additionally, we constructed a 'Summary of findings' table for the following interventions: circumcision, physical activity and lifestyle modifications. Nonetheless, all other comparisons were rated using GRADE methodology.

We included an optimal information size (OIS) definition based on the predefined criteria for minimal clinically important difference (MCID) for 'Prostatitis symptoms' measured by NIH-CPSI score for the assessment of imprecision. For dichotomous outcomes and other continuous outcomes, we assessed the number of events and confidence intervals comparing them to the MCIDs.

We added a reference on how we translated the findings from GRADE to the plain language summary.

\section{NOTES}

We have based parts of the Methods section and Appendix 2 of this protocol on a standard template developed by the Cochrane Metabolic and Endocrine Disorders Group, which has been modified and adapted for use by the Cochrane Urology Group.

\section{N D EX TERMS}

\section{Medical Subject Headings (MeSH)}

Acupuncture Therapy [adverse effects] [methods]; Chronic Disease; Chronic Pain [ ${ }^{*}$ therapy]; Circumcision, Male [adverse effects]; Electromagnetic Radiation; Exercise; Extracorporeal Shockwave Therapy [adverse effects] [methods]; Hyperthermia, Induced [adverse effects] [methods]; Life Style; Massage [methods]; Pelvic Pain [ ${ }^{\star}$ therapy]; Prostatitis [ ${ }^{\star}$ therapy]; Randomized Controlled Trials as Topic

\section{MeSH check words}

Adult; Humans; Male 